\title{
Design and Methods of the California Stream Quality Assessment (CSQA), 2017
}

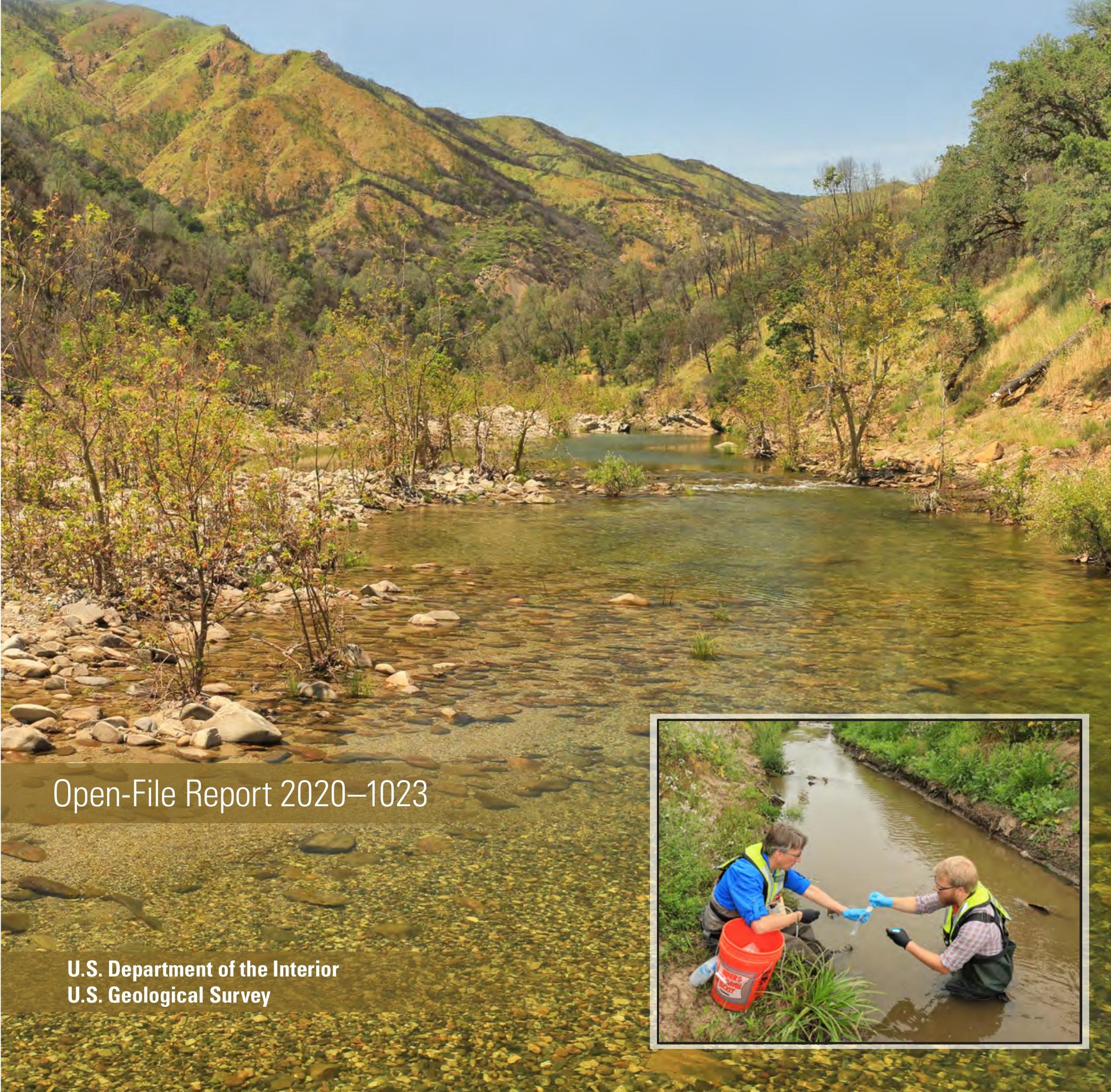


Front cover: Nacimiento River, California.

Inset. eDNA sampling by U.S. Geological Survey personnel in Alisal Creek, California. Photographs by Alan Cressler, U.S. Geological Survey.

Back Cover: Top left. Map of Central California Coastal study area and sampling sites.

Top right. Arroyo Seco at Elm Avenue Bridge, California. Photograph by Alan Cressler, U.S. Geological Survey.

Bottom left. Sediment sampling in Reclamation Ditch, California. Photograph by Alan Cressler, U.S. Geological Survey.

Bottom middle: Ceramic tile deployed to collect biofilms for pesticide analysis. Photograph by Barbara Mahler, U.S. Geological Survey.

Bottom right. Algae and invertebrate sampling in Alisal Creek, California. Photograph by Alan Cressler, U.S. Geological Survey. 


\section{Design and Methods of the California Stream Quality Assessment (CSOA), 2017}

By Jason T. May, Lisa H. Nowell, James F. Coles, Daniel T. Button, Amanda H. Bell, Sharon L. Qi, and Peter C. Van Metre

National Water Quality Program

Open-File Report 2020-1023 


\title{
U.S. Department of the Interior \\ DAVID BERNHARDT, Secretary
}

\author{
U.S. Geological Survey \\ James F. Reilly II, Director
}

U.S. Geological Survey, Reston, Virginia: 2020

For more information on the USGS - the Federal source for science about the Earth, its natural and living resources, natural hazards, and the environment—visit https://www.usgs.gov or call 1-888-ASK-USGS.

For an overview of USGS information products, including maps, imagery, and publications, visit https://store.usgs.gov/.

Any use of trade, firm, or product names is for descriptive purposes only and does not imply endorsement by the U.S. Government.

Although this information product, for the most part, is in the public domain, it also may contain copyrighted materials as noted in the text. Permission to reproduce copyrighted items must be secured from the copyright owner.

Suggested citation:

May, J.T., Nowell, L.H., Coles, J.F., Button, D.T., Bell, A.H., Qi, S.L., and Van Metre, P.C., 2020, Design and methods of the California stream quality assessment, 2017: U.S. Geological Survey Open-File Report 2020-1023, 88 p., https://doi.org/10.3133/ofr20201023.

ISSN 2331-1258 (online) 
Acknowledgment pages: Left page: Salinas River, California. Photograph by Alan Cressler, U.S. Geological Survey.

Right page: Jason May and Daniel Calhoun sampling for fish in Nanticoke Creek, NY, as part of the Northeast Stream Quality Assessment study. Photograph by Alan Cressler, U.S. Geological Survey. 


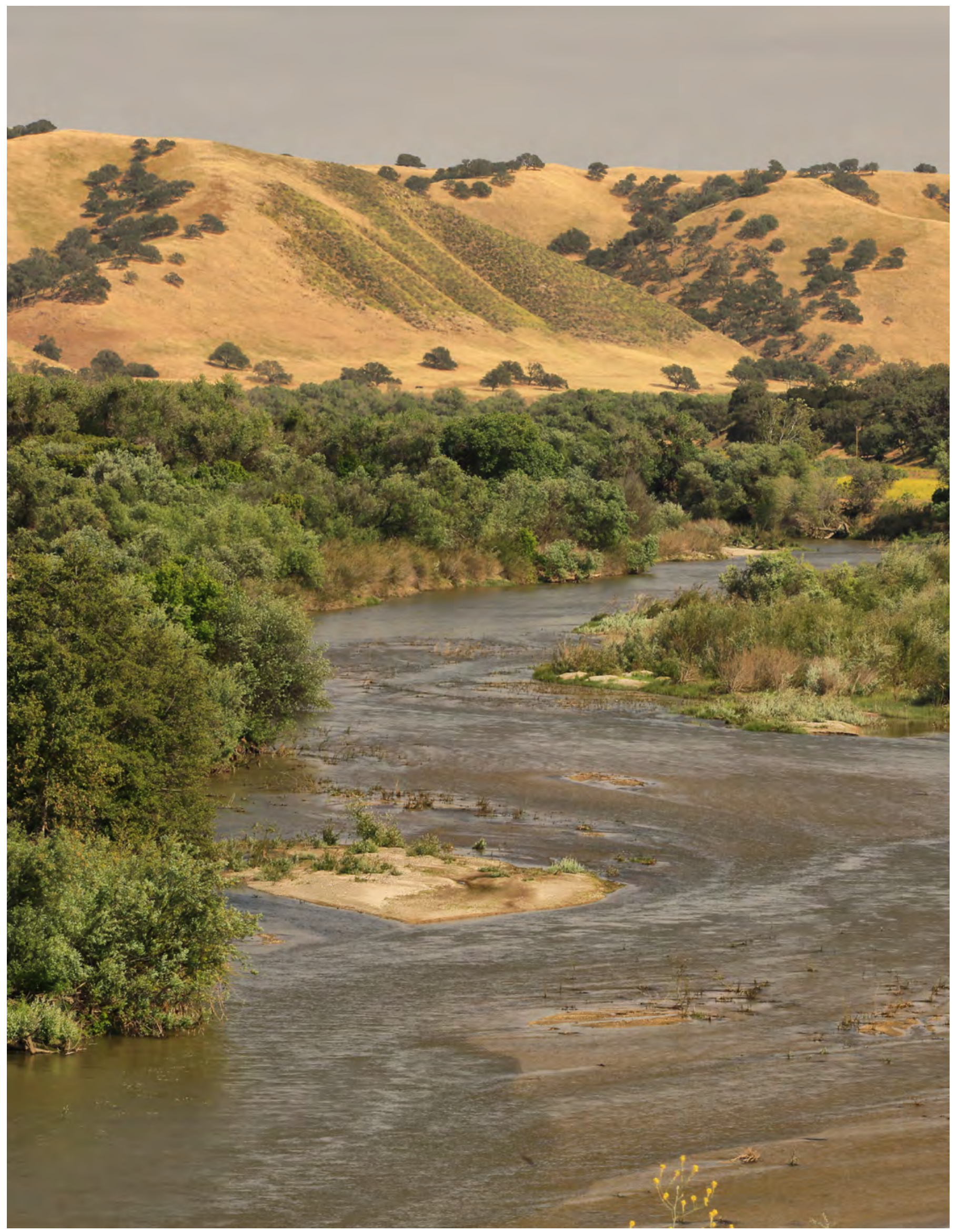




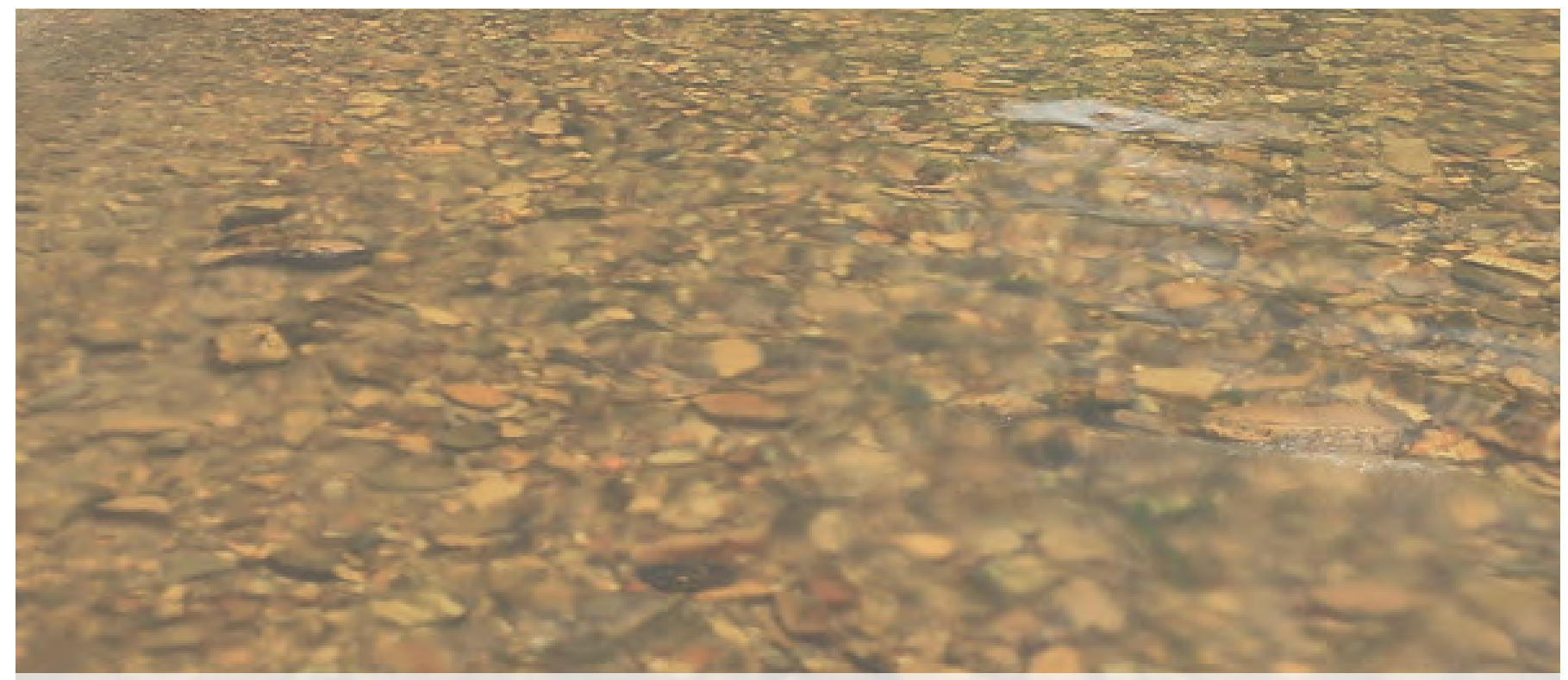

\section{Acknowledgments}

It is with deep appreciation and affection that we acknowledge the contributions of Jason Todd May to the planning and success of the California Stream Quality Assessment study. Sadly, Jason passed away on August 22, 2019, after a year-long illness. His efforts in study-site selection, organization of sampling crews, and data collection were invaluable in making the study a success. Jason's efforts on this study are a reflection of his dedication as a scientist at the California Water Science Center, where his broad experience was highly valued and resulted in his co-authoring more than 45 journal articles and U.S. Geological Survey reports. Jason was always willing to share his expertise with others inside and outside of the U.S. Geological Survey. His participation in the California Stream Quality Assessment and his dedication to quality science at the California Water Science Center will be missed.

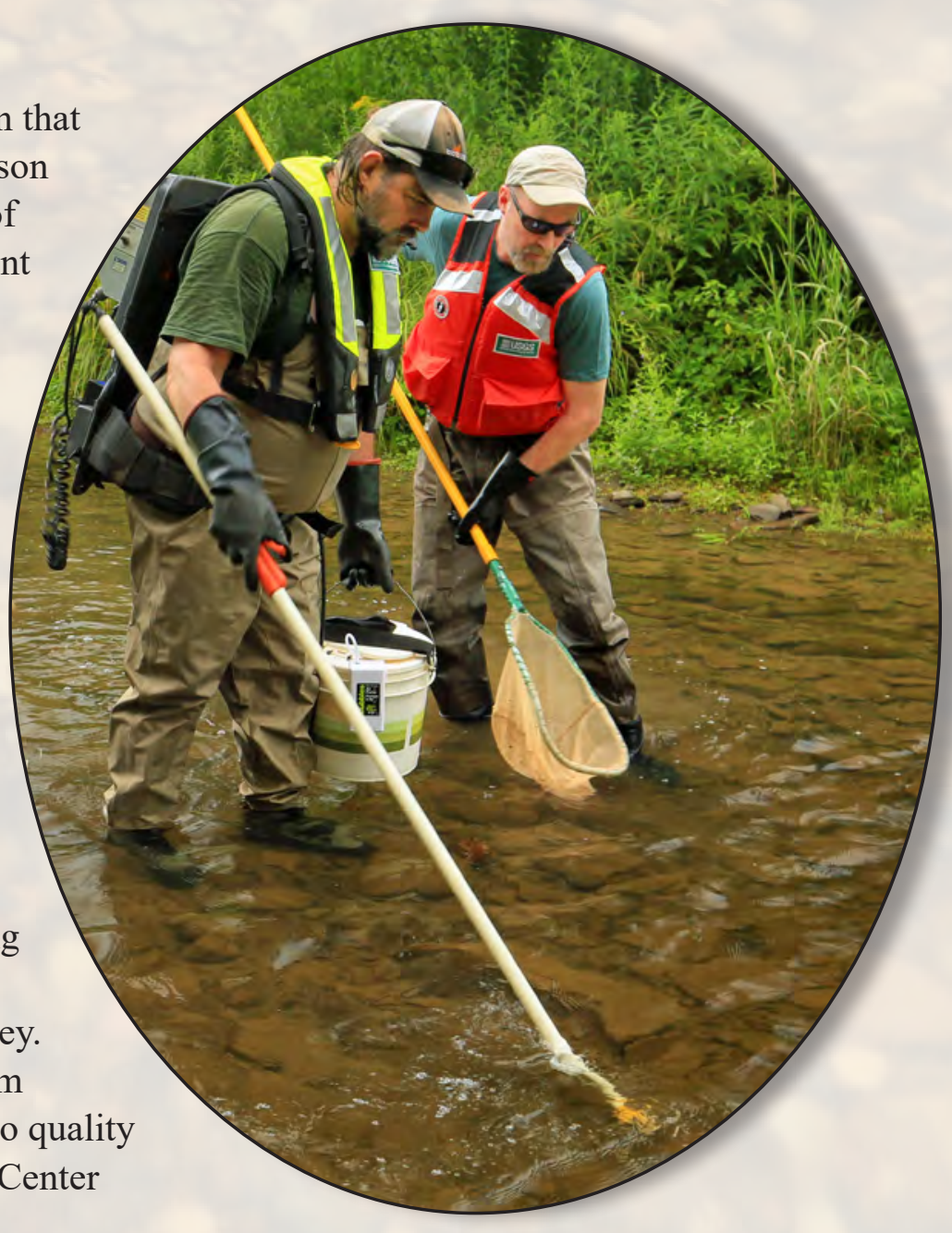




\section{Contents}

Abstract

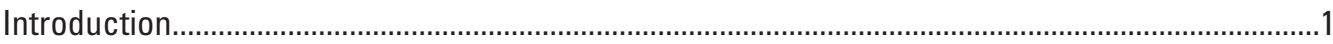

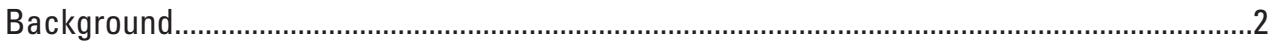

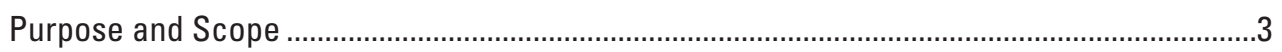

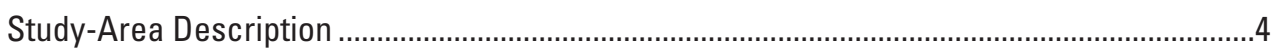

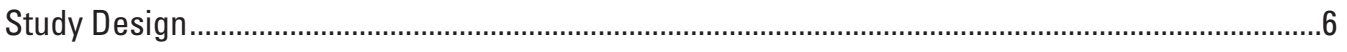

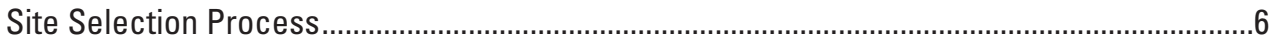

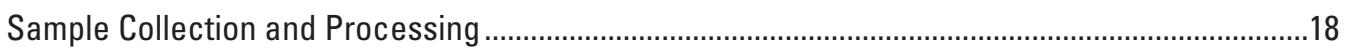

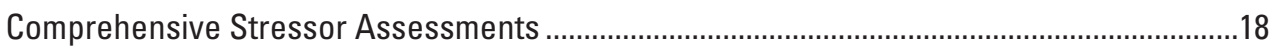

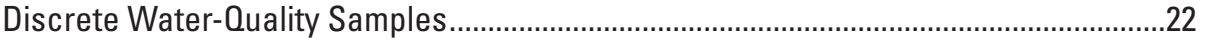

Polar Organic Compound Integrative Samplers (POCIS) _.................................................23

Continuous Streamflow, Stage, and Water Temperature ……………….........................23

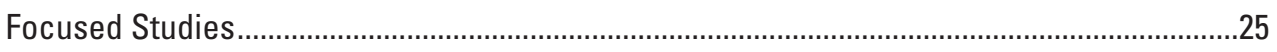

Sampling Pesticides with Small-Volume Pesticide Automated Samplers.......................25

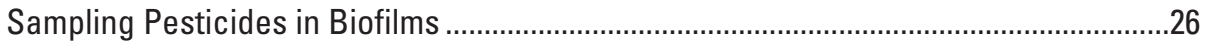

Sampling Suspended Sediment with Passive Samplers................................................26

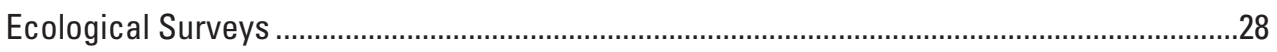

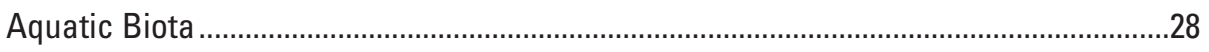

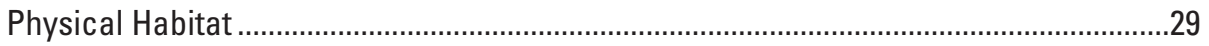

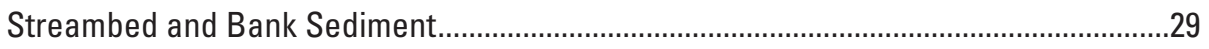

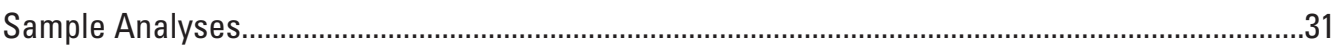

Comprehensive Stressor Assessments ..........................................................................

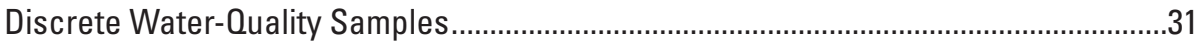

Polar Organic Compound Integrative Samplers ............................................................32

Continuous Streamflow, Stage, and Water Temperature ………………………...........32

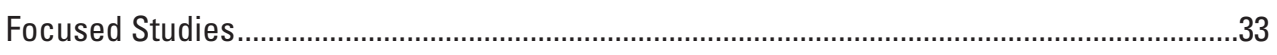

Pesticides from the Small-Volume Pesticide Automated Samplers ................................33

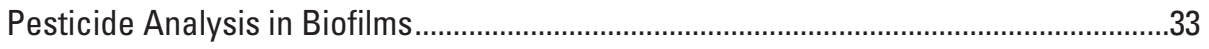

Suspended-Sediment Passive Samplers.....................................................................33

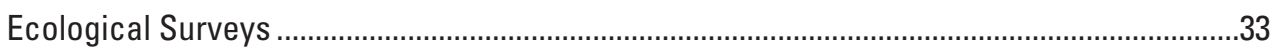

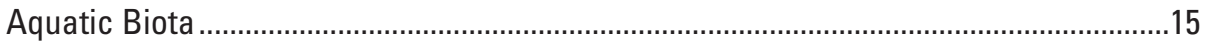

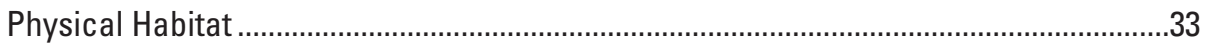

Streambed and Bank Sediment.................................................................................

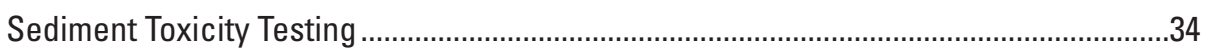

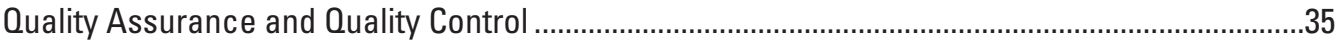

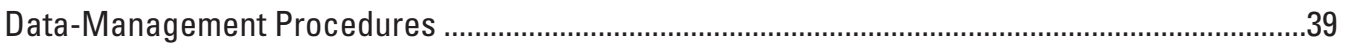

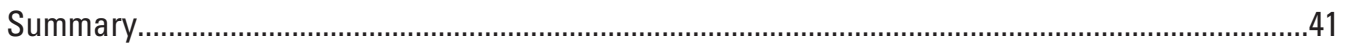

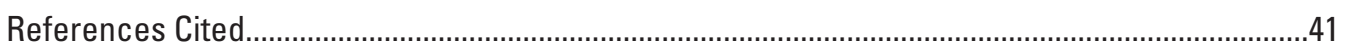

Appendix 1 Description of the Sampling Timelines, Matrix, Collection, and Processing for Water, Sediment, and Ecological Samples .................................................................48

Appendix 2 Description of the U.S. Geological Survey National Water Quality Laboratory Schedules Used for Water, Bed Sediment, and Biofilms ...................................57

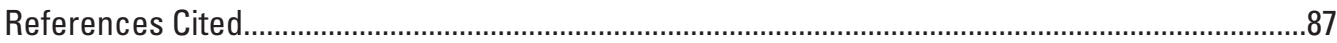




\section{Figures}

1. Map showing locations of the Regional Stream Quality Assessment studies across the United States.....

2. Map of the California Stream Quality Assessment study area, including sampling locations and generalized land cover.

3. Map showing locations of the 85 sites sampled for the California Stream Quality Assessment study, 2017

4. Graphs showing distribution of 85 California Stream Quality Assessment sites in relation to percent of cropland and percent of total urban for the whole basin and lower basin watershed delineation approaches

5. Photograph showing a hydrologist moving an isokinetic sampler into position..............23

6. Photograph showing deployed polar organic compound integrated sampler on cinder-block-and-cable infrastructure

7. Photographs showing the small-volume pesticide autosampler used to collect filtered water at sub-daily intervals for pesticide analysis at selected sites.

8. Photograph showing installation of time-integrating suspended-sediment passive samplers by U.S. Geological Survey personnel.

9. Photograph showing invertebrate sampling with a $D$-frame dip net by U.S. Geological Survey personnel .

10. Photograph showing collection of composite bed-sediment samples from a depositional area along a reach

\section{Tables}

1. Characteristics of stream watersheds in the Central California Foothills and Coastal Mountains ecoregion of the United States that were assessed as part of the U.S. Geological Survey National Water-Quality Assessment Project, California Stream Quality Assessment, in 2017

2. Land-use classes from the National Water Quality Assessment wall-to-wall anthropogenic land use trends database were summed to create land-use types .......14

3. Thresholds and combinations of land-use types used to assign sites to unique land-use categories

4. Distribution of 85 California Stream Quality Assessment sites by land-use category for the whole basin and lower basin watershed delineations

5. Summary of data collected at the California Stream Quality Assessment sites in 2017.

6. Summary counts of environmental, field blank, replicate, and spike samples of stream water from the 85 stream sites sampled in the California Stream Quality Assessment of the U.S. Geological Survey National Water-Quality Assessment Project in 2017

7. Summary counts of environmental, field replicate, field spike, and temporal samples of bed sediment collected from 82 stream sites in the California Stream Quality Assessment of the U.S. Geological Survey National Water-Quality Assessment Project in 2017 


\section{Conversion Factors}

International System of Units to U.S. customary units

\begin{tabular}{|c|c|c|}
\hline Multiply & By & To obtain \\
\hline \multicolumn{3}{|c|}{ Length } \\
\hline centimeter $(\mathrm{cm})$ & 0.3937 & inch (in.) \\
\hline millimeter $(\mathrm{mm})$ & 0.03937 & inch (in.) \\
\hline micrometer $(\mu \mathrm{m})$ & $3.937 \times 10^{-5}$ & inch (in.) \\
\hline nanometer (nm) & $3.937 \times 10^{-8}$ & inch (in.) \\
\hline meter $(\mathrm{m})$ & 3.281 & foot $(\mathrm{ft})$ \\
\hline kilometer $(\mathrm{km})$ & 0.6214 & mile (mi) \\
\hline meter $(\mathrm{m})$ & 1.094 & yard (yd) \\
\hline \multicolumn{3}{|c|}{ Area } \\
\hline square meter $\left(\mathrm{m}^{2}\right)$ & 0.0002471 & acre \\
\hline square meter $\left(\mathrm{m}^{2}\right)$ & 10.76 & square foot $\left(\mathrm{ft}^{2}\right)$ \\
\hline square centimeter $\left(\mathrm{cm}^{2}\right)$ & 0.1550 & square inch $\left(\mathrm{ft}^{2}\right)$ \\
\hline square kilometer $\left(\mathrm{km}^{2}\right)$ & 0.3861 & square mile $\left(\mathrm{mi}^{2}\right)$ \\
\hline \multicolumn{3}{|c|}{ Volume } \\
\hline milliliter $(\mathrm{mL})$ & 0.0002642 & gallon (gal) \\
\hline liter $(\mathrm{L})$ & 0.2642 & gallon (gal) \\
\hline liter $(\mathrm{L})$ & 61.02 & cubic inch (in $\left.{ }^{3}\right)$ \\
\hline \multicolumn{3}{|c|}{ Flow rate } \\
\hline meter per second $(\mathrm{m} / \mathrm{s})$ & 3.281 & foot per second $(\mathrm{ft} / \mathrm{s})$ \\
\hline \multicolumn{3}{|c|}{ Mass } \\
\hline gram $(\mathrm{g})$ & 0.03527 & ounce, avoirdupois (oz) \\
\hline nanogram (ng) & $3.527 \times 10^{-11}$ & ounce, avoirdupois (oz) \\
\hline gram per square meter $\left(\mathrm{g} / \mathrm{m}^{2}\right)$ & 0.0002048 & pound per square foot $\left(\mathrm{lb} / \mathrm{ft}^{2}\right)$ \\
\hline milligram per square meter $\left(\mathrm{mg} / \mathrm{m}^{2}\right)$ & 0.000003277 & ounce, avoirdupois, per square foot $\left(\mathrm{oz} / \mathrm{ft}^{2}\right)$ \\
\hline \multicolumn{3}{|c|}{ Pressure } \\
\hline kilopascal (kPa) & 0.1450 & pound-force per inch (lbf/in [or psi]) \\
\hline
\end{tabular}

Temperature in degrees Celsius $\left({ }^{\circ} \mathrm{C}\right)$ may be converted to degrees Fahrenheit $\left({ }^{\circ} \mathrm{F}\right)$ as follows: ${ }^{\circ} \mathrm{F}=\left(1.8 \times{ }^{\circ} \mathrm{C}\right)+32$.

Temperature in degrees Fahrenheit $\left({ }^{\circ} \mathrm{F}\right)$ may be converted to degrees Celsius $\left({ }^{\circ} \mathrm{C}\right)$ as follows: ${ }^{\circ} \mathrm{C}=\left({ }^{\circ} \mathrm{F}-32\right) / 1.8$. 


\title{
Datum
}

Horizontal coordinate information is referenced to the North American Datum of 1983 (NAD 83).

\section{Supplemental Information}

Specific conductance is given in microsiemens per centimeter at 25 degrees Celsius $(\mu \mathrm{S} / \mathrm{cm}$ at $\left.25^{\circ} \mathrm{C}\right)$.

Concentrations of chemical constituents in water are given either in milligrams per liter $(\mathrm{mg} / \mathrm{L})$, micrograms per liter $(\mu \mathrm{g} / \mathrm{L})$, or nanograms per liter $(\mathrm{ng} / \mathrm{L})$. Concentrations of chemical constituents in bed sediment are given in micrograms per kilogram $(\mu \mathrm{g} / \mathrm{kg})$.

\section{Abbreviations}

\author{
ASE accelerated solvent extraction \\ ASR analytical service request \\ CERC Columbia Environmental Research Center \\ CSQA California Stream Quality Assessment \\ DAI direct aqueous injection \\ DOC dissolved organic carbon \\ eDNA environmental DNA \\ ELISA enzyme-linked immunosorbent assay \\ EPA U.S. Environmental Protection Agency \\ ESI electrospray ionization \\ GC/MS gas chromatography mass spectrometry \\ GC-MS/MS gas chromatography-tandem mass spectrometry \\ GIS geographic information system \\ INSTAAR Institute of Arctic and Alpine Research \\ LC-MS/MS liquid chromatography tandem mass spectrometry
}




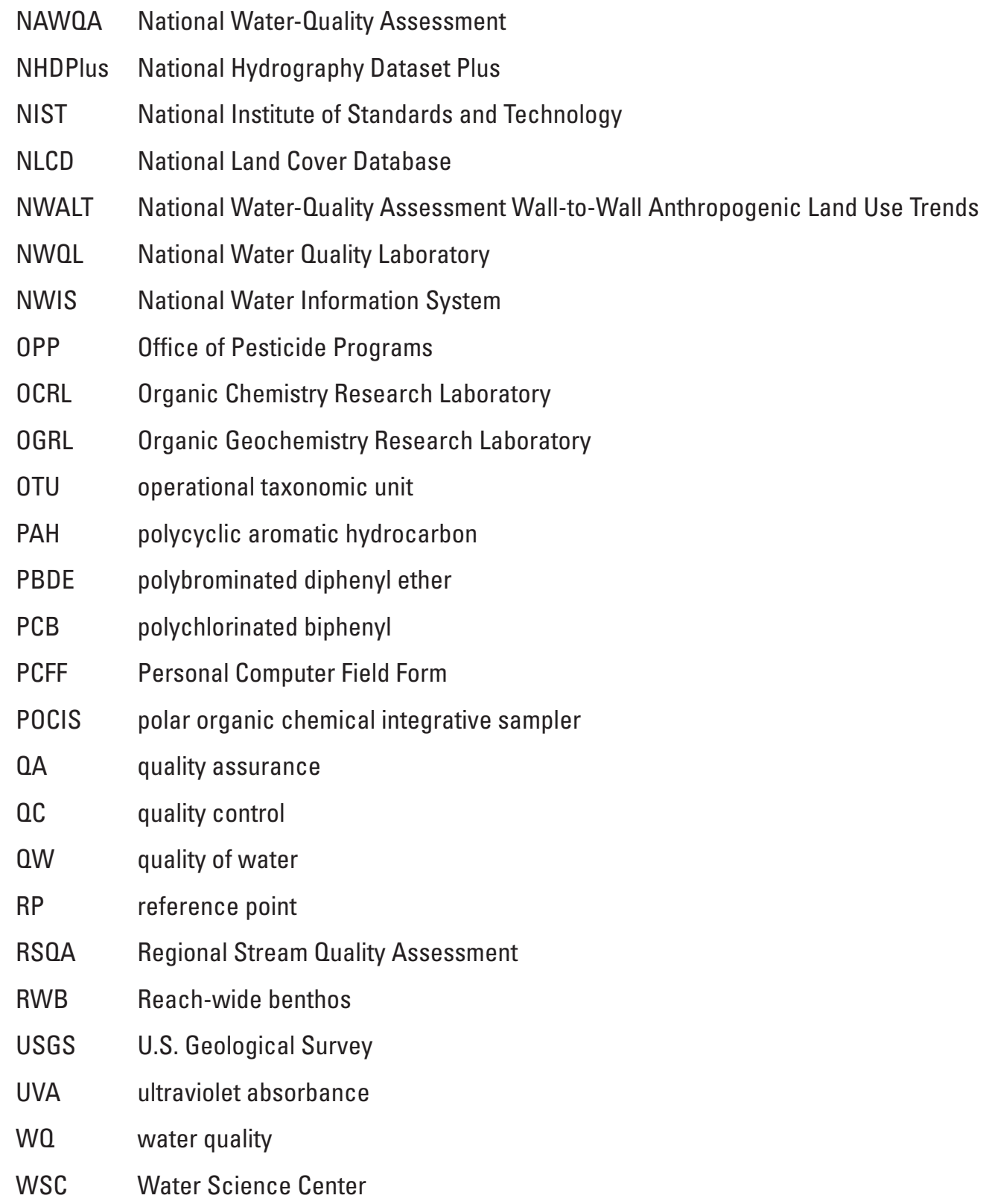




\title{
Design and Methods of the California Stream Quality Assessment (CSOA), 2017
}

\author{
By Jason T. May†, Lisa H. Nowell, James F. Coles, Daniel T. Button, Amanda H. Bell, Sharon L. Qi, and \\ Peter C. Van Metre
}

\section{Abstract}

During 2017, as part of the National Water-Quality Assessment Project, the U.S. Geological Survey conducted the California Stream Quality Assessment to investigate the quality of streams in the Central California Foothills and Coastal Mountains ecoregion, United States. The goal of the California Stream Quality Assessment study was to assess the health of wadeable streams in the region by characterizing multiple water-quality factors that are stressors to aquatic biota and by evaluating the relation between these stressors and biological indicators of stream health. Urbanization, agriculture, and modifications to streamflow are anthropogenic changes that affect water quality in the region; consequently, the study design primarily targeted sites and specific stressors associated with these activities. For the study, 85 stream sites were selected to represent the types and intensity of land use in the watershed; categories of site types were undeveloped, urban (low, medium, high), agriculture (low, high), and mixed (urban and agriculture). Most sites (about 70 percent) represent a gradient of urbanization from undeveloped to 99-percent urbanized. At most of the sites, streamgages or pressure transducers were used to monitor stream discharge and stage, as well as temperature. Water-quality samples were collected routinely at all sites and were analyzed for major ions, organic contaminants, nutrients, and suspended sediment. Sampling frequency varied on the basis of site type and location. Discrete water samples were collected weekly and generally 6 times per site, except for 11 undeveloped sites that were sampled only 4 times (during the last 4 weeks). Water sampling began at sites in the southern part of the study on March 13, 2017, and at sites in the northern part of the study on April 3, 2017. Passive samplers were deployed at most sites for measurement of polar organic contaminants (pesticides and pharmaceuticals). In May 2017, coincident

$\doteqdot$ Deceased August 22, 2019. with completion of water-quality sampling, an ecological survey was conducted at each site to assess benthic algal and macroinvertebrate communities and instream habitat. During the ecological surveys, a single composite streambed-sediment sample was collected for chemical analysis and toxicity testing. In addition, a few focused studies were done at subsets of sites, namely, measuring pesticides using small-volume automated samplers, measuring pesticides in biofilms, and sampling suspended sediments using passive samplers. This report describes the various study components and methods of the California Stream Quality Assessment, including measurements of water quality, sediment chemistry, habitat assessments, and ecological surveys, as well as procedures for sample analysis, quality assurance and quality control, and data management.

\section{Introduction}

Many natural and anthropogenic stressors can affect stream ecosystems, and often the stressors that degrade streams are associated with land use in the watershed (U.S. Environmental Protection Agency, 2013, 2019). Variations in streamflow, habitat, temperature, and levels of sediment and nutrients are essential characteristics of natural stream ecosystems, but deviation from the natural patterns of streams can substantially alter their biological condition and ecological function (Lenat and Crawford, 1994; Gregory and Calhoun, 2007; Nagy and others, 2011). Organic contaminants differ from other stressors in that many are derived from human activities and, through various modes of action and toxicity, are potentially detrimental to aquatic life as well as to humans who use water resources. To efficiently manage water resources, it is important to understand the conditions under which stressors - individually or in combinations - adversely affect the biological condition of streams and the water resources valued by people. 
Multistressor effects are often assessed in the laboratory, under controlled conditions, or in the field at small-catchment scales (Townsend and others, 2008; Culp and others, 2017). At these small scales, biogeochemical processes and complex environmental interactions can be manipulated and monitored; however, results of such studies are not readily extended over larger spatial scales. Alternatively, by characterizing the conditions of multiple streams over a large area, specific stressors and biological conditions can be evaluated on regional and national scales (U.S. Environmental Protection Agency, 2006; Herlihy and others, 2008); from such studies, empirical models have been developed to predict environmental stressors or the biological condition across national-scale disturbance gradients (Waite and others, 2000; Klemm and others, 2003; Herlihy and others, 2006; Coles and others, 2012). To date (2020), most regional- and national-scale studies have not included organic contaminants among the studied stressors (such as U.S. Environmental Protection Agency, 2016) or have generally limited their evaluations to relations between land use and biological condition (such as Gregory and Calhoun, 2007; Cuffney and others, 2011; Brown and others, 2012).

The U.S. Geological Survey (USGS), through the National Water-Quality Assessment (NAWQA) Project, is performing studies to bridge the gap in stressor coverage at large spatial scales, by extensively characterizing stressors (including contaminants) at the regional scale, with enough sampling sites to promote development of empirical models. As such, the studies are intended to provide the public and policymakers with information about the human and environmental factors that have the greatest effects on stream quality by addressing the following objectives in five regions of the United States:

1. Determine the status of stream quality across the region on the basis of contaminants, nutrients, sediments, toxicity of the bed sediments, streamflow, habitat, and biological communities;

2. Evaluate the relative influence of contaminants, nutrients, sediment, streamflow, and habitat on biological communities in the streams;

3. Identify how the natural and anthropogenic characteristics of the watersheds are related to stressors measured at the stream-reach scale and how the condition of biological communities can be explained by these stressors.

4. Develop statistical models and management tools to predict the ecological health of wadeable streams throughout the region and how ecological health is associated with concentrations of contaminants, nutrients, and sediment.

\section{Background}

The USGS launched Cycle III of the NAWQA Project in 2013, which marked the beginning of NAWQA's third decade of water-quality assessments for the Nation. In 1992, NAWQA began its Cycle I investigations, which aimed to characterize baseline water-quality conditions in the Nation's streams and aquifers (Leahy and others, 1990). A decade later, NAWQA transitioned to Cycle II, which emphasized trends and modeling and included five "topical" studies designed to improve our understanding of environmental processes affecting water quality. The topical studies addressed (1) the fate and transport of agricultural chemicals, (2) effects of urbanization on stream ecosystems, (3) effects of nutrient enrichment on stream ecosystems, (4) transport of contaminants to public-supply wells, and (5) bioaccumulation of mercury in stream ecosystems. Cycle III is built on 20 years of studies that describe contaminant sources and their transport to receiving waters, and the effects of land use on stream quality and ecological condition.

One of the major objectives in Cycle III is to assess the occurrence and effects of multiple instream stressors on stream ecological condition. Designated as Regional Stream Quality Assessment (RSQA) studies, these studies characterize watershed and stream water-quality stressors and aquatic biological conditions to improve understanding of stressor effects at regional scales (https://webapps.usgs.gov/RSQA/). Each RSQA study is a short-term assessment of wadeable streams in a targeted region, generally delineated by the U.S. Environmental Protection Agency (EPA) ecoregions (Omernik and Griffith, 2014). Between 75 and 100 streams are sampled in each RSQA study to investigate stream ecology and the effects of stressors that are primarily associated with urban development and agricultural land use. Wadeable streams are selected across gradients in urban or agricultural land use, depending on the dominant land use(s) in the region. Weekly water sampling typically is done for 4-12 weeks (depending on region and site characteristics) for a wide range of chemical constituents, and continuous monitoring of flow or stage and temperature is done at all stream sites. The timing of this water-quality (WQ) index period-defined as the 4- to 12-week period during which weekly discrete water samples were collected and analyzed for water-quality constituents - is designed to capture the spring and(or) early summer growing season, when pesticide and fertilizer applications are highest. Data from the WQ index period characterizes water-quality conditions antecedent to the ecological surveys, during which streambed sediment is collected for chemical analyses and toxicity testing, and stream habitat and biological communities are assessed. 
A RSQA study was conducted in the Central California Foothills and Coastal Mountains ecoregion (Omernik and Griffith, 2014) in 2017. Designated as the California Stream Quality Assessment (CSQA), this study included a network of 85 streams and associated watersheds, and was the last of five NAWQA Cycle III regional stream-quality assessment studies (fig. 1); other studies were the Midwest Stream Quality Assessment in 2013 (Garrett and others, 2017), the Southeast Stream Quality Assessment in 2014 (Journey and others, 2015), the Pacific Northwest Stream Quality Assessment in 2015 (Sheibley and others, 2017), and the Northeast Stream Quality Assessment in 2016 (Coles and others, 2019). Like the three most recent RSQA studies, the 2017 CSQA study was done to investigate stressors along an urban gradient because urbanization is particularly intense from San Francisco and Oakland to San Jose. Additional sites were selected to represent agriculture in the region, which includes vineyards, orchards, and various row crops, as well as mixed land uses.

\section{Purpose and Scope}

This report describes the design and methods of the CSQA study (Van Metre and others, 2017b). The methods described include the collection and processing of water- and sediment- quality samples, and surveys of stream habitat and algal and macroinvertebrate communities, at 85 stream sites. Methods also are described for several focused studies done at subsets of sites, and for the procedures of laboratory analysis, quality assurance (QA) and quality control (QC), and data management.

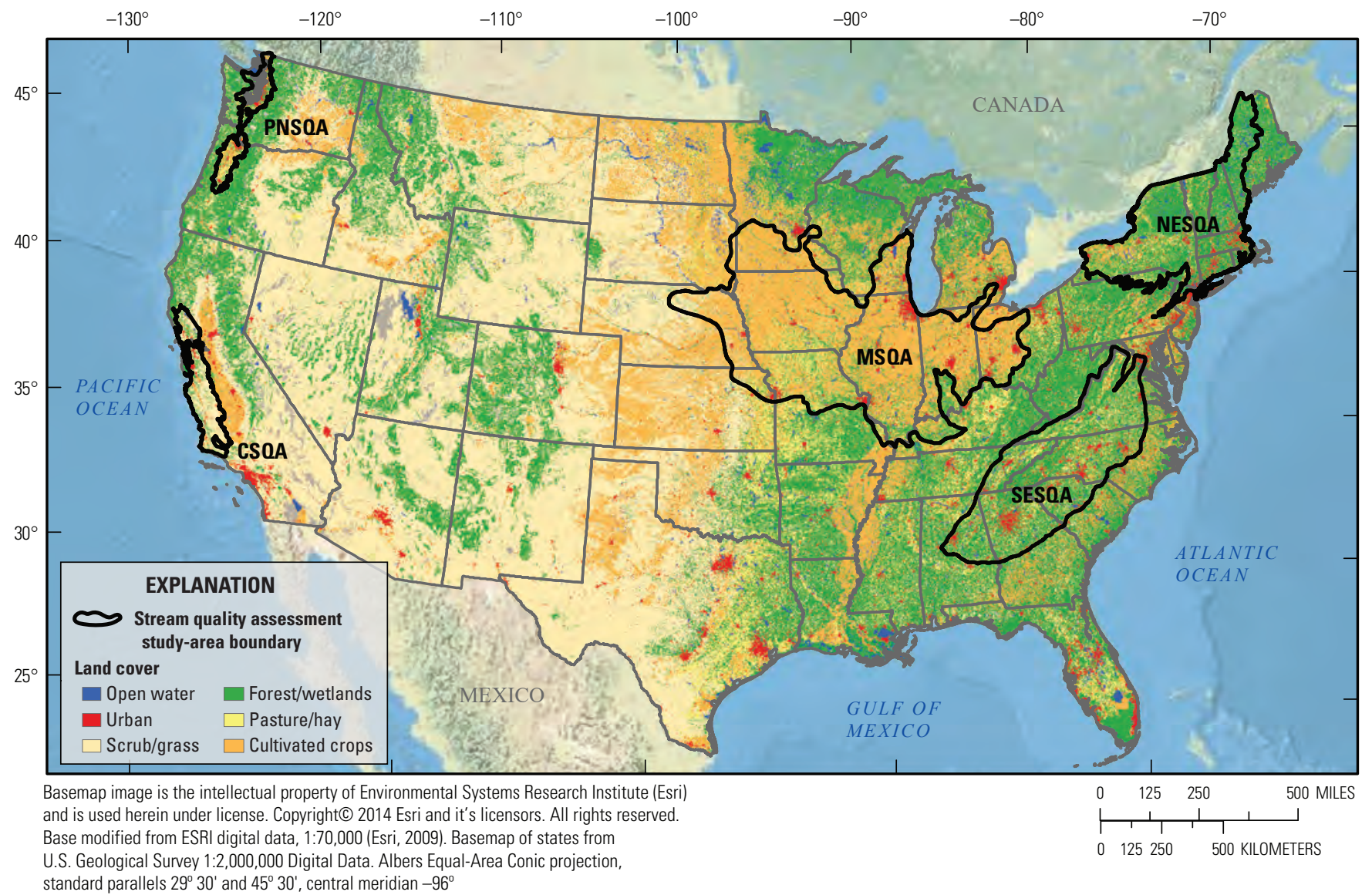

Figure 1. Locations of the Regional Stream Quality Assessment studies across the United States. (CSQA, California Stream Quality Assessment; MSQA, Midwest Stream Quality Assessment; NESQA, Northeast Stream Quality Assessment; PNSQA, Pacific Northwest Stream Quality Assessment; SESQA, Southeast Stream Quality Assessment). 


\section{Study-Area Description}

The 85 CSQA streams are distributed over much of the Central California Foothills and Coastal Mountains ecoregion, an EPA Level III ecoregion, where urbanization and agriculture in the larger valleys are associated with degradation of stream health (Ode and others, 2011). The following information describing the Central California Foothills and Coastal Mountains ecoregion (hereafter coastal California, modified from Griffith and others, 2016) is summarized from Wiken and others (2011) and Omernik and Griffith (2014).

A primary distinguishing characteristic of the coastal California ecoregion is its Mediterranean climate of hot dry summers and cool moist winters. The mean annual temperature ranges from approximately 14 degrees Celsius $\left({ }^{\circ} \mathrm{C}\right)$ to $18{ }^{\circ} \mathrm{C}$, and the frost-free period ranges from 180 to 365 days. The mean annual precipitation is 548 millimeters $(\mathrm{mm})$ and ranges from 200 to more than 1,400 $\mathrm{mm}$ on higher peaks in the northern portion of the ecoregion. Coastal fogs provide some moisture in the dry season. Vegetative cover is composed mainly of chaparral and oak woodlands, with grasslands occurring in some lower elevations and patches of pine at higher elevations. This ecoregion surrounds the lower and flatter Central California Valley ecoregion (although the CSQA sampled only on the western side of the Central Valley; fig. 2), and mostly consists of coastal terraces, some open low mountains or foothills, parallel ranges and valleys, and areas of irregular plains in the south and near the border of the Central California Valley ecoregion.

The geology of the coastal California ecoregion consists of Cenozoic marine and non-marine sedimentary rocks and Mesozoic granitic rocks; coarse sediments are found on colluvial slopes. Dominant soil orders include Alfisols, Entisols, and Mollisols, with a thermic soil temperature regime and xeric soil moisture regime. Streamflow in the region is mostly ephemeral and intermittent, with a few perennial streams entering from adjacent highland ecoregions. The region generally lacks lakes, but a few ponds and reservoirs occur. Large areas are in ranch lands grazed by domestic livestock, and some valleys are major agricultural centers, such as the Salinas Valley and the vineyards of Napa and Sonoma, but in general, little land has been cultivated. The highest levels of urban development are in the San Francisco Bay Area. 


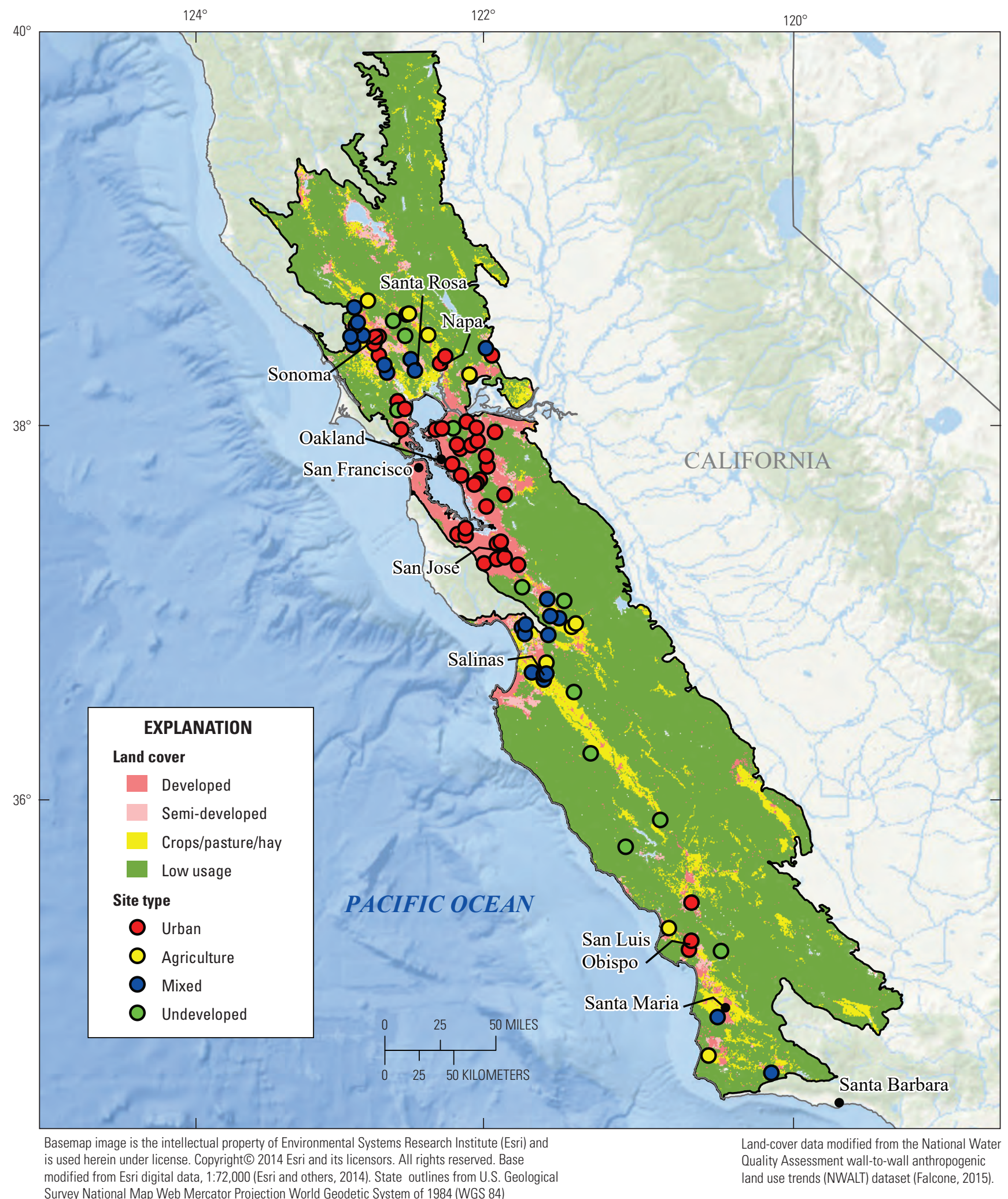

Figure 2. California Stream Quality Assessment study area, including sampling locations and generalized land cover. Site type indicates the dominant land use in the lower basin of the watershed. Land cover integrates land use from the National Water Quality Assessment wall-to-wall anthropogenic land use trends database (Falcone, 2015) and cropland from the Statewide Cropping Layer 2014 (Land IQ, LLC, 2017; California Department of Water Resources, 2019). 


\section{Study Design}

The CSQA study was designed to assess differences in stream quality associated with urban and agricultural development in the region and to identify and measure specific stressors potentially causing ecological impairment. To the extent possible, sites were selected to represent a full urban gradient (ranging from undeveloped to a high percentage of urban land use) as well as agricultural indicator basins (representing crops characteristic of the region and with minimal urban land). However, watersheds with mixtures of urban and agricultural uses are very common in coastal California, and the selection of some mixed land-use basins could not be avoided. The CSQA sites are located within a single Level III ecoregion to minimize natural variability in biological communities and allow for a better understanding of how anthropogenic factors might affect water quality and ecological condition.

The CSQA study followed the general study design for a RSQA study: sampling water- and sediment-quality at 75-100 wadeable streams across a region during a 4- to 12-week WQ index period (4-6 weeks in CSQA). Meanwhile, continuous streamflow (or stage) and water temperature data were collected, and the CSQA study culminated in an ecological survey of the sampling reach of the stream at the end of the WQ index period.

The three elements of the RSQA studies can be characterized as comprehensive stressor assessments, focused studies, and ecological surveys. Comprehensive stressor assessments were designed to be conducted at all sites, including assessment of basic water quality (such as major ions, nutrients, pesticides) in all discrete water samples, additional water-quality constituents (such as mercury, organic wastewater indicators, algal toxins) in discrete water samples at least once during the WQ index period, selected WQ constituents (pesticides and pharmaceuticals) that were accumulated (integrated) by passive samplers deployed during the WQ index period, and streamflow or stage and temperature data collected continuously throughout the WQ index period. Focused studies were conducted at a subset of sites to assess selected stressors in more detail; these consisted of pesticides in daily and weekly composite samples collected by autosamplers, pesticides in biofilms, and radionuclides and other constituents in suspended sediment collected by passive samplers. At the end of the WQ index period, the ecological surveys assessed physical habitat, surveyed aquatic biota communities, and sampled bed sediment for chemical analysis and toxicity testing. In the CSQA study, algal and macroinvertebrate communities were sampled during the ecological surveys. Unlike other RSQA studies, fish were not surveyed in CSQA because of concerns that electro-fishing in the CSQA streams might adversely affect sensitive species. Dates of sample collection for all elements of CSQA are shown by site in appendix table 1.1.

The CSQA presented some challenges that were unique relative to the previous RSQA studies: a longer pesticide application season, combined with variation in the timing of pesticide applications from south to north, and the potential for streams to go dry during late spring and early summer, especially in the south. Timing of the sampling activities to coincide with peak pesticide applications, especially for the more toxic insecticides and fungicides, was desirable to capture the highest potential exposure. Pesticide applications in coastal California generally occur earlier in the south than in the north, and the CSQA sampling regime was designed to accommodate this difference. Sampling from March through June over the entire study area would have been desirable from the perspective of pesticide exposure, but some small- to medium-sized streams in coastal California are intermittent, generally only flowing for a few months in late winter and spring. To improve the likelihood that continuous flow conditions would persist throughout the sampling period, an early sampling period was chosen, and the sites were divided into a "Southern" group $(n=36$; table 1) and a "Northern" group $(\mathrm{n}=49)$. The water sampling period was offset by 3 weeks between the Southern sites (March 13 to April 21, 2017; table 1) and the Northern sites (April 3 to May 12, 2017).

\section{Site Selection Process}

Candidate sites for the CSQA study were identified from active and historical (inactive) USGS streamgages $(n=125)$ and from sites monitored by the California Department of Fish and Wildlife $(n=435)$. A few candidate sites had streamgages nearby that were operated by the county (such as Contra Costa, Napa, San Luis Obispo, Solano) or city (Vacaville, Petaluma). Additional State and local agencies and institutions were consulted for information about candidate sites, including the California State Water Resources Control Board, North Coast Regional Water Quality Control Board, San Francisco Regional Water Quality Control Board, Central Coast Regional Water Quality Control Board, San Francisco Estuary Institute, California Department of Pesticide Regulation, and researchers from University of California at Davis and University of California at Berkeley. Several sites that had not been previously sampled were identified to fill gaps in the distribution of land-use settings relative to design objectives. A geospatial database was created that included land-cover characteristics for the watersheds of candidate sites (Qi and Nakagaki, 2020). 
Table 1. Characteristics of stream watersheds in the Central California Foothills and Coastal Mountains ecoregion of the United States that were assessed as part of the U.S. Geological Survey National Water-Quality Assessment Project, California Stream Quality Assessment, in 2017.

[Latitude and longitude of water quality sample location based on the North American Datum of 1983 and shown in decimal degrees. The column heading "Northern or Southern group" indicates relative location of sites in the San Francisco Bay area; sampling began the week of March 13, 2017, for the Southern group and April 3, 2017, for the Northern group. Sampling frequency is the number of discrete water samples collected at that site. Abbreviations: ID, identification; $\mathrm{km}^{2}$, square kilometer; NWIS, U.S. Geological Survey National Water Information System database; N, northern; S, southern]

\begin{tabular}{|c|c|c|c|c|c|c|c|c|c|c|c|}
\hline $\begin{array}{l}\text { Map ID } \\
\text { number1 }\end{array}$ & $\begin{array}{l}\text { NWIS station } \\
\text { number }\end{array}$ & NWIS station name & Field identifier & $\begin{array}{l}\text { Latitude } \\
\text { (NWIS) }\end{array}$ & $\begin{array}{l}\text { Longitude } \\
\text { (NWIS) }\end{array}$ & $\begin{array}{l}\text { Northern } \\
\text { or } \\
\text { Southern } \\
\text { group }\end{array}$ & $\begin{array}{l}\text { Basin } \\
\text { area } \\
\left(\mathrm{km}^{2}\right)\end{array}$ & $\begin{array}{l}\text { Whole basin } \\
\text { land use }\end{array}$ & $\begin{array}{l}\text { Lower basin } \\
\text { land use }{ }^{2}\end{array}$ & $\begin{array}{c}\text { Site type } \\
\text { (lower basin) }^{3}\end{array}$ & $\begin{array}{l}\text { Sampling } \\
\text { frequency }\end{array}$ \\
\hline 48 & 11179100 & ALAMEDA C NR FREMONT CA & CA_Alameda & 37.566602 & -122.001628 & $\mathrm{~N}$ & $1,654.4$ & Urban_low & Urban_med & Urban & 6 \\
\hline 18 & 382035121575501 & $\begin{array}{l}\text { ALAMO C A TULARE RD BR } \\
\text { NR VACAVILLE CA }\end{array}$ & CA_Alamo & 38.343083 & -121.96535 & $\mathrm{~N}$ & 55.9 & Urban_low & Urban_med & Urban & 6 \\
\hline 73 & 364003121373501 & $\begin{array}{l}\text { ALISAL C A FAIRVIEW AVE NR } \\
\text { SALINAS CA }\end{array}$ & CA_Alisal & 36.66725 & -121.626999 & S & 105.2 & Ag_high & Mixed & Mixed & 6 \\
\hline 47 & 11176900 & $\begin{array}{l}\text { ARROYO DE LA LAGUNA A } \\
\text { VERONA CA }\end{array}$ & $\begin{array}{l}\text { CA_Arroyo- } \\
\text { DeLaLa }\end{array}$ & 37.626599 & -121.883013 & $\mathrm{~S}$ & $1,045.6$ & Urban_low & Urban_med & Urban & 6 \\
\hline 27 & 380410122315501 & $\begin{array}{l}\text { ARROYO SAN JOSE A DIGITAL } \\
\text { DR NR NOVATO CA }\end{array}$ & CA_ArroyoDig & 38.069497 & -122.531997 & $\mathrm{~N}$ & 14.1 & Urban_med & Urban_med & Urban & 6 \\
\hline 29 & 11182400 & $\begin{array}{l}\text { ARROYO DEL HAMBRE A } \\
\text { MARTINEZ CA }\end{array}$ & CA_ArroyoMart & 38.003255 & -122.129965 & $\mathrm{~N}$ & 38.0 & Urban_low & Urban_low & Urban & 6 \\
\hline 28 & 380345122345201 & $\begin{array}{l}\text { ARROYO SAN JOSE A } \\
\text { FAIRWAY DR NR NOVATO CA }\end{array}$ & CA_ArroyoNov & 38.062272 & -122.581152 & $\mathrm{~N}$ & 2.3 & Undeveloped & Undeveloped & Undeveloped & 4 \\
\hline 75 & 11152000 & $\begin{array}{l}\text { ARROYO SECO NR SOLEDAD } \\
\text { CA }\end{array}$ & CA_ArroyoSeco & 36.280521 & -121.322706 & S & 625.4 & Undeveloped & Undeveloped & Undeveloped & 4 \\
\hline 78 & 352934120395501 & $\begin{array}{l}\text { ATASCADERO C A W MALL BR } \\
\text { A ATASCADERO CA }\end{array}$ & CA_Atascadero & 35.492883 & -120.665164 & S & 50.2 & Urban_low & Urban_med & Urban & 6 \\
\hline 46 & 11181008 & $\begin{array}{l}\text { CASTRO VALLEY C A } \\
\text { HAYWARD CA }\end{array}$ & CA_Castro & 37.679931 & -122.080519 & $\mathrm{~N}$ & 14.4 & Urban_high & Urban_high & Urban & 6 \\
\hline 79 & 352127120484501 & $\begin{array}{l}\text { CHORRO C A CHORRO C RD } \\
\text { NR MORRO BAY CA }\end{array}$ & CA_Chorro & 35.357508 & -120.812464 & S & 105.0 & Urban_low & Ag_low & Agriculture & 6 \\
\hline 74 & 363608121255201 & $\begin{array}{l}\text { CHUALAR C A CHUALAR } \\
\text { CANYON RD NR CHUALAR } \\
\text { CA }\end{array}$ & CA_ChuChuChu & 36.6022 & -121.430997 & S & 58.6 & Undeveloped & Undeveloped & Undeveloped & 4 \\
\hline 14 & 11465690 & $\begin{array}{l}\text { COLGAN C NR SANTA ROSA } \\
\text { CA }\end{array}$ & CA_Colgan & 38.402135 & -122.733043 & $\mathrm{~N}$ & 9.0 & Urban_high & Urban_high & Urban & 6 \\
\hline 8 & 11456500 & CONN C NR OAKVILLE CA & CA_ConnOak & 38.447278 & -122.380556 & $\mathrm{~N}$ & 146.1 & Ag_low & Ag_high & Agriculture & 6 \\
\hline 17 & 11465660 & $\begin{array}{l}\text { COPELAND C A ROHNERT } \\
\text { PARK CA }\end{array}$ & CA_Copeland & 38.343248 & -122.701932 & $\mathrm{~N}$ & 14.9 & Urban_med & Urban_high & Urban & 6 \\
\hline 66 & 11159200 & $\begin{array}{l}\text { CORRALITOS C A FREEDOM } \\
\text { CA }\end{array}$ & CA_Corralitos & 36.939397 & -121.770506 & S & 70.8 & Mixed & Mixed & Mixed & 6 \\
\hline 33 & 11460000 & CORTE MADERA C A ROSS CA & CA_Corte & 37.962979 & -122.556922 & $\mathrm{~N}$ & 46.7 & Urban_med & Urban_med & Urban & 6 \\
\hline
\end{tabular}


Table 1. Characteristics of stream watersheds in the Central California Foothills and Coastal Mountains ecoregion of the United States that were assessed as part of the U.S. Geological Survey National Water-Quality Assessment Project, California Stream Quality Assessment, in 2017._Continued

[Latitude and longitude of water quality sample location based on the North American Datum of 1983 and shown in decimal degrees. The column heading "Northern or Southern group" indicates relative location of sites in the San Francisco Bay area; sampling began the week of March 13, 2017, for the Southern group and April 3, 2017, for the Northern group. Sampling frequency is the number of discrete water samples collected at that site. Abbreviations: ID, identification; $\mathrm{km}^{2}$, square kilometer; NWIS, U.S. Geological Survey National Water Information System database; N, northern; S, southern]

\begin{tabular}{|c|c|c|c|c|c|c|c|c|c|c|c|}
\hline $\begin{array}{c}\text { Map iden- } \\
\text { tification } \\
\text { number1 }\end{array}$ & $\begin{array}{l}\text { NWIS station } \\
\text { number }\end{array}$ & NWIS station name & Field identifier & $\begin{array}{l}\text { Latitude } \\
\text { (NWIS) }\end{array}$ & $\begin{array}{l}\text { Longitude } \\
\text { (NWIS) }\end{array}$ & $\begin{array}{c}\text { Northern } \\
\text { or } \\
\text { Southern } \\
\text { group }\end{array}$ & $\begin{array}{l}\text { Basin } \\
\text { area } \\
\left(\mathrm{km}^{2}\right)\end{array}$ & $\begin{array}{l}\text { Whole basin } \\
\text { land use }\end{array}$ & $\begin{array}{l}\text { Lower basin } \\
\text { land use }^{2}\end{array}$ & $\begin{array}{c}\text { Site type } \\
\text { (lower basin) }\end{array}$ & $\begin{array}{l}\text { Sampling } \\
\text { frequency }\end{array}$ \\
\hline 52 & 372303121542901 & $\begin{array}{l}\text { COYOTE C BL CHARCOT AVE } \\
\text { NR SAN JOSE CA }\end{array}$ & CA_CoyoteChar & 37.384453 & -121.907849 & $\mathrm{~N}$ & 809.7 & Urban_low & Urban_high & Urban & 6 \\
\hline 57 & 371554121474101 & $\begin{array}{l}\text { COYOTE C A COYOTE RD NR } \\
\text { SAN JOSE CA }\end{array}$ & CA_CoyoteCoyote & 37.264939 & -121.794658 & S & 594.4 & Urban_low & Urban_med & Urban & 6 \\
\hline 60 & 11169800 & COYOTE C NR GILROY CA & CA_CoyoteGil & 37.077723 & -121.494383 & $\mathrm{~S}$ & 282.5 & Undeveloped & Undeveloped & Undeveloped & 4 \\
\hline 44 & 11180900 & CROW C NR HAYWARD CA & CA_Crow & 37.70493 & -122.043851 & $\mathrm{~N}$ & 27.3 & Urban_low & Urban_low & Urban & 6 \\
\hline 2 & 11465350 & $\begin{array}{l}\text { DRY C NR MOUTH NR } \\
\text { HEALDSBURG CA }\end{array}$ & CA_DryMouth & 38.58741 & -122.862216 & $\mathrm{~N}$ & 565.0 & Ag_low & Mixed & Mixed & 6 \\
\hline 72 & 364138121373701 & $\begin{array}{l}\text { GABILAN C AB E LAUREL DR } \\
\text { NR SALINAS CA }\end{array}$ & CA_GabilanEastL & 36.693963 & -121.627252 & $\mathrm{~S}$ & 107.7 & Ag_low & Mixed & Mixed & 6 \\
\hline 69 & 11152600 & GABILAN C NR SALINAS CA & CA_GabilanSal & 36.755792 & -121.610501 & S & 95.1 & Undeveloped & Ag_low & Agriculture & 6 \\
\hline 30 & 375819122035801 & $\begin{array}{l}\text { GRAYSON C A GOLF CLUB RD } \\
\text { NR PLEASANT HILL CA }\end{array}$ & CA_Grayson & 37.972091 & -122.066128 & $\mathrm{~N}$ & 36.5 & Urban_high & Urban_high & Urban & 6 \\
\hline 12 & 382619122531401 & $\begin{array}{l}\text { GREEN VALLEY C A GRATON } \\
\text { CA }\end{array}$ & CA_Green & 38.438729 & -122.887208 & $\mathrm{~N}$ & 25.7 & Mixed & Mixed & Mixed & 6 \\
\hline 53 & 11169025 & $\begin{array}{l}\text { GUADALUPE R ABV HWY } 101 \\
\text { A SAN JOSE CA }\end{array}$ & CA_GuadalupeA & 37.37383 & -121.933013 & $\mathrm{~S}$ & 406.4 & Urban_med & Urban_high & Urban & 6 \\
\hline 54 & 371814121525601 & $\begin{array}{l}\text { GUADALUPE R A WILLOW } \\
\text { GLEN WAY NR SAN JOSE CA }\end{array}$ & CA_GuadalupeB & 37.303947 & -121.88225 & $\mathrm{~S}$ & 219.0 & Urban_med & Urban_high & Urban & 6 \\
\hline 38 & 375257122050001 & $\begin{array}{l}\text { LAS TRAMPAS C A } \\
\text { LAFAYETTE CA }\end{array}$ & CA_LasTrampas & 37.882397 & -122.099997 & $\mathrm{~N}$ & 20.4 & Urban_low & Urban_med & Urban & 6 \\
\hline 22 & 381740122395901 & LICHAU C A PENNGROVE CA & CA_Lichau & 38.294481 & -122.666361 & $\mathrm{~N}$ & 20.0 & Mixed & Mixed & Mixed & 6 \\
\hline 62 & 11153650 & LLAGAS C NR GILROY & CA_LlagasGilroy & 36.987449 & -121.527162 & S & 218.9 & Mixed & Mixed & Mixed & 6 \\
\hline 59 & 370512121361901 & $\begin{array}{l}\text { LLAGAS C A SAN MARTIN } \\
\text { AVE A SAN MARTIN CA }\end{array}$ & CA_LlagasMartin & 37.086742 & -121.605206 & S & 73.0 & Urban_low & Mixed & Mixed & 6 \\
\hline 58 & 11153470 & $\begin{array}{l}\text { LLAGAS C AB CHESBRO RES } \\
\text { NR MORGAN HILL CA }\end{array}$ & CA_LlagasMorgan & 37.148333 & -121.768333 & S & 24.9 & Undeveloped & Undeveloped & Undeveloped & 4 \\
\hline 82 & 11141280 & $\begin{array}{l}\text { LOPEZ C NR ARROYO } \\
\text { GRANDE CA }\end{array}$ & CA_Lopez & 35.23553 & -120.472386 & $\mathrm{~S}$ & 54.1 & Undeveloped & Undeveloped & Undeveloped & 4 \\
\hline 55 & 371738121555901 & $\begin{array}{l}\text { LOS GATOS C A E HAMILTON } \\
\text { AVE NR CAMPBELL CA }\end{array}$ & CA_LosGatos & 37.2939 & -121.933 & S & 119.1 & Urban_low & Urban_high & Urban & 6 \\
\hline
\end{tabular}


Table 1. Characteristics of stream watersheds in the Central California Foothills and Coastal Mountains ecoregion of the United States that were assessed as part of the U.S. Geological Survey National Water-Quality Assessment Project, California Stream Quality Assessment, in 2017.—Continued

[Latitude and longitude of water quality sample location based on the North American Datum of 1983 and shown in decimal degrees. The column heading "Northern or Southern group" indicates relative location of sites in the San Francisco Bay area; sampling began the week of March 13, 2017, for the Southern group and April 3, 2017, for the Northern group. Sampling frequency is the number of discrete water samples collected at that site. Abbreviations: ID, identification; $\mathrm{km}^{2}$, square kilometer; NWIS, U.S. Geological Survey National Water Information System database; N, northern; S, southern]

\begin{tabular}{|c|c|c|c|c|c|c|c|c|c|c|c|}
\hline $\begin{array}{l}\text { Map iden- } \\
\text { tification } \\
\text { number1 }\end{array}$ & $\begin{array}{l}\text { NWIS station } \\
\text { number }\end{array}$ & NWIS station name & Field identifier & $\begin{array}{l}\text { Latitude } \\
\text { (NWIS) }\end{array}$ & $\begin{array}{l}\text { Longitude } \\
\text { (NWIS) }\end{array}$ & $\begin{array}{l}\text { Northern } \\
\text { or } \\
\text { Southern } \\
\text { group }\end{array}$ & $\begin{array}{l}\text { Basin } \\
\text { area } \\
\left(\mathrm{km}^{2}\right)\end{array}$ & $\begin{array}{l}\text { Whole basin } \\
\text { land use }\end{array}$ & $\begin{array}{l}\text { Lower basin } \\
\text { land use }{ }^{2}\end{array}$ & $\begin{array}{c}\text { Site type } \\
\text { (lower basin)3 }\end{array}$ & $\begin{array}{l}\text { Sampling } \\
\text { frequency }\end{array}$ \\
\hline 1 & 383719122462501 & $\begin{array}{l}\text { MAACAMA C A CHALK HILL } \\
\text { RD BR NR HEALDSBURG CA }\end{array}$ & CA_Maacama & 38.621958 & -122.773689 & $\mathrm{~N}$ & 118.0 & Ag_low & Ag_low & Agriculture & 6 \\
\hline 7 & 11466800 & $\begin{array}{l}\text { MARK WEST C NR MIRABEL } \\
\text { HEIGHTS CA }\end{array}$ & CA_MarkWMir & 38.494078 & -122.853326 & $\mathrm{~N}$ & 652.0 & Mixed & Mixed & Mixed & 6 \\
\hline 5 & 383109122363301 & $\begin{array}{l}\text { MARK WEST C A TARWATER } \\
\text { RD NR CALISTOGA CA }\end{array}$ & CA_MarkWTar & 38.519047 & -122.609222 & $\mathrm{~N}$ & 10.5 & Undeveloped & Undeveloped & Undeveloped & 4 \\
\hline 51 & 372500122081201 & $\begin{array}{l}\text { MATADERO C A JOSINA AVE A } \\
\text { PALO ALTO CA }\end{array}$ & CA_Matadero & 37.4168 & -122.136592 & S & 16.1 & Urban_med & Urban_high & Urban & 6 \\
\hline 11 & 11466170 & $\begin{array}{l}\text { MATANZAS C A SANTA ROSA } \\
\text { CA }\end{array}$ & CA_Matanzas & 38.438801 & -122.702487 & $\mathrm{~N}$ & 57.0 & Mixed & Urban_med & Urban & 6 \\
\hline 19 & 382017122161101 & $\begin{array}{l}\text { MILLIKEN C BL HEDGESIDE } \\
\text { AVE NR NAPA CA }\end{array}$ & CA_Milliken & 38.338155 & -122.269874 & $\mathrm{~N}$ & 45.0 & Urban_low & Urban_low & Urban & 6 \\
\hline 35 & 375701121564401 & MT DIABLO C A CLAYTON CA & CA_MtDiablo & 37.950364 & -121.945647 & $\mathrm{~N}$ & 42.7 & Urban_low & Urban_med & Urban & 6 \\
\hline 77 & 11148900 & $\begin{array}{l}\text { NACIMIENTO R BL SAPAQUE } \\
\text { C NR BRYSON CA }\end{array}$ & CA_NaciBryson & 35.788579 & -121.093805 & $\mathrm{~S}$ & 403.3 & Undeveloped & Undeveloped & Undeveloped & 4 \\
\hline 3 & 383321122302101 & $\begin{array}{l}\text { NAPA R A BALE LN NR DEER } \\
\text { PARK CA }\end{array}$ & CA_NapaBale & 38.555946 & -122.505915 & $\mathrm{~N}$ & 116.5 & Ag_low & Ag_low & Agriculture & 6 \\
\hline 21 & 11458300 & NAPA C A NAPA & CA_NapaNapa & 38.301859 & -122.303863 & $\mathrm{~N}$ & 38.9 & Urban_low & Urban_med & Urban & 6 \\
\hline 71 & 364155121363901 & NATIVIDAD C NR SALINAS CA & CA_Natividad & 36.6987 & -121.610722 & S & 25.9 & Ag_low & Mixed & Mixed & 6 \\
\hline 15 & 382346122521201 & $\begin{array}{l}\text { UNNAMED TRIB A } \\
\text { MONTGOMERY RD NR } \\
\text { SEBASTOPOL CA }\end{array}$ & $\begin{array}{l}\text { CA_NoNameMon- } \\
\text { ty }\end{array}$ & 38.396125 & -122.870039 & $\mathrm{~N}$ & 14.9 & Mixed & Mixed & Mixed & 6 \\
\hline 64 & 365718121444301 & $\begin{array}{l}\text { UNNAMED TRIB A PAULSEN } \\
\text { RD NR FREEDOM CA }\end{array}$ & CA_NoNamePaul & 36.955181 & -121.745631 & $\mathrm{~S}$ & 42.8 & Mixed & Mixed & Mixed & 6 \\
\hline 26 & 11459500 & NOVATO C A NOVATO CA & CA_Novato & 38.107698 & -122.579981 & $\mathrm{~N}$ & 46.5 & Urban_low & Urban_med & Urban & 6 \\
\hline 83 & 11141050 & ORCUTT C NR ORCUTT CA & CA_Orcutt & 34.88359 & -120.494888 & S & 49.3 & Urban_low & Mixed & Mixed & 6 \\
\hline 63 & 365736121250801 & $\begin{array}{l}\text { PACHECO C A SAN FELIPE RD } \\
\text { NR DUNNEVILLE CA }\end{array}$ & CA_Pacheco & 36.959818 & -121.418968 & S & 395.3 & Undeveloped & Ag_high & Agriculture & 6 \\
\hline 68 & 11159000 & PAJARO R A CHITTENDEN CA & CA_PajaroChit & 36.900231 & -121.597721 & $\mathrm{~S}$ & $3,071.5$ & Ag_low & Mixed & Mixed & 6 \\
\hline 67 & 11159500 & $\begin{array}{l}\text { PAJARO R A WATSONVILLE } \\
\text { CA }\end{array}$ & CA_PajaroWat & 36.905297 & -121.7514 & S & $3,284.6$ & Ag_low & Mixed & Mixed & 6 \\
\hline
\end{tabular}


Table 1. Characteristics of stream watersheds in the Central California Foothills and Coastal Mountains ecoregion of the United States that were assessed as part of the U.S. Geological Survey National Water-Quality Assessment Project, California Stream Quality Assessment, in 2017.-Continued

[Latitude and longitude of water quality sample location based on the North American Datum of 1983 and shown in decimal degrees. The column heading "Northern or Southern group" indicates relative location of sites in the San Francisco Bay area; sampling began the week of March 13, 2017, for the Southern group and April 3, 2017, for the Northern group. Sampling frequency is the number of discrete water samples collected at that site. Abbreviations: ID, identification; $\mathrm{km}^{2}$, square kilometer; NWIS, U.S. Geological Survey National Water Information System database; N, northern; S, southern]

\begin{tabular}{|c|c|c|c|c|c|c|c|c|c|c|c|}
\hline $\begin{array}{l}\text { Map iden- } \\
\text { tification } \\
\text { number }\end{array}$ & $\begin{array}{l}\text { NWIS station } \\
\text { number }\end{array}$ & NWIS station name & Field identifier & $\begin{array}{l}\text { Latitude } \\
\text { (NWIS) }\end{array}$ & $\begin{array}{l}\text { Longitude } \\
\text { (NWIS) }\end{array}$ & $\begin{array}{l}\text { Northern } \\
\text { or } \\
\text { Southern } \\
\text { group }\end{array}$ & $\begin{array}{l}\text { Basin } \\
\text { area } \\
\left(\mathrm{km}^{2}\right)\end{array}$ & $\begin{array}{l}\text { Whole basin } \\
\text { land use }\end{array}$ & $\begin{array}{l}\text { Lower basin } \\
\text { land use }{ }^{2}\end{array}$ & $\begin{array}{c}\text { Site type } \\
\text { (lower basin) }\end{array}$ & $\begin{array}{l}\text { Sampling } \\
\text { frequency }\end{array}$ \\
\hline 24 & 381519122385601 & $\begin{array}{l}\text { PETALUMA R NR PETALUMA } \\
\text { CA }\end{array}$ & CA_Petaluma & 38.255189 & -122.648971 & $\mathrm{~N}$ & 87.1 & Mixed & Mixed & Mixed & 6 \\
\hline 31 & 375807122124001 & $\begin{array}{l}\text { PINOLE C BL ALHAMBRA } \\
\text { VALLEY RD NR PINOLE CA }\end{array}$ & CA_Pinole & 37.968652 & -122.210997 & $\mathrm{~N}$ & 13.4 & Undeveloped & Undeveloped & Undeveloped & 4 \\
\hline 70 & 11152650 & $\begin{array}{l}\text { RECLAMATION DITCH NR } \\
\text { SALINAS CA }\end{array}$ & CA_Reclamation & 36.704959 & -121.704948 & $\mathrm{~S}$ & 274.6 & Mixed & Mixed & Mixed & 6 \\
\hline 4 & 383305122311901 & RITCHEY C NR DEER PARK CA & CA_Ritchey & 38.551424 & -122.521015 & $\mathrm{~N}$ & 6.4 & Undeveloped & Undeveloped & Undeveloped & 4 \\
\hline 76 & 11150500 & SALINAS R NR BRADLEY CA & CA_Salinas & 35.930243 & -120.868791 & S & $6,570.4$ & Undeveloped & Undeveloped & Undeveloped & 6 \\
\hline 49 & 372716122080801 & $\begin{array}{l}\text { SAN FRANCISQUITO C DS } \\
\text { OF NEWELL RD BR A PALO } \\
\text { ALTO }\end{array}$ & CA_SanFranPalo & 37.454383 & -122.136631 & $\mathrm{~N}$ & 99.9 & Urban_med & Urban_high & Urban & 6 \\
\hline 50 & 11164500 & $\begin{array}{l}\text { SAN FRANCISQUITO C A } \\
\text { STANFORD UNIVERSITY CA }\end{array}$ & CA_SanFranStan & 37.423273 & -122.18941 & S & 97.4 & Urban_med & Urban_med & Urban & 6 \\
\hline 43 & 374336122095801 & $\begin{array}{l}\text { SAN LEANDRO C A } \\
\text { ALVARADO ST A SAN } \\
\text { LEANDRO CA }\end{array}$ & CA_SanLeandro & 37.726608 & -122.166178 & $\mathrm{~N}$ & 114.8 & Urban_low & Urban_high & Urban & 6 \\
\hline 81 & 351436120405201 & $\begin{array}{l}\text { SAN LUIS OBISPO C A LOS } \\
\text { OSOS VLY RD NR SAN LUIS } \\
\text { OB }\end{array}$ & CA_SanLObispo & 35.243333 & -120.681111 & S & 107.2 & Urban_low & Urban_med & Urban & 6 \\
\hline 45 & 11181000 & $\begin{array}{l}\text { SAN LORENZO C A HAYWARD } \\
\text { CA }\end{array}$ & CA_SanLorenzo & 37.685486 & -122.064407 & $\mathrm{~N}$ & 97.8 & Urban_low & Urban_med & Urban & 6 \\
\hline 39 & 375220122104201 & $\begin{array}{l}\text { SAN PABLO C A MORAGA } \\
\text { WAY A ORINDA CA }\end{array}$ & CA_SanPabloMor & 37.864508 & -122.172306 & $\mathrm{~N}$ & 4.4 & Urban_med & Urban_med & Urban & 6 \\
\hline 37 & 375312122113501 & SAN PABLO C A ORINDA CA & CA_SanPabloOr & 37.886553 & -122.193119 & $\mathrm{~N}$ & 20.9 & Urban_med & Urban_med & Urban & 6 \\
\hline 34 & 375746122195501 & $\begin{array}{l}\text { SAN PABLO C A EL PORTAL } \\
\text { DR A SAN PABLO CA }\end{array}$ & CA_SanPabloPort & 37.9628 & -122.331997 & $\mathrm{~N}$ & 104.0 & Urban_med & Urban_med & Urban & 6 \\
\hline 40 & 374933122001301 & $\begin{array}{l}\text { SAN RAMON C A LA GONDA } \\
\text { WAY A DANVILLE CA }\end{array}$ & $\begin{array}{l}\text { CA_SanRamon- } \\
\text { Dan }\end{array}$ & 37.825711 & -122.003539 & $\mathrm{~N}$ & 87.5 & Urban_med & Urban_med & Urban & 6 \\
\hline 42 & 11182500 & $\begin{array}{l}\text { SAN RAMON C A SAN RAMON } \\
\text { CA }\end{array}$ & $\begin{array}{l}\text { CA_SanRamon- } \\
\text { San }\end{array}$ & 37.772983 & -121.994682 & $\mathrm{~N}$ & 15.6 & Urban_low & Urban_low & Urban & 6 \\
\hline 13 & 11466200 & $\begin{array}{l}\text { SANTA ROSA C A SANTA } \\
\text { ROSA CA }\end{array}$ & CA_SantaSanta & 38.436579 & -122.72471 & $\mathrm{~N}$ & 144.6 & Urban_low & Urban_high & Urban & 6 \\
\hline
\end{tabular}


Table 1. Characteristics of stream watersheds in the Central California Foothills and Coastal Mountains ecoregion of the United States that were assessed as part of the U.S. Geological Survey National Water-Quality Assessment Project, California Stream Quality Assessment, in 2017._Continued

[Latitude and longitude of water quality sample location based on the North American Datum of 1983 and shown in decimal degrees. The column heading "Northern or Southern group" indicates relative location of sites in the San Francisco Bay area; sampling began the week of March 13, 2017, for the Southern group and April 3, 2017, for the Northern group. Sampling frequency is the number of discrete water samples collected at that site. Abbreviations: ID, identification; $\mathrm{km}^{2}$, square kilometer; NWIS, U.S. Geological Survey National Water Information System database; N, northern; S, southern]

\begin{tabular}{|c|c|c|c|c|c|c|c|c|c|c|c|}
\hline $\begin{array}{l}\text { Map iden- } \\
\text { tification } \\
\text { number1 }\end{array}$ & $\begin{array}{l}\text { NWIS station } \\
\text { number }\end{array}$ & NWIS station name & Field identifier & $\begin{array}{l}\text { Latitude } \\
\text { (NWIS) }\end{array}$ & $\begin{array}{l}\text { Longitude } \\
\text { (NWIS) }\end{array}$ & $\begin{array}{l}\text { Northern } \\
\text { or } \\
\text { Southern } \\
\text { group }\end{array}$ & $\begin{array}{l}\text { Basin } \\
\text { area } \\
\left(\mathrm{km}^{2}\right)\end{array}$ & $\begin{array}{l}\text { Whole basin } \\
\text { land use }\end{array}$ & $\begin{array}{l}\text { Lower basin } \\
\text { land use }{ }^{2}\end{array}$ & $\begin{array}{c}\text { Site type } \\
\text { (lower basin) }^{3}\end{array}$ & $\begin{array}{l}\text { Sampling } \\
\text { frequency }\end{array}$ \\
\hline 9 & 11466320 & $\begin{array}{l}\text { SANTA ROSA C A } \\
\text { WILLOWSIDE RD NR SANTA } \\
\text { ROSA CA }\end{array}$ & CA_SantaWillow & 38.44519 & -122.807213 & $\mathrm{~N}$ & 197.5 & Urban_med & Mixed & Mixed & 6 \\
\hline 84 & 11135250 & $\begin{array}{l}\text { SANTA YNEZ R A 13TH ST } \\
\text { BRIDGE A VAFB NR LOMPOC } \\
\text { CA }\end{array}$ & $\begin{array}{l}\text { CA_SantaYnez- } \\
\text { Lom }\end{array}$ & 34.676617 & -120.553389 & S & $2,283.2$ & Undeveloped & Ag_high & Agriculture & 6 \\
\hline 85 & 11128500 & $\begin{array}{l}\text { SANTA YNEZ R A SOLVANG } \\
\text { CA }\end{array}$ & CA_SantaYnezSol & 34.584987 & -120.144593 & S & $1,498.9$ & Undeveloped & Mixed & Mixed & 6 \\
\hline 56 & 371620122005801 & $\begin{array}{l}\text { SARATOGA C A BRAEMAR DR } \\
\text { A SARATOGA CA }\end{array}$ & CA_Saratoga & 37.272252 & -122.016288 & $\mathrm{~S}$ & 26.2 & Urban_low & Urban_med & Urban & 6 \\
\hline 41 & 374708122132801 & SAUSAL C A OAKLAND CA & CA_Sausal & 37.785681 & -122.224339 & $\mathrm{~N}$ & 10.2 & Urban_med & Urban_high & Urban & 6 \\
\hline 10 & 382634122315201 & $\begin{array}{l}\text { SONOMA C A ADOBE CYN RD } \\
\text { NR KENWOOD CA }\end{array}$ & $\begin{array}{l}\text { CA_SonomaAdo- } \\
\text { be }\end{array}$ & 38.4427 & -122.531 & $\mathrm{~N}$ & 10.3 & Undeveloped & Undeveloped & Undeveloped & 4 \\
\hline 20 & 11458500 & $\begin{array}{l}\text { SONOMA C A AGUA } \\
\text { CALIENTE CA }\end{array}$ & CA_SonomaAgua & 38.323247 & -122.494426 & $\mathrm{~N}$ & 150.5 & Mixed & Mixed & Mixed & 6 \\
\hline 23 & 381556122280201 & $\begin{array}{l}\text { SONOMA C A WATMAUGH RD } \\
\text { BR NR SONOMA CA }\end{array}$ & CA_SonomaWat & 38.265797 & -122.467397 & $\mathrm{~N}$ & 188.0 & Mixed & Mixed & Mixed & 6 \\
\hline 80 & 351725120395901 & $\begin{array}{l}\text { STENNER C A MURRAY AVE A } \\
\text { SAN LUIS OBISPO CA }\end{array}$ & CA_Stenner & 35.2904 & -120.666319 & S & 23.2 & Urban_low & Urban_low & Urban & 6 \\
\hline 25 & 381441122064301 & SUISUN C A ROCKVILLE CA & CA_Suisan & 38.244797 & -122.111997 & $\mathrm{~N}$ & 125.5 & Ag_low & Ag_high & Agriculture & 6 \\
\hline 65 & 365634121264001 & $\begin{array}{l}\text { TEQUISQUITA SLOUGH A } \\
\text { SHORE RD NR DUNNEVILLE } \\
\text { CA }\end{array}$ & CA_Tequisquita & 36.9427 & -121.4445 & S & 301.6 & Ag_low & Ag_high & Agriculture & 6 \\
\hline 16 & 382245122001601 & $\begin{array}{l}\text { ULATIS C A FARRELL RD NR } \\
\text { VACAVILLE CA }\end{array}$ & CA_Ulatis & 38.379169 & -122.004319 & $\mathrm{~N}$ & 27.9 & Ag_low & Mixed & Mixed & 6 \\
\hline 61 & 365955121350601 & $\begin{array}{l}\text { UVAS C A MILLER AVE A } \\
\text { GILROY CA }\end{array}$ & CA_UvasMiller & 36.998533 & -121.584958 & S & 178.7 & Undeveloped & Mixed & Mixed & 6 \\
\hline 36 & 375413122033301 & $\begin{array}{l}\text { WALNUT C A CIVIC DR A } \\
\text { WALNUT CREEK CA }\end{array}$ & CA_Walnut & 37.903669 & -122.059217 & $\mathrm{~N}$ & 204.4 & Urban_med & Urban_high & Urban & 6 \\
\hline 32 & 375808122172601 & $\begin{array}{l}\text { WILKIE C A SANTA RITA RD } \\
\text { NR RICHMOND CA }\end{array}$ & CA_Wilkie & 37.968842 & -122.290553 & $\mathrm{~N}$ & 3.2 & Urban_high & Urban_high & Urban & 6 \\
\hline
\end{tabular}


Table 1. Characteristics of stream watersheds in the Central California Foothills and Coastal Mountains ecoregion of the United States that were assessed as part of the U.S. Geological Survey National Water-Quality Assessment Project, California Stream Quality Assessment, in 2017._Continued

Latitude and longitude of water quality sample location based on the North American Datum of 1983 and shown in decimal degrees. The column heading "Northern or Southern group" indicates relative location of sites in the San Francisco Bay area; sampling began the week of March 13, 2017, for the Southern group and April 3, 2017, for the Northern group. Sampling frequency is the number of discrete water samples collected at that site. Abbreviations: ID, identification; $\mathrm{km}^{2}$, square kilometer; NWIS, U.S. Geological Survey National Water Information System database; N, northern; S, southern]

\begin{tabular}{|c|c|c|c|c|c|c|c|c|c|c|c|}
\hline $\begin{array}{l}\text { Map iden- } \\
\text { tification } \\
\text { number }{ }^{1}\end{array}$ & $\begin{array}{c}\text { NWIS station } \\
\text { number }\end{array}$ & NWIS station name & Field identifier & $\begin{array}{l}\text { Latitude } \\
\text { (NWIS) }\end{array}$ & $\begin{array}{l}\text { Longitude } \\
\text { (NWIS) }\end{array}$ & $\begin{array}{l}\text { Northern } \\
\text { or } \\
\text { Southern } \\
\text { group }\end{array}$ & $\begin{array}{l}\text { Basin } \\
\text { area } \\
\left(\mathbf{k m}^{2}\right)\end{array}$ & $\begin{array}{l}\text { Whole basin } \\
\text { land use }\end{array}$ & $\begin{array}{l}\text { Lower basin } \\
\text { land use }\end{array}$ & $\begin{array}{c}\text { Site type } \\
\text { (lower basin) }\end{array}$ & $\begin{array}{l}\text { Sampling } \\
\text { frequency }\end{array}$ \\
\hline 6 & 383039122502401 & $\begin{array}{l}\text { WINDSOR C A MARK WEST } \\
\text { STATION RD NR WINDSOR } \\
\text { CA }\end{array}$ & CA_Windsor & 38.510957 & -122.839956 & $\mathrm{~N}$ & 65.1 & Mixed & Mixed & Mixed & 6 \\
\hline
\end{tabular}

${ }^{1}$ Map identification number refers to site numbers in figure 3.

2Lower basin land use indicates the dominant land use in the lower part of the watershed extending 5 kilometers from the sampling location. Land-use subcategories are defined in table 3.

${ }^{3}$ Major site type, based on dominant land use in lower basin. Site types are shown in figure 2. 
Watershed delineations and characteristics were available for streams with active USGS streamgages. For other candidate sites, catchment boundaries from the National Hydrography Dataset Plus (NHDPlus Version 2; U.S. Environmental Protection Agency and U.S. Geological Survey, 2012) were used as the watershed boundaries by selecting all upstream catchments from the segment on which the candidate site was located. Nationally available, digital geographic information systems (GIS) data layers (for example, the National Land Cover Database [NLCD], Homer and others, 2015) were overlain on the catchment-derived watersheds, and characteristics of the watersheds were assessed and summarized from these data layers for use during site selection.

After initial screening, based on GIS characteristics and prioritization of gaged and historically monitored sites, the remaining sites were evaluated with the use of satellite imagery (Google Earth ${ }^{\circledR}$, https://www.google.com/earth/) for general watershed characteristics, potential stressor sources (for example, water treatment plants, industrial complexes, golf courses), and accessible sampling reach locations. An objective during this step of site selection was to distribute sites widely across the region and to represent the full range of undeveloped, agricultural, and urban land uses present. This "desk-top reconnaissance" was applied to about 330 sites, of which 129 were selected for field reconnaissance. A field reconnaissance of these sites was conducted by USGS staff during winter and spring 2017. Field observations for each site included evaluating the site for access and safety, assessing general stream characteristics to determine locations for water-quality sampling, and identifying a 150 -meter (m) stream reach with riffle habitat suitable for conducting ecological surveys. Field notes and photography were recorded onsite by using field-reconnaissance forms on an electronic tablet; afterwards, information from these forms was compiled into spreadsheets for use in site review and final selection.

Final site selection was based on the field reconnaissance and on the distribution of land use in the candidate watersheds, again with the objective of representing the range of urban and agricultural development in the region. The distribution of the final 85 sites by land-use type was evaluated at two scales using the NAWQA Wall-to-Wall Anthropogenic Land Use Trends (NWALT) data layers (Falcone, 2015) for whole drainage basins (WB) and for the lower drainage basin only (LB; table 1). The LB boundaries were created by intersecting a 5-kilometer $(\mathrm{km})$ buffer around the sampling site within the drainage basin boundary, consistent with the $5-\mathrm{km}$ scale used by the State of California to assess ecological condition and contamination in streams (Ode and others, 2011). The purpose of using the LB approach was to capture the influence on water quality of land use upstream and close to the sampling location. Some headwater streams flow from relatively undeveloped hills of the California Coast
Ranges into an intensely developed valley. In such cases, the WB characteristics might indicate a relatively undeveloped basin, even though the site might be strongly affected by proximal development.

The NWALT data provided a more detailed delineation of urban land uses than is available in NLCD (U.S. Geological Survey, 2014). A two-step process was applied, starting with the NWALT data, to assign a land-use category to each site. NWALT land-use "classes" (as described in Falcone, 2015, such as high density residential developed and cultivated crops) were combined into summed land-use "types" (such as Total Urban or Total Agriculture) to represent the types and degree of urban development and agriculture in the watersheds of sites (table 2). Each site was then assigned to a land-use category (Urban, Agriculture, Mixed, or Undeveloped) and a "land-use subcategory" (such as Urban_high, Ag_high, Undeveloped) on the basis of combinations and threshold levels of the various land-use types in the watershed (table 3). On the basis of visual inspections of the basins in satellite imagery (Google Earth ${ }^{\circledR}$ ), NWALT data occasionally appeared to misrepresent some types of agricultural land in California; specifically, nurseries, greenhouses, orchards, and vineyards were classified in some cases as urban or semi-developed land. Such apparent errors were confirmed by consulting the Statewide Crop Mapping 2014 dataset (Land IQ, LLC, 2017), which is the official dataset of the California Department of Water Resources (CDWR) for determining agricultural land use and irrigated acres (California Department of Water Resources, 2019) and which indicated that the percentage of agricultural land was underestimated in some basins. In these cases, the assigned land-use category was adjusted to reflect the visual characteristics (such crop fields, greenhouses, orchards) and cropping patterns from the CDWR dataset.

The final 85 sites are widely distributed across the region (fig. 2). Individual site locations are shown in fig. 3, numbered in relation to site names in table 1 . There were more mixed sites and fewer undeveloped sites for watershed delineations at the LB scale than at the WB scale (table 4), possibly because there was generally more undeveloped land in the upper basin. This intensification of development in the vicinity of the sampling site is seen by comparing plots of the WB and LB of site distributions in relation to two primary land-use types: total urban and cropland (fig. 4). In each of the WB and LB delineations, a large subset of sites represented a full urban gradient reasonably well, without the complicating factor of high levels of agricultural land use also within the watersheds. More mixed land-use sites were apparent at the LB scale (fig. 4; table 4). Of the 85 sites, 39 were classified as having some degree of urban land use at both the LB and WB scales; however, only 5 sites were classified as Agricultural sites at both the LB and WB scales. Although there is extensive agriculture in some watersheds in the region, most of it is mixed with urban development. 
Table 2. Land-use classes from the National Water Quality Assessment wall-to-wall anthropogenic land use trends database were summed to create land-use types.

[The parenthetical numbers are the assigned numeric class designations from the National Water Quality Assessment wall-to-wall anthropogenic land use trends (NWALT) dataset (Falcone, 2015). Thresholds were applied to these land-use types to assign each site to a land-use category (as in table 3). Abbreviation: - , not applicable]

\begin{tabular}{|c|c|c|c|c|c|c|c|}
\hline \multirow[b]{2}{*}{ NWALT land-use classes 1} & \multicolumn{7}{|c|}{ Land-use types } \\
\hline & $\begin{array}{l}\text { Dense } \\
\text { urban }\end{array}$ & $\begin{array}{c}\text { Total } \\
\text { urban }\end{array}$ & $\begin{array}{c}\text { Extended } \\
\text { urban }\end{array}$ & Cropland & $\begin{array}{c}\text { Total } \\
\text { agriculture }\end{array}$ & $\begin{array}{c}\text { Total } \\
\text { developed }\end{array}$ & $\begin{array}{l}\text { Low } \\
\text { usage }\end{array}$ \\
\hline Wetlands (12) & - & - & - & - & - & - & $\mathrm{X}$ \\
\hline Dev_MajorTransp (21) & $\mathrm{X}$ & $\mathrm{X}$ & $\mathrm{X}$ & - & - & $\mathrm{X}$ & - \\
\hline Dev_Recreation (24) & - & $\mathrm{X}$ & $\mathrm{X}$ & - & - & $\mathrm{X}$ & - \\
\hline Dev_ResidHigh (25) & - & $\mathrm{X}$ & $\mathrm{X}$ & - & - & $\mathrm{X}$ & - \\
\hline Dev_ResidLowMed (26) & - & $\mathrm{X}$ & $\mathrm{X}$ & - & - & $\mathrm{X}$ & - \\
\hline Dev_Other (27) & - & $\mathrm{X}$ & $\mathrm{X}$ & - & - & $\mathrm{X}$ & - \\
\hline Prod_Mining (41) & - & - & - & - & - & $\mathrm{X}$ & - \\
\hline Prod_Crops (43) & - & - & - & $\mathrm{X}$ & $\mathrm{X}$ & $\mathrm{X}$ & - \\
\hline Prod_PastureHay (44) & - & - & - & - & $\mathrm{X}$ & $\mathrm{X}$ & - \\
\hline Prod_GrazingPot (45) & - & - & - & - & $\mathrm{X}$ & $\mathrm{X}$ & - \\
\hline LowUse (50) & - & - & - & - & - & - & $\mathrm{X}$ \\
\hline VeryLowUse_Conserv (60) & - & - & - & - & - & - & $\mathrm{X}$ \\
\hline
\end{tabular}

1Dev_CommServices, developed, commercial/services, class 22; Dev_IndusMilitary, developed, industrial/military, class 23; Dev_MajorTransp, developed, major transportation, class 21; Dev_Other, developed, other, class 27; Dev_Recreation, developed, recreation, class 24; Dev_ResidHigh, developed, residential high density, class 25; Dev_ResidLowMed, developed, residential low-medium density, class 26; SemiDev_AnthroOth, semi-developed, anthropogenic other, class 33; SemiDev_UrbHigh, semideveloped, urban interface high, class 31; SemiDev_UrbLowMed, semi-developed, urban interface low-medium, class 32; Prod_Crops, production, crops, class 43; Prod_GrazingPot, production, grazing potential, class 45; Prod_Mining, production, mining/extraction, class 41; Prod_ PastureHay, production, pasture/hay, class 44; LowUse, no other land use, class 50; VeryLowUse_Conserv, no other land use and in a protected area, conservation, class 60; Water, water, class 11; Wetlands, wetlands, class 12.

${ }^{2}$ For the California Stream Quality Assessment study, total urban excluded the SemiDev_UrbHigh class because in California, this NWALT class sometimes mischaracterized agricultural land containing scattered buildings (such as vineyards, nurseries) as semi-developed urban high. In other Regional Stream Quality Assessment studies, however, total urban included the SemiDev_UrbHigh class. 
Table 3. Thresholds and combinations of land-use types used to assign sites to unique land-use categories.

[Land-use types used in the conditions below are defined in table 2, and are based on land-use data from the National Water Quality Assessment wall-to-wall anthropogenic land use trends (NWALT) dataset (Falcone, 2015). Abbreviations: >, greater than; \%, percent; <, less than; - , not applicable]

\begin{tabular}{|c|c|c|c|}
\hline \multirow{2}{*}{$\begin{array}{c}\text { Land use } \\
\text { subcategory }\end{array}$} & \multicolumn{3}{|c|}{ Conditions (all must be met, except as indicated in OR statements) } \\
\hline & Urban AND & Agriculture AND & Other \\
\hline \multicolumn{4}{|c|}{ Major land use category: Urban } \\
\hline Urban_med & $25-75 \%$ Total urban & $<10 \%$ Cropland AND $<25 \%$ Total agriculture & - \\
\hline Urban_low & $5-25 \%$ Total urban OR > 25\% Extended urban & $<10 \%$ Cropland AND $<25 \%$ Total agriculture & Total urban $>$ Cropland \\
\hline Ag_high & $<10 \%$ Total urban AND $<25 \%$ Extended urban & $>25 \%$ Cropland & - \\
\hline Ag_low & $<10 \%$ Total urban AND $<25 \%$ Extended urban & $5-25 \%$ Cropland OR $>25 \%$ Total agriculture & Crops $>$ Total urban \\
\hline \multicolumn{4}{|c|}{ Major land use category: Mixed } \\
\hline Mixed & $>10 \%$ Total urban OR $>25 \%$ Extended urban & $>10 \%$ Cropland OR $>25 \%$ Total agriculture & - \\
\hline \multicolumn{4}{|c|}{ Major land use category: Undeveloped } \\
\hline
\end{tabular}

Table 4. Distribution of 85 California Stream Quality Assessment sites by land-use category for the whole basin and lower basin watershed delineations.

[Land-use categories are defined in table 3.]

\begin{tabular}{lcc}
\hline Land-use category & Whole basin & Lower basin \\
\hline Urban_high & 4 & 15 \\
Urban_med & 15 & 20 \\
Urban_low & 24 & 5 \\
Ag_high & 1 & 5 \\
Ag_low & 11 & 4 \\
Mixed & 13 & 24 \\
Undeveloped & 17 & 12 \\
\hline
\end{tabular}




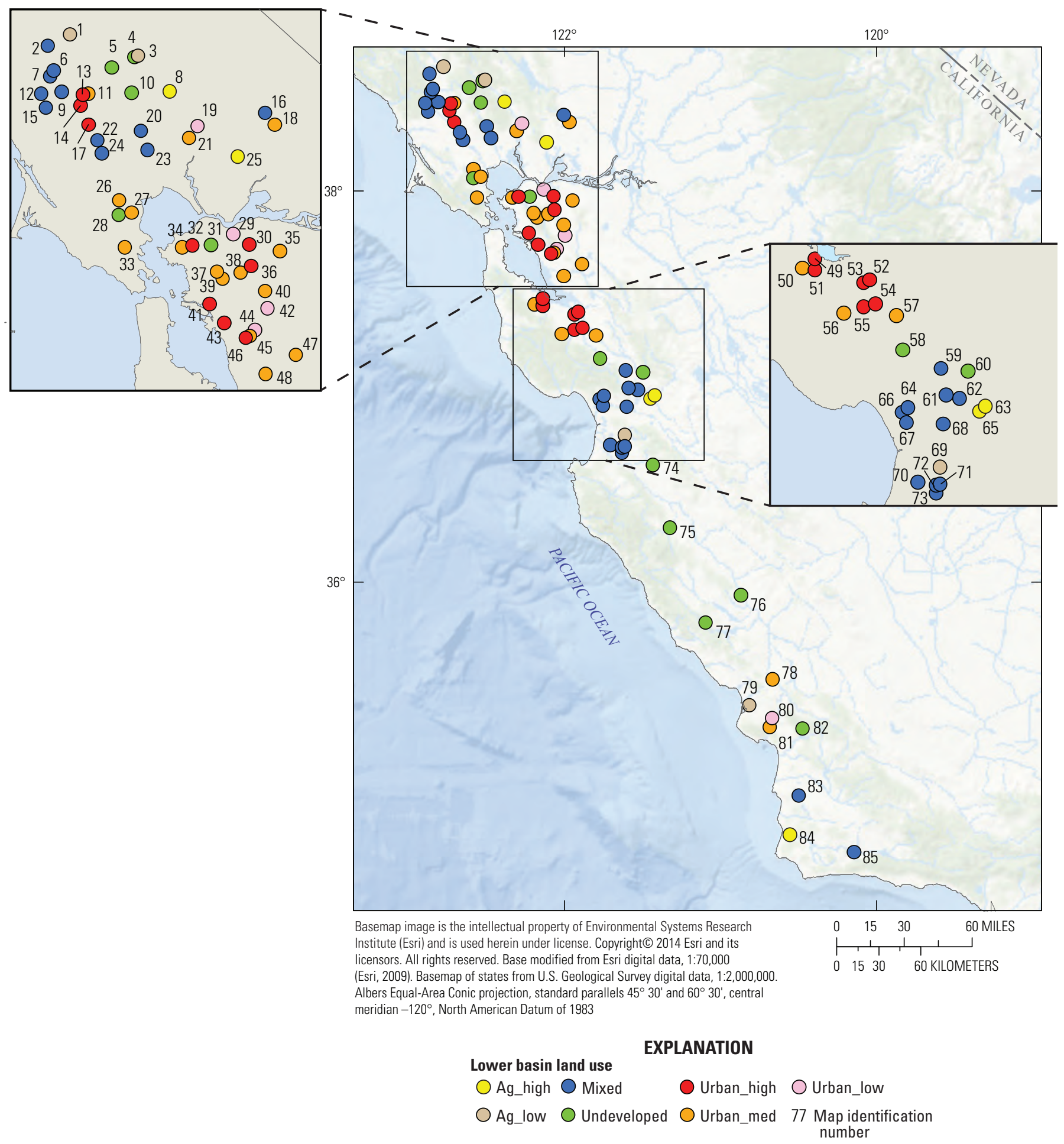

Figure 3. Locations of the 85 sites sampled for the California Stream Quality Assessment study, 2017. Sites are color-coded by lower basin land-use subcategory (as defined in table 3). Site names are listed by map identification number in table 1. 

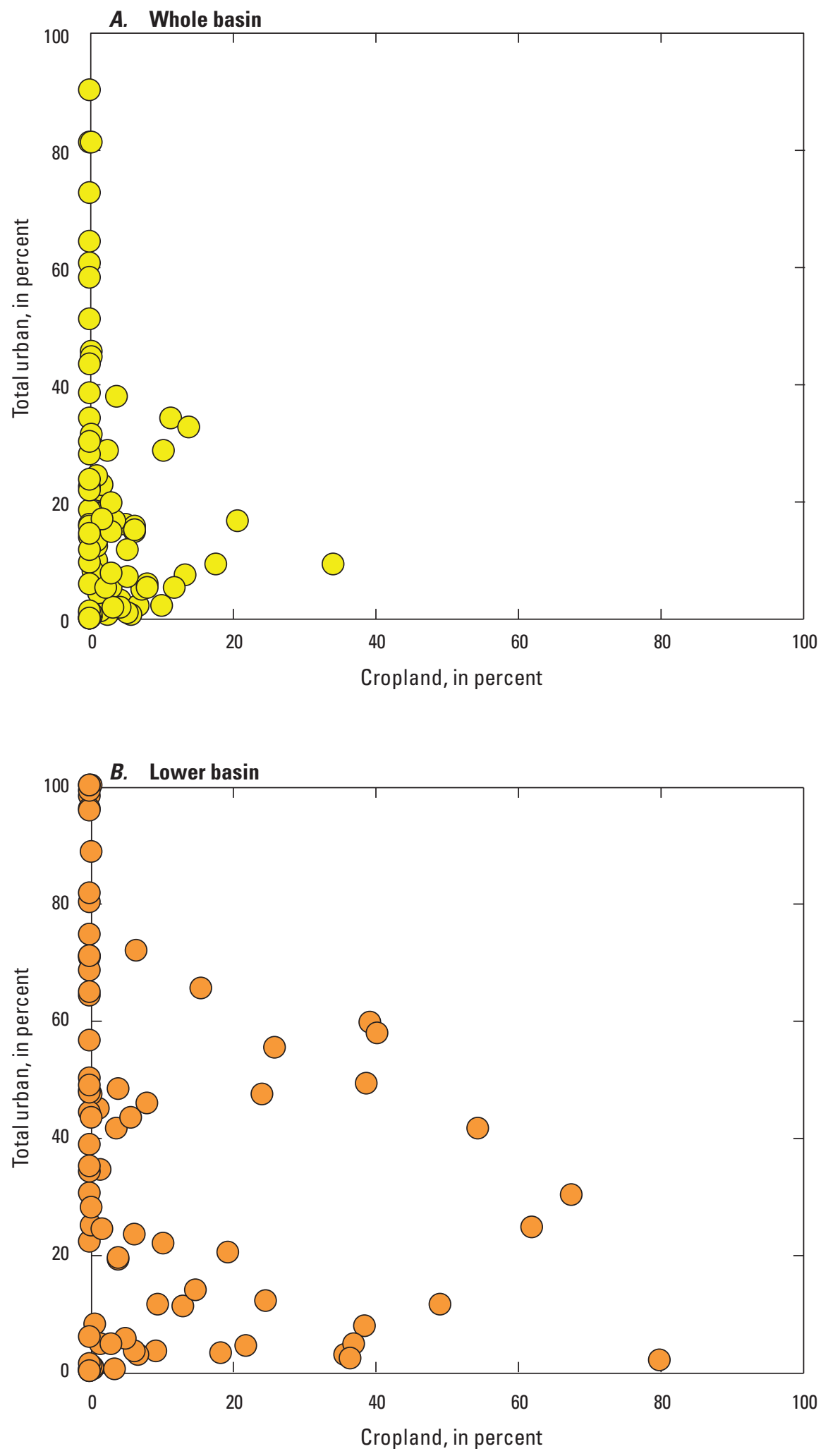

Figure 4. Distribution of 85 California Stream Quality Assessment sites in relation to percent of cropland and percent of total urban (as defined in table 2) for $A$, the whole basin; and $B$, lower basin watershed delineation approaches. Data are from $0 i$ and Nakagaki (2020). 


\section{Sample Collection and Processing}

The types of data and the intervals at which samples were collected varied among the sites according to land-cover type and associated potential stressors. Data collection and sampling routines were categorized nominally by three study-design components (table 5). The comprehensive stressor assessments identify the various water-quality constituents that were collected at all sites. Although the study design called for measuring streamflow or stage and continuous temperature at all sites, some sites are missing stage or temperature data because pressure or temperature sensors were either not deployed or were lost in the field owing to high flow conditions. The focused studies component was composed of limited investigations at subsets of streams that focused on the occurrence and timing of specific stressors potentially affecting the condition of those streams. The ecological surveys component identified sampling activities associated with the ecological surveys done at the end of the index period at all sites.

The various constituents in each study design component are characterized in table 5 by the time interval at which they were collected: discrete, integrated, or continuous. Discrete samples characterized stream conditions at a specific point in time and could be collected at multiple times during the study (for example, "Basic quality of water (QW)" constituents, table 5) or collected only once during the study (for example, "N and $\mathrm{O}$ isotopes," table 5). Integrated data were collected by passive sampling devices that were deployed during the study to represent an average or cumulative condition of the stream (for example, polar organic chemical integrative sampler; POCIS, table 5). Continuous data (such as streamflow, stage, temperature; table 5) were recorded at 15-minute intervals. All constituents included in Ecological Surveys (table 5) were measured once to represent conditions at a point in time and, therefore, the data are discrete; however, because these constituents generally acclimate to environmental variations over time, they typically reflect integrated conditions.

USGS staff who participated in the CSQA study received training before data collection activities, including instructions specific to water-quality sampling, focused studies, and ecological surveys. For example, the use of low-level analytical methods necessitated that water samples be collected according to "parts-per-billion" protocols (U.S. Geological Survey, 2006). During weeks when water-quality data were collected at all 85 sites, nine 2-person teams of USGS staff were deployed. To ensure consistency among the water-quality teams, training for the collection and processing of water-quality samples occurred in February 2017 for all personnel involved with sample collection. Classroom water-quality training was followed by field-training exercises to work through all sampling and processing procedures in the field before the start of sampling. The sample collection timelines, sample types collected at sites, and sample collection, processing, and handling procedures are summarized in appendix 1 and described briefly here.

Ecological survey teams were trained in consistent sampling protocols, including State of California protocols for the collection of algae and aquatic macroinvertebrates (Ode and others, 2016), and USGS protocols for habitat surveys (Fitzpatrick and others, 1998) and bed-sediment collection (Shelton and Capel, 1994; Radtke, 2005). Before beginning the sampling trip, participants gathered at a local stream and instructors demonstrated the various protocols for collecting each sample type. The participants completed a full ecological collection at the training location. The ecological sampling coordinator visited each 5-person team during the sampling trip to verify techniques and clarify any questions.

\section{Comprehensive Stressor Assessments}

To allocate resources effectively among the 85 sites, various water-quality constituents were sampled at different frequencies during the study; the differences in sampling among sites generally were based on intensities of watershed development. The WQ index period, described in the "Background" section, was 6 weeks for 74 of the 85 sites and 4 weeks for the other 11 sites (all of which were classified as "Undeveloped" at both the WB and LB scales). These undeveloped sites were sampled during the last 4 weeks of the 6-week study period.

The 6-week WQ index period was March 13 to April 21, 2017, for the Southern sites, and April 3 to May 12 for the Northern sites. Details of the sampling schedule indicating the weeks that constituents were sampled at individual sites are provided in appendix 1, table 1.1. 
Table 5. Summary of data collected at the California Stream Quality Assessment sites in 2017.

[Constituents are categorized by the study-design components: Comprehensive Stressor Assessments, Focused Studies, and Ecological Surveys. Notes and abbreviations: See footnote 1 and text for Basic quality of water (QW) constituents. Abbreviations: C, continuous sample; D, discrete samples; I, integrative sample. Numerical value in cell indicates number of discrete samples collected: x, data or sample collected; - , not sampled. Streamflow or stage: Egage, gaging station for an external agency other than U.S. Geological Survey (USGS); Gage, USGS gaging station; PT, pressure transducer installed to record stream stage. Ecological Surveys were conducted once at sites near the end of the water-quality index period: Aquatic Biota, invertebrate and algal samples collected; Streambed

Sediment: Tx, two temporal samples were collected prior to collecting sediment during ecological survey; Sediment Toxicity Test, sediment tested on survival of invertebrates: Ch, Chironomus larvae; Hy, Hyalella. Other abbreviations: N, nitrogen; NWIS; U.S. Geological Survey National Water Information System database; O, oxygen; POCIS, polar organic chemical integrative sampler; SS, suspended sediment]

\begin{tabular}{|c|c|c|c|c|c|c|c|c|c|c|c|c|c|c|c|c|c|c|}
\hline \multicolumn{2}{|c|}{ Site information } & \multicolumn{10}{|c|}{ Comprehensive stressor assessments } & \multicolumn{3}{|c|}{ Focused studies } & \multicolumn{4}{|c|}{ Ecological surveys } \\
\hline $\begin{array}{c}\text { NWIS station } \\
\text { number }\end{array}$ & Field identifier & $\begin{array}{c}\text { Basic } \\
\text { QW1 } \\
\text { (D) }\end{array}$ & $\begin{array}{l}\text { Mer- } \\
\text { cury } \\
\text { (D) }\end{array}$ & $\begin{array}{c}\text { Organic } \\
\text { waste } \\
\text { indica- } \\
\text { tors } \\
\text { (D) }\end{array}$ & $\begin{array}{l}\text { Pharma- } \\
\text { ceuticals } \\
\text { (D) }\end{array}$ & $\begin{array}{l}\text { Glypho- } \\
\text { sate } \\
\text { analysis } \\
\text { (D) }\end{array}$ & $\begin{array}{c}\mathrm{N} \text { and } \mathbf{0} \\
\text { iso- } \\
\text { topes } \\
\text { (D) }\end{array}$ & $\begin{array}{l}\text { Algal } \\
\text { toxins } \\
\text { (D) }\end{array}$ & $\begin{array}{l}\text { POCIS } \\
(\text { I })^{2}\end{array}$ & $\begin{array}{l}\text { Stream- } \\
\text { flow or } \\
\text { stage } \\
\text { (C) }\end{array}$ & $\begin{array}{l}\text { Temp- } \\
\text { erature } \\
\text { (C) }\end{array}$ & $\begin{array}{c}\text { Pest- } \\
\text { icide } \\
\text { auto- } \\
\text { sampler } \\
\text { (I) }\end{array}$ & $\begin{array}{c}\text { Biofilm } \\
\text { pesti- } \\
\text { cides } \\
\text { (I) }\end{array}$ & $\begin{array}{c}\text { SS } \\
\text { passive } \\
\text { sam- } \\
\text { pler } \\
\text { (I) }\end{array}$ & $\begin{array}{l}\text { Aquatic } \\
\text { biota } \\
\text { (D) }\end{array}$ & $\begin{array}{l}\text { Physi- } \\
\text { cal } \\
\text { habitat } \\
\text { (D) }\end{array}$ & $\begin{array}{l}\text { Stream- } \\
\text { bed } \\
\text { sedi- } \\
\text { ment } \\
\text { (D) }\end{array}$ & $\begin{array}{c}\text { Sedi- } \\
\text { ment } \\
\text { toxicity } \\
\text { test } \\
\text { (D) }\end{array}$ \\
\hline 11179100 & CA_Alameda & 6 & 3 & 3 & 3 & 2 & 1 & 1 & - & Gage & - & - & - & - & $\mathrm{x}$ & $\mathrm{x}$ & $\mathrm{x}$ & $\mathrm{HyCh}$ \\
\hline 382035121575501 & CA_Alamo & 6 & 3 & 3 & 3 & 2 & 1 & 1 & - & Egage & - & - & $\mathrm{x}$ & - & $\mathrm{x}$ & $\mathrm{x}$ & $\mathrm{x}$ & $\mathrm{HyCh}$ \\
\hline 364003121373501 & CA_Alisal & 6 & 3 & 3 & 3 & 2 & 1 & 1 & - & - & $\mathrm{x}$ & - & - & - & $\mathrm{x}$ & $\mathrm{x}$ & $\mathrm{x}$ & Нy \\
\hline 11176900 & CA_ArroyoDeLaLa & 6 & 3 & 3 & 3 & 2 & 1 & 1 & $\mathrm{x}$ & Gage & $\mathrm{x}$ & - & $\mathrm{x}$ & - & $\mathrm{x}$ & $\mathrm{x}$ & $\mathrm{x}$ & $\mathrm{HyCh}$ \\
\hline 380410122315501 & CA_ArroyoDig & 6 & 3 & 3 & 3 & 2 & 1 & 1 & $\mathrm{x}$ & PT & $\mathrm{x}$ & - & $\mathrm{x}$ & - & $\mathrm{x}$ & $\mathrm{x}$ & $\mathrm{x}$ & $\mathrm{HyCh}$ \\
\hline 11182400 & CA_ArroyoMart & 6 & 3 & 3 & 3 & 2 & 1 & 1 & $\mathrm{x}$ & - & - & - & $\mathrm{x}$ & - & $\mathrm{x}$ & $\mathrm{x}$ & $\mathrm{x}$ & $\mathrm{HyCh}$ \\
\hline 380345122345201 & CA_ArroyoNov & 4 & 2 & 1 & 1 & - & 1 & 1 & $\mathrm{x}$ & - & - & - & $\mathrm{x}$ & - & $\mathrm{x}$ & $\mathrm{x}$ & $\mathrm{x}$ & $\mathrm{HyCh}$ \\
\hline 11152000 & CA_ArroyoSeco & 4 & 2 & 1 & 1 & - & 1 & 1 & - & Gage & $\mathrm{x}$ & - & $\mathrm{x}$ & - & $\mathrm{x}$ & $\mathrm{x}$ & $\mathrm{x}$ & Hy \\
\hline 352934120395501 & CA_Atascadero & 6 & 3 & 3 & 3 & 2 & 1 & 1 & $\mathrm{x}$ & - & $\mathrm{x}$ & - & $\mathrm{x}$ & - & $\mathrm{x}$ & $\mathrm{x}$ & $\mathrm{x}$ & Hy \\
\hline 11181008 & CA_Castro & 6 & 3 & 3 & 3 & 2 & 1 & 1 & $\mathrm{x}$ & Gage & - & $\mathrm{L}$ & - & $\mathrm{x}$ & $\mathrm{x}$ & $\mathrm{x}$ & $\mathrm{x}$ & $\mathrm{HyCh}$ \\
\hline 352127120484501 & CA_Chorro & 6 & 3 & 3 & 3 & 2 & 1 & 1 & $\mathrm{x}$ & PT & $\mathrm{x}$ & - & - & - & $\mathrm{x}$ & $\mathrm{x}$ & $\mathrm{x}$ & Нy \\
\hline 363608121255201 & CA_ChuChuChu & 4 & 2 & 1 & 1 & - & 1 & 1 & $\mathrm{x}$ & - & - & - & $\mathrm{x}$ & - & $\mathrm{x}$ & $\mathrm{x}$ & $\mathrm{x}$ & Нy \\
\hline 11465690 & CA_Colgan & 6 & 3 & 3 & 3 & 2 & 1 & 1 & $\mathrm{x}$ & Gage & $\mathrm{x}$ & - & $\mathrm{x}$ & - & $\mathrm{x}$ & $\mathrm{x}$ & Tx & $\mathrm{HyCh}$ \\
\hline 11456500 & CA_ConnOak & 6 & 3 & 3 & 3 & 2 & 1 & 1 & $\mathrm{x}$ & Gage & - & $\mathrm{x}$ & $\mathrm{x}$ & - & $\mathrm{x}$ & $\mathrm{x}$ & $\mathrm{x}$ & $\mathrm{HyCh}$ \\
\hline 11465660 & CA_Copeland & 6 & 3 & 3 & 3 & 2 & 1 & 1 & $\mathrm{x}$ & Gage & $\mathrm{x}$ & - & $\mathrm{x}$ & - & $\mathrm{x}$ & $\mathrm{x}$ & Tx & Hy \\
\hline 11159200 & CA_Corralitos & 6 & 3 & 3 & 3 & 2 & 1 & 3 & - & Gage & $\mathrm{x}$ & - & $\mathrm{x}$ & - & $\mathrm{x}$ & $\mathrm{x}$ & $\mathrm{x}$ & Hy \\
\hline 11460000 & CA_Corte & 6 & 3 & 3 & 3 & 2 & 1 & 1 & $\mathrm{x}$ & Gage & $\mathrm{x}$ & - & $\mathrm{x}$ & - & $\mathrm{x}$ & $\mathrm{x}$ & $\mathrm{x}$ & $\mathrm{HyCh}$ \\
\hline 372303121542901 & CA_CoyoteChar & 6 & 3 & 3 & 3 & 2 & 1 & 1 & - & - & - & $\mathrm{x}$ & - & - & $\mathrm{x}$ & $\mathrm{x}$ & Tx & $\mathrm{HyCh}$ \\
\hline 371554121474101 & CA_CoyoteCoyote & 6 & 3 & 3 & 3 & 2 & 1 & 1 & $\mathrm{x}$ & - & - & - & $\mathrm{x}$ & - & $\mathrm{x}$ & $\mathrm{x}$ & $\mathrm{x}$ & Hy \\
\hline 11169800 & CA_CoyoteGil & 4 & 2 & 1 & 1 & - & 1 & 1 & $\mathrm{x}$ & Gage & $\mathrm{x}$ & - & - & - & $\mathrm{x}$ & $\mathrm{x}$ & $\mathrm{x}$ & Нy \\
\hline 11180900 & CA_Crow & 6 & 3 & 3 & 3 & 2 & 1 & 1 & $\mathrm{x}$ & Gage & - & - & - & $\mathrm{x}$ & $\mathrm{x}$ & $\mathrm{x}$ & $\mathrm{x}$ & $\mathrm{HyCh}$ \\
\hline 11465350 & CA_DryMouth & 6 & 3 & 3 & 3 & 2 & 1 & 1 & $\mathrm{x}$ & Gage & - & - & - & - & - & - & - & - \\
\hline 364138121373701 & CA_GabilanEastL & 6 & 3 & 3 & 3 & 2 & 1 & 1 & $\mathrm{x}$ & - & $\mathrm{x}$ & - & $\mathrm{x}$ & - & $\mathrm{x}$ & $\mathrm{x}$ & $\mathrm{x}$ & Ну \\
\hline 11152600 & CA_GabilanSal & 6 & 3 & 3 & 3 & 2 & 1 & 1 & $\mathrm{x}$ & - & $\mathrm{x}$ & - & - & - & $\mathrm{x}$ & $\mathrm{x}$ & $\mathrm{x}$ & Hy \\
\hline 375819122035801 & CA_Grayson & 6 & 3 & 3 & 3 & 2 & 1 & 1 & $\mathrm{x}$ & - & - & - & - & - & $\mathrm{x}$ & $\mathrm{x}$ & Tx & $\mathrm{HyCh}$ \\
\hline 382619122531401 & CA_Green & 6 & 3 & 3 & 3 & 2 & 1 & 1 & $\mathrm{x}$ & - & - & - & $\mathrm{x}$ & - & $\mathrm{x}$ & $\mathrm{x}$ & $\mathrm{x}$ & Hy \\
\hline 11169025 & CA_GuadalupeA & 6 & 3 & 3 & 3 & 2 & 1 & 1 & $\mathrm{x}$ & Gage & - & - & - & - & $\mathrm{x}$ & $\mathrm{x}$ & $\mathrm{x}$ & $\mathrm{HyCh}$ \\
\hline 371814121525601 & CA_GuadalupeB & 6 & 3 & 3 & 3 & 2 & 1 & 1 & - & - & - & - & - & - & $\mathrm{x}$ & $\mathrm{x}$ & $\mathrm{x}$ & $\mathrm{HyCh}$ \\
\hline 375257122050001 & CA_LasTrampas & 6 & 3 & 3 & 3 & 2 & 1 & 1 & $\mathrm{x}$ & - & - & - & $\mathrm{x}$ & - & $\mathrm{x}$ & $\mathrm{x}$ & $\mathrm{x}$ & $\mathrm{HyCh}$ \\
\hline 381740122395901 & CA_Lichau & 6 & 3 & 3 & 3 & 2 & 1 & 1 & $\mathrm{x}$ & PT & $\mathrm{x}$ & - & $\mathrm{x}$ & - & $\mathrm{x}$ & $\mathrm{x}$ & $\mathrm{x}$ & Hy \\
\hline 11153650 & CA_LlagasGilroy & 6 & 3 & 3 & 3 & 2 & 1 & 3 & $\mathrm{x}$ & Gage & - & - & $\mathrm{x}$ & - & $\mathrm{x}$ & $\mathrm{x}$ & Tx & Hy \\
\hline 370512121361901 & CA_LlagasMartin & 6 & 3 & 3 & 3 & 2 & 1 & 3 & $\mathrm{x}$ & PT & $\mathrm{x}$ & - & - & - & $\mathrm{x}$ & $\mathrm{x}$ & $\mathrm{x}$ & Hy \\
\hline 11153470 & CA_LlagasMorgan & 4 & 2 & 1 & 1 & - & 1 & 1 & $\mathrm{x}$ & - & - & - & - & - & $\mathrm{x}$ & $\mathrm{x}$ & $\mathrm{x}$ & Нy \\
\hline 11141280 & CA_Lopez & 4 & 2 & 1 & 1 & - & 1 & 1 & $\mathrm{x}$ & Gage & $\mathrm{x}$ & - & $\mathrm{x}$ & - & $\mathrm{x}$ & $\mathrm{x}$ & $\mathrm{x}$ & Hy \\
\hline 371738121555901 & CA_LosGatos & 6 & 3 & 3 & 3 & 2 & 1 & 1 & $\mathrm{x}$ & - & - & - & $\mathrm{x}$ & - & $\mathrm{x}$ & $\mathrm{x}$ & $\mathrm{x}$ & $\mathrm{HyCh}$ \\
\hline 383719122462501 & CA_Maacama & 6 & 3 & 3 & 3 & 2 & 1 & 1 & $\mathrm{x}$ & PT & $\mathrm{x}$ & - & $\mathrm{x}$ & - & $\mathrm{x}$ & $\mathrm{x}$ & $\mathrm{x}$ & Hy \\
\hline
\end{tabular}


[Constituents are categorized by the study-design components: Comprehensive Stressor Assessments, Focused Studies, and Ecological Surveys. Notes and abbreviations: See footnote 1 and text for Basic quality of water (QW) constituents. Abbreviations: C, continuous sample; D, discrete samples; I, integrative sample. Numerical value in cell indicates number of discrete samples collected: $\mathrm{x}$, data or sample collected; - , not sampled. Streamflow or stage: Egage, gaging station for an external agency other than U.S. Geological Survey (USGS); Gage, USGS gaging station; PT, pressure transducer installed to record stream stage. Ecological Surveys were conducted once at sites near the end of the water-quality index period: Aquatic Biota, invertebrate and algal samples collected; Streambed Sediment: Tx, two temporal samples were collected prior to collecting sediment during ecological survey; Sediment Toxicity Test, sediment tested on survival of invertebrates: Ch, Chironomus larvae; Hy, Hyalella. Other abbreviations: N, nitrogen; NWIS; U.S. Geological Survey National Water Information System database; O, oxygen; POCIS, polar organic chemical integrative sampler; SS, suspended sediment]

\begin{tabular}{|c|c|c|c|c|c|c|c|c|c|c|c|c|c|c|c|c|c|c|}
\hline \multicolumn{2}{|c|}{ Site information } & \multicolumn{10}{|c|}{ Comprehensive stressor assessments } & \multicolumn{3}{|c|}{ Focused studies } & \multicolumn{4}{|c|}{ Ecological surveys } \\
\hline $\begin{array}{c}\text { NWIS station } \\
\text { number }\end{array}$ & Field identifier & $\begin{array}{c}\text { Basic } \\
\text { OW1 } \\
\text { (D) }\end{array}$ & $\begin{array}{l}\text { Mer- } \\
\text { cury } \\
\text { (D) }\end{array}$ & $\begin{array}{l}\text { Organic } \\
\text { waste } \\
\text { indica- } \\
\text { tors } \\
\text { (D) }\end{array}$ & $\begin{array}{l}\text { Phar- } \\
\text { ma- } \\
\text { ceuti- } \\
\text { cals } \\
\text { (D) }\end{array}$ & $\begin{array}{l}\text { Glypho- } \\
\text { sate } \\
\text { analysis } \\
\text { (D) }\end{array}$ & $\begin{array}{l}\mathrm{N} \text { and } \mathbf{0} \\
\text { iso- } \\
\text { topes } \\
\text { (D) }\end{array}$ & $\begin{array}{l}\text { Algal } \\
\text { toxins } \\
\text { (D) }\end{array}$ & $\begin{array}{l}\text { POCIS } \\
(\text { I })^{2}\end{array}$ & $\begin{array}{l}\text { Stream- } \\
\text { flow or } \\
\text { stage } \\
\text { (C) }{ }^{3}\end{array}$ & $\begin{array}{c}\text { Tem- } \\
\text { pera- } \\
\text { ture } \\
\text { (C) }\end{array}$ & $\begin{array}{l}\text { Pes- } \\
\text { ticide } \\
\text { auto- } \\
\text { sampler } \\
\text { (I) }\end{array}$ & $\begin{array}{l}\text { Bio- } \\
\text { film } \\
\text { pesti- } \\
\text { cides } \\
\text { (I) }\end{array}$ & $\begin{array}{c}\text { SS } \\
\text { passive } \\
\text { sam- } \\
\text { pler } \\
\text { (I) }\end{array}$ & $\begin{array}{l}\text { Aquat- } \\
\text { ic biota } \\
\text { (D) }\end{array}$ & $\begin{array}{l}\text { Physi- } \\
\text { cal } \\
\text { habitat } \\
\text { (D) }\end{array}$ & $\begin{array}{l}\text { Stream- } \\
\text { bed } \\
\text { sedi- } \\
\text { ment } \\
\text { (D) }\end{array}$ & $\begin{array}{c}\text { Sedi- } \\
\text { ment } \\
\text { toxicity } \\
\text { test } \\
\text { (D) }\end{array}$ \\
\hline 11466800 & CA_MarkWMir & 6 & 3 & 3 & 3 & 2 & 1 & 1 & $\mathrm{x}$ & Gage & $\mathrm{x}$ & - & - & - & - & - & - & - \\
\hline 383109122363301 & CA MarkWTar & 4 & 2 & 1 & 1 & - & 1 & 1 & $\mathrm{x}$ & - & - & - & $\mathrm{x}$ & - & $\mathrm{x}$ & $\mathrm{x}$ & $\mathrm{x}$ & $\mathrm{HyCh}$ \\
\hline 372500122081201 & $\mathrm{CA}^{-}$Matadero & 6 & 3 & 3 & 3 & 2 & 1 & 1 & $\mathrm{x}$ & Gage & $\mathrm{x}$ & - & $\mathrm{x}$ & - & $\mathrm{x}$ & $\mathrm{x}$ & $\mathrm{x}$ & $\mathrm{HyCh}$ \\
\hline 11466170 & CA_Matanzas & 6 & 3 & 3 & 3 & 2 & 1 & 1 & $\mathrm{x}$ & Gage & $\mathrm{x}$ & $\mathrm{x}$ & $\mathrm{x}$ & - & $\mathrm{x}$ & $\mathrm{x}$ & $\mathrm{x}$ & $\mathrm{HyCh}$ \\
\hline 382017122161101 & CA_Milliken & 6 & 3 & 3 & 3 & 2 & 1 & 1 & $\mathrm{x}$ & PT & $\mathrm{x}$ & - & $\mathrm{x}$ & - & $\mathrm{x}$ & $\mathrm{x}$ & $\mathrm{x}$ & Нy \\
\hline 375701121564401 & $\mathrm{CA}^{-} \mathrm{MtDiablo}$ & 6 & 3 & 3 & 3 & 2 & 1 & 1 & $\mathrm{x}$ & - & - & - & $\mathrm{x}$ & - & $\mathrm{x}$ & $\mathrm{x}$ & $\mathrm{x}$ & $\mathrm{HyCh}$ \\
\hline 11148900 & CA_NaciBryson & 4 & 2 & 1 & 1 & - & 1 & 1 & $\mathrm{x}$ & Gage & - & - & $\mathrm{x}$ & - & $\mathrm{x}$ & $\mathrm{x}$ & $\mathrm{x}$ & Нy \\
\hline 383321122302101 & CA_NapaBale & 6 & 3 & 3 & 3 & 2 & 1 & 1 & $\mathrm{x}$ & PT & $\mathrm{x}$ & $\mathrm{x}$ & $\mathrm{x}$ & - & $\mathrm{x}$ & $\mathrm{x}$ & $\mathrm{x}$ & $\mathrm{HyCh}$ \\
\hline 11458300 & CA NapaNapa & 6 & 3 & 3 & 3 & 2 & 1 & 1 & $\mathrm{x}$ & PT & $\mathrm{x}$ & - & $\mathrm{x}$ & - & $\mathrm{x}$ & $\mathrm{x}$ & $\mathrm{x}$ & $\mathrm{HyCh}$ \\
\hline 364155121363901 & CA_Natividad & 6 & 3 & 3 & 3 & 2 & 1 & 1 & $\mathrm{x}$ & PT & $\mathrm{x}$ & - & $\mathrm{x}$ & - & $\mathrm{x}$ & $\mathrm{x}$ & $\mathrm{x}$ & Нy \\
\hline 382346122521201 & $\begin{array}{l}\mathrm{CA}_{-} \\
\text {NoNameMonty }\end{array}$ & 6 & 3 & 3 & 3 & 2 & 1 & 1 & $\mathrm{x}$ & PT & $\mathrm{x}$ & - & $\mathrm{x}$ & - & $\mathrm{x}$ & $\mathrm{x}$ & $\mathrm{x}$ & Нy \\
\hline 365718121444301 & CA_NoNamePaul & 6 & 3 & 3 & 3 & 2 & 1 & 3 & - & PT & $\mathrm{x}$ & - & - & - & $\mathrm{x}$ & $\mathrm{x}$ & $\mathrm{x}$ & Ну \\
\hline 11459500 & CA_Novato & 6 & 3 & 3 & 3 & 2 & 1 & 1 & $\mathrm{x}$ & Gage & $\mathrm{x}$ & - & $\mathrm{x}$ & - & $\mathrm{x}$ & $\mathrm{x}$ & $\mathrm{x}$ & $\mathrm{HyCh}$ \\
\hline 11141050 & CA_Orcutt & 6 & 3 & 3 & 3 & 2 & 1 & 1 & - & Gage & $\mathrm{x}$ & - & - & - & $\mathrm{x}$ & $\mathrm{x}$ & $\mathrm{x}$ & Hy \\
\hline 365736121250801 & CA_Pacheco & 6 & 3 & 3 & 3 & 2 & 1 & 3 & - & PT & $\mathrm{x}$ & - & $\mathrm{x}$ & - & $\mathrm{x}$ & $\mathrm{x}$ & $\mathrm{x}$ & Нy \\
\hline 11159000 & CA_PajaroChit & 6 & 3 & 3 & 3 & 2 & 1 & 3 & - & Gage & $\mathrm{x}$ & - & - & - & $\mathrm{x}$ & $\mathrm{x}$ & $\mathrm{x}$ & Hy \\
\hline 11159500 & $\mathrm{CA}^{-}$PajaroWat & 6 & 3 & 3 & 3 & 2 & 1 & 3 & $\mathrm{x}$ & - & - & - & - & - & $\mathrm{x}$ & $\mathrm{x}$ & $\mathrm{x}$ & Нy \\
\hline 381519122385601 & CA_Petaluma & 6 & 3 & 3 & 3 & 2 & 1 & 1 & $\mathrm{x}$ & PT & $\mathrm{x}$ & - & - & - & - & - & - & - \\
\hline 375807122124001 & CA_Pinole & 4 & 2 & 1 & 1 & - & 1 & 1 & - & - & - & - & - & - & $\mathrm{x}$ & $\mathrm{x}$ & $\mathrm{x}$ & $\mathrm{HyCh}$ \\
\hline 11152650 & $\mathrm{CA}^{-}$Reclamation & 6 & 3 & 3 & 3 & 2 & 1 & 1 & $\mathrm{x}$ & Gage & $\mathrm{x}$ & - & $\mathrm{x}$ & - & $\mathrm{x}$ & $\mathrm{x}$ & $\mathrm{Tx}$ & Hy \\
\hline 383305122311901 & CA_Ritchey & 4 & 2 & 1 & 1 & - & 1 & 1 & $\mathrm{x}$ & PT & $\mathrm{x}$ & - & $\mathrm{x}$ & - & $\mathrm{x}$ & $\mathrm{x}$ & $\mathrm{x}$ & Hy \\
\hline 11150500 & CA_Salinas & 6 & 3 & 3 & 3 & 2 & 1 & 1 & - & Gage & $\mathrm{x}$ & - & - & - & $\mathrm{x}$ & $\mathrm{x}$ & $\mathrm{x}$ & Hy \\
\hline 372716122080801 & $\mathrm{CA}^{-}$SanFranPalo & 6 & 3 & 3 & 3 & 2 & 1 & 1 & - & - & - & - & - & - & $\mathrm{x}$ & $\mathrm{x}$ & $\mathrm{x}$ & $\mathrm{HyCh}$ \\
\hline 11164500 & CA_SanFranStan & 6 & 3 & 3 & 3 & 2 & 1 & 1 & $\mathrm{x}$ & Gage & $\mathrm{x}$ & - & - & - & $\mathrm{x}$ & $\mathrm{x}$ & $\mathrm{x}$ & $\mathrm{HyCh}$ \\
\hline 374336122095801 & CA_SanLeandro & 6 & 3 & 3 & 3 & 2 & 1 & 1 & $\mathrm{x}$ & - & - & - & - & - & $\mathrm{x}$ & $\mathrm{x}$ & $\mathrm{x}$ & $\mathrm{HyCh}$ \\
\hline 351436120405201 & CA SanLObispo & 6 & 3 & 3 & 3 & 2 & 1 & 1 & - & PT & $\mathrm{x}$ & - & $\mathrm{x}$ & - & $\mathrm{x}$ & $\mathrm{x}$ & $\mathrm{x}$ & Hy \\
\hline 11181000 & CA_SanLorenzo & 6 & 3 & 3 & 3 & 2 & 1 & 1 & - & Gage & - & - & - & - & $\mathrm{x}$ & $\mathrm{x}$ & $\mathrm{x}$ & $\mathrm{HyCh}$ \\
\hline 375220122104201 & CA_SanPabloMor & 6 & 3 & 3 & 3 & 2 & 1 & 1 & - & - & - & - & $\mathrm{x}$ & - & $\mathrm{x}$ & $\mathrm{x}$ & $\mathrm{x}$ & $\mathrm{HyCh}$ \\
\hline 375312122113501 & CA_SanPabloOr & 6 & 3 & 3 & 3 & 2 & 1 & 1 & - & - & - & - & $\mathrm{x}$ & $\mathrm{x}$ & $\mathrm{x}$ & $\mathrm{x}$ & $\mathrm{x}$ & $\mathrm{HyCh}$ \\
\hline 375746122195501 & CA_SanPabloPort & 6 & 3 & 3 & 3 & 2 & 1 & 1 & $\mathrm{x}$ & - & - & - & - & - & $\mathrm{x}$ & $\mathrm{x}$ & $\mathrm{x}$ & $\mathrm{HyCh}$ \\
\hline 374933122001301 & CA_SanRamonDan & 6 & 3 & 3 & 3 & 2 & 1 & 1 & $\mathrm{x}$ & - & - & - & $\mathrm{x}$ & - & $\mathrm{x}$ & $\mathrm{x}$ & $\mathrm{x}$ & $\mathrm{HyCh}$ \\
\hline 11182500 & CA SanRamonSan & 6 & 3 & 3 & 3 & 2 & 1 & 1 & $\mathrm{x}$ & Gage & - & - & $\mathrm{x}$ & - & $\mathrm{x}$ & $\mathrm{x}$ & $\mathrm{x}$ & $\mathrm{HyCh}$ \\
\hline 11466200 & CA_SantaSanta & 6 & 3 & 3 & 3 & 2 & 1 & 1 & $\mathrm{x}$ & Gage & $\mathrm{x}$ & - & $\mathrm{x}$ & - & $\mathrm{x}$ & $\mathrm{x}$ & $\mathrm{x}$ & $\mathrm{HyCh}$ \\
\hline 11466320 & CA_SantaWillow & 6 & 3 & 3 & 3 & 2 & 1 & 1 & $\mathrm{x}$ & Gage & $\mathrm{x}$ & - & $\mathrm{x}$ & - & $\mathrm{x}$ & $\mathrm{x}$ & Tx & $\mathrm{HyCh}$ \\
\hline
\end{tabular}


Table 5. Summary of data collected at the California Stream Quality Assessment sites in 2017.-Continued

[Constituents are categorized by the study-design components: Comprehensive Stressor Assessments, Focused Studies, and Ecological Surveys. Notes and abbreviations: See footnote 1 and text for Basic quality of water (QW) constituents. Abbreviations: C, continuous sample; D, discrete samples; I, integrative sample. Numerical value in cell indicates number of discrete samples collected: x, data or sample collected; - , not sampled. Streamflow or stage: Egage, gaging station for an external agency other than U.S. Geological Survey (USGS); Gage, USGS gaging station; PT, pressure transducer installed to record stream stage. Ecological Surveys were conducted once at sites near the end of the water-quality index period: Aquatic Biota, invertebrate and algal samples collected; Streambed

Sediment: Tx, two temporal samples were collected prior to collecting sediment during ecological survey; Sediment Toxicity Test, sediment tested on survival of invertebrates: Ch, Chironomus larvae; Hy, Hyalella. Other abbreviations: N, nitrogen; NWIS; U.S. Geological Survey National Water Information System database; O, oxygen; POCIS, polar organic chemical integrative sampler; SS, suspended sediment]

\begin{tabular}{|c|c|c|c|c|c|c|c|c|c|c|c|c|c|c|c|c|c|c|}
\hline \multicolumn{2}{|c|}{ Site information } & \multicolumn{10}{|c|}{ Comprehensive stressor assessments } & \multicolumn{3}{|c|}{ Focused studies } & \multicolumn{4}{|c|}{ Ecological surveys } \\
\hline $\begin{array}{l}\text { NWIS station } \\
\text { number }\end{array}$ & Field identifier & $\begin{array}{c}\text { Basic } \\
\text { OW1 } \\
\text { (D) }\end{array}$ & $\begin{array}{l}\text { Mer- } \\
\text { cury } \\
\text { (D) }\end{array}$ & $\begin{array}{c}\text { Organic } \\
\text { waste } \\
\text { indica- } \\
\text { tors } \\
\text { (D) }\end{array}$ & $\begin{array}{l}\text { Phar- } \\
\text { ma- } \\
\text { ceuti- } \\
\text { cals } \\
\text { (D) }\end{array}$ & $\begin{array}{l}\text { Glypho- } \\
\text { sate } \\
\text { analysis } \\
\text { (D) }\end{array}$ & $\begin{array}{l}\mathrm{N} \text { and } \mathbf{0} \\
\text { iso- } \\
\text { topes } \\
\text { (D) }\end{array}$ & $\begin{array}{l}\text { Algal } \\
\text { toxins } \\
\text { (D) }\end{array}$ & $\begin{array}{l}\text { POCIS } \\
\left(\text { I) }{ }^{2}\right.\end{array}$ & $\begin{array}{l}\text { Stream- } \\
\text { flow or } \\
\text { stage } \\
\text { (C) }{ }^{3}\end{array}$ & $\begin{array}{l}\text { Tem- } \\
\text { pera- } \\
\text { ture } \\
\text { (C) }\end{array}$ & $\begin{array}{c}\text { Pes- } \\
\text { ticide } \\
\text { auto- } \\
\text { sampler } \\
\text { (I) }\end{array}$ & $\begin{array}{l}\text { Bio- } \\
\text { film } \\
\text { pesti- } \\
\text { cides } \\
\text { (I) }\end{array}$ & $\begin{array}{c}\text { SS } \\
\text { passive } \\
\text { sam- } \\
\text { pler } \\
\text { (I) }\end{array}$ & $\begin{array}{l}\text { Aquat- } \\
\text { ic biota } \\
\text { (D) }\end{array}$ & $\begin{array}{l}\text { Physi- } \\
\text { cal } \\
\text { habitat } \\
\text { (D) }\end{array}$ & $\begin{array}{l}\text { Stream- } \\
\text { bed } \\
\text { sedi- } \\
\text { ment } \\
\text { (D) }\end{array}$ & $\begin{array}{c}\text { Sedi- } \\
\text { ment } \\
\text { toxicity } \\
\text { test } \\
\text { (D) }\end{array}$ \\
\hline 11135250 & $\begin{array}{l}\mathrm{CA}_{-} \\
\text {SantaYnezLom }\end{array}$ & 6 & 3 & 3 & 3 & 2 & 1 & 1 & $\mathrm{x}$ & - & $\mathrm{x}$ & - & $\mathrm{x}$ & - & $\mathrm{x}$ & $\mathrm{x}$ & $\mathrm{x}$ & Нy \\
\hline 11128500 & CA_SantaYnezSol & 6 & 3 & 3 & 3 & 2 & 1 & 1 & $\mathrm{x}$ & Gage & $\mathrm{x}$ & - & - & - & $\mathrm{x}$ & $\mathrm{x}$ & $\mathrm{x}$ & Нy \\
\hline 371620122005801 & CA_Saratoga & 6 & 3 & 3 & 3 & 2 & 1 & 1 & $\mathrm{x}$ & - & - & - & $\mathrm{x}$ & - & $\mathrm{x}$ & $\mathrm{x}$ & $\mathrm{x}$ & Нy \\
\hline 374708122132801 & CA_Sausal & 6 & 3 & 3 & 3 & 2 & 1 & 1 & $\mathrm{x}$ & - & - & - & $\mathrm{x}$ & - & $\mathrm{x}$ & $\mathrm{x}$ & $\mathrm{x}$ & $\mathrm{HyCh}$ \\
\hline 382634122315201 & CA_SonomaAdobe & 4 & 2 & 1 & 1 & - & 1 & 1 & $\mathrm{x}$ & - & - & - & $\mathrm{x}$ & - & $\mathrm{x}$ & $\mathrm{x}$ & $\mathrm{x}$ & $\mathrm{HyCh}$ \\
\hline 11458500 & CA_SonomaAgua & 6 & 3 & 3 & 3 & 2 & 1 & 1 & $\mathrm{x}$ & Gage & $\mathrm{x}$ & - & - & - & $\mathrm{x}$ & $\mathrm{x}$ & $\mathrm{x}$ & Hy \\
\hline 381556122280201 & CA_SonomaWat & 6 & 3 & 3 & 3 & 2 & 1 & 1 & $\mathrm{x}$ & PT & $\mathrm{x}$ & - & - & - & $\mathrm{x}$ & $\mathrm{x}$ & $\mathrm{x}$ & Нy \\
\hline 351725120395901 & CA_Stenner & 6 & 3 & 3 & 3 & 2 & 1 & 1 & $\mathrm{x}$ & PT & $\mathrm{x}$ & - & - & - & $\mathrm{x}$ & $\mathrm{x}$ & $\mathrm{x}$ & Hy \\
\hline 381441122064301 & CA_Suisan & 6 & 3 & 3 & 3 & 2 & 1 & 1 & $\mathrm{x}$ & Egage & - & - & $\mathrm{x}$ & $\mathrm{x}$ & $\mathrm{x}$ & $\mathrm{x}$ & $\mathrm{x}$ & Нy \\
\hline 365634121264001 & CA_Tequisquita & 6 & 3 & 3 & 3 & 2 & 1 & 3 & $\mathrm{x}$ & PT & $\mathrm{x}$ & - & $\mathrm{x}$ & - & $\mathrm{x}$ & $\mathrm{x}$ & $\mathrm{x}$ & Hy \\
\hline 382245122001601 & CA_Ulatis & 6 & 3 & 3 & 3 & 2 & 1 & 1 & $\mathrm{x}$ & Egage & - & - & $\mathrm{x}$ & $\mathrm{x}$ & $\mathrm{x}$ & $\mathrm{x}$ & $\mathrm{x}$ & Hy \\
\hline 365955121350601 & CA_UvasMiller & 6 & 3 & 3 & 3 & 2 & 1 & 1 & - & - & $\mathrm{x}$ & - & $\mathrm{x}$ & - & $\mathrm{x}$ & $\mathrm{x}$ & $\mathrm{x}$ & Hy \\
\hline 375413122033301 & CA_Walnut & 6 & 3 & 3 & 3 & 2 & 1 & 1 & - & Egage & - & - & $\mathrm{x}$ & - & $\mathrm{x}$ & $\mathrm{x}$ & $\mathrm{x}$ & $\mathrm{HyCh}$ \\
\hline 375808122172601 & CA_Wilkie & 6 & 3 & 3 & 3 & 2 & 1 & 1 & $\mathrm{x}$ & - & - & - & $\mathrm{x}$ & - & $\mathrm{x}$ & $\mathrm{x}$ & $\mathrm{x}$ & $\mathrm{HyCh}$ \\
\hline 383039122502401 & CA_Windsor & 6 & 3 & 3 & 3 & 2 & 1 & 1 & - & PT & $\mathrm{x}$ & - & $\mathrm{x}$ & - & $\mathrm{x}$ & $\mathrm{x}$ & $\mathrm{x}$ & $\mathrm{HyCh}$ \\
\hline
\end{tabular}

${ }_{1}^{1 B a s i c}$ QW constituents: suspended sediment, major ions, nutrients, pesticides, supplemental pesticides, glyphosate immunoassay, and dissolved organic carbon.

2Data at some sites were missing because sample or instrument was lost.

${ }^{3}$ Continuous stage or temperature data were missing at some sites because pressure or temperature sensors were either not deployed or lost in the field owing to high flow conditions. 


\section{Discrete Water-Quality Samples}

During the WQ index period of each site, Basic QW samples were collected weekly for analysis of nutrients, major ions, dissolved organic carbon, pesticides (using an expanded target analyte list relative to prior RSQA studies), glyphosate by immunoassay, and suspended-sediment concentration (table 5; appendix 1, table 1.1). Additional water samples were collected or parameters were measured during selected weeks during the WQ index period at all 85 stream sites, unless specified otherwise, for the following: mercury (2-3 times during the study), organic waste indicators (1-3 times), pharmaceuticals ( $1-3$ times), glyphosate by chemical analysis ( 2 times at 83 sites), stable isotopes ${ }^{15} \mathrm{~N}$ and ${ }^{18} \mathrm{O}$ (1 time), and algal toxins ( 1 time at 77 sites, 3 times at 8 sites, plus 2 times at 1 nearby lake site). The weeks of the WQ index period during which these additional samples were collected or parameters were measured are specified in appendix 1, table 1.1. For algal toxins, one water sample was collected during the last week of the study period at all stream sites. In addition, two water samples were collected on July 11 and 25, 2017, at eight stream sites in the Pajaro River Basin and at one site in Pinto Lake (into which the Pajaro River flows), because of a history of microcystin contamination there (California Water Boards, 2014; Gibble and Kudela, 2014).

Water-quality samples were collected and processed following standard USGS protocols described in the National Field Manual (Wilde and others, 2009). Before collecting samples from the field, all field equipment was cleaned according to USGS protocols and then rinsed with native water immediately before samples were collected. In general, discrete water samples were collected for most analytes by an isokinetic, equal-width increment method (U.S. Geological Survey, 2006; fig. 5) where subsamples were collected at 10 increments across the stream with either a DH-81 or DH-95 sampler (Davis, 2005). The sampler has a precleaned Teflon cap and nozzle assembly that fitted a 1-liter (L) Teflon bottle (U.S. Geological Survey, 2006). Each incremental sample was placed immediately into a precleaned, acid- and methanol-rinsed Teflon churn for compositing before processing.

Water from the Teflon churn was not used for mercury analysis because ultra-trace concentration clean-sampling procedures and equipment were necessary to collect and process the samples for low-concentration total mercury and methylmercury analysis (U.S. Environmental Protection Agency, 1996; Lewis and Brigham, 2004). Water for mercury samples was collected separately as grab samples from the centroid of flow at about $0.3 \mathrm{~m}$ below the water surface in a 1-L Nalgene bottle. These samples were collected three times at the 6-week sites and two times at the 4-week sites (table 5, appendix 1, table 1.1). In addition, the dissolved organic carbon (DOC) samples were collected as grab samples from the centroid of flow; because mercury bioavailability is related to DOC levels in the aquatic environment, collecting mercury and DOC samples using the same method was deemed important to establish a relation between the two constituents. Filtering of samples utilized low-level mercury sampling techniques as described in the USGS Field Manual (Wilde and others, 2009, Section 5.6.4.B). After filtering, samples were preserved using ultrapure hydrochloric acid (Hydrochloric Acid 34-37 percent OmniTrace ${ }^{\circledR}$ ), and filters were frozen (Wilde and others, 2009). Samples were processed at either a USGS California Laboratory or at the USGS Mercury Research Laboratory in Middleton, Wisconsin. Samples processed in California were shipped to the USGS Mercury Research Laboratory for mercury analysis.

A separate water sample was collected at all sites to determine concentrations of the algal toxin microcystin in streams (table 5; appendix 1, table 1.1) and at one lake site. These water samples were frozen $\left(-20^{\circ} \mathrm{C}\right)$ and shipped overnight to the USGS Organic Geochemistry Research Laboratory (OGRL) in Lawrence, Kansas, for processing and analysis.

When stream conditions did not meet the requirements for collecting equal-width increment subsamples with the Teflon churn (which required a velocity greater than 1.5 feet per second $[\mathrm{ft} / \mathrm{s}]$ ), samples were collected either by a multivertical grab (for velocity less than $1.5 \mathrm{ft} / \mathrm{s}$, width greater than 10 feet [ft]), or by a grab from the centroid of flow (for velocity less than $1.5 \mathrm{ft} / \mathrm{s}$, width less than 10 feet, depth less than 1 foot). When flow conditions were too high to safely collect equal-width increment subsamples, a single-point grab sample was collected from the bank or by wading as far as possible into the stream. For grab samples, water was collected directly into sample bottles for unfiltered constituents and into a precleaned 1-L Teflon sample bottle for filtered constituents; samples were subsequently filtered from that bottle into sample bottles (appendix 1, table 1.3).

California had a relatively wet year in 2017 , particularly during the month of February, which preceded the study period. Overall, monthly runoff in California was higher during the March 3-May 13, 2017, study period than the average value over the previous 30 water years (a water year is the period beginning October 1st of one year and ending September 30th of the next year; https://ca.water.usgs.gov/ california-drought/california-drought-runoff.html). High flow conditions occurred in some streams, in which case grab samples had to be collected (as described earlier in the text). At three Northern sites, high flow conditions at the end of the study period prevented collection of ecological samples, so ecological survey data were available for only 82 sites (appendix 1, table 1.1).

Field properties of specific conductance, $\mathrm{pH}$, dissolved oxygen, and water temperature were measured at the time of each weekly water-quality sampling with a field-calibrated multiparameter sonde, as described in the USGS National Field Manual (Wilde, 2008). The measurements were made at five locations within the water-quality sampling transect and averaged. When the stream width was less than $10 \mathrm{ft}(3 \mathrm{~m})$, field parameters were measured from the centroid of flow. 


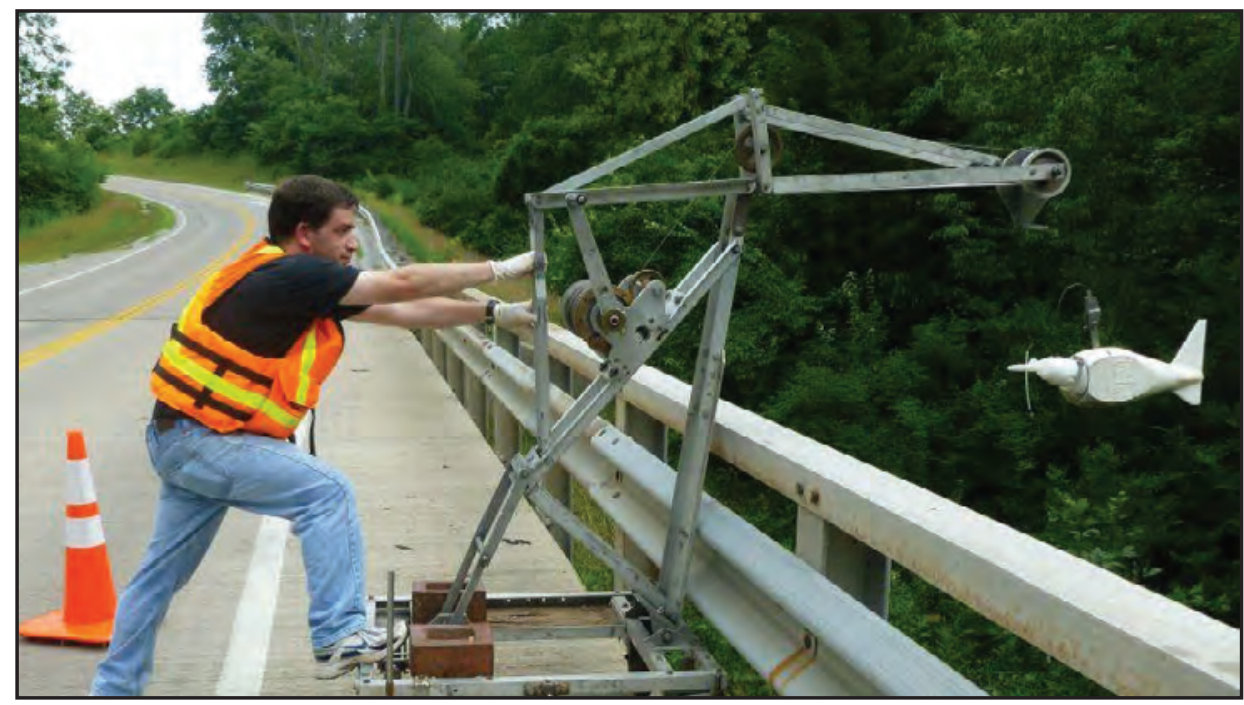

Figure 5. A hydrologist moves an isokinetic sampler into position. Photograph by Stephanie Kula, U.S. Geological Survey.

\section{Polar Organic Compound Integrative Samplers (POCIS)}

Two POCIS housed within a single canister were deployed for between 33 and 38 days (about 5 weeks) at each of the 85 sites during the WQ index period; one POCIS from each canister was analyzed for pesticides and one for pharmaceuticals. Canisters were lost or compromised (such as by burial in sediment or exposure to air) at 21 sites, leaving 64 sites with valid POCIS samples (table 5; appendix 1, table 1.1). The POCIS are integrative samplers that contain the sorbent Oasis HLB (Waters, Milford, Mass.) and are designed to accumulate moderately water-soluble (polar or hydrophilic) organic compounds from surface water (Alvarez, 2010). Oasis HLB is considered a universal sorbent in environmental analyses and has been used to extract a wide assortment of chemical classes from water. The use of Oasis HLB in the POCIS allows for estimation of time-weighted average concentrations of target chemicals (pesticides and pharmaceuticals) over the deployment period, if a chemical-specific water "sampling rate" has been determined. Extracts of POCIS samples were analyzed for contaminants by using modified versions of the water methods for these chemical groups (Van Metre and others, 2017a).

Field deployment followed the guidelines provided in Alvarez (2010). A stream location was required to have enough depth (about $0.5 \mathrm{ft}$, or 15 centimeters [cm]) for the POCIS to remain submerged during the deployment period and be protected from excessive sediment accumulation, flood debris, and vandalism. Effective anchoring systems were adopted on the basis of site-specific characteristics (for example, sandy versus rocky substrate, streamflow variability, and so forth). The POCIS canister was attached to the same structure as the temperature data logger at many sites (see section "Continuous Streamflow, Stage, and Water Temperature"), either on the rebar or on the streamgage orifice pipe. Others were deployed in the centroid of flow and attached to a cinder block (fig. 6). Field records were maintained that included the site name; date and time of deployment and retrieval; and observations of streambed substrate, streamflow conditions, and water clarity.

Quality control (QC) POCIS samples collected in the field included replicates and blanks and are described in the "Quality Assurance and Quality Control" section. All environmental POCIS and QC canisters were stored on ice during transport to and from the field location. After the deployment period, the POCIS were retrieved from the sites and immediately sealed in their respective canisters; all environmental POCIS, QC canisters, and log sheets were shipped to the USGS Columbia Environmental Research Center (CERC), Columbia, Missouri, in coolers with wet ice.

\section{Continuous Streamflow, Stage, and Water Temperature}

The sampling design called for measurement of streamflow or stage at all sites, as well as continuous temperature. However, stage or temperature data were missing at some sites because pressure or temperature sensors were either not deployed or lost in the field due to high flow conditions. Overall, streamflow or stage was obtained at 54 sites and continuous temperature at 46 sites; details are provided in table 5 . 


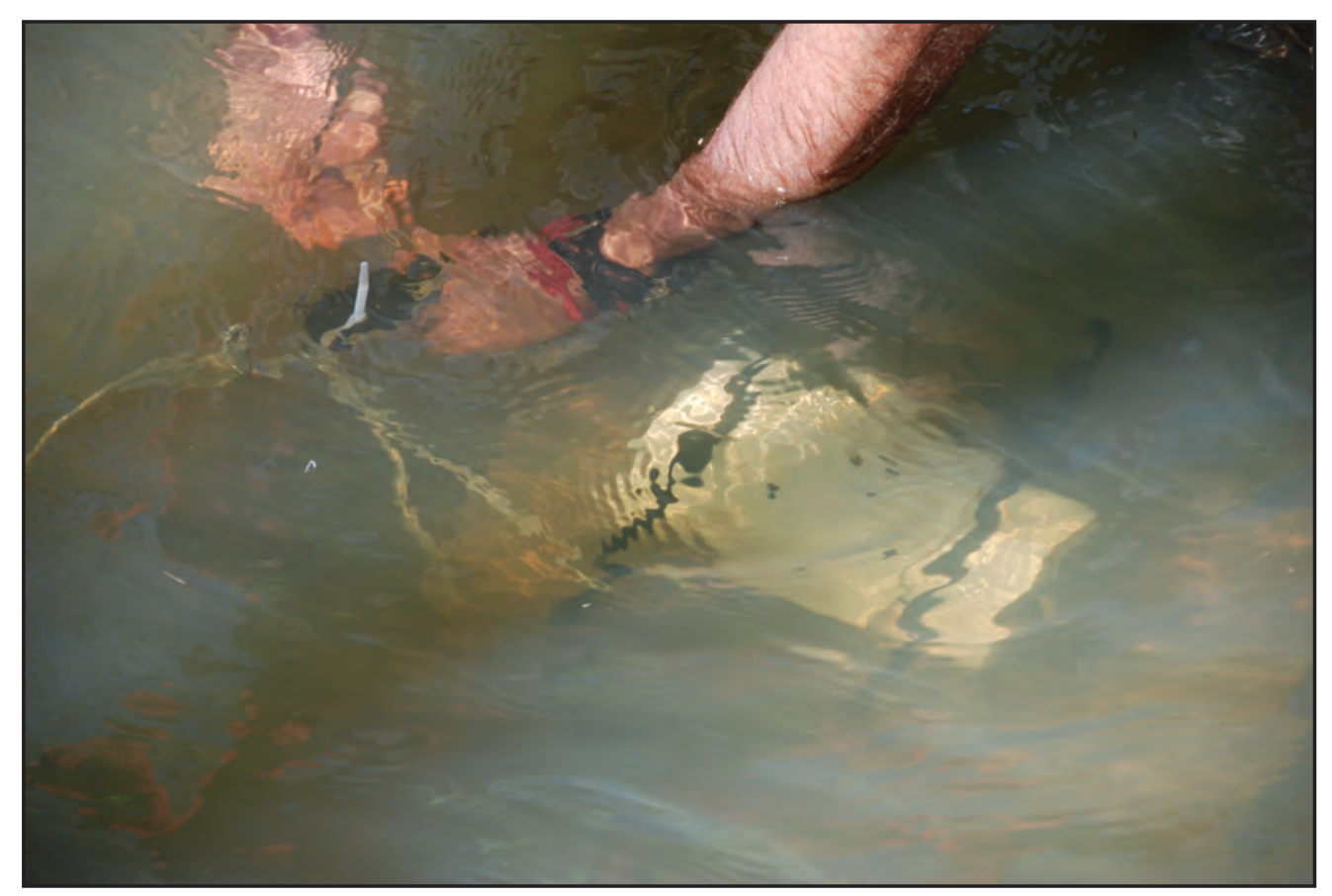

Figure 6. Deployed polar organic compound integrated sampler on cinder-block-and-cable infrastructure. Photograph by Celeste Journey, U.S. Geological Survey.

U.S. Geological Survey streamgages were active at 28 sites and provided stream stage and streamflow discharge at 15-minute intervals (table 5). Four sites had continuous stage data from streamgages operated by county or city agencies. Pairs of pressure transducers (one in the water column and one in the air, as described farther on in the text) were successfully deployed at 19 sites, and these units were programmed to record stream stage at 15 -minute intervals. Neither flow nor stage was monitored at the remaining 34 sites. The pressure transducers were the HOBO U20-001-04 digital loggers (specifications are in appendix 1, table 1.2). The units were deployed during February 2017, before the start of the WQ index periods, and were retrieved in the fall of 2017. Guidance from the manufacturer and the USGS was followed for deployment, calibration, and maintenance of the units (Sauer and Turnipseed, 2010; Onset Computer Corporation, 2014).

Full deployment of pressure transducers at a site included the installation of two units per site: one unit mounted in the water column to measure changes in water pressure as the water level changed, and one unit mounted in the air to measure barometric pressure to provide a correction factor for calculating stream stage with the water-pressure transducer data. The units were placed inside a vertical 2 -inch polyvinyl chloride pipe mounted to a bridge support or directly to a metal post driven into the streambed. The water-pressure units were mounted at a depth where they would remain continually submerged, and the barometric-pressure units were typically mounted to a tree, bridge structure, pipe, or post. Both units were programmed to record on 15-minute intervals for the duration of the study.

Establishing a baseline water-level was necessary immediately after deployment of the pressure transducer in the water so that measurements of water pressure could be converted to actual water level. A reference point (RP), on which changes in water level were based, was established upstream from the pool that held the submerged unit.

Typically, the RP was a mark scribed on a permanently fixed structure near the attached unit, such as a bridge support or wing wall; a measurement from the RP to the water surface was the "tape down" distance. An arbitrary datum was then established that was greater than the distance between the RP and the channel bottom, and this datum would cover all low stages and ensure no negative stage values; typically, 10 feet was used as the water level at the streamgage datum. The distance from the RP to the surface of the water at time of deployment was used to establish the initial stream stage. In addition, measurements from the RP to water surface were made during every site visit by the water-quality sampling crew, so that these values could be used to check data for consistency and quality. 
Water temperature was continuously monitored at 2 sites with streamgages, 23 sites with instream pressure transducers, and 21 sites with digital temperature data loggers; continuous temperature was not recorded at the remaining 39 sites. The pressure transducers included temperature sensors that were used to record water temperature at 15-minute intervals. Deployment of a single pressure transducer mounted in the water column was sufficient to measure continuous temperature. Although pressure transducers were successfully deployed to measure stage at only 19 sites, instream pressure transducers recorded temperature at an additional 4 sites. At some stream sites that did not have a streamgage or pressure transducer recording temperature, digital data loggers recorded temperature at 15-minute intervals. These devices were deployed from early winter 2017 through the end of the study in order to provide a water-temperature dataset inclusive of all sampling activities. Where possible, loggers were deployed approximately $0.3 \mathrm{ft}(10 \mathrm{~cm})$ above the streambed, out of direct sunlight, and attached to rebar anchored into the streambed or to stable parts of streamgage infrastructure (for example, orifice pipe). In most cases, the HOBO Water Temp Pro v2 U22 loggers were deployed (device specifications are in appendix 1, table 1.2). Guidance from the manufacturer and the U.S. Forest Service concerning deployment, calibration, and maintenance generally was followed (Dunham and others, 2005; Onset Computer Corporation, 2012).

\section{Focused Studies}

Three types of focused studies were conducted at selected sites (table 5). Small-volume autonomous water samplers were deployed at five sites to collect daily composited water samples for pesticide analysis. Ceramic tiles were deployed at 54 sites to provide a substrate for the growth of biofilms that were collected and analyzed for selected pesticides. Passive suspended-sediment samplers were deployed at five sites to collect time-integrated samples of suspended sediment.

\section{Sampling Pesticides with Small-Volume Pesticide Automated Samplers}

A small-volume autonomous water autosampler (hereafter, "pesticide autosampler") was designed and built at Portland State University, Oregon, to collect fixed-point, small-volume samples (McWhirter, 2020) for analysis of pesticides in water. In a collaborative study with EPA, the autosamplers were used at a subset of five CSQA sites to determine whether increasing sampling frequency would improve the accuracy of characterizations of instream pesticide stressor conditions to which biota were subjected.
Although weekly discrete samples of pesticides were collected at all sites during the WQ index periods, discrete samples can miss short-term "spikes" in high concentrations that are potentially acutely toxic. The pesticide autosamplers were deployed at five Northern stream sites to collect daily and weekly composite samples over an approximately 6-week WQ index period: two high urban sites, one medium urban site, one high agriculture site, and one low agriculture site, as classified by lower-basin scale land use (tables 1 and 5 , cross referenced). The autosampler was discontinued at one high urban site owing to repeated flooding. As part of this collaborative study with EPA, autosamplers also were deployed at two agricultural sites in the Central Valley (outside the CSQA study area), Cache Creek and Putah Creek.

The pesticide autosamplers (fig. 7) were programmed to collect multiple aliquots to form daily and weekly composite samples of stream water during successive 1-week periods. An aliquot of stream water was collected every 6 hours into daily composite vials (four aliquots per vial, with the "day" typically starting around noon) and every 12 hours into the weekly composite vial. Thus, eight vials were filled per week for 7 daily samples and 1 weekly sample. In addition, a ninth vial containing a known pesticide spike mixture in native stream water was placed in the autosampler at the start of the week; this QC sample was used to assess the potential for compound degradation during the weekly collection period. A 6-milliliter $(\mathrm{mL})$ aliquot of a 1:1 methanol-water mixture was added, as a preservative, to each of the nine vials before deployment.

Over the 6 weeks of operation, the pesticide autosamplers were serviced each week on either Monday or Tuesday. Two units were available for each site, so that one unit could be serviced in the laboratory and exchanged in the field for the deployed unit. This arrangement minimized interruption by allowing ample time to remove and replace sample vials, charge batteries, clean tubing, and replace consumable components such as filters. Before deployment, each vial was labeled with the station identification number, vial number, date, and initial weight. Daily composite samples (vials 1 through 7) were analyzed for pesticide concentrations by the EPA Office of Pesticide Programs (OPP) Analytical Chemistry Laboratory in Fort Meade, Maryland. Sample splits of the weekly composite sample (vial 9) and the spike sample (vial 8) were analyzed for pesticides by both the USGS National Water Quality Laboratory (NWQL) in Denver, Colorado, and the OPP laboratory. Analytical service request (ASR) forms (USGS) and cooler inventory forms (USGS and EPA) were included with sample shipments, and barcodes were affixed to each vial as an auxiliary data identifier and tracking method. 

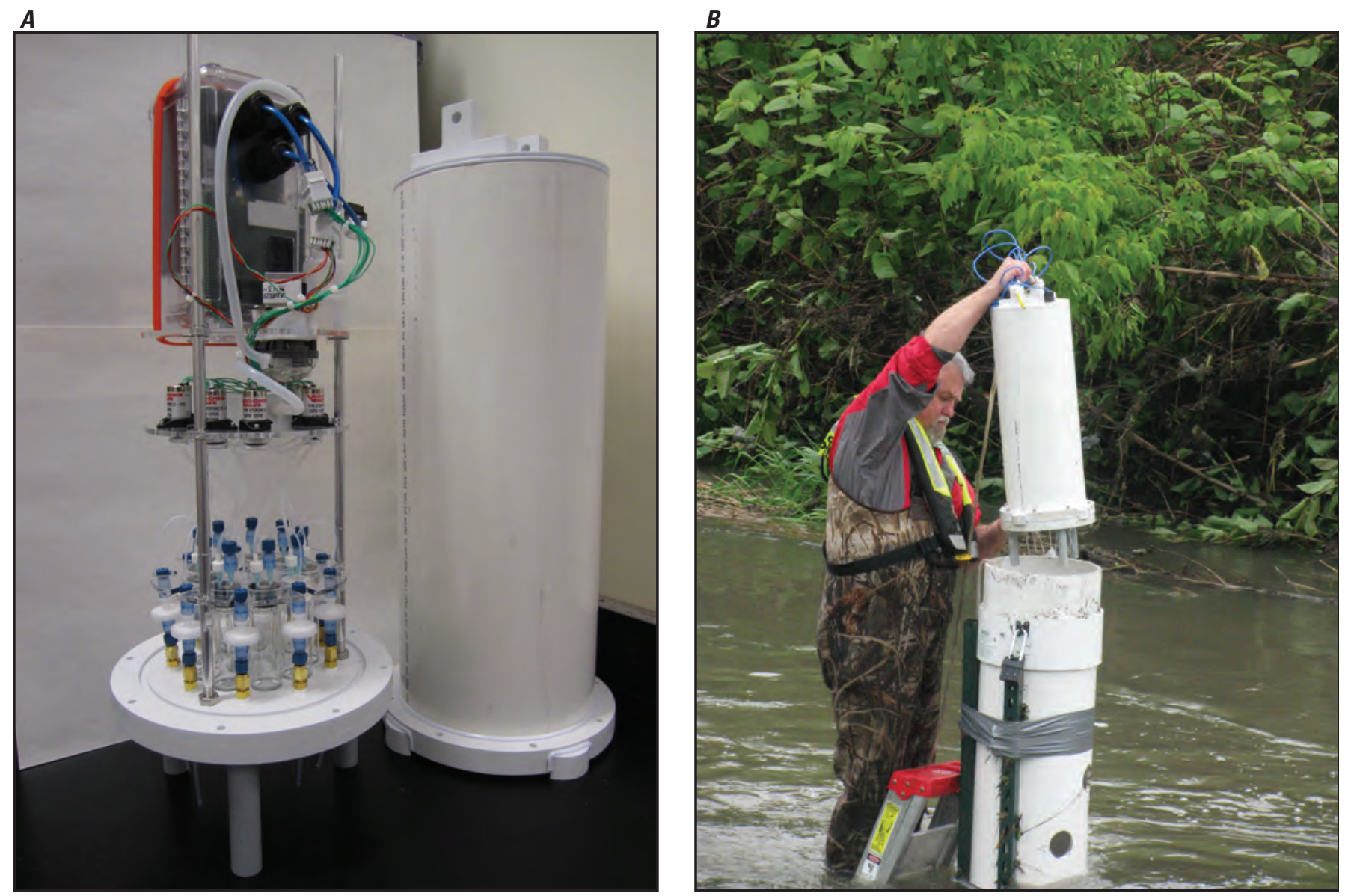

Figure 7. The small-volume pesticide autosampler used to collect filtered water at sub-daily intervals for pesticide analysis at selected sites: $A$, autosampler internals and the polyvinyl chloride housing (from McWhirter, 2020; photograph by Kevin McWhirter, Portland State University); and $B$, a hydrologic technician retrieving samples from the autosampler during deployment in the stream. Photograph by Peter Van Metre, U.S. Geological Survey. The samplers were designed and built at Portland State University (McWhirter, 2020).

\section{Sampling Pesticides in Biofilms}

A focused study was conducted in CSQA to determine whether pesticides bioaccumulate in biofilms (Barbara Mahler, written commun., 2020), which consist of fungi, bacteria, algae, and microfauna enclosed in a mucopolysaccharide matrix attached to a surface exposed to streamflow. This complex organic microlayer can retain organic contaminants, much like DOC (Sabater and others, 2007). Unglazed ceramic tiles were deployed at 54 sites that represented all land-use categories across the study area (tables 1 and 5, cross referenced) in February or March 2017. Tiles were secured to a concrete block about $30 \mathrm{~cm}$ thick using water-resistant cement, and three replicate tiles were distributed along the reach for a deployment period of at least 60 days (appendix 1, table 1.6). At each site, the biofilm that had grown on the tiles was removed by scraping and composited into a clean glass jar, placed in a cooler with ice, and delivered to the USGS Organic Chemistry Research Laboratory, Sacramento, Calif.
Samples were kept frozen at the laboratory until extraction and analysis were done for insecticides. Quality control samples consisted of one field replicate sample, four laboratory blank samples, and three laboratory matrix spikes.

\section{Sampling Suspended Sediment with Passive Samplers}

Suspended-sediment samples were collected from five Northern sites by using time-integrating suspended-sediment passive samplers (Phillips and others, 2000). The fine sediments (less than 63 micrometers $[\mu \mathrm{m}]$ ) were analyzed for major and trace elements, particle size, and radionuclides to assess sources and ages of sediment in the stream. These suspended-sediment passive samplers were deployed at three urban sites (one high, one medium, and one low urban), one agricultural site, and one mixed site, as characterized by lower-basin land use (tables 1 and 5, cross referenced). 
The suspended-sediment passive samplers were attached to metal posts that were driven into the streambed and were oriented so that the conical end (fig. 8) faced into the flow. Typically, four samplers were installed in the channel in two pairs; each pair consisted of a lower sampler placed below the water level at base flow and the paired sampler placed above the lower sampler. If base flows were especially low at the time of deployment, the top sampler was allowed to be out of the water; in such cases, the top samplers would collect sediment only at flows higher than base flow.

The suspended-sediment passive samplers were deployed on January 24 or 25, 2017, and remained deployed for approximately 16 weeks, through early May, inclusive of the WQ index period. Sediment was retrieved every 2-3 weeks or following a large flow event, allowing enough time for sufficient sediment to accumulate for analyses of major and trace elements and radionuclides. To collect the sediment samples, the tubes were removed from their posts, the end caps were opened, and all the water and sediment were poured into a single 5-gallon plastic bucket. A spray bottle filled with deionized water was used to rinse any remaining sediment from the tubes. After collecting the samples, the tubes were cleaned with a brush and deionized water, then rinsed with native water. The water-sediment mixture was stored at room temperature in the 5-gallon bucket until the sediment settled to the bottom (usually 3-7 days), then the clear water was siphoned off and discarded. The remaining composite sediment sample was shipped on wet ice to the USGS Baltimore Sediment Laboratory, Md., where it was wet sieved with deionized water using a $63-\mu \mathrm{m}$ polyester sieve. The sieved slurry was collected in glass bowls and dried at $65^{\circ} \mathrm{C}$ for 2 or more days. The dried sediment was removed with a plastic utensil, and aliquots of the fine sediment (less than $63 \mu \mathrm{m}$ ) were shipped to multiple laboratories (see "Sample Analyses" section) for inorganic chemical analyses. For about 10 percent of samples, if sufficient sediment mass was available, duplicate aliquots (QC split samples) were analyzed for major and trace elements, and particle size.

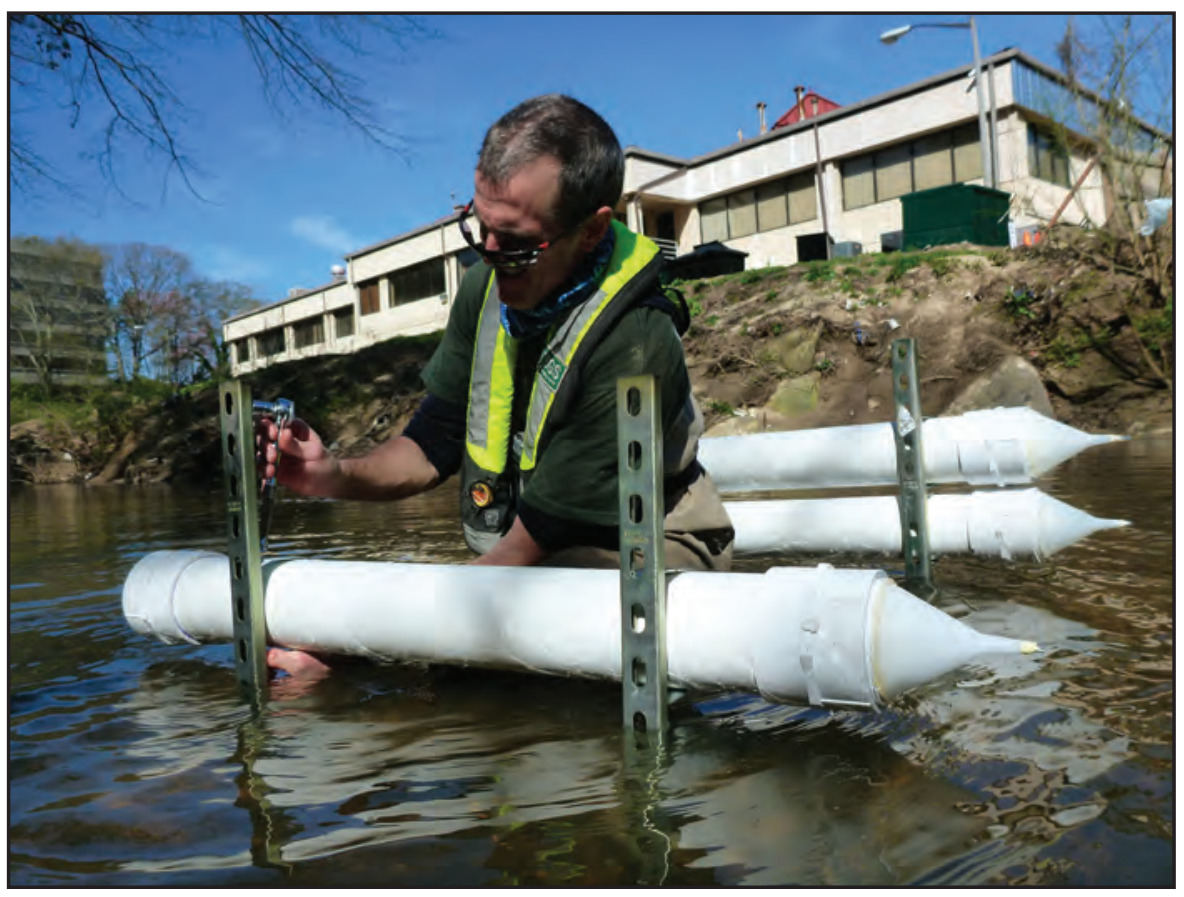

Figure 8. Installation of time-integrating suspended-sediment passive samplers by U.S. Geological Survey personnel. Photograph by Celeste Journey, U.S. Geological Survey. 


\section{Ecological Surveys}

Data collected during the ecological surveys characterize aquatic biota, physical habitat, and sediment contaminants along a $150-\mathrm{m}$ sampling reach for each site (table 5). Five teams, each consisting of six USGS employees, were deployed across the region to conduct the surveys at all sites during May 2017, after the end of the WQ index period. The median number of days between the end of water-quality sampling and the ecological survey was 3 days for the Northern sites and 18 days for the Southern sites. As noted previously, no surveys were conducted at three sites (table 5; appendix table 1.1) where high water prohibited safe sampling during the targeted time of the ecological survey. Although the data collected during the ecological surveys were based on discrete samples, biological and habitat data generally represent integrative conditions over a longer, undefined time period. For example, sediment chemistry is affected by erosional processes and contaminant persistence, the species structure of aquatic biological communities depends on water-quality conditions that occur over the life cycles of the organisms, and the physical habitat of a stream reach typically is strongly affected by many years of hydrologic events and human actions.

The 150-m sampling reach was segmented with 11 equally spaced cross-sectional transects (primary transects) that were set $15 \mathrm{~m}$ apart along the reach, and 10 secondary transects, each set approximately midway between a pair of primary transects. All field data were recorded on electronic forms by using hand-held tablet computers. Field data collected from the habitat surveys, and field records for the algal and macroinvertebrate samples destined for laboratory analyses, were loaded into the USGS BioData database, the USGS repository for aquatic bioassessment data (U.S. Geological Survey, 2016).

\section{Aquatic Biota}

Benthic macroinvertebrate and algal communities were sampled by the reach-wide benthos (RWB) method using standard protocols from the Surface Water Ambient Monitoring Program (SWAMP) of the State of the California State Water Resources Control Board (Ode and others, 2016). The RWB method entails sampling throughout the reach at measurement points systematically placed in relation to the 11 primary transects established within the reach. Quantitative subsamples from the 11 transects were then combined into a single composite sample for each of benthic macroinvertebrates and algae. The goal of the RWB method is to represent the ecological condition throughout the reach. The RWB method was used for consistency with other bioassessment data in California and to enable calculation of the California Stream Condition Index (Rehn and others, 2015).
Benthic macroinvertebrate samples were collected before algal samples at a point $1 \mathrm{~m}$ downstream from each primary transect using a D-frame net with $500-\mu \mathrm{m}$ mesh openings (fig. 9). Samples were collected starting with the downstream transect; a 0.09 -square-meter (1-square-foot) sampling station was sited along the transect at a distance 25 percent of wetted width from the left bank (with left and right determined when facing downstream). Moving upstream, the sampling positions within successive transects were alternated between the center, right, and left positions along the transect $(50,75$, and 25 percent of wetted width, respectively), cycling through this order repeatedly. If the targeted section of the stream was too deep to wade, the collection occurred as close to the targeted location as possible. Organisms were separated from the substrate; heavy organisms were removed by hand, larger rocks were scrubbed, and then the streambed was vigorously kicked for 30 seconds while capturing the material in the net.

Material was transferred from the net to a sieve bucket, where transect samples were composited, then emptied onto a double sieve with 0.635 -cm $(0.25$-inch $)$ mesh atop a $500-\mu \mathrm{m}$ mesh. Gravel and detritus were removed from the upper sieve and larger predaceous macroinvertebrates caught on the top sieve were preserved immediately in 95-percent ethanol to reduce the chances of other specimens being consumed or damaged. Large or rare macroinvertebrates, such as crayfish and large mollusks, were photographed and released in accordance with collection permit procedures. The sample remaining on the lower sieve was transferred into one or more 1-L polystyrene wide-mouth bottles filled to no more than 50 percent with the sample material and preserved with 95-percent ethanol. Identification and enumeration of macroinvertebrate taxa (generally to either genus or species taxonomic levels) were completed by the NWQL.

Benthic algal samples were collected at each transect after the benthic macroinvertebrate samples were collected, and at a location approximately $0.25 \mathrm{~m}$ upstream from where the macroinvertebrates were collected. At each sampling location, the dominant substrate type was identified, and a pipe sampler, which was $40 \mathrm{~mm}$ long with an opening of approximately 12 square centimeters, was placed against the substrate. Hard substrates, such as cobble or wood, were lifted from the water, scrubbed with a stifle-bristled brush, and rinsed into the $500-\mathrm{mL}$ wide-mouth polystyrene bottle. In soft substrates, such as sand or detritus, the pipe sampler was pressed into the substrate to a depth of approximately $0.5 \mathrm{~cm}$, and a spatula was slid under the sampler to collect a thin sediment core, which was then rinsed into the sample bottle. For immobile hard substrates, such as boulders, bedrock, or concrete, the tip of a syringe was used to dislodge and suction algae from the area delimited by the pipe sampler, and the material then was emptied into the sample bottle. 


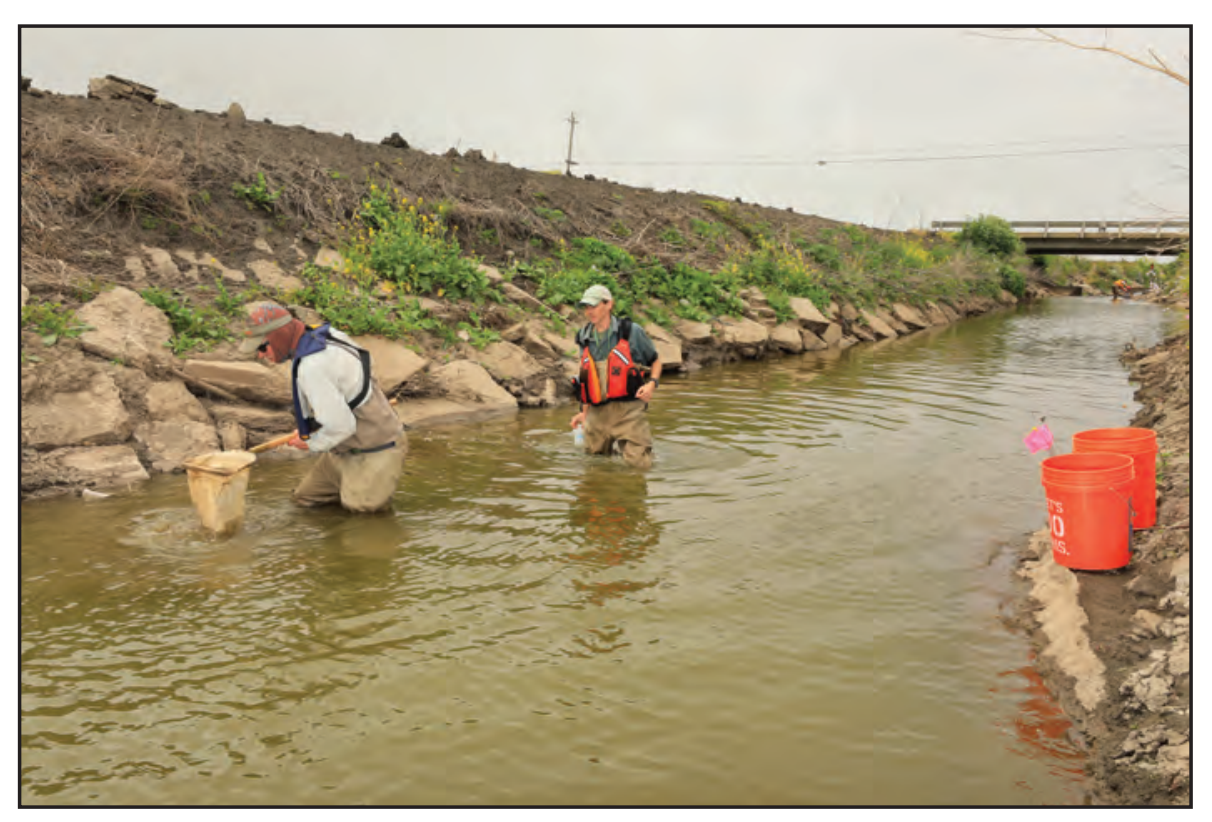

Figure 9. Invertebrate sampling with a D-frame dip net by U.S. Geological Survey personnel. Photograph by Alan Cressler, U.S. Geological Survey.

Five aliquots were removed from the composite sample for various analyses. Four aliquots were filtered onto glass-fiber filters with $0.47-\mu \mathrm{m}$ pore size. Two of these aliquots were placed on dry ice and shipped to the NWQL for analysis of chlorophyll $a$ and pheophytin $a$ (in one sample), and biomass, as ash-free dry mass (in a second sample). The two remaining $0.47-\mu \mathrm{m}$ filtered aliquots were retained frozen as backups in the event of sample loss or damage, or in case additional material was needed for analysis. A fifth aliquot was put into a 2-mL vial and shipped to the Institute of Arctic and Alpine Research (INSTAAR) Diatom Laboratory at the University of Colorado, Boulder, for environmental DNA (eDNA) analysis. The remainder of the composite sample was preserved with buffered formalin at a concentration of approximately 5 percent and sent to the INSTAAR Laboratory for taxonomic identification and enumeration of diatoms.

\section{Physical Habitat}

Following collection of biological samples, the physical habitat of the reach was characterized using USGS protocols (Fitzpatrick and others, 1998). Descriptive and quantitative measurements were made across each primary transect and included geomorphic channel unit type (pool, riffle, or run), stream depth, substrate size at five locations (right and left edges of water, the center of the channel, and midway between channel edges and channel midpoint), stream wetted width, bank height, canopy cover at mid-transect, macrophyte coverage, and the presence of bars, islands, and potential fish habitat features. Substrate size also was measured across the secondary transects, at the same five locations described previously in the text. The surface-water gradient was measured over the entire $150-\mathrm{m}$ reach, and the average slope from the top to the bottom of the reach was determined.

\section{Streambed and Bank Sediment}

During the ecological surveys, one composite sediment sample was collected from the wetted streambed and another composite sample was collected from the stream banks (table 5). At seven sites, wetted streambed samples were collected twice prior to the ecological surveys, during the first and fourth weeks of sampling at these sites (appendix 1, table 1.1). These additional samples were identified as "temporal sediment samples" and, in combination with results from samples collected during the ecological survey, will be used to evaluate how sediment chemistry varied over the sampling period. The sites where temporal sediments were collected are identified with " $\mathrm{T}$ " in the column labeled "Streambed Sediment," under "Ecological Surveys," in table 5. 
The composite streambed-sediment sample was collected by following established USGS protocols (Shelton and Capel, 1994; Radtke, 2005), with some modifications, described as follows. Four-inch (about 10-cm) stainless-steel cylinders and stainless-steel spatulas were used to collect the sediment. Multiple collections of sediment were made from depositional areas along the 150-m sampling reach, targeting locations where fine-grained sediments accumulated (fig. 10). Depositional zones across the reach were sampled in approximate proportion to their bottom surface area. The collection method required pushing the stainless-steel cylinder into the streambed to a depth of $2 \mathrm{~cm}$, then sliding the spatula under the cylinder to support the enclosed streambed core. Each streambed core was lifted gently out of the water to minimize the loss of fine material, and all cores were composited in a large plastic bucket. Approximately 6-10 L of streambed material was collected for the sample. Samples were sieved in the field by using a 2-mm stainless-steel sieve that rested on top of the bucket.

The bulk bed-sediment sample was placed on ice in the field and transported to a USGS research facility in Menlo Park, Calif., where samples were homogenized and split into aliquots for various analyses. Each sample was homogenized by using a kitchen mixer with a stainless-steel bowl and a bread-dough-style paddle operated at low speed. Before the CSQA study, RSQA staff had tested several streambed-sediment samples to determine the mixing time necessary to achieve a reasonably homogeneous sample; about 30 seconds was used for initial mixing, then about 15 seconds for additional mixing between removal of aliquots.
Sample aliquots (2-mm sieve) were shipped chilled to various laboratories for toxicity testing and analyses of organic constituents. Subsamples of streambed sediment were shipped to the USGS Maryland-Delaware-D.C. Water Science Center (WSC) in Baltimore, Md., where they were wet sieved with deionized water using a $63-\mu \mathrm{m}$ polyester sieve. The sieved slurry was collected in glass bowls and dried at $650^{\circ} \mathrm{C}$ for 2 or more days. The dried sediment was removed with a plastic utensil, and aliquots of the fine sediment (sieved at less than $63 \mu \mathrm{m})$ were shipped to multiple laboratories for analysis of inorganic chemicals and carbon. Altogether, sediment samples were collected from 82 sites, and aliquots were analyzed for the following constituents at all sites: current-use pesticides, polycyclic aromatic hydrocarbons (PAH) and other semivolatile compounds, organic wastewater indicators, hormones, halogenated organics (all sieved at $2 \mathrm{~mm}$ ), trace and major elements, organic carbon, radionuclides (all sieved at $63 \mu \mathrm{m}$ ), and grain size. Temporal sediment samples collected at the nine sites (table 5) were analyzed for PAHs, organic wastewater indicators, hormones, radionuclides, trace and major elements, organic carbon, and microcystins.

Bed sediment was used to conduct standardized ambient whole-sediment toxicity tests at the USGS CERC, in accordance with American Society for Testing and Materials International (2016) and U.S. Environmental Protection Agency (2000) methods. Tests were performed with the amphipod Hyalella azteca (28-day exposures) at all sites and with the midge larvae Chironomus dilutus (10-day exposures) at 41 sites (table 5) to measure potential effects of contaminants on survival and growth.

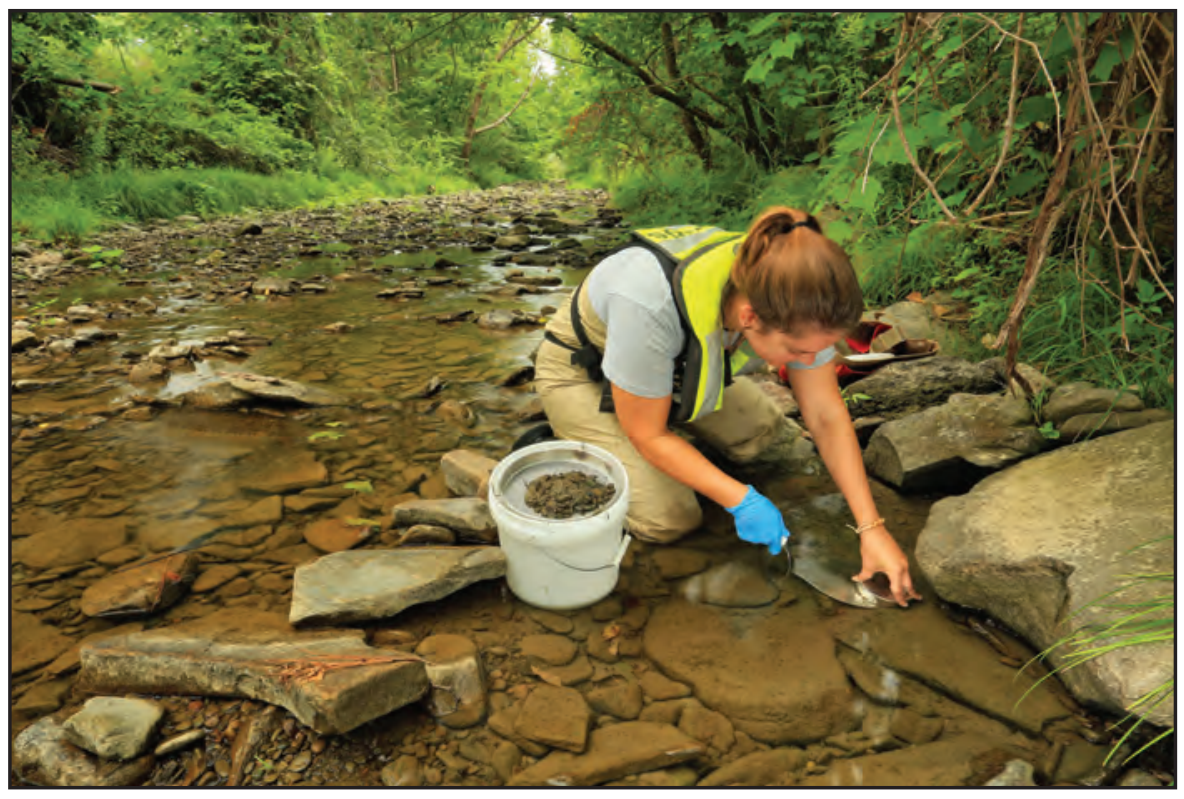

Figure 10. Collection of composite bed-sediment samples from a depositional area along a reach. Photograph by Alan Cressler, U.S. Geological Survey. 
The bank sediment sample was collected at 5-10 locations along the sampling reach where an exposed or eroding bank was observed on either side of the stream. At each location, sediment was collected by using a precleaned plastic trowel to scrape a vertical furrow from above the water line to the top of the exposed bank (about 1-cm depth into the bank). The bank scrapes were composited as a single sample in a 1-L plastic jar. Bank material was stored on wet ice and shipped to the USGS Maryland-Delaware-D.C. WSC in Baltimore, Md., where it was wet-sieved (2-mm) and dried; aliquots were sent to AGAT Laboratories, Mississauga, Ontario, Canada (https:/www.agatlabs.com/index.cfm), for analyses of trace and major elements, and organic carbon.

\section{Sample Analyses}

This section describes chemical analyses of constituents in water and sediment samples, taxonomic analyses of biological samples, and processing of real-time data recorded by data-logging instruments in the field. Most of the chemical analyses of water and sediment samples were conducted by the NWQL or laboratories contracted by the NWQL, using either NWQL or EPA methods and schedules (appendix 2, tables 2.1 to 2.12), which are briefly described in this section; exceptions where other methods were used are noted. Analytical results for constituents in water and sediment from the NWQL were uploaded to the Water-Quality System database (QWDATA) in the National Water Information System (NWIS) of the USGS for storage and archiving. Results for each sample in QWDATA were uniquely identified by station identification number, date, time, and medium code. Additionally, each CSQA sample was labeled with a unique barcode as a backup sample-tracking identifier. Algal and macroinvertebrate samples were analyzed at the NWQL University of Colorado, Boulder, Colo., as described farther onin the text. Continuous data recorded by data-logging instruments, such as streamflow (or stage) and temperature, did not require laboratory analyses so they were processed at the USGS WSC in Sacramento, Calif. (the local WSC).

\section{Comprehensive Stressor Assessments}

\section{Discrete Water-Quality Samples}

Discrete water samples were collected weekly during the WQ index periods (table 5; appendix 1, table 1.1) and analyzed for nutrients, major ions, DOC, ultraviolet absorbance (UVA), and pesticides by the NWQL. Samples for major ions and nutrients were analyzed by the NWQL using various methods (appendix 2, tables 2.1 and 2.2). Specifically, total phosphorus concentrations were determined by colorimetry according to EPA method 365.1 (O'Dell, 1993). Dissolved ammonia, nitrite, and orthophosphate colorimetric analyses are described by Fishman (1993).
Dissolved nitrate-plus-nitrite concentrations were determined by low-level enzyme reduction colorimetry with an automated discrete analyzer, as described by Patton and Kryskalla (2011). Concentrations of dissolved cations were determined by inductively coupled plasma-atomic emission spectroscopy (Fishman, 1993), and concentrations of dissolved anions were determined by ion chromatography, as described by Fishman and Friedman (1989).

For analysis of pesticides, discrete water samples were syringe filtered $(0.7-\mu \mathrm{m}$ pore size glass-fiber) into $20-\mathrm{mL}$ vials. Two pesticide schedules were run on the filtered samples: a broad-spectrum method to determine 225 pesticides and degradates (LCM60, the same pesticide method used in prior RSQA studies; appendix 2, table 2.3.1) and a supplemental schedule of 30 pesticides and degradates (LCM75; appendix 2, table 2.3.2). For both schedules, pesticides were analyzed by direct aqueous injection (DAI) liquid chromatography tandem mass spectrometry (LC-MS/MS; Sandstrom and others, 2016). The original 225 pesticides and pesticide degradates represent a broad range of chemical classes and were selected on the basis of criteria such as the amount of current use, probability of occurrence in streams and groundwater, toxicity to humans or aquatic organisms, and precision of analytical methods (Norman and others, 2012). The supplemental schedule contains additional high-priority pesticides, including neonicotinoid and diamide insecticides and fungicides, and was run on an Agilent model 6495 LC-MS/MS instrument, which is more sensitive than the Agilent 6460 models used for the original method (Sandstrom and others, 2016). For the original LCM60 method, recoveries for most of the 225 analytes ranged from 80 to 120 percent in the water types tested, with relative standard deviations of less than 30 percent. The method detection limits (MDL) ranged from 1 to 106 nanograms per liter (ng/L) for most of the 225 analytes, with current reporting levels (RLDQC) ranging from 2 to $360 \mathrm{ng} / \mathrm{L}$ for these analytes (appendix 2, table 2.3.1). For the supplemental pesticide method LCM75, recoveries were in the 80 - to 120 -percent range for all but one of the 30 pesticide analytes. All 30 analytes had MDLs ranging from 1 to $28 \mathrm{ng} / \mathrm{L}$ and RLDQCs from 2 to $56 \mathrm{ng} / \mathrm{L}$ (appendix 2, table 2.3.2).

Weekly discrete whole-water samples were analyzed for suspended-sediment concentrations at the USGS California Sediment Laboratory in Menlo Park, Calif. Methods for processing suspended-sediment concentrations included use of wet-sieving filtration and are described in Guy (1969) and Knott and others (1993).

Filtered and particulate methylmercury were analyzed in grab water samples (see "Sample Collection and Processing" section) at the USGS Mercury Research Laboratory in Middleton, Wis., by gas chromatographic separation with cold vapor atomic fluorescence spectrometry (DeWild and others, 2002, 2004). Filtered and particulate total mercury were analyzed by oxidation, purge and trap, and cold vapor atomic fluorescence spectrometry (method 1631, revision E; U.S. Environmental Protection Agency, 2002). 
The USGS Mercury Research Laboratory also analyzed DOC in grab samples collected weekly, as well as ultraviolet absorbance at 254 nanometers (UVA254) in grab samples collected concurrently with the mercury samples.

Human-use pharmaceuticals and organic wastewater indicator compounds were analyzed three times for the 6-week sites and once for the 4-week sites (table 5; appendix 1, table 1.1). Pharmaceutical samples were syringe filtered $(0.7-\mu \mathrm{m}$ pore size glass-fiber) into $20-\mathrm{mL}$ vials and analyzed at the NWQL for 113 compounds by DAI LC-MS/MS (appendix 2, table 2.4; Furlong and others, 2008, 2014). Organic wastewater indicator samples were collected as whole water samples into 1-L baked amber glass bottles and analyzed at the NWQL for 69 compounds by gas chromatography mass spectrometry (GC/MS; appendix 2, table 2.5; Zaugg and others, 2006).

Weekly filtered water samples were analyzed for glyphosate by using an enzyme-linked immunosorbent assay (ELISA) at the USGS Texas Water Science Center (Mahler and others, 2017). For all but 11 sites, glyphosate also was analyzed twice during the study period (table 5; appendix 1, table 1.1) at the Kansas OGRL by using solid-phase extraction and LC-MS/MS (Meyer and others, 2009). These data were used to evaluate quality control of the data analyzed by using the ELISA method.

Stable nitrogen $(15 \mathrm{~N})$ and oxygen $(18 \mathrm{O})$ isotopes of nitrate were measured once at all sites (table 5; appendix 1 , table 1.1). Samples were filtered into bottles and frozen until nitrate concentration data were received and then shipped to the USGS Reston Stable Isotope Laboratory in Reston, Virginia. Isotopic analyses were done by following the method of Coplen and others (2012). Dissolved nitrate in water is converted to nitrous oxide $\left(\mathrm{N}_{2} \mathrm{O}\right)$ by denitrifying bacteria, and the nitrous oxide is analyzed for nitrogen and oxygen isotopic abundance by continuous-flow isotope-ratio mass spectrometry.

For analysis of the microcystins class of algal toxins, water samples were processed and analyzed by the OGRL following methods outlined in Loftin and others (2016). Unfiltered samples were lysed by three sequential freeze-thaw cycles at $-20^{\circ} \mathrm{C}$ and $25^{\circ} \mathrm{C}$ and then syringe filtered through $0.7-\mu \mathrm{m}$ glass fiber filters and frozen until analysis. Algal toxins were quantified by using the microcystin ELISA method with a minimum reporting level of 0.10 micrograms per liter.

\section{Polar Organic Compound Integrative Samplers}

The POCIS were processed for analysis of pesticides and pharmaceuticals by using the methods described in
Alvarez (2010) and Van Metre and others (2017a). At CERC, the sample from each POCIS was individually extracted with methanol, and extracts were concentrated to 1 milliliter $(\mathrm{mL})$. Concentrated extracts were sealed in 1-mL amber glass ampules and shipped to the NWQL in Denver, Colo., for analysis. At the NWQL, the extracts were transferred to analytical vials and diluted 1:100, which was required so as not to overwhelm the instrument with the high concentrations of some compounds and to prevent ionization suppression or enhancement of internal standards by the POCIS extracts during LC-MS/MS analysis. Laboratory blank and laboratory-fortified spike samples were prepared by using comparable volumes of methanol and then were processed along with the POCIS extracts. The extracts were analyzed for concentrations of the original 225 pesticides and degradates (LCM60; appendix 2, table 2.3.1), and pharmaceuticals (appendix 2, table 2.4), by using the LC-MS/MS methods described previously for the discrete water samples.

\section{Continuous Streamflow, Stage, and Water Temperature}

Discharge data collected at active USGS streamgages (28 sites) were continuously telemetered to the public in real time via the USGS NWIS database. Four additional sites had streamgages operated by outside agencies, and 19 sites had pressure transducers installed to continuously measure stream stage. At these 19 sites, the raw stage data were processed with the HOBOware graphing and analysis software (Onset Computer Corporation, 2014). This process included applying corrections to the stage values with barometric pressure readings and comparing tape-down measurements from the reference point to the water surface to ensure that the instruments functioned consistently and reliably while they were in service.

The water temperature data were also processed with the HOBOware software (Onset Computer Corporation, 2012); values were checked for outliers that could indicate that the data logger was out of the water, such as with very low flow conditions. The general procedure used to assess the validity of temperature readings that appeared inordinately high was to first review streamflows at the site to determine whether the high temperature values corresponded in time to minimal stage values, then compare these temperature values with those of nearby sites to assess whether the data logger was recording air rather than water temperature. Erroneous water temperature data were subsequently deleted from the data file. 


\section{Focused Studies}

\section{Pesticides from the Small-Volume Pesticide Automated Samplers}

Daily composite samples from the pesticide autosamplers (vials 1 through 7) were analyzed for pesticide concentrations by the EPA OPP Analytical Chemistry Laboratory. Sample splits of the weekly composite sample (vial 9) and the spike sample (vial 8) were analyzed for pesticide concentrations by the NWQL in Denver, Colo., as well as the OPP laboratory. The NWQL analyzed one split sample for 225 current-use pesticides (appendix 2, table 2.3.1) following the methods described earlier in the text (Sandstrom and others, 2016). The OPP laboratory used the same direct aqueous-injection LC-MS/MS method and instrument model (Agilent Model 6460) used by the NWQL (Sandstrom and others, 2016), with similar detection levels, to analyze for 222 pesticides and degradates (appendix 2, table 2.12).

\section{Pesticide Analysis in Biofilms}

Biofilms were analyzed for 94 current-use pesticides (appendix table 2.11) at the USGS Organic Chemistry Research Laboratory (OCRL) in Sacramento, Calif., by using pressurized solvent extraction, cleanup with carbon/alumina (adapted from Hladik and McWayne, 2012), followed by GC-MS/MS analysis (instrument methods from Hladik and others, 2016).

\section{Suspended-Sediment Passive Samplers}

Aliquots of fine fractions (less than $63 \mu \mathrm{m}$ ) of suspended sediment collected from passive samplers and of streambed sediment were analyzed for major and trace elements by AGAT Laboratories, Mississauga, Ontario, Canada, using inductively coupled plasma-optical emission spectroscopy and inductively coupled plasma-mass spectrometry, following dissolution in a mixture of hydrochloric, nitric, perchloric, and hydrofluoric acids (similar to the method documented in Smith and others, 2013). Particle size was measured at the USGS Maryland-Delaware-D.C. WSC in Baltimore, Md., using a laser in situ scattering transmissometer (LISST-100X; Gellis and others, 2018). Additional aliquots of suspended sediment and streambed sediment ( $63-\mu \mathrm{m}$ sieved) were analyzed for radionuclides (lead-210, radium-226, cesium-137, and beryllium-7) at the USGS Sediment Radioisotope Laboratory in Menlo Park, Calif. Radionuclides were analyzed by using a high-resolution gamma spectrometer with an intrinsic germanium detector, following methods described in Van Metre and others (2004).

\section{Ecological Surveys}

\section{Aquatic Biota}

Benthic macroinvertebrate samples were processed by the Biological Unit of the NWQL using a methodology similar to that used in the State of California's Surface Water Ambient Monitoring Program (SWAMP; Woodard and others, 2012). Briefly, the sample was sorted to attain a minimum of 600 organisms, which then were identified to the lowest possible taxonomic level (generally the species or genus level). Quality assurance was verified in both the sorting step and the taxonomic step by a second taxonomist, following the QA/QC procedures in Moulton and others (2000). Taxonomic and enumeration results were uploaded to BioData (U.S. Geological Survey, 2016).

Benthic algal samples were processed in the field by filtering onto $0.47-\mu \mathrm{m}$ glass-fiber filters. The filters were analyzed at the NWQL for chlorophyll $a$ and pheophytin $a$ by using EPA method 445.0 and for algal ash-free dry mass by using USGS method B-3520-85 (appendix 2, table 2.10; Britton and Greeson, 1989; Arar and Collins, 1997).

Algal samples preserved with formalin were analyzed for diatom community composition and abundance at the INSTAAR laboratory at the University of Colorado, Boulder, following NAWQA protocols (Charles and others, 2002) with the following modification. Four replicate slides of the diatoms were made by using Battarbee chambers to obtain random distribution of cells on cover slips (Battarbee, 1973). A pre-count collection of voucher flora was created, based on examination of 80 percent of the algal slides. The voucher flora included images of all taxa encountered, with a greater number of images for rare and previously unknown taxa. The images were sorted into operational taxonomic units (OTUs) and assigned OTU codes. Samples and their order of analysis were randomly assigned to two analysts. Ten percent of samples were reanalyzed by each analyst, and 10 percent of samples were analyzed in cross comparison. Finally, OTU codes were translated into formal scientific names following the taxonomy in the USGS BioData program and Diatoms of the United States (Spaulding and others, 2010). Voucher slides, digested material, and the voucher flora were archived at INSTAAR. The soft algae fractions of the samples were stored at INSTAAR for possible analysis at a later time.

\section{Physical Habitat}

Data collected from habitat surveys were recorded on electronic field forms and reviewed later in the office by USGS staff. Any values on the field forms that were suspect (such as typographical errors) were resolved, and the data were archived for a future data release. 


\section{Streambed and Bank Sediment}

Aliquots of bulk (homogenized, 2-mm sieved) streambed sediment were analyzed for grain size at the USGS Missouri Sediment Laboratory in Rolla, Mo. Major and trace elements and organic carbon were analyzed in aliquots of streambed sediment (less than $63 \mu \mathrm{m}$ ) by AGAT Laboratories using the same methods described earlier in the text for the suspended-sediment passive samples. Aliquots of the streambed samples (less than $63 \mu \mathrm{m}$ ) were analyzed for radionuclides at the USGS Sediment Radioisotope Laboratory in Menlo Park, Calif., using the same methods described earlier in the text for the suspended-sediment passive samples.

Aliquots of streambed sediment (less than $2 \mathrm{~mm}$ ) were sent to the USGS NWQL for analysis of three organic contaminant schedules. One aliquot was analyzed for organic wastewater indicator compounds by using accelerated solvent extraction (ASE), solid-phase extraction cleanup, and GC/MS (appendix 2, table 2.6; Burkhardt and others, 2006). A custom method was used for selected organohalogens in sediment (chlorinated and brominated compounds including insecticides, polychlorinated biphenyls [PCBs], and polybrominated diphenyl ethers [PBDEs]), in which the sample was extracted by ASE, followed by solid-phase extraction cleanup and analysis by electron-capture negative ionization mode $\mathrm{GC} / \mathrm{MS}$ with selected ion monitoring (appendix 2, table 2.8; reported in Mahler and others, 2009; Wagner and others, 2014). Hormone compounds in bed sediment were analyzed using a custom method (appendix 2, table 2.9) consisting of pressurized solvent extraction, then two solid-phase extraction steps to isolate the compounds and further clean the extract (Fischer and others, 2015), followed by compound derivatization and analysis by gas chromatography with tandem mass spectrometry (GC-MS/MS) using procedures similar to Foreman and others (2012). An additional aliquot of streambed sediment (less than $2 \mathrm{~mm}$ ) was sent to RTI Laboratories, Livonia, Michigan (https://rtilab.com/), for analysis of 17 PAHs using EPA method 8270D (U.S. Environmental Protection Agency, 2014), with extraction by ASE and analysis by GC/MS with selected ion monitoring (appendix 2, table 2.7). Another aliquot of streambed sediment (less than $2 \mathrm{~mm}$ ) was analyzed for 118 pesticides (most of which are in current use) at the USGS OCRL in Sacramento, Calif., by using pressurized solvent extraction, followed by a series of cleanup procedures (gel permeation chromatography and SPE with either carbon/ alumina or Florisil), followed by GC/MS analysis (Hladik and McWayne, 2012).

Bank material (less than $2 \mathrm{~mm}$ ) was analyzed for grain size at the USGS Maryland-Delaware-D.C. WSC, Baltimore, Md. Major and trace elements and organic carbon were analyzed by AGAT Laboratories, Mississauga, Ontario, Canada.

\section{Sediment Toxicity Testing}

Whole sediment toxicity tests were conducted with the amphipod Hyalella azteca (28-day exposures) and with the midge Chironomus dilutus (10-day exposures), following methods in U.S. Environmental Protection Agency (2000) and American Society for Testing and Materials International (2016), with detailed test conditions noted in Moran and others (2017). As much as $1.8 \mathrm{~L}$ (assuming a 50/50 split of solids and liquids) of the composited streambed sediment was used for toxicity testing. Testing for each species included endpoints of survival, weight, and biomass of test organisms. Exposures were conducted at $23{ }^{\circ} \mathrm{C}$ in $300-\mathrm{mL}$ beakers containing $100 \mathrm{~mL}$ of sediment with two volume additions per day of overlying water. Ten organisms were exposed in each beaker with four replicate beakers per site. Endpoints were survival, growth (dry weight for amphipods and ash-free dry weight for midge), and biomass for each test species. Response of each endpoint for each species was compared to a standard control sediment from Spring River, Mo. (Besser and others, 2015). Comparisons also were made using a reference envelope approach, which addresses the variability observed from reference sediments that were primarily from undeveloped watersheds, had minimal levels of contamination, and showed acceptable biological performance, as defined in Kemble and others (2013). 


\section{Quality Assurance and Quality Control}

This section describes the QA/QC procedures for stressor assessments (specifically, constituents in discrete water samples, POCIS samples, and streambed-sediment samples), as well as for overall project-specific training and management. QA/QC procedures for the focused studies were described in the "Sample Collection and Processing" section. For the ecological surveys, QA/QC procedures are built into the taxonomic identification process and are described in the "Sample Analyses" section.

Quality assurance/quality control for project data is an iterative process that begins when samples are collected, continues through the establishment of sample records in NWIS, and ends with the final acceptance of data as reviewed and approved or, in rare cases, rejected. This process allows for a continuous review of records by field personnel, laboratory analysts, RSQA team data managers, QW specialists, and other team members. Specific database scripts were developed to check sample coding logic and to generate data tables in multiple formats for data review and confirmation.

Quality assurance/quality control procedures maintain the integrity, accuracy, and legal defensibility of results from data collection and assessment. Documented USGS QA/ QC policies and procedures for environmental sampling were implemented in the study to ensure that the data can be interpreted properly and are scientifically defensible (Mueller and others, 1997; U.S. Geological Survey, 2006). Quality control samples were collected to identify, quantify, and document bias and variability in data that result from the sampling procedure (through field QC sampling) and laboratory procedures (through laboratory QC sampling). Field QC sampling is used to estimate bias and variability from sample collection, processing, shipping, and handling of samples. Laboratory QC sampling documents the variability of analytical methods and sample preparation in the laboratory.

To ensure that all field crews followed consistent sample collection and processing procedures, classroom training was held for field personnel before the sampling period. In addition, all personnel worked through a full suite of sample collection and sample processing procedures in the field just before the start of the CSQA sampling period. To maximize efficiency in the field, all sampling scheduling and preparation of weekly sampling supplies were handled centrally by USGS personnel who had provided similar support to previous RSQA studies. Sample bottle packs were assembled at the USGS Sacramento Field Office by USGS staff at least 1 week before sampling. The bottle packs consisted of the necessary bottles, filters, preservatives, labels, and ASRs for each stream site. Centralizing the assembly and distribution of sample bottle packs helped ensure that correct sample coding, sample schedules, and timing of QC samples matched the sampling plan and reduced errors in the sample login process at the analytical laboratories.
The QC samples for constituents measured in water included field blanks, field matrix spikes, and replicates (table 6; appendix 1, table 1.1). The QA/QC plan was designed to have 5 percent or more QC samples for inorganics and 10 percent for organics, to ensure that QC samples were distributed across the region (spatially) and study period (temporally), and to assign collection of QC samples to every field crew. Field blanks were used to test whether field cleaning procedures would adequately remove any equipment contamination introduced by sampling at previous sites and to ensure that sample collection, processing, handling, and shipping did not result in contamination (Mueller and others, 1997; U.S. Geological Survey, 2006). Field replicates provided a measure of the variability introduced during sample processing and analysis (Mueller and others, 1997; U.S. Geological Survey, 2006). When water samples were collected from the churn, two aliquots of water were split from the churn into separate containers and analyzed as field replicates (split replicates). When grab samples were collected, replicates were collected sequentially directly from the stream. Field and laboratory matrix spikes were used to assess the potential bias for analytes in a particular sample matrix. Bias is estimated from spiked samples by calculating the percentage of the added analyte (spike material) that is measured (recovered) in the sample at the laboratory (Mueller and others, 1997; U.S. Geological Survey, 2006). Recovery can be either greater than or less than 100 percent, so the bias can be either positive or negative. However, matrix interference and analyte degradation generally result in a negative bias.

Field blanks were collected once from 16 to 18 sites for each of the basic laboratory schedules (major ions, nutrients, dissolved organic carbon, pesticides, and glyphosate by immunoassay; table 6) that were part of the comprehensive stressor assessments. The targeted number of total QC samples per analytical schedule was 10 percent for organic contaminants and 5 percent for inorganic constituents, with QC samples distributed throughout the study area, the study period (appendix table 1.1), and among the nine field crews. CSQA field QC samples represented 5-8 percent of environmental samples for major ions, nutrients, and DOC; 11 percent for mercury; and 16-18 percent for the broad-spectrum pesticide methods, glyphosate by LC-MS/MS, pharmaceuticals, and organic wastewater indicators, all of which met the frequency recommendations (table 6).

For pesticides, pharmaceuticals, and organic wastewater indicators, field blanks and field replicates each represented about 3-5 percent of the environmental samples (table 6). Field matrix spikes were created for analyses of all organic contaminants, with the exception of glyphosate analysis by immunoassay. The frequency of the field matrix spikes ranged from 5 to 10 percent for organic contaminants, depending on the laboratory schedule (table 6). For glyphosate by immunoassay, laboratory matrix spikes (not shown in table 6) were analyzed, which represented an additional 2 percent of environmental samples. 
Table 6. Summary counts of environmental, field blank, replicate, and spike samples of stream water from the 85 stream sites sampled in the California Stream Quality Assessment study of the U.S. Geological Survey National Water-Quality Assessment Project in 2017.

[OGRL, U.S. Geological Survey Organic Geochemistry Research Laboratory in Lawrence, Kansas; QC, quality control; —, not applicable]

\begin{tabular}{|c|c|c|c|}
\hline \multirow[t]{2}{*}{$\begin{array}{l}\text { Type of } \\
\text { sample }\end{array}$} & \multirow[t]{2}{*}{$\begin{array}{c}\text { Sample } \\
\text { counts }\end{array}$} & \multicolumn{2}{|c|}{$\begin{array}{c}\text { Ratio of } \mathbf{Q C} \text { to } \\
\text { environmental samples } \\
\text { (percent) }\end{array}$} \\
\hline & & Actual & Recommended 1 \\
\hline \multicolumn{4}{|c|}{ Laboratory schedule: Major ions } \\
\hline Environmental & 488 & 7.6 & 5 \\
\hline Blank & 16 & 3.3 & - \\
\hline Replicate & 21 & 4.3 & - \\
\hline Spike & 0 & 0 & - \\
\hline \multicolumn{4}{|c|}{ Laboratory schedule: Nutrients } \\
\hline Environmental & 488 & 7.6 & 5 \\
\hline Blank & 16 & 3.3 & - \\
\hline Replicate & 21 & 4.3 & - \\
\hline Spike & 0 & 0 & - \\
\hline \multicolumn{4}{|c|}{ Laboratory schedule: Dissolved organic carbon } \\
\hline Environmental & 485 & 5.0 & 5 \\
\hline Blank & 17 & 3.5 & - \\
\hline Replicate & 20 & 4.1 & - \\
\hline Spike & 0 & 0 & - \\
\hline \multicolumn{4}{|c|}{ Laboratory schedule: Pesticides } \\
\hline Environmental & 488 & 17.4 & 10 \\
\hline Blank & 18 & 3.7 & - \\
\hline Replicate & 22 & 4.5 & - \\
\hline Spike & 45 & 9.2 & - \\
\hline \multicolumn{4}{|c|}{ Laboratory schedule: Supplemental pesticides } \\
\hline Environmental & 488 & 17.6 & 10 \\
\hline Blank & 18 & 3.7 & - \\
\hline Replicate & 21 & 4.3 & - \\
\hline Spike & 47 & 9.6 & - \\
\hline \multicolumn{4}{|c|}{ Laboratory schedule: Glyphosate (immunoassay) } \\
\hline Environmental & 487 & 7.6 & 10 \\
\hline Blank & 16 & 3.3 & - \\
\hline Replicate & 21 & 4.3 & - \\
\hline Spike & 20 & 20 & - \\
\hline
\end{tabular}

\begin{tabular}{|c|c|c|c|}
\hline \multirow[t]{2}{*}{$\begin{array}{l}\text { Type of } \\
\text { sample }\end{array}$} & \multirow[t]{2}{*}{$\begin{array}{l}\text { Sample } \\
\text { counts }\end{array}$} & \multicolumn{2}{|c|}{$\begin{array}{c}\text { Ratio of } \mathrm{QC} \text { to } \\
\text { environmental samples } \\
\text { (percent) }\end{array}$} \\
\hline & & Actual & Recommended \\
\hline \multicolumn{4}{|c|}{ Laboratory schedule: Pharmaceuticals } \\
\hline Environmental & 233 & 16.3 & 10 \\
\hline Blank & 11 & 4.7 & - \\
\hline Replicate & 13 & 5.6 & - \\
\hline Spike & 14 & 6.0 & - \\
\hline
\end{tabular}

\begin{tabular}{|c|c|c|c|}
\hline \multicolumn{4}{|c|}{ Laboratory schedule: Organic wastewater indicators } \\
\hline Environmental & 233 & 15.9 & 10 \\
\hline Blank & 12 & 5.2 & - \\
\hline Replicate & 12 & 5.2 & - \\
\hline Spike & 13 & 5.6 & - \\
\hline \multicolumn{4}{|c|}{ Laboratory schedule: Glyphosate (OGRL) } \\
\hline Environmental & 147 & 15.0 & 10 \\
\hline Blank & 9 & 6.1 & - \\
\hline Replicate & 13 & 8.8 & - \\
\hline
\end{tabular}

\begin{tabular}{lrcc}
\hline \multicolumn{4}{c}{ Laboratory schedule: Mercury } \\
\hline Environmental & 244 & 11.1 & 5 \\
Blank & 14 & 5.7 & - \\
Replicate & 13 & 5.3 & - \\
Spike & 0 & 0 & - \\
\hline \multicolumn{4}{l}{ Laboratory schedule: Algal toxins } \\
\hline Blankironmental & 84 & 12 & 10 \\
Replicate & 5 & 6 & - \\
Spike & 5 & 6 & - \\
\hline
\end{tabular}

\begin{tabular}{lccc}
\multicolumn{4}{c}{ Laboratory schedule: Isotopes } \\
\hline Environmental & 84 & 10.7 & 5 \\
Blank & 0 & 0 & - \\
Replicate & 9 & 10.7 & - \\
Spike & 0 & 0 & - \\
\hline
\end{tabular}

${ }^{1}$ Recommendations for total QC samples are 10 percent of environmental samples for organic constituents and 5 percent for inorganic constituents.

${ }^{2}$ Although no field matrix spike samples were analyzed, laboratory matrix spike samples (not shown in this table) represented an additional 4 percent of environmental samples. 
Quality assurance/quality control procedures generally were simpler for sediment samples than for water samples and included the use of field replicates (splits of composite bed-sediment samples) as well as (for pesticides) laboratory blanks and laboratory matrix spikes. In addition, two additional streambed samples (for a total of three samples per site) were collected at a subset of seven sites to assess temporal variability in bed-sediment contaminants. Counts of environmental and QC samples for constituents in bed sediment are shown in table 7. Field replicates represented 11 percent of environmental samples for pesticides, PAHs, organic wastewater indicators, hormones, halogenated organic compounds, trace elements, radionuclides, and grain size. For pesticides in sediment, the laboratory blanks and laboratory matrix spikes analyzed each represented about 7 percent of environmental samples (table 7).

Quality assurance included maintaining standardized sample collection and handling protocols among all field personnel as described in the National Field Manual (U.S. Geological Survey, variously dated) for water and sediment sampling and in Moulton and others (2002) for ecological sampling. All sampling and handling protocols were reviewed by field personnel involved in the CSQA study during training courses, before field work began. Additionally, several programs exist in the USGS Quality Systems Branch to help document the quality of project results. For laboratory analyses conducted by the NWQL, documented QC included double-blind analyses of blanks for organic and inorganic constituents in water and provision of graphical and tabular control data for the analytical lines. Field personnel involved in the CSQA study are tested annually to verify their proficiency in collecting field data, including temperature, $\mathrm{pH}$, dissolved oxygen, alkalinity, and specific conductance.

For POCIS samples, field QC samples included replicates and blanks, and their total number represented 20 percent of the environmental POCIS samples (appendix 1, table 1.5). Field replicate samples were obtained by deploying a second canister containing 2 POCIS, one for each of the pesticide and pharmaceutical schedules, at seven sites (11 percent). At six sites ( 9 percent), the environmental POCIS sample was accompanied by a field blank that was used to assess any accumulation of target and nontarget compounds from the air during shipment and deployment. All POCIS and blank canisters were stored on ice during transport to and from the field location. The blank canisters were open to the air at the same time and place as the environmental POCIS were exposed to air during deployment and retrieval. Between deployment and retrieval of the environmental POCIS samples, the POCIS blank canisters were kept sealed and stored between -20 and $0{ }^{\circ} \mathrm{C}$.

Water-quality data from each sampling event were reviewed for completeness, precision, bias, and transcription errors when received from the laboratory as part of the QA/QC procedures. Water-quality and sediment-quality data were stored in the NWIS database; the data are available for retrieval at https://nwis.waterdata.usgs.gov/nwis/sw and through the data retrieval application at the RSQA project website (https://webapps.usgs.gov/RSQA/). The NWQL provides all QA/QC documentation for analytical services at https://nwql.usgs.gov/Public/quality.shtml. Data also will be published in ScienceBase.

Temperature calibration checks were completed on the HOBO U20 and U22 units before deployment and after retrieval according to the USGS National Field Manual (Wilde, 2008). The USGS WSC in Columbus, Ohio, maintains a commercially refrigerated water bath specifically for the purposes of thermometer and temperature sensor calibration. The bath is calibrated to a National Institute of Standards and Technology (NIST)-certified thermometer, and calibration checks on temperature sensors are conducted at 3.00, 10.05, $15.03,25.05,30.04$, and $40.03{ }^{\circ} \mathrm{C}$. Units found to be within their performance range are certified for deployment. Pressure transducer units also were checked that their barometric pressure reading was within 1 percent of a NIST-certified barometer before deployment and after retrieval. This was done at the atmospheric pressure for the USGS WSC in Columbus, Ohio. Air pressure transducer readings from sensors were not checked against NIST-certified barometers in the field. However, continuous readings were verified during the correction process against water-quality sonde readings measured during discrete sampling.

Continuous temperature and pressure data processing was done by a single person to maintain consistency within the CSQA study and included the following steps. Data were evaluated for erroneous readings at the start of deployment and the end of retrieval for situations where the device was actively logging data while in route to the site or stream. These data were removed. The data were then plotted to identify significant spikes or dips in the record, including possible loss of record. Spikes in the data that were inconsistent with 
Table 7. Summary counts of environmental, field replicate, field spike, and temporal samples of bed sediment collected from 82 stream sites in the California Stream Quality Assessment study of the U.S. Geological Survey National Water-Quality Assessment Project in 2017.

[Temporal bed sediment samples, which were collected at 7 sites (table 5), are not included in environmental sample counts and percentages of QC samples. Abbreviations: NAV, not available; QC, quality control; - , not applicable]

\begin{tabular}{|c|c|c|c|}
\hline \multirow[t]{2}{*}{ Type of sample } & \multirow[t]{2}{*}{ Sample counts } & \multicolumn{2}{|c|}{$\begin{array}{c}\text { Ratio of QC to environmental } \\
\text { samples } \\
\text { (percent) }\end{array}$} \\
\hline & & Actual & Recommended 1 \\
\hline \multicolumn{4}{|c|}{ Laboratory schedule: Pesticides } \\
\hline Environmental & 82 & 26 & 10 \\
\hline Replicate & 9 & 11 & - \\
\hline Blank $^{2}$ & 6 & 7.3 & - \\
\hline Spike $^{2}$ & 6 & 7.3 & - \\
\hline Temporal & NAV & NAV & - \\
\hline \multicolumn{4}{|c|}{ Laboratory schedule: Polycyclic aromatic hydrocarbons } \\
\hline Environmental & 82 & 11 & 10 \\
\hline Replicate & 9 & 11 & - \\
\hline Temporal & 14 & 0 & - \\
\hline \multicolumn{4}{|c|}{ Laboratory schedule: Organic wastewater indicators } \\
\hline Environmental & 82 & 11 & 10 \\
\hline Replicate & 9 & 11 & - \\
\hline Temporal & 12 & 0 & - \\
\hline \multicolumn{4}{|c|}{ Laboratory schedule: Hormones } \\
\hline Environmental & 82 & 11 & 10 \\
\hline Replicate & 9 & 11 & - \\
\hline Temporal & 13 & 0 & - \\
\hline \multicolumn{4}{|c|}{ Laboratory schedule: Halogenated organics } \\
\hline Environmental & 82 & 11 & 10 \\
\hline Replicate & 9 & 11 & - \\
\hline Temporal & 12 & 0 & - \\
\hline \multicolumn{4}{|c|}{ Laboratory schedule: Trace elements } \\
\hline Environmental & 81 & 11 & 5 \\
\hline Replicate & 9 & 11 & - \\
\hline Temporal & 14 & 0 & - \\
\hline \multicolumn{4}{|c|}{ Laboratory schedule: Grain size } \\
\hline Environmental & 82 & 11 & 5 \\
\hline Replicate & 9 & 11 & - \\
\hline Temporal & 0 & 0 & - \\
\hline \multicolumn{4}{|c|}{ Laboratory schedule: Radionuclides } \\
\hline Environmental & 82 & 11 & 5 \\
\hline Replicate & 9 & 11 & - \\
\hline Temporal & 14 & 0 & - \\
\hline
\end{tabular}

${ }^{1}$ Recommendations for total QC samples are 10 percent of environmental samples for organic constituents and 5 percent for inorganic constituents.

${ }^{2}$ Laboratory blanks and laboratory matrix spikes were analyzed for pesticides in bed sediment. observed changes in air temperature, weather events, or flow, or that were not corroborated with other nearby records, were removed from the record. 


\section{Data-Management Procedures}

An important goal of data management for the CSQA study is to have the data reviewed, approved, and stored in a USGS approved database that is appropriate for the specific type of data (for example, water quality, streamflow or stage, and biological). Because CSQA sampling sites were only in California, data entry and retrieval for sites were managed by the local WSC. The NWIS station number that is used to identify a site (table 1) is the master indexing and retrieval element for accessing data specific to the site. The NWIS database is the repository for most of the water-quality and streamflow data, which constitute most of the CSQA data. Additionally, a data-management team composed of national RSQA staff and local CSQA staff was created to facilitate the data-management process. Centralization of the data-management process was adopted to ensure consistency among all RSQA study areas and the respective WSCs. Nine main steps were implemented in the data-management process:

1. Sampling matrix and sample coding design;

2. Electronic field form use, including barcoding;

3. Sample status checks at all laboratories;

4. NWIS sample record checks;

5. Data transfer from laboratory to NWIS;

6. Establishment of project networks;

7. Sample coding and field parameter checks;

8. Data quality checks;

9. Approval of data in NWIS and other databases, as appropriate.

Sites for the CSQA study were assigned the appropriate network designations in NWIS ProjectNetworks (Dupré and others, 2013), which allows integration with similar sites across many regions and designation of the network type in NWIS. These network designations were obtained from the project planning documents and, where possible, kept consistent with other network designations used in previous regional studies. ProjectNetworks documentation enabled local CSQA personnel to establish their sites in NWIS ProjectNetworks.

Before the start of sampling, the manager of the data-management team (henceforth, data manager) prepared a matrix that would be the sampling design and coding plan for all aspects of the field activities. The sampling matrix distributed QC samples approximately equally across sites, sample teams, and time periods for optimum coverage. The matrix also served as a summary for the type, frequency, and location of environmental and QC samples to be collected (appendix 1, table 1.1). A sample coding scheme was developed by the data manager that was used by the CSQA sampling teams to ensure a well-structured and manageable dataset. Additionally, training and written guidelines for sampling coding were made available to sampling teams before the start of sampling.

Most of the CSQA sampling teams used the Personal Computer Field Form (PCFF) version 7.2 software created by the USGS, which provides electronic field forms for data collection at sampling sites. Some field teams did not use the PCFF; in these cases, field data were recorded on paper field forms and then transferred to electronic digital forms in the office. In any case, the use of PCFF did not preclude the use of paper field forms when sampling; a two-page standardized form for CSQA water-quality field notes was routinely used at all sites to record basic site conditions when samples were collected and to affix bar codes that identified the samples. The bar codes are unique identifiers used to associate specific sample types with a site and the sampling event. The PCFF software streamlines the process of uploading (logging in) field data and sampling codes to NWIS by automatically generating the batch load files required by NWIS (qwsample and qwresult) and thereby improves the efficiency of data flow from field and laboratory to database. The information uploaded to NWIS for each sample is stored under a unique number associated with that sample, as are all results received later from the laboratory. In addition, the automation of data upload to NWIS limits the incidence of transcription errors that can occur during the manual entry of data into NWIS. The field-supply manager provided ASRs to the sampling teams each week along with the corresponding bottle sets.

Sample shipment schedules were established before the start of sampling for CSQA, and generally shipments were made twice per week (appendix 1, table 1.4). Sampling teams and other local WSC personnel were responsible for the shipment process. The data manager tracked the shipments to verify that the shipped samples were received at each laboratory (1) within the correct holding times, (2) in the proper condition (for example, chilled samples received at the appropriate temperature of $4{ }^{\circ} \mathrm{C}$ or less), and (3) with proper documentation. The data manager worked with the laboratories to correct problems with mislabeled samples or ASRs in a timely manner and to communicate problem-resolution approaches to local WSC personnel. During this process, the data manager also established the 
connection between the USGS Laboratory Information Management System used to transfer sample results and the NWIS database used to store and receive sample results.

During sampling and the corresponding establishment of sample records in NWIS, the data manager inspected sample coding and procedures to ensure that sample records were established properly and in a consistent manner. Sample coding or the procedures of coding were modified if found to be inaccurate or inconsistent. These modifications involved changes or corrections to sample time offsets, sample type coding, or other documentation at the NWQL or in NWIS. Modifications in sample coding or procedures related to data management or sample submittal were communicated immediately to sampling teams to ensure that appropriate adjustments were made before the next sampling.

Most of the laboratories used for CSQA sample analysis transmitted sample results through the Water Quality Data Exchange (QWDX) for automatic upload into the NWIS database. For those laboratories without the ability to use QWDX, sample results were loaded into NWIS by using manually created batch files. Batch files were created by the data manager upon receipt of electronic data from the laboratory and were loaded into NWIS by the data manager or the database administrator for the local WSC. The data manager verified that the batch files of data were properly loaded into NWIS.

Similarly, the Ecology Sampling Coordinator verified that algal, macroinvertebrate, and habitat data were properly loaded into BioData and into the ScienceBase digital data repository supported by the USGS (https://www.sciencebase.gov/catalog/ item/5e15f326e4b0ecf25c55c034). Data files provided through email by laboratories and data not applicable to NWIS (for example, CERC toxicity data) were stored electronically in the RSQA team database rather than in NWIS. These data, and data such as QC sample results not publicly available through NWIS, will be released separately through USGS ScienceBase.

After sampling was completed, the data manager inspected the NWIS sample records for completeness regarding field data collection, including stream measurements (streamflow, stage, sampling points, stream width, and so forth), field parameters ( $\mathrm{pH}$, air and water temperature, specific conductance, dissolved oxygen), and sample coding (sample purpose, purpose of site visit, sampling method, sampler type, and multiple QC-related sample codes). Manual checks were made for each sample, and any needed corrections were communicated to WSC personnel.

National RSQA staff scientists reviewed the water-quality and sediment-quality results received from the laboratories. The water-quality data reviews included identification and review of extremes in the data (outliers), inconsistencies or unexpected results, major differences between environmental samples and replicates, detected values in blanks, surrogate recoveries, and analyte recoveries in spike samples, as well as review of sample coding. The RSQA staff scientists communicated requests for reruns, reloads, and verification of results to the laboratory; they worked closely with the data manager to verify completeness and accuracy of sample results. After completion of data review by RSQA staff, the database administrator at the USGS California WSC in Sacramento, Calif., changed the data quality indicator (DQI), depending on the results of the review, for groups of parameter types to "reviewed and accepted" (R) or, in a few rare cases, "reviewed and rejected" (Q).

Water-quality, sediment, and ecological survey data will be available to the public in NWIS and ScienceBase, as well as at the RSQA mapping and data application website, which allows mapping, querying, and data downloads (https://webapps.usgs.gov/RSQA/\#!/download). 
Continuous stage and temperature data measured by the HOBO U20-001-04 digital loggers were reviewed and corrected as described in the "Quality Assurance and Quality Control" section and will be published in ScienceBase.

\section{Summary}

This report describes the design and methods used during an intensive regional study, designated by the California Stream Quality Assessment (CSQA), to assess stream quality in the central coastal region of California, United States, where the larger valleys contain intense urbanization or agriculture, or both. This is the last of five regional stream-quality assessment (RSQA) studies conducted by the U.S. Geological Survey (USGS). The overall goal of the RSQA studies is to improve the understanding of multiple water-quality stressors that affect wadeable streams throughout the region by evaluating relations between these stressors and biological indicators of stream health. The RSQA study design has three elements: comprehensive stressor assessments, focused studies, and ecological surveys. In CSQA, 85 wadeable stream sites were selected throughout the Central California Foothills and Coastal Mountains (an EPA Level III ecoregion) to determine the occurrence and levels of multiple stressors, and to assess stream habitat and the condition of aquatic algal and macroinvertebrate communities during spring 2017. A wide variety of potential stressors was assessed in the CSQA study, including nutrients, trace elements and major ions in water, several classes of organic contaminants in water and sediment, suspended sediment, and flow alteration. Data on water and sediment-quality constituents came from discrete, continuous, and integrative samples collected at all sites, supplemented by in-depth characterizations of selected stressors from focused studies conducted at subsets of sites.

The 85 sites were divided into a Southern group (36 sites) and a Northern group (49 sites). Water quality was measured at all sites during either a 4- or 6-week period from mid-March to mid-May 2017, with sampling at the Southern sites starting 3 weeks earlier than at the Northern sites. After the water-quality sampling period, an ecological survey was conducted at each site in May 2017 to assess algal and macroinvertebrate communities, the presence of harmful algal toxins, contaminants in sediment, sediment toxicity to amphipods and midge larvae, and the physical habitat of the stream. This report describes procedures used for sample collection and processing, sample analyses, quality assurance and quality control, and data management.

\section{References Cited}

Alvarez, D.A., 2010, Guidelines for the use of the semipermeable membrane device (SPMD) and the polar organic chemical integrative sampler (POCIS) in environmental monitoring studies: U.S. Geological Survey Techniques and Methods 1-D4, 28 p., https://doi.org/10.3133/tm1D4.

American Society for Testing and Materials International, 2016, Standard test method for measuring the toxicity of sediment-associated contaminants with freshwater invertebrates, in Annual Book of American Society for Testing and Materials International Standards Volume 11.06: West Conshohocken, Pa., American Society for Testing and Materials International, ASTM E1706-16, https://www.astm.org/Standards/E1706.htm.

Arar, E.J., and Collins, G.B., 1997, Method 445.0-In vitro determination of chlorophyll $a$ and pheophytin $a$ in marine and freshwater algae by fluorescence (rev. 1.2, September 1997): Cincinnati, Ohio, U.S. Environmental Protection Agency, National Exposure Research Laboratory, Office of Research and Development, 22 p., https://cfpub.epa.gov/si/si_public_record_report.cfm?Lab= NERL\&dirEntryId=309417.

Battarbee, R.W., 1973, A new method for the estimation of absolute microfossil numbers, with reference especially to diatoms: Limnology and Oceanography, v. 18, no. 4, p. 647-653, https://doi.org/10.4319/lo.1973.18.4.0647.

Besser, J.M., Ingersoll, C.G., Brumbaugh, W.G., Kemble, N.E., May, T.W., Wang, N., MacDonald, D.D., and Roberts, A.D., 2015, Toxicity of sediments from lead-zinc mining areas to juvenile freshwater mussels (Lampsilis siliquoidea) compared to standard test organisms: Environmental Toxicology and Chemistry, v. 34, no. 3, p. 626-639, https://doi.org/10.1002/etc.2849.

Britton, L.J., and Greeson, P.E., eds., 1989, Gravimetric method for biomass, in Methods for collection and analysis of aquatic biological and microbiological samples: U.S. Geological Survey Techniques of Water-Resources Investigations, book 5, chap. A4, p. 139-140, https://doi.org/10.3133/twri05A4.

Brown, L.R., May, J.T., Rehn, A.C., Ode, P.R., Waite, I.R., and Kennen, J.G., 2012, Predicting biological condition in southern California streams: Landscape and Urban Planning, v. 108, no. 1, p. 17-27, https://doi.org/10.1016/j.landurbplan.2012.07.009. 
Burkhardt, M.R., Zaugg, S.D., Smith, S.G., and ReVello, R.C., 2006, Determination of wastewater compounds in sediment and soil by pressurized solvent extraction, solid-phase extraction, and capillary-column gas chromatography/mass spectrometry: U.S. Geological Survey Techniques and Methods, book 5, chap. B2, 34 p., https://doi.org/10.3133/tm5B2.

California Department of Water Resources, 2019, Statewide cropping layer 2014, accessed April 5, 2019, at https://gis.water.ca.gov/app/CADWRLandUseViewer/.

California Water Boards, 2014, Occurrence of blue green algae toxins in central coast streams: Surface Water Ambient Monitoring Program, Central Coast Region Report, https://www.waterboards.ca.gov/water_issues/programs/ swamp/docs/reglrpts/rb3_bcalgae_report.pdf.

Charles, D.F., Knowles, C., and Davis, R.S., eds., 2002, Protocols for the analysis of algal samples collected as part of the U.S. Geological Survey National Water-Quality Assessment Program: Philadelphia, Pa., The Academy of Natural Sciences Report No. 02-06, 124 p., http://diatom.ansp.org/nawqa/Protocols.aspx.

Coles, J.F., McMahon, G., Bell, A.H., Brown, L.R., Fitzpatrick, F.A., Scudder Eikenberry, B.C., Woodside, M.D., Cuffney, T.F., Bryant, W.L., Jr., Cappiella, K., Fraley-McNeal, L., and Stack, W.P., 2012, Effects of urban development on stream ecosystems in nine metropolitan study areas across the United States: U.S. Geological Survey Circular 1373, 138 p., https://doi.org/10.3133/cir1373.

Coles, J.F., Riva-Murray, K., Van Metre, P.C., Button, D.T., Bell, A.H., Qi, S.L., Journey, C.A., and Sheibley, R.W., 2019, Design and methods of the U.S. Geological Survey Northeast Stream Quality Assessment (NESQA), 2016: U.S. Geological Survey Open-File Report 2018-1183, 46 p., https://doi.org/10.3133/ofr20181183.

Coplen, T.B., Qi, H., Révész, K., Casciotti, K., and Hannon, J.E., 2012, Determination of the $\delta^{15} \mathrm{~N}$ and $\delta^{18} \mathrm{O}$ of nitrate in water; RSIL lab code 2900, chap. 17 of Stable isotope-ratio methods, sec. C of Révész, K, and Coplen, T.B., eds., Methods of the Reston Stable Isotope Laboratory [slightly revised from ver. 1.0 released in 2006]: U.S. Geological Survey Techniques and Methods, book 10, chap. 17C, 35 p., https://pubs.usgs.gov/tm/2006/tm10c17/.

Cuffney, T.F., Kashuba, R., Qian, S.S., Alameddine, I., Cha, Y.K., Lee, B., Coles, J.F., and McMahon, G., 2011, Multilevel regression models describing regional patterns of invertebrate and algal responses to urbanization across the USA: Journal of the North American Benthological Society, v. 30, no. 3, p. 797-819, https://doi.org/10.1899/10-140.1.
Culp, J.M., Yates, A.G., Armanini, D.G., and Baird, D.J., 2017, Establishing cause-effect relationships in multistressor environments, chap. 40, in Lamberti, G.A., and Hauer, F.R., eds., Methods in Stream Ecology (3d ed.), Volume 2-Ecosystem Function: Elsevier Inc., p. 335-357, https://works.bepress.com/adam-yates/9/.

Davis, B.E., 2005, A guide to the proper selection and use of federally approved sediment and water-quality samplers: U.S. Geological Survey Open-File Report 2005-1087, 20 p., https://doi.org/10.3133/ofr20051087.

DeWild, J.F., Olsen, M.L., and Olund, S.D., 2002, Determination of methyl mercury by aqueous phase ethylation, followed by gas chromatographic separation with cold vapor atomic fluorescence detection: U.S. Geological Survey Open-File Report 2001-445, 14 p., https://doi.org/10.3133/ofr2001445.

DeWild, J.F., Olund, S.D., Olson, M.L., and Tate, M.T., 2004, Methods for the preparation and analysis of soilds and suspended solids for methylmercury: U.S. Geological Survey Techniques and Methods, book 5, chap. 7, sec. A., https://pubs.usgs.gov/tm/2005/tm5A7/.

Dunham, J., Chandler, G., Rieman, B., and Martin, D., 2005, Measuring stream temperature with digital data loggers - A user's guide: U.S. Department of Agriculture, Forest Service, Rocky Mountain Research Station, General Technical Report RMRS-GTR-150WWW, 16 p., accessed December 19, 2014, at https://www.fs.fed.us/rm/pubs/rmrs_gtr150.pdf.

Dupré, D.H., Scott, J.C., Clark, M.L., Canova, M.G., and Stoker, Y.E., 2013, User's manual for the National Water Information System of the U.S. Geological SurveyWater-Quality System, Version 5.0: U.S. Geological Survey Open-File Report 2013-1054, 730 p., https://doi.org/10.3133/ofr20131054.

Environmental Systems Research Institute, 2009, World Terrain Base; Esri basemap, accessed January 1, 2017, at https://www.arcgis.com/home/item.html?id= c61ad8ab017d49e1a82f580ee1298931.

Environmental Systems Research Institute, GEBCO, NOAA, National Geographic, Garmin, and HERE, 2014: World Ocean Base; Esri basemap, accessed July 1, 2019, at https://www.arcgis.com/home/item.html?id= 1e126e7520f9466c9ca28b8f28b5e500.

Falcone, J.A., 2015, U.S. conterminous wall-to-wall anthropogenic land use trends (NWALT), 1974-2012: U.S. Geological Survey Data Series 948, 33 p., accessed April 17, 2017, at https://doi.org/10.3133/ds948. 
Fischer, J.M., Phillips, P.J., Reilly, T.J., Focazio, M.J., Loftin, K.A., Benzel, W.M., Jones, D.K., Smalling, K.L., Fisher, S.C., Fisher, I.J., Iwanowicz, L.R., Romanok, K.M., Jenkins, D., Bowers, L., Boehlke, A., Foreman, W.T., Deetz, A.C., Carper, L.G., Imbrigiotta, T.E., and Birdwell, J., 2015, Estuarine bed-sediment-quality data collected in New Jersey and New York after Hurricane Sandy, 2013: U.S. Geological Survey Data Series 905, 42 p., plus CD-ROM, https://doi.org/10.3133/ds905.

Fishman, M.J., ed., 1993, Methods of analysis by the U.S. Geological Survey National Water Quality Laboratory-Determination of inorganic and organic constituents in water and fluvial sediments, U.S. Geological Survey Open-File Report 93-125, 217 p., https://doi.org/10.3133/ofr93125.

Fishman, M.J., and Friedman, L.C., 1989, Methods for determination of inorganic substances in water and fluvial sediments: U.S. Geological Survey Techniques of Water-Resources Investigations, book 5, chap. A1, 545 p., https://doi.org/10.3133/twri05A1.

Fitzpatrick, F.A., Waite, I.R., D’Arconte, P.J., Meador, M.R., Maupin, M.A., and Gurtz, M.E., 1998, Revised methods for characterizing stream habitat in the National Water-Quality Assessment Program: U.S. Geological Survey Water-Resources Investigations Report 98-4052, 67 p., https://doi.org/10.3133/wri984052.

Foreman, W.T., Gray, J.L., ReVello, R.C., Lindley, C.E., Losche, S.A., and Barber, L.B., 2012, Determination of steroid hormones and related compounds in filtered and unfiltered water by solid-phase extraction, derivatization, and gas chromatography with tandem mass spectrometry: U.S. Geological Survey Techniques and Methods, book 5, chap. B9, 118 p., https://doi.org/10.3133/tm5B9.

Furlong, E.T., Werner, S.L., Anderson, B.D., and Cahill, J.D., 2008, Determination of human-health pharmaceuticals in filtered water by chemically modified styrene-divinylbenzene resin-based solid-phase extraction and high-performance liquid chromatography/mass spectrometry: U.S. Geological Survey Techniques and Methods, book 5, chap. B5, 56 p., https://doi.org/10.3133/tm5B5.

Furlong, E.T., Noriega, M.C., Kanagy, C.J., Kanagy, L.K., Coffey, L.J., and Burkhardt, M.R., 2014, Determination of human-use pharmaceuticals in filtered water by direct aqueous injection-high-performance liquid chromatography/tandem mass spectrometry: U.S. Geological Survey Techniques and Methods, book 5, chap. B10, 49 p., accessed April 3, 2015, at https://doi.org/10.3133/tm5B10.
Garrett, J.D., Frey, J.W., Van Metre, P.C., Journey, C.A., Nakagaki, N., Button, D.T., and Nowell, L.H., 2017, Design and methods of the midwest stream quality assessment (MSQA), 2013: U.S. Geological Survey Open-File Report 2017-1073, 59 p., https://doi.org/10.3133/ofr20171073.

Gellis, A.C., Fuller, C.C., Van Metre, P., Filstrup, C.T., Tomer, M.D., Cole, K.J., and Sabitov, T.Y., 2018, Combining sediment fingerprinting with age-dating sediment using fallout radionuclides for an agricultural stream, Walnut Creek, Iowa, USA: Journal of Soils and Sediments, v. 19, p. 3374-3396, https://doi.org/10.1007/s11368-018-2168-z.

Gibble, C.M., and Kudela, R.M., 2014, Detection of persistent microcystin toxins at the land-sea interface in Monterey Bay, California: Harmful Algae, v. 39, p. 146-153, https://doi.org/10.1016/j.hal.2014.07.004.

Gregory, M.B., and Calhoun, D.L., 2007, Physical, chemical, and biological responses of streams to increasing watershed urbanization in the Piedmont ecoregion of Georgia and Alabama, 2003, chap. B of Effects of urbanization on stream ecosystems in six metropolitan areas of the United States: U.S. Geological Survey Scientific Investigations Report 2006-5101-B, 104 p., https://doi.org/10.3133/sir20065101B.

Griffith, G.E., Omernik, J.M., Smith, D.W., Cook, T.D., Tallyn, E., Moseley, K., and Johnson, C.B., 2016, Ecoregions of California: U.S. Geological Survey Open-File Report 2016-1021, poster with map, scale 1:1,100,000, https://doi.org/10.3133/ofr20161021.

Guy, H.P., 1969, Laboratory theory and methods for sediment analysis: U.S. Geological Survey Techniques of Water-Resources Investigations, book 5, chap. C1, 58 p., https://doi.org/10.3133/twri05C1.

Herlihy, A.T., Hughes, R.M., and Sifneos, R.C., 2006, National clusters of fish species assemblages in the conterminous United States and their relationship to existing landscape classification schemes, in Hughes, R.M., Wang, L., and Seelbach, P.W., eds, Landscape influences on stream habitats and biological assemblages: Proceedings of the Symposium on Influences of Landscape on Stream Habitat and Biological Communities held in Madison, Wisconsin, USA, August 25-26, 2004, Bethesda, MD, American Fisheries Society, p. 87-112.

Herlihy, A.T., Paulsen, S.G., Van Sickle, J., Stoddard, J.L., Hawkins, C.P., and Yuan, L.L., 2008, Striving for consistency in a national assessment-The challenges of applying a reference-condition approach at a continental scale: Journal of the North American Benthological Society, v. 27, no. 4, p. 860-877, https://doi.org/10.1899/08-081.1. 
Hladik, M.L., and McWayne, M.M., 2012, Methods of analysis-Determination of pesticides in sediment using gas chromatography/mass spectrometry: U.S. Geological Survey Techniques and Methods, book 5, chap. C3, 18 p., https://doi.org/10.3133/tm5C3.

Hladik, M.L., Vandever, M., and Smalling, K.L., 2016, Exposure of native bees foraging in an agricultural landscape to current-use pesticides: Science of the Total Environment, v. 542, Part A, p. 469-477, https://doi.org/10.1016/j.scitotenv.2015.10.077.

Homer, C.G., Dewitz, J.A., Yang, L., Jin, S., Danielson, P., Xian, G., Coulston, J., Herold, N.D., Wickham, J.D., and Megown, K., 2015, Completion of the 2011 National Land Cover Database for the conterminous United StatesRepresenting a decade of land cover change information: American Society for Photogrammetry and Remote Sensing, v. 81, p. 345-354, https://cfpub.epa.gov/si/si public_record_report.cfm?Lab $=$ NERL\&dirEntryId $=309950$.

Journey, C.A., Van Metre, P.C., Bell, A.H., Garrett, J.D., Button, D.T., Nakagaki, N., Qi, S.L., and Bradley, P.M., 2015, Design and methods of the southeast stream quality assessment (SESQA), 2014: U.S. Geological Survey Open-File Report 2015-1095, 46 p., https://doi.org/10.3133/ofr20151095.

Kemble, N.E., Hardesty, D.K., Ingersoll, C.G., Kunz, J.L., Sibley, P.K., Calhoun, D.L., Gilliom, R.J., Kuivila, K.M., Nowell, L.H., and Moran, P.W., 2013, Contaminants in stream sediments from seven United States metropolitan areas-Part II-Sediment toxicity to the amphipod Hyalella azteca and the midge Chironomus dilutus: Archives of Environmental Contamination and Toxicology, v. 64, no. 1, p. 52-64, https://doi.org/10.1007/s00244-012-9815-y.

Klemm, D.J., Blocksom, K.A., Fulk, F.A., Herlihy, A.T., Hughes, R.M., Kaufmann, P.R., Peck, D.V., Stoddard, J.L., Thoeny, W.T., Griffith, M.B., and Davis, W.S., 2003, Development and evaluation of a macroinvertebrate biotic integrity index (MBII) for regionally assessing Mid-Atlantic Highlands streams: Environmental Management, v. 31, no. 5, p. 656-669, https://doi.org/10.1007/s00267-002-2945-7.

Knott, J.M., Glysson, G.D., Malo, B.A., and Schroeder, L.J., 1993, Quality assurance plan for the collection and processing of sediment data by the U.S. Geological Survey, Water Resources Division: U.S. Geological Survey Open-File Report 92-499, 18 p., accessed November 17, 2010, at https://doi.org/10.3133/ofr92499.

Land IQ, LLC, 2017, Land Use - 2014 - Land IQ:

Prepared by Land IQ, LLC for the California Department of Water Resources and other resource agencies, accessed April 5, 2019, at https://gis.water.ca.gov/app/CADWRLandUseViewer.
Leahy, P.P., Rosenshein, J.S., and Knopman, D.S., 1990, Implementation plan for the National Water-Quality Assessment Program: U.S. Geological Survey Open-File Report 90-174, 10 p., https://doi.org/10.3133/ofr90174.

Lenat, D.R., and Crawford, J.K., 1994, Effects of land use on water quality and aquatic biota of three North Carolina Piedmont streams: Hydrobiologia, v. 294, no. 3, p. 185-199, https://doi.org/10.1007/BF00021291.

Lewis, M.E., and Brigham, M.E., 2004, Low level mercury: U.S. Geological Survey Techniques of Water-Resources Investigations, book 9, chap. A5, sec. 5.6.4.B, 26 p., accessed March 16, 2007, at https://water.usgs.gov/owq/ FieldManual/chapter5/pdf/5.6.4.B_v1.0.pdf.

Loftin, K.A., Clark, J.M., Journey, C.A., Kolpin, D.W., Van Metre, P.C., Carlisle, D., and Bradley, P.M., 2016, Spatial and temporal variation in microcystin occurrence in wadeable streams in the southeastern United States: Environmental Toxicology and Chemistry, v. 35, no. 9, p. 2281-2287, accessed November 2016 at https://doi.org/10.1002/etc.3391.

Mahler, B.J., Van Metre, P.C., Wilson, J.T., Musgrove, M., Zaugg, S.D., and Burkhardt, M.R., 2009, Fipronil and its degradates in indoor and outdoor dust: Environmental Science \& Technology, v. 43, no. 15, p. 5665-5670, https://doi.org/10.1021/es901292a.

Mahler, B.J., Van Metre, P.C., Burley, T.E., Loftin, K.A., Meter, M.T., and Nowell, L.H., 2017, Similarities and differences in occurrence and temporal fluctuations in glyphosate and atrazine in small Midwestern streams (USA) during the 2013 growing season: Science of the Total Environment, v. 579, p. 149-158, accessed May 2017 at https://doi.org/10.1016/j.scitotenv.2016.10.236.

McWhirter, K.J., 2020, Technical description of small volume autonomous water sampler (SVAWS), supporting information, Appendix I, in Norman, J.E. Nowell, L.H., Van Metre, P.C., Mahler, B.J., Sandstrom, M.W., Corbin, M.A., Qian, Y., Pankow, J.F., Luo, W., Fitzgerald, N.B., Asher, W.A., McWhirter, K.J., Daily stream samples reveal highly complex pesticide occurrence and potential toxicity to aquatic life: Science of the Total Environment, v. 715, 13 p., https://doi.org/10.1016/j.scitotenv.2020.136795.

Meyer, M.T., Loftin, K.A., Lee, E.A., Hinshaw, G.H., Dietze, J.E., and Scribner, E.A., 2009, Determination of glyphosate, its degradation product aminomethylphosphonic acid, and glufosinate, in water by isotope dilution and online solid-phase extraction and liquid chromatography/ tandem mass spectrometry: U.S. Geological Survey Techniques and Methods, book 5, chap. A10, 32 p., https://doi.org/10.3133/tm5A10. 
Moran, P.W., Nowell, L.H., Kemble, N.E., Mahler, B.J., Waite, I.R., and Van Metre, P.C., 2017, Influence of sediment chemistry and sediment toxicity on macroinvertebrate communities across 99 wadable streams of the Midwestern USA: Science of the Total Environment, v. 599-600, p. 1469-1478, accessed December 1, 2017, at https://doi.org/10.1016/j.scitotenv.2017.05.035.

Moulton, S.R., II, Carter, J.L., Grotheer, S.A., Cuffney, T.F., and Short, T.M., 2000, Methods of analysis by the U.S. Geological Survey National Water Quality Laboratory-Processing, taxonomy, and quality control of benthic macroinvertebrate samples: U.S. Geological Survey Open-File Report 2000-212, 49 p., https://doi.org/10.3133/ofr00212.

Moulton, S.R., II, Kennen, J.G., Goldstein, R.M., and Hambrook, J.A., 2002, Revised protocols for sampling algal, invertebrate, and fish communities as part of the National Water-Quality Assessment Program: U.S. Geological Survey Open-File Report 02-150, 75 p., accessed April 3, 2015, at https://doi.org/10.3133/ofr2002150.

Mueller, D.K., Martin, J.D., and Lopes, T.J., 1997, Quality-control design for surface-water sampling in the National Water-Quality Assessment Program: U.S. Geological Survey Open-File Report 97-223, 17 p., https://doi.org/10.3133/ofr97223.

Nagy, R.C., Lockaby, B.G., Helms, B., Kalin, L., and Stoeckel, D., 2011, Water resources and land use and cover in a humid region-The southeastern United States: Journal of Environmental Quality, v. 40, no. 3, p. 867-878, accessed April 3, 2015, at https://doi.org/10.2134/jeq2010.0365.

Norman, J.E., Kuivila, K.M., and Nowell, L.H., 2012, Prioritizing pesticide compounds for analytical methods development: U.S. Geological Survey Scientific Investigations Report 2012-5045, 74 p., https://doi.org/10.3133/sir20125045.

Ode, P.R., Fetscher, A.E., and Busse, L.B., 2016, Standard operating procedures (SOP) for the collection of field data for bioassessments of California Wadeable Streams - Benthic macroinvertebrates, algae, and physical habitat: California State Water Resources Control Board Surface Water Ambient Monitoring Program (SWAMP) Bioassessment SOP 004, 80 p., https://www.waterboards.ca.gov/water_issues/programs/ swamp/bioassessment/sops.html.
Ode, P.R., Kincaid, T.M., Fleming, T., and Rehn, A.C., 2011, Ecological condition assessments of California's perennial wadeable streams - Highlights from the surface water ambient monitoring program's Perennial Streams Assessment (PSA) (2000-2007), accessed June 1, 2016, at https://www.waterboards.ca.gov/water_issues/programs/ swamp/docs/reports/psa_smmry_rpt.pdf.

O’Dell, J.W., 1993, Method 365.1, Revision 2.0Determination of phosphorus by semi-automated colorimetry: Cincinnati, Ohio, U.S. Environmental Protection Agency, Office of Research and Development, 17 p., https://www.epa.gov/sites/production/files/2015-08/ documents/method_365-1_1993.pdf.

Omernik, J.M., and Griffith, G.E., 2014, Ecoregions of the conterminous United States-Evolution of a hierarchical spatial framework: Environmental Management, v. 54, no. 6, p. 1249-1266, https://doi.org/10.1007/s00267-014-0364-1.

Onset Computer Corporation, 2012, HOBO ${ }^{\circledR}$ Water Temp Pro v2 (U22-001) manual-User manual 10366-G: Onset Computer Corporation user manual, 3 p., accessed December 13, 2013, at https://www.onsetcomp.com/files/ manual_pdfs/10366-G-MAN-U22-001.pdf.

Onset Computer Corporation, 2014, $\mathrm{HOBO}^{\circledR} \mathrm{U} 20$ Water Level Logger (U20-001-0x and U20-001-0x-Ti) manual: Onset Computer Corporation user manual, 7 p., accessed December 31, 2014, at https://www.onsetcomp.com/files/ manual_pdfs/12315-F-MAN-U20.pdf.

Patton, C.J., and Kryskalla, J.R., 2011, Colorimetric determination of nitrate plus nitrite in water by enzymatic reduction, automated discrete analyzer methods: U.S. Geological Survey Techniques and Methods, book 5, chap. B8, 34 p.,https://doi.org/10.3133/tm5B8.

Phillips, J.M., Russell, M.A., and Walling, D.E., 2000, Time-integrated sampling of fluvial suspended sediment-A simple methodology for small catchments: Hydrological Processes, v. 14, no. 14, p. 2589-2602, https://doi.org/10.1002/10991085(20001015)14:14\%3C2589::AIDHYP94\%3E3.0.CO;2-D.

Qi, S.L. and Nakagaki, N., 2020, Selected environmental characteristics of sampled sites for the U.S. Geological Survey regional stream-quality assessment: U.S. Geological Survey data release, https://doi.org/10.5066/P962N215.

Radtke, D.B., 2005, Bottom-material samples: U.S. Geological Survey Techniques of Water-Resources Investigations, book 9, chap. A8, variously paged, accessed February 22, 2007, at https://doi.org/10.3133/twri09A8. 
Rehn, A.C., Mazor, R.D., and Ode, P.R., 2015, The California stream condition index (CSCI) - A new statewide biological scoring tool for assessing the health of freshwater streams: Surface Water Ambient Monitoring Program Technical Memorandum SWAMP-TM-2015-0002, https://www.waterboards.ca.gov/water_issues/programs/ swamp/bioassessment/docs/csci_tech_memo.pdf.

Sabater, S., Guasch, H., Ricart, M., Romaní, A., Vidal, G., Klünder, C., and Schmitt-Jansen, M., 2007, Monitoring the effect of chemicals on biological communities - The biofilm as an interface: Analytical and Bioanalytical Chemistry, v. 387, p. 1425-1434, https://doi.org/10.1007/s00216-006-1051-8.

Sandstrom, M.W., Kanagy, L.K., Anderson, C.A., and Kanagy, C.J., 2016, Determination of pesticides and pesticide degradates in filtered water by direct aqueous-injection liquid chromatography-tandem mass spectrometry: U.S. Geological Survey Techniques and Methods, book 5, chap B11, 54 p., https://doi.org/10.3133/tm5B11.

Sauer, V.B., and Turnipseed, D.P., 2010, Stage measurement at gaging stations: U.S. Geological Survey Techniques and Methods, book 3, chap. A7, 45 p., https://doi.org/10.3133/tm3A7.

Sheibley, R.W., Morace, J.L., Journey, C.A., Van Metre, P.C., Bell, A.H., Nakagaki, N., Button, D.T., and Qi, S.L., 2017, Design and methods of the Pacific Northwest stream quality assessment (PNSQA), 2015: U.S. Geological Survey Open-File Report 2017-1103, 46 p. https://doi.org/10.3133/ofr20171103.

Shelton, L.R., and Capel, P.D., 1994, Guidelines for collecting and processing samples of stream bed sediment for analysis of trace elements and organic contaminants for the National Water-Quality Assessment Program: U.S. Geological Survey Open-File Report 94-458, 20 p., https://doi.org/10.3133/ofr94458.

Smith, D.B., Cannon, W.F., Woodruff, L.G., Solano, F., Kilburn, J.E., and Fey, D.L., 2013, Geochemical and mineralogical data for soils of the conterminous United States: U.S. Geological Survey Data Series 801, 19 p., accessed July 1, 2015, at https://doi.org/10.3133/ds801.

Spaulding, S.A., Lubinski, D.J., and Potapova, M., 2010, Diatoms of the United States: University of Colorado website, accessed March 1, 2017, at http://westerndiatoms.colorado.edu.

Townsend, C.R., Uhlmann, S.S., and Matthaei, C.D., 2008, Individual and combined responses of stream ecosystems to multiple stressors: Journal of Applied Ecology, v. 45, no. 6, p. 1810-1819, https://doi.org/10.1111/j.1365-2664.2008.01548.x.
U.S. Environmental Protection Agency, 1996, Method 1669-Sampling ambient water for trace metals at EPA water quality criteria levels: Washington, D.C., U.S. Environmental Protection Agency, Office of Water, Engineering and Analysis Division, 37 p., accessed March 12, 2011, at https://www.epa.gov/sites/production/ files/2015-10/documents/method_1669_1996.pdf.

U.S. Environmental Protection Agency, 2000, Methods for measuring the toxicity and bioaccumulation of sediment-associated contaminants with freshwater invertebrates (2d ed., March 2000): Duluth, Minn., and Washington, D.C., U.S. Environmental Protection Agency Office of Research and Development and Office of Water, EPA 600/R-99/064, 192 p.

U.S. Environmental Protection Agency, 2002, Method 1631, Revision E-Mercury in water by oxidation, purge and trap, and cold vapor atomic fluorescence spectrometry: Washington, D.C., U.S. Environmental Protection Agency, Office of Water, EPA-821-R-02-019, 38 p., https://www.epa.gov/sites/production/files/2015-08/ documents/method_1631e_2002.pdf.

U.S. Environmental Protection Agency, 2006, Wadeable streams assessment-A collaborative survey of the Nation's streams: Washington, D.C., U.S. Environmental Protection Agency, Office of Research and Development and Office of Water, EPA 841-B-06-002, 98 p., https://www.epa.gov/ sites/production/files/2014-10/documents/2007_5_16_ streamsurvey_wsa_assessment_may2007.pdf.

U.S. Environmental Protection Agency, 2013, California integrated assessment of watershed health: U.S. Environmental Protection Agency, EPA 841-R-14-003, 110 p., https:/www.epa.gov/sites/production/files/2015-11/ documents/ca_hw_report_111213_0.pdf.

U.S. Environmental Protection Agency, 2014, Method 8270D_-Semivolatile organic compounds by gas chromatography/mass spectrometry: U.S. Environmental Protection Agency, SW-846 Update V, 71 p., https://19january2017snapshot.epa.gov/sites/production/ files/2015-12/documents/8270d.pdf.

U.S. Environmental Protection Agency, 2016, National rivers and streams assessment 2008-2009-A collaborative survey: Washington, D.C., U.S. Environmental Protection Agency, Office of Water and Office of Research and Development, EPA/841/R-16/007, https:/www.epa.gov/ sites/production/files/2016-03/documents/nrsa_0809_ march_2_final.pdf.

U.S. Environmental Protection Agency, 2019, National summary of state information-Summary of water quality assessments for each waterbody type, accessed December 12, 2019, at https://ofmpub.epa.gov/waters10/ attains_nation_cy.control\#total_assessed_waters. 
U.S. Environmental Protection Agency and U.S. Geological Survey, 2012, National Hydrography Dataset PlusNHDPlus, version 2, edition 2.10: U.S. Environmental Protection Agency and U.S. Geological Survey geospatial data, accessed January 21, 2015, at http://www.horizon-systems.com/NHDPlus/index.php.

U.S. Geological Survey, 2006, Collection of water samples (ver. 2.0, September 2006): U.S. Geological Survey Techniques of Water-Resources Investigations, book 9, chap. A4, 166 p., accessed October 24, 2014, at https://doi.org/10.3133/twri09A4.

U.S. Geological Survey, 2014, NLCD 2011 land coverNational geospatial data asset (NGDA) land use land cover (2011 ed., amended October 10, 2014): U.S. Geological Survey dataset, accessed February 1, 2015, at http://www.mrlc.gov.

U.S. Geological Survey, 2016, BioData-Aquatic bioassessment data for the Nation: U.S. Geological Survey database, accessed January 1, 2017, at https://doi.org/10.5066/F77W698B.

U.S. Geological Survey, variously dated, National field manual for the collection of water-quality data: U.S. Geological Survey Techniques of Water-Resources Investigations, book 9, chaps. A1A10, variously paged, accessed December 3, 2014, at https://water.usgs.gov/owq/FieldManual/.

Van Metre, P.C., Wilson, J.T., Fuller, C.C., Callender, E., and Mahler, B.J., 2004, Collection, analysis, and age-dating of sediment cores from 56 U.S. lakes and reservoirs sampled by the U.S. Geological Survey, 1992-2001: U.S. Geological Survey Scientific Investigations Report 2004-5184, 187 p., https://doi.org/10.3133/sir20045184.

Van Metre, P.C., Alvarez, D., Mahler, B.J., Nowell, L.H., Sandstrom, M.W., and Moran, P.W., 2017a, Complex mixtures of pesticides in Midwest U.S. streams indicated by POCIS time-integrating samplers: Environmental Pollution, v. 220, Part A, p. 431-440, accessed February 1, 2017, at https://doi.org/10.1016/j.envpol.2016.09.085.

Van Metre, P.C., Egler, A.L., and May, J.T., 2017b, The California stream quality assessment: U.S. Geological Survey Fact Sheet 2017-3014, 2 p., https://doi.org/10.3133/fs20173014.
Wagner, R.J., Moran, P.W., Zaugg, S.D., Sevigny, J.M., and Pope, J.M., 2014, Contaminants of emerging concern in the lower Stillaguamish River Basin, Washington, 2008-11 (ver. 2.0, June 2016): U.S. Geological Survey Open-File Report 2014-1028, 14 p., accessed August 1, 2016, at https://doi.org/10.3133/ofr20141028.

Waite, I.R., Herlihy, A.T., Larsen, D.P., and Klemm, D.J., 2000, Comparing strengths of geographic and nongeographic classifications of stream benthic macroinvertebrates in the Mid-Atlantic Highlands, USA: Journal of the North American Benthological Society, v. 19, no. 3, p. 429-441, https://doi.org/10.2307/1468105.

Wiken, E., Jiménez Nava, F., and Griffith, G., 2011, North American terrestrial ecoregions-Level III: Montreal, Canada, Commission for Environmental Cooperation, 149 p., http://www3.cec.org/islandora/en/item/10415-northamerican-terrestrial-ecoregionslevel-iii.

Wilde, F.D., ed., 2008, Field measurements: U.S. Geological Survey Techniques of Water-Resources Investigations, book 9, chap. A6, accessed December 3, 2014, at https://pubs.water.usgs.gov/twri9A.

Wilde, F.D., Radtke, D.B., Gibs, J., and Iwatsubo, R.T., eds., 2009, Processing of water samples (ver. 2.2, September 2004 with updates through 2009): U.S. Geological Survey Techniques of Water-Resources Investigations, book 9, chap. A5, accessed October 24, 2014, at https://pubs.water.usgs.gov/twri9A5/.

Woodard, M.E., Slusark, J., and Ode, P.R., 2012, Standard operating procedures for laboratory processing and identification of benthic macroinvertebrates in California: California State Water Resources Control Board, Surface Water Ambient Monitoring Program (SWAMP) Bioassessment SOP 003, 37 p., https://www.waterboards.ca.gov/water_issues/programs/ swamp/docs/bmi_lab_sop_final.pdf.

Zaugg, S.D., Smith, S.G., and Schroeder, M.P., 2006, Determination of wastewater compounds in whole water by continuous liquid-liquid extraction and capillary-column gas chromatography/mass spectrometry: U.S. Geological Survey Techniques and Methods, book 5, chap. B4, 30 p., https://doi.org/10.3133/tm5B4. 


\section{Appendix 1. Description of the Sampling Timelines, Matrix, Collection, and Processing for Water, Sediment, and Ecological Samples}

Table 1.1. Sampling matrix for the 85 sites sampled as part of the U.S. Geological Survey California Stream Quality Assessment in 2017 is provided as a downloadable Excel file available at https://doi.org/10.3133/sir20201023.

Table 1.2. Onset Computer Corporation specifications for the HOBO Water Temp Pro v2 U22 and U20 water-level loggers used to monitor continuous water temperature and water level, respectively, at selected stream sites as part of the California Stream Ouality Assessment study of the U.S. Geological Survey National Water-Quality Assessment Project in 2017.

$\left[{ }^{\circ} \mathrm{C}\right.$, degree Celsius; ${ }^{\circ} \mathrm{F}$, degree Fahrenheit; \pm , plus or minus; $\mathrm{m} / \mathrm{s}$, meter per second; $\mathrm{kPa}$, kilopascal; psi, pound per square inch; $\mathrm{m}$, meter; ft. foot; FS, full scale pressure; cm, centimeter; $<$, less than]

\begin{tabular}{|c|c|}
\hline Specifications & Range \\
\hline \multicolumn{2}{|c|}{ HOB0 ${ }^{\circledR}$ Water Temp Pro v2 U22 Logger } \\
\hline Operation range & $\begin{array}{l}-40 \text { to } 70^{\circ} \mathrm{C}\left(-40 \text { to } 158^{\circ} \mathrm{F}\right) \text { in air; maximum sustained tempera- } \\
\text { ture of } 50^{\circ} \mathrm{C}\left(122^{\circ} \mathrm{F}\right) \text { in water }\end{array}$ \\
\hline Accuracy & $\pm 0.21{ }^{\circ} \mathrm{C}$ from 0 to $50^{\circ} \mathrm{C}\left( \pm 0.38^{\circ} \mathrm{F}\right.$ from 32 to $\left.122^{\circ} \mathrm{F}\right)$ \\
\hline Resolution & $0.02{ }^{\circ} \mathrm{C}$ at $25^{\circ} \mathrm{C}\left(0.04^{\circ} \mathrm{F}\right.$ at $\left.77^{\circ} \mathrm{F}\right)$ \\
\hline \multicolumn{2}{|c|}{ HOBO ${ }^{\circledR}$ U20-001-04 Water Level Logger } \\
\hline Operation range & $\begin{array}{l}0 \text { to } 145 \mathrm{kPa}(0 \text { to } 21 \mathrm{psi}) \text {; approximately } 0 \text { to } 4 \mathrm{~m}(0 \text { to } 13 \mathrm{ft}) \text { of } \\
\text { water depth at sea level }\end{array}$ \\
\hline Accuracy & \pm 0.75 percent $F S, 0.3 \mathrm{~cm}(0.01 \mathrm{ft})$ water \\
\hline
\end{tabular}


Table 1.3. Description of the data collection and processing steps for water samples collected during the U.S. Geological Survey California Stream Quality Assessment study in 2017.

[4.5 N H2SO4, 4.5 normal sulfuric acid; 7.5N HNO3, 7.5 normal nitric acid; AR, acid-rinsed; DH-81 and DH-95, sampling devices; DOC, dissolved organic carbon; EWI, equal-width increment sampling protocol; ft, foot; ft/s, foot per second; HCl, hydrochloric acid; Hg, mercury; L, liter; LS, Kansas; m, meter; $\mathrm{mL}$, milliter; poly, polyethylene bottle; TX, Texas; UVA, ultraviolet absorbance; - , not applicable; < less than; >, greater than. Sample type labels are designated by the National Water Quality Laboratory: BGC, baked, amber glass wide-mouth jar, chilled; FA, filtered, acidified cations; FCC, filtered, chilled nutrients; FU, filtered, unpreserved anions; GCC, baked, amber glass bottle, chilled; GLY2, glyphosate by U.S. Geological Survey Kansas Water Science Center; IMMGLY, glyphosate by immunoassay; OWI, organic wastewater indicators; PEST, pesticide; PHARM, pharmaceutical; RU, raw, unpreserved physical properties; SSC, suspended sediment concentration; WCA, whole, chilled, acidified nutrients]

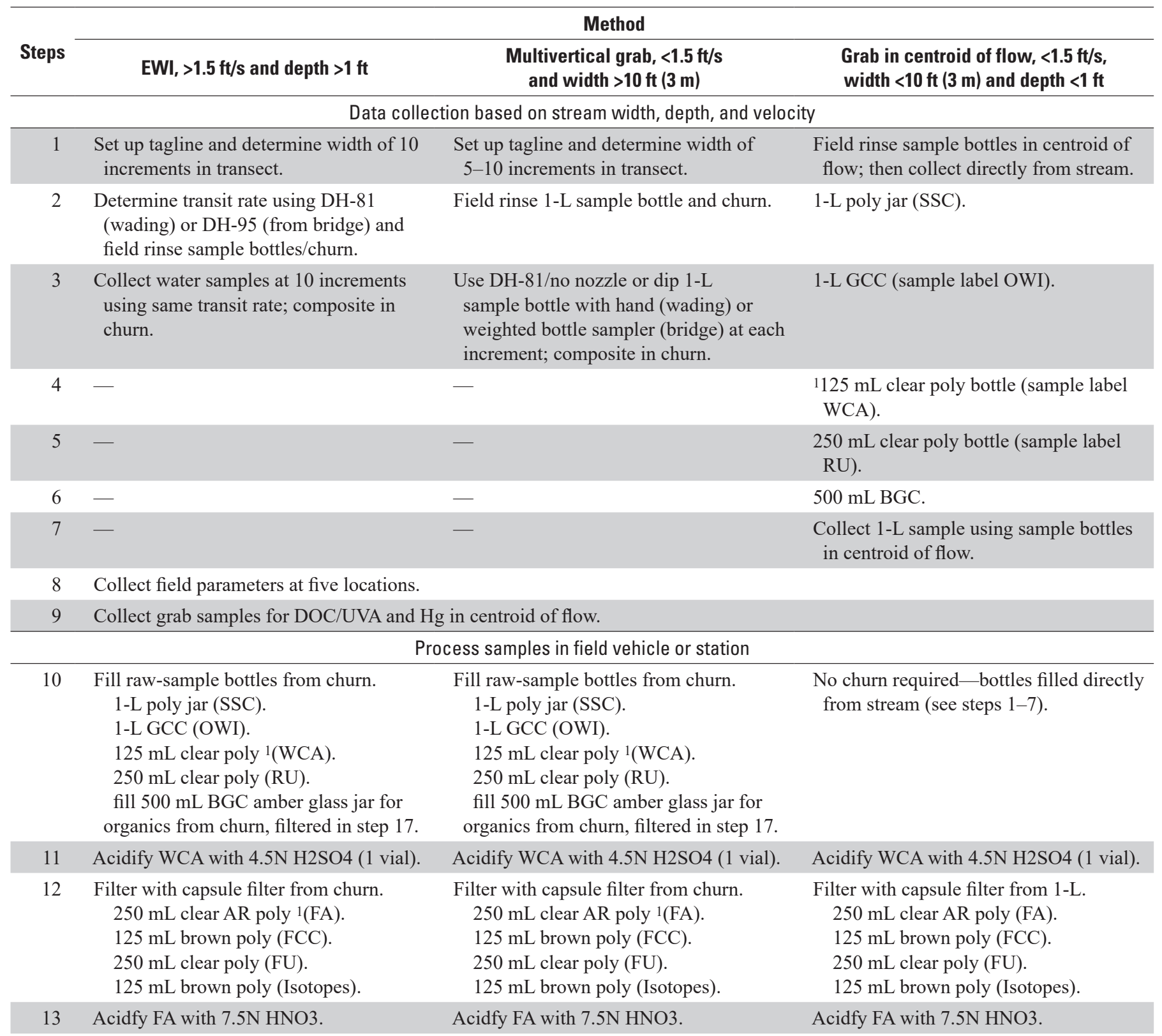


Table 1.3. Description of the data collection and processing steps for water samples collected during the U.S. Geological Survey California Stream Quality Assessment study in 2017.-Continued

[4.5 N H2SO4, 4.5 normal sulfuric acid; 7.5N HNO3, 7.5 normal nitric acid; AR, acid-rinsed; DH-81 and DH-95, sampling devices; DOC, dissolved organic carbon; EWI, equal-width increment sampling protocol; ft, foot; ft/s, foot per second; HCl, hydrochloric acid; Hg, mercury; L, liter; LS, Kansas; m, meter; mL, milliter; poly, polyethylene bottle; TX, Texas; UVA, ultraviolet absorbance; - , not applicable; < less than; >, greater than. Sample type labels are designated by the National Water Quality Laboratory: BGC, baked, amber glass wide-mouth jar, chilled; FA, filtered, acidified cations; FCC, filtered, chilled nutrients; FU, filtered, unpreserved anions; GCC, baked, amber glass bottle, chilled; GLY2, glyphosate by U.S. Geological Survey Kansas Water Science Center; IMMGLY, glyphosate by immunoassay; OWI, organic wastewater indicators; PEST, pesticide; PHARM, pharmaceutical; RU, raw, unpreserved physical properties; SSC, suspended sediment concentration; WCA, whole, chilled, acidified nutrients]

\begin{tabular}{|c|c|c|c|}
\hline \multirow[b]{2}{*}{ Steps } & \multicolumn{3}{|c|}{ Method } \\
\hline & $\mathrm{EWI},>1.5 \mathrm{ft} / \mathrm{s}$ and depth $>1 \mathrm{ft}$ & $\begin{array}{l}\text { Multivertical grab, }<1.5 \mathrm{ft} / \mathrm{s} \\
\text { and width }>10 \mathrm{ft}(3 \mathrm{~m})\end{array}$ & $\begin{array}{l}\text { Grab in centroid of flow, }<1.5 \mathrm{ft} / \mathrm{s} \text {, } \\
\text { width }<10 \mathrm{ft}(3 \mathrm{~m}) \text { and depth }<1 \mathrm{ft}\end{array}$ \\
\hline \multirow[t]{3}{*}{14} & $\begin{array}{l}\text { Filter with syringe filter from churn or } \\
\text { BGC. }\end{array}$ & $\begin{array}{l}\text { Filter with syringe filter from churn or } \\
\text { BGC. }\end{array}$ & $\begin{array}{l}\text { Filter with syringe filter from churn or } \\
\text { BGC. }\end{array}$ \\
\hline & \multicolumn{3}{|c|}{$\begin{array}{l}\text { Syringe filtration: Prep syringe and filter by (1) pull } 10 \mathrm{~mL} \text { through the needle, invert, plunge to } 30 \mathrm{~mL} \text {, shake and dispose of water, } \\
\text { (2) pull } 5 \mathrm{~mL} \text { through the needle, removing the needle and adding filter, and squirting the water through filter.. }\end{array}$} \\
\hline & $\begin{array}{l}\text { Add } 10 \mathrm{~mL} \text { to } 20 \mathrm{~mL} \text { PEST. } \\
\text { Fill } 40 \mathrm{~mL} \text { half full TX immunoassay } \\
\text { (bottle label IMMGLY). } \\
\text { Add } 10 \mathrm{~mL} \text { to } 20-\mathrm{mL} \text { vial (bottle label } \\
\text { PHARM). } \\
\text { Fill two } 40 \text {-mL vials } 24 \mathrm{~mL} \text { each, KS } \\
\text { Glyphosate (bottle label GLY2). }\end{array}$ & $\begin{array}{l}\text { Add } 10 \mathrm{~mL} \text { to } 20 \mathrm{~mL} \text { PEST. } \\
\text { Fill } 40 \mathrm{~mL} \text { half full TX immunoassay } \\
\text { (bottle label IMMGLY). } \\
\text { Add } 10 \mathrm{~mL} \text { to } 20 \text {-mL vial (bottle label } \\
\text { PHARM). } \\
\text { Fill two } 40 \text {-mL vials } 24 \mathrm{~mL} \text { each, KS } \\
\text { Glyphosate (bottle label GLY2). }\end{array}$ & $\begin{array}{l}\text { Add } 10 \mathrm{~mL} \text { to } 20 \mathrm{~mL} \text { PEST. } \\
\text { Fill } 40 \mathrm{~mL} \text { half full TX immunoassay } \\
\text { (bottle label IMMGLY). } \\
\text { Add } 10 \mathrm{~mL} \text { to } 20 \text {-mL vial (bottle label } \\
\text { PHARM). } \\
\text { Fill two } 40 \text {-mL vials } 24 \mathrm{~mL} \text { each, KS } \\
\text { Glyphosate (bottle label GLY2). }\end{array}$ \\
\hline 15 & $\begin{array}{l}\text { Filter DOC with Aquaprep capsule filter. } \\
\text { Condition aquaprep with organic blank } \\
\text { water. } \\
\text { Let the first } 10 \mathrm{~mL} \text { to waste then fill } \\
\text { two } 40-\mathrm{mL} \text { vials to neck. }\end{array}$ & $\begin{array}{l}\text { Filter DOC with Aquaprep capsule filter. } \\
\text { Condition aquaprep with organic blank } \\
\text { water. } \\
\text { Let the first } 10 \mathrm{~mL} \text { to waste then fill } \\
\text { two } 40-\mathrm{mL} \text { vials to neck. }\end{array}$ & $\begin{array}{l}\text { Filter DOC with Aquaprep capsule filter. } \\
\text { Condition aquaprep with organic blank } \\
\text { water. } \\
\text { Let the first } 10 \mathrm{~mL} \text { to waste then fill } \\
\text { two } 40-\mathrm{mL} \text { vials to neck. }\end{array}$ \\
\hline 16 & $\begin{array}{l}\text { Acidify mercury sample }(10 \mathrm{~mL} \text { of } \\
\text { ultraclean } \mathrm{HCl}) \text {. }\end{array}$ & $\begin{array}{l}\text { Acidify mercury sample }(10 \mathrm{~mL} \text { of } \\
\text { ultraclean } \mathrm{HCl}) \text {. }\end{array}$ & $\begin{array}{l}\text { Acidify mercury sample }(10 \mathrm{~mL} \text { of } \\
\text { ultraclean } \mathrm{HCl}) \text {. }\end{array}$ \\
\hline \multicolumn{4}{|c|}{ Other data collection and clean up } \\
\hline 17 & $\begin{array}{l}\text { Take a reference point measurement for } \\
\text { stage, double-check field sheets for } \\
\text { completeness. }\end{array}$ & $\begin{array}{l}\text { Take a reference point measurement for } \\
\text { stage, double-check field sheets for } \\
\text { completeness. }\end{array}$ & $\begin{array}{l}\text { Take a reference point measurement for } \\
\text { stage, double-check field sheets for } \\
\text { completeness. }\end{array}$ \\
\hline 18 & $\begin{array}{l}\text { Field clean tubing, churn, and 1-L } \\
\text { sample bottle and cap, if re-using. }\end{array}$ & $\begin{array}{l}\text { Field clean tubing, churn, and 1-L } \\
\text { sample bottle and cap, if re-using. }\end{array}$ & $\begin{array}{l}\text { Field clean tubing, churn, and 1-L } \\
\text { sample bottle and cap, if re-using. }\end{array}$ \\
\hline 19 & Field clean tubing for DOC, if re-using. & Field clean tubing for DOC, if re-using. & Field clean tubing for DOC, if re-using. \\
\hline
\end{tabular}

${ }^{1}$ Acid preservation required. 
Table 1.4. Description of the bottle types, laboratory schedules, and preservation protocols by parameter group for samples collected by the U.S. Geological Survey California Stream Quality Assessment study in 2017.

[Laboratories: CA OCRL, U.S. Geological Survey (USGS) California Organic Chemistry Research Laboratory, Sacramento, Calif.; CERC, USGS Columbia Environmental Research Center, Columbia, Mo.; CSL, USGS California Water Science Center Sediment Laboratory, Santa Cruz, Calif.; GD Lab, USGS Geologic Division Laboratory, Denver, Colo.; MRL, USGS Mercury Research Laboratory, Middleton, Wisc.; NWQL, USGS National Water Quality Laboratory, Denver, Colo.; OGRL, USGS Kansas Organic Geochemistry Research Laboratory, Lawrence, Kans.; RSIL, USGS Reston Stable Isotope Laboratory, Reston, Va.; TX WSC, USGS Texas Water Science Center, Austin, Tex. Abbreviations: BEDSED, halogenated compounds, organic wastewater indicators, hormones, PAHs, pesticides, major and trace elements; GLY2, glyphosate by liquid chromatography tandem mass spectrometry; L, liter; MERC, total mercury and total methylmercury; mL, milliliter; N, normal; PAHs, current use pesticides, major and trace elements, radionuclides; POCIS, polar organic chemical integrative sampler; poly, polyetheylene; RTH, richest targeted habitat; >, greater than. Container label assigned by the National Water Quality Laboratory: FA, filtered, acidified cations; FCC, filtered, chilled nutrients; FU, filtered, unpreserved anions; OWI, organic wastewater indicators; PEST, pesticide; PHARM, pharmaceutical; RU, raw, unpreserved physical properties; WCA, whole, chilled, acidified nutrients]

\begin{tabular}{|c|c|c|c|c|}
\hline Parameter group & $\begin{array}{l}\text { Laboratory or } \\
\text { schedule }\end{array}$ & Container (label) & Source & Preservation \\
\hline \multicolumn{5}{|c|}{ Water samples } \\
\hline \multicolumn{5}{|c|}{ BASIC } \\
\hline \multirow[t]{3}{*}{ Major ions } & NWQL 2590 & $\begin{array}{l}\text { 250-mL acid-rinsed poly bottle } \\
\text { (FA) }\end{array}$ & Churn, capsule filter & $\begin{array}{l}7.5 \mathrm{~N} \text { nitric } \\
\text { acid }\end{array}$ \\
\hline & & 250-mL clear poly bottle (FU) & Churn, capsule filter & \\
\hline & & 250-mL clear poly bottle (RU) & Churn, raw & \\
\hline \multirow[t]{2}{*}{ Nutrients } & NWQL 2711 & $\begin{array}{l}\text { 125-mL white poly bottle } \\
\text { (WCA) }\end{array}$ & Churn, raw & $\begin{array}{l}4.5 \mathrm{~N} \text { sulfuric } \\
\text { acid }\end{array}$ \\
\hline & & $\begin{array}{l}125-\mathrm{mL} \text { brown poly bottle } \\
\text { (FCC) }\end{array}$ & Churn, capsule filter & \\
\hline $\begin{array}{l}\text { Dissolved organic carbon and } \\
\text { ultraviolet absorbance at } 254 \\
\text { nanometers }\end{array}$ & MRL & $\begin{array}{l}\text { (2) } 40-\mathrm{mL} \text { amber glass septum } \\
\text { vials }\end{array}$ & $\begin{array}{l}\text { Grab, } 250-\mathrm{mL} \\
\text { amber glass, disc } \\
\text { filter }\end{array}$ & \\
\hline $\begin{array}{l}\text { Suspended sediment (concentration } \\
\text { only) }\end{array}$ & CSL & 1-L poly wide-mouth bottle & Churn, raw & \\
\hline Immunoassay (glyphosate) & TX WSC & 40-mL amber glass septum vial & Churn, syringe filter & $\begin{array}{l}\text { Freeze if held } \\
>\text { week }\end{array}$ \\
\hline \multicolumn{5}{|c|}{ OWI } \\
\hline Organic wastewater indicators (OWI) & NWQL 4433 & $\begin{array}{l}\text { 1-L, baked amber glass bottle } \\
\text { (OWI) }\end{array}$ & Churn, raw & \\
\hline \multicolumn{5}{|c|}{ MERC } \\
\hline $\begin{array}{l}\text { Total and methylmercury, filtered water } \\
\text { and particulate }\end{array}$ & MRL & 1-L Nalgene bottle & Grab & $\begin{array}{l}\text { Ultrapure } \\
\text { hydrochloric } \\
\text { acid }\end{array}$ \\
\hline \multicolumn{5}{|c|}{ ISOTOPES } \\
\hline Nitrogen and oxygen isotopes & RSIL & 125 -mL clear poly bottle & Churn, capsule filter & \\
\hline \multicolumn{5}{|c|}{ Sediment samples } \\
\hline
\end{tabular}


Table 1.4. Description of the bottle types, laboratory schedules, and preservation protocols by parameter group for samples collected by the U.S. Geological Survey California Stream Quality Assessment study in 2017.—Continued

[Laboratories: CA OCRL, U.S. Geological Survey (USGS) California Organic Chemistry Research Laboratory, Sacramento, Calif.; CERC, USGS Columbia Environmental Research Center, Columbia, Mo.; CSL, USGS California Water Science Center Sediment Laboratory, Santa Cruz, Calif.; GD Lab, USGS Geologic Division Laboratory, Denver, Colo.; MRL, USGS Mercury Research Laboratory, Middleton, Wisc.; NWQL, USGS National Water Quality Laboratory, Denver, Colo.; OGRL, USGS Kansas Organic Geochemistry Research Laboratory, Lawrence, Kans.; RSIL, USGS Reston Stable Isotope Laboratory, Reston, Va.; TX WSC, USGS Texas Water Science Center, Austin, Tex. Abbreviations: BEDSED, halogenated compounds, organic wastewater indicators, hormones, PAHs, pesticides, major and trace elements; GLY2, glyphosate by liquid chromatography tandem mass spectrometry; L, liter; MERC, total mercury and total methylmercury; mL, milliliter; N, normal; PAHs, current use pesticides, major and trace elements, radionuclides; POCIS, polar organic chemical integrative sampler; poly, polyetheylene; RTH, richest targeted habitat; >, greater than. Container label assigned by the National Water Quality Laboratory: FA, filtered, acidified cations; FCC, filtered, chilled nutrients; FU, filtered, unpreserved anions; OWI, organic wastewater indicators; PEST, pesticide; PHARM, pharmaceutical; RU, raw, unpreserved physical properties; WCA, whole, chilled, acidified nutrients]

\begin{tabular}{|c|c|c|c|c|}
\hline Parameter group & $\begin{array}{l}\text { Laboratory or } \\
\text { schedule }\end{array}$ & Container (label) & Source & Preservation \\
\hline \multicolumn{5}{|c|}{ BEDSED } \\
\hline $\begin{array}{l}\text { Polycyclic aromatic hydrocarbons and } \\
\text { other semivolatile organic compounds }\end{array}$ & NWQL 5506 & $\begin{array}{l}\text { 125-mL baked amber glass } \\
\text { wide-mouth jar }\end{array}$ & Composite sediment & \\
\hline Current use pesticides & CA OCRL & $\begin{array}{l}\text { 125-mL baked amber glass } \\
\text { wide-mouth jar }\end{array}$ & Composite sediment & \\
\hline Major and trace elements & GD Lab & $125-\mathrm{mL}$ poly wide-mouth jar & Composite sediment & \\
\hline Hormones & NWQL 4434 & $\begin{array}{l}\text { 125-mL baked amber glass } \\
\text { wide-mouth jar }\end{array}$ & Composite sediment & \\
\hline \multicolumn{5}{|c|}{ Algae samples } \\
\hline \multicolumn{5}{|c|}{$\mathrm{CHL}$} \\
\hline Periphyton biomass, chlorophyll-a & NWQL 1632 & (2) glass-fiber filters (GFF) & RTH, GFF filter & Freeze \\
\hline \multicolumn{5}{|c|}{ POCIS samplers } \\
\hline \multicolumn{5}{|c|}{ POCIS } \\
\hline Current use pesticides & CERC & & POCIS & \\
\hline Organic wastewater indicators (OWI) & CERC & & POCIS & \\
\hline Pharmaceuticals & CERC & & POCIS & \\
\hline
\end{tabular}


Table 1.5. Deployment dates for Polar Organic Chemical Integrative Samplers deployed by the U.S. Geological Survey California Stream Quality Assessment in 2017.

[Begin date, date that POCIS (polar organic chemical integrative samplers) were deployed in the stream; End date, date that POCIS were retrieved from the stream; g, gram; mm/dd/yyyy, month/day/year; N/A, not applicable; No., number; NWIS, U.S. Geological Survey National Water Information System database; wt, weight]

\begin{tabular}{|c|c|c|c|c|c|c|c|}
\hline NWIS station number & Field identifier & $\begin{array}{l}\text { No. of } \\
\text { POCIS }\end{array}$ & $\begin{array}{l}\text { Sorbent wt } \\
\text { per POCIS } \\
\text { (g) }\end{array}$ & $\begin{array}{l}\text { Begin date } \\
\text { (mm/dd/yyyy) }\end{array}$ & $\begin{array}{l}\text { End date } \\
\text { (mm/dd/yyyy) }\end{array}$ & $\begin{array}{l}\text { No. } \\
\text { days }\end{array}$ & Sample type \\
\hline 383719122462501 & CA_Maacama & 2 & 0.2 & $04 / 03 / 2017$ & 05/08/2017 & 35 & Environmental \\
\hline 11465350 & CA_DryMouth & 2 & 0.2 & $04 / 03 / 2017$ & 05/08/2017 & 35 & Environmental \\
\hline 11465350 & CA_DryMouth & 2 & 0.2 & $04 / 03 / 2017$ & 05/08/2017 & 35 & Replicate \\
\hline 11465350 & CA_DryMouth & 2 & 0.2 & $04 / 03 / 2017$ & 05/08/2017 & 35 & Field blank \\
\hline 11466800 & CA_MarkWMir & 2 & 0.2 & $04 / 04 / 2017$ & 05/09/2017 & 35 & Environmental \\
\hline 11466320 & CA_SantaWillow & 2 & 0.2 & $04 / 05 / 2017$ & 05/10/2017 & 35 & Environmental \\
\hline 382619122531401 & CA_Green & 2 & 0.2 & $04 / 05 / 2017$ & 05/10/2017 & 35 & Environmental \\
\hline 11466170 & CA_Matanzas & 2 & 0.2 & $04 / 04 / 2017$ & 05/09/2017 & 35 & Environmental \\
\hline 11466200 & CA_SantaSanta & 2 & 0.2 & $04 / 06 / 2017$ & $05 / 11 / 2017$ & 35 & Environmental \\
\hline 11465690 & CA_Colgan & 2 & 0.2 & $04 / 06 / 2017$ & 05/11/2017 & 35 & Environmental \\
\hline 382346122521201 & CA_NoNameMonty & 2 & 0.2 & $04 / 05 / 2017$ & 05/10/2017 & 35 & Environmental \\
\hline 11465660 & CA_Copeland & 2 & 0.2 & 04/04/2017 & 05/09/2017 & 35 & Environmental \\
\hline 11458500 & CA_SonomaAgua & 2 & 0.2 & $04 / 03 / 2017$ & 05/08/2017 & 35 & Environmental \\
\hline 381740122395901 & CA_Lichau & 2 & 0.2 & $04 / 04 / 2017$ & 05/09/2017 & 35 & Environmental \\
\hline 381556122280201 & CA_SonomaWat & 2 & 0.2 & $04 / 03 / 2017$ & 05/08/2017 & 35 & Environmental \\
\hline 381519122385601 & CA_Petaluma & 2 & 0.2 & $04 / 04 / 2017$ & 05/09/2017 & 35 & Environmental \\
\hline 11459500 & CA_Novato & 2 & 0.2 & $04 / 05 / 2017$ & 05/10/2017 & 35 & Environmental \\
\hline 11459500 & CA_Novato & 2 & 0.2 & $04 / 05 / 2017$ & 05/10/2017 & 35 & Replicate \\
\hline 11459500 & CA_Novato & 2 & 0.2 & $04 / 05 / 2017$ & 05/10/2017 & 35 & Field blank \\
\hline 380410122315501 & CA_ArroyoDig & 2 & 0.2 & $04 / 05 / 2017$ & 05/10/2017 & 35 & Environmental \\
\hline 380345122345201 & CA_ArroyoNov & 2 & 0.2 & $04 / 03 / 2017$ & 05/08/2017 & 35 & Environmental \\
\hline 11460000 & CA_Corte & 2 & 0.2 & $04 / 06 / 2017$ & 05/11/2017 & 35 & Environmental \\
\hline 383321122302101 & CA_NapaBale & 2 & 0.2 & $04 / 04 / 2017$ & 05/09/2017 & 35 & Environmental \\
\hline 383305122311901 & CA_Ritchey & 2 & 0.2 & $04 / 03 / 2017$ & 05/08/2017 & 35 & Environmental \\
\hline 383109122363301 & CA_MarkWTar & 2 & 0.2 & $04 / 03 / 2017$ & 05/08/2017 & 35 & Environmental \\
\hline 382634122315201 & CA_SonomaAdobe & 2 & 0.2 & $04 / 03 / 2017$ & 05/08/2017 & 35 & Environmental \\
\hline 382245122001601 & CA_Ulatis & 2 & 0.2 & $04 / 03 / 2017$ & 05/08/2017 & 35 & Environmental \\
\hline 11456500 & CA_ConnOak & 2 & 0.2 & $04 / 04 / 2017$ & 05/09/2017 & 35 & Environmental \\
\hline 382017122161101 & CA_Milliken & 2 & 0.2 & 04/06/2017 & 05/11/2017 & 35 & Environmental \\
\hline 11458300 & CA_NapaNapa & 2 & 0.2 & $04 / 05 / 2017$ & 05/10/2017 & 35 & Environmental \\
\hline 11458300 & CA_NapaNapa & 2 & 0.2 & $04 / 05 / 2017$ & 05/10/2017 & 35 & Replicate \\
\hline 11458300 & CA_NapaNapa & 2 & 0.2 & $04 / 05 / 2017$ & 05/10/2017 & 35 & Field blank \\
\hline 381441122064301 & CA_Suisan & 2 & 0.2 & $04 / 06 / 2017$ & 05/11/2017 & 35 & Environmental \\
\hline 11182400 & CA_ArroyoMart & 2 & 0.2 & $04 / 05 / 2017$ & 05/10/2017 & 35 & Environmental \\
\hline 375819122035801 & CA_Grayson & 2 & 0.2 & $04 / 06 / 2017$ & 05/11/2017 & 35 & Environmental \\
\hline 375819122035801 & CA_Grayson & 2 & 0.2 & $04 / 06 / 2017$ & 05/11/2017 & 35 & Replicate \\
\hline 375819122035801 & CA_Grayson & 2 & 0.2 & $04 / 06 / 2017$ & 05/11/2017 & 35 & Field blank \\
\hline 375808122172601 & CA_Wilkie & 2 & 0.2 & $04 / 03 / 2017$ & 05/08/2017 & 35 & Environmental \\
\hline 375746122195501 & CA_SanPabloPort & 2 & 0.2 & $04 / 03 / 2017$ & 05/08/2017 & 35 & Environmental \\
\hline 375701121564401 & CA_MtDiablo & 2 & 0.2 & 04/06/2017 & 05/11/2017 & 35 & Environmental \\
\hline
\end{tabular}


Table 1.5. Deployment dates for Polar Organic Chemical Integrative Samplers deployed by the U.S. Geological Survey California Stream Quality Assessment in 2017.-Continued

[Begin date, date that POCIS (polar organic chemical integrative samplers) were deployed in the stream; End date, date that POCIS were retrieved from the stream; g, gram; mm/dd/yyyy, month/day/year; N/A, not applicable; No., number; NWIS, U.S. Geological Survey National Water Information System database; wt, weight]

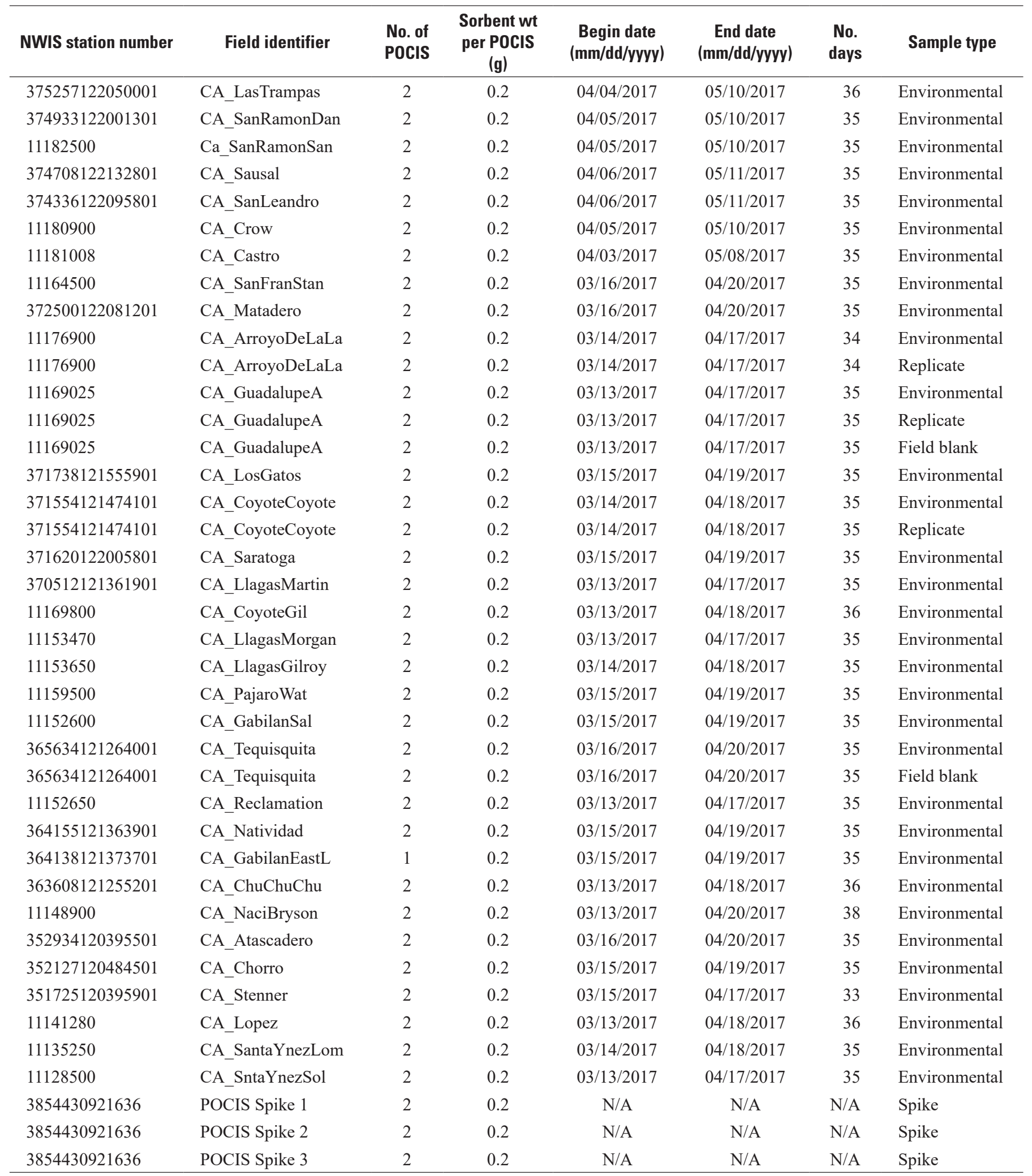


Table 1.6. Deployment dates for ceramic tiles used to collect biofilms for pesticide analysis as part of the U.S. Geological Survey California Stream Quality Assessment study in 2017.

[Begin date, date that tiles were deployed in the stream; End date, date that tiles were retrieved from the stream; mm/dd/yyyy, month/day/year; No., number; NWIS, U.S. Geological Survey National Water Information System database]

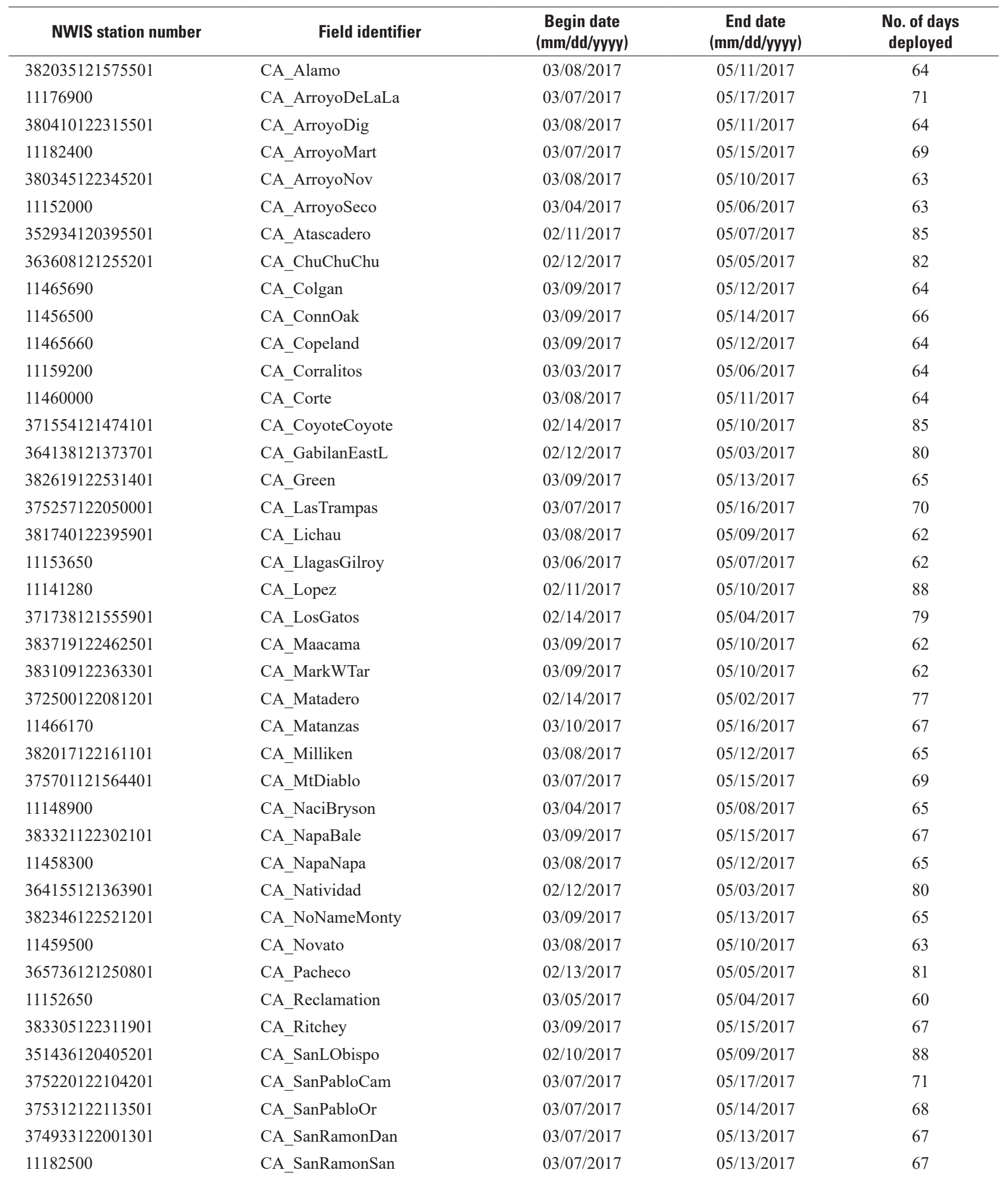


Table 1.6. Deployment dates for ceramic tiles used to collect biofilms for pesticide analysis as part of the U.S. Geological Survey California Stream Quality Assessment study in 2017.-Continued

[Begin date, date that tiles were deployed in the stream; End date, date that tiles were retrieved from the stream; mm/dd/yyyy, month/day/year; No., number; NWIS, U.S. Geological Survey National Water Information System database]

\begin{tabular}{lllc}
\hline \multicolumn{1}{c}{ NWIS station number } & \multicolumn{1}{c}{ Field identifier } & $\begin{array}{c}\text { Begin date } \\
\text { (mm/dd/yvy) }\end{array}$ & $\begin{array}{c}\text { End date } \\
\text { (mm/dd/yvyv) }\end{array}$ \\
\hline 11466200 & CA_SantaSanta & $03 / 10 / 2017$ & $05 / 16 / 2017$ \\
11466320 & CA_SantaWillow & $03 / 10 / 2017$ & $05 / 11 / 2017$ \\
11135250 & CA_SantaYnezLom & $02 / 10 / 2017$ & $05 / 11 / 2017$ \\
371620122005801 & CA_Saratoga & $02 / 14 / 2017$ & $05 / 03 / 2017$ \\
374708122132801 & CA_Sausal & $03 / 08 / 2017$ & $05 / 12 / 2017$ \\
382634122315201 & CA_SonomaAdobe & $03 / 09 / 2017$ & $05 / 17 / 2017$ \\
381441122064301 & CA_Suisan & $03 / 08 / 2017$ & $05 / 12 / 2017$ \\
365634121264001 & CA_Tequisquita & $02 / 13 / 2017$ & $05 / 05 / 2017$ \\
382245122001601 & CA_Ulatis & $03 / 08 / 2017$ & $05 / 11 / 2017$ \\
365955121350601 & CA_UvasMiller & $02 / 13 / 2017$ & $05 / 07 / 2017$ \\
375413122033301 & CA_Walnut & $03 / 07 / 2017$ & 65 \\
375808122172601 & CA_Wilkie & $03 / 07 / 2017$ & $65 / 16 / 2017$ \\
383039122502401 & CA_Windsor & $03 / 10 / 2017$ & $05 / 13 / 2017$ \\
\hline
\end{tabular}




\section{Appendix 2 Description of the U.S. Geological Survey National Water Quality Laboratory Schedules Used for Water, Bed Sediment, and Biofilms}

Table 2.1. U.S. Geological Survey National Water Quality Laboratory Schedule 2590 for major ions in water.

[CAS, Chemical Abstracts Service1; IC, ion chromatography; ICP/AES, inductively coupled plasma/atomic emission spectroscopy; ltmdl, long-term method detection limit; mg/L, milligram per liter; mrl, minimum reporting level; NWIS, USGS National Water Information System; NWQL, U.S. Geological Survey (USGS) National Water Quality Laboratory; - , no data; $\mu \mathrm{S} / \mathrm{cm}$, microsiemens per centimeter at 25 degrees Celsius]

\begin{tabular}{|c|c|c|c|c|c|c|c|c|c|}
\hline Parameter name & $\begin{array}{c}\text { NWOL } \\
\text { lab } \\
\text { code }\end{array}$ & $\begin{array}{c}\text { NWIS } \\
\text { parameter } \\
\text { code }\end{array}$ & $\begin{array}{l}\text { NWIS } \\
\text { method } \\
\text { code }\end{array}$ & $\begin{array}{c}\text { CAS } \\
\text { number }\end{array}$ & $\begin{array}{l}\text { Reporting } \\
\text { level }\end{array}$ & Unit & $\begin{array}{l}\text { Reporting } \\
\text { level type }\end{array}$ & Method citation & Method \\
\hline Calcium & 659 & 00915 & PLA11 & $7440-70-2$ & 0.022 & $\mathrm{mg} / \mathrm{L}$ & ltmdl & Fishman (1993) & ICP/AES \\
\hline Chloride & 1571 & 00940 & IC022 & $16887-00-6$ & 0.02 & $\mathrm{mg} / \mathrm{L}$ & ltmdl & $\begin{array}{l}\text { Fishman and } \\
\text { Friedman (1989) }\end{array}$ & IC \\
\hline Magnesium & 663 & 00925 & PLA11 & $7439-95-4$ & 0.011 & $\mathrm{mg} / \mathrm{L}$ & ltmdl & Fishman (1993) & ICP/AES \\
\hline Potassium & 2773 & 00935 & PLO03 & 7440-09-7 & 0.03 & $\mathrm{mg} / \mathrm{L}$ & ltmdl & Fishman (1993) & ICP/AES \\
\hline Sodium & 675 & 00930 & PLA11 & $7440-23-5$ & 0.06 & $\mathrm{mg} / \mathrm{L}$ & ltmdl & Fishman (1993) & ICP/AES \\
\hline $\begin{array}{l}\text { Specific conductance, } \\
\text { laboratory }\end{array}$ & 69 & 90095 & WHT03 & - & 5 & $\mu \mathrm{S} / \mathrm{cm}$ & $\mathrm{mrl}$ & $\begin{array}{l}\text { Fishman and } \\
\text { Friedman (1989) }\end{array}$ & $\begin{array}{l}\text { Wheatstone } \\
\text { Bridge }\end{array}$ \\
\hline Sulfate & 1572 & 00945 & IC022 & $14808-79-8$ & 0.02 & $\mathrm{mg} / \mathrm{L}$ & ltmdl & $\begin{array}{l}\text { Fishman and } \\
\text { Friedman (1989) }\end{array}$ & IC \\
\hline
\end{tabular}

${ }^{1}$ This report contains CAS Registry Numbers ${ }^{\circledR}$, which is a Registered Trademark of the American Chemical Society. CAS recommends the verification of the CAS Registry Numbers through CAS Client Services $\mathrm{SM}$.

Table 2.2. U.S. Geological Survey National Water Quality Laboratory Schedule 2711 for nutrients in water.

[CAS, Chemical Abstracts Service1; EPA, U.S. Environmental Protection Agency; ltmdl, long-term method detection limit; mdl, method detection limit; mg/L, milligram per liter; N, nitrogen; NWIS, USGS National Water Information System; NWQL, U.S. Geological Survey (USGS) National Water Quality Laboratory; - , no data]

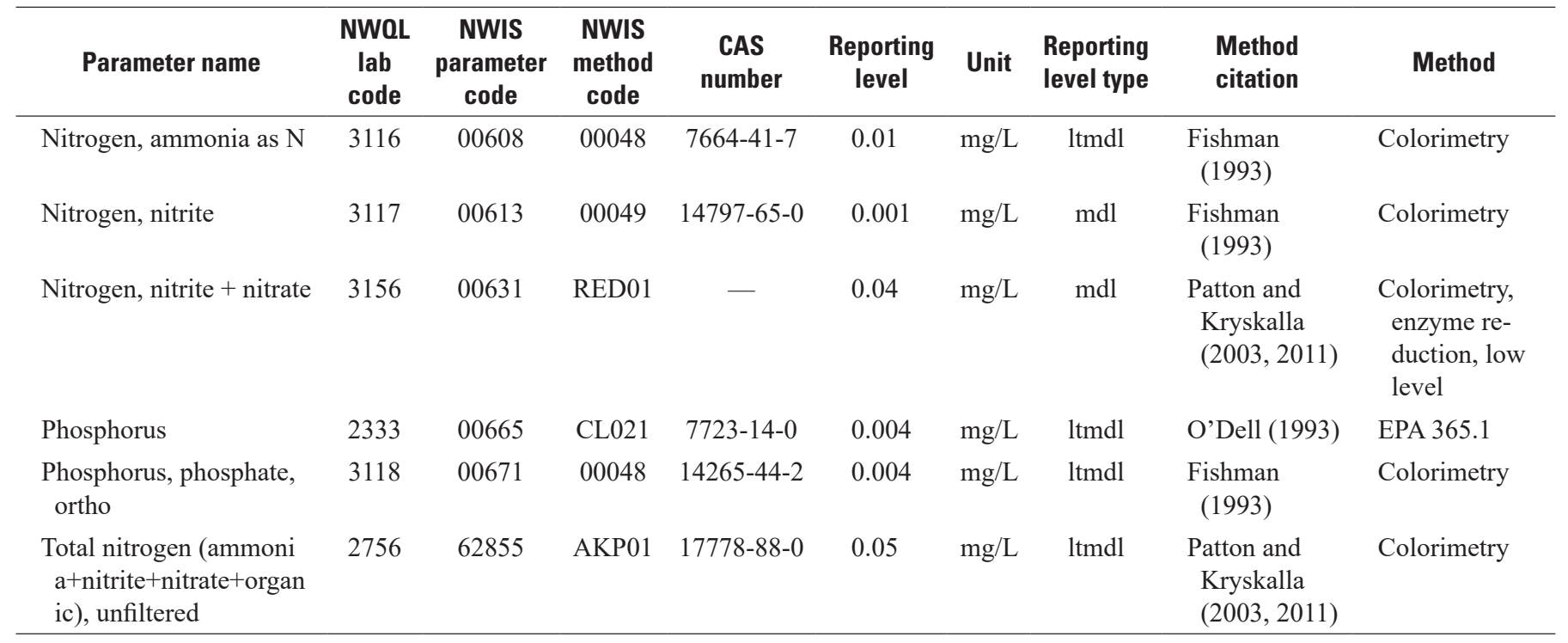

${ }^{1}$ This report contains CAS Registry Numbers ${ }^{\circledR}$, which is a Registered Trademark of the American Chemical Society. CAS recommends the verification of the CAS Registry Numbers through CAS Client ServicesSM. 
Table 2.3.1. U.S. Geological National Water Quality Laboratory Schedule 2437 for current-use pesticides in filtered water.

[Methods are described in Sandstrom and others (2016). Parameter names are as shown in the U.S. Geological Survey (USGS) National Water Information System (NWIS) database and do not include special characters or formatting. Abbreviations: CAS, Chemical Abstracts Service1; N/A, not applicable; ng/L, nanogram per liter; NWQL, USGS National Water Quality Laboratory; pct, percent; RLDQC, reporting level based on DQCALC software and procedure, as described in NWQL Technical Memorandum 15.02; - , no data]

\begin{tabular}{|c|c|c|c|c|c|c|}
\hline Parameter name & $\begin{array}{c}\text { NWIS } \\
\text { parameter } \\
\text { code }\end{array}$ & $\begin{array}{l}\text { NWIS } \\
\text { method } \\
\text { code }\end{array}$ & CAS number & $\begin{array}{l}\text { Reporting } \\
\text { level }\end{array}$ & Unit & $\begin{array}{l}\text { Reporting } \\
\text { level type }\end{array}$ \\
\hline $1 \mathrm{H}-1,2,4$-Triazole & 68498 & LCM60 & $288-88-0$ & 22 & $\mathrm{ng} / \mathrm{L}$ & RLDQC \\
\hline 2,3,3-Trichloro-2-propene-1-sulfonic acid (TCPSA) & 68691 & LCM60 & $65600-62-6$ & 55 & $\mathrm{ng} / \mathrm{L}$ & RLDQC \\
\hline 2,4-D & 68500 & LCM60 & $94-75-7$ & 62 & $\mathrm{ng} / \mathrm{L}$ & RLDQC \\
\hline 2,4-D-d3, surrogate, water,filtered, percent recovery (surrogate) & 91986 & LCM60 & $202480-67-9$ & $\mathrm{~N} / \mathrm{A}$ & pct & $\mathrm{N} / \mathrm{A}$ \\
\hline 2-(1-Hydroxyethyl)-6-methylaniline & 68611 & LCM60 & $196611-19-5$ & 54 & $\mathrm{ng} / \mathrm{L}$ & RLDQC \\
\hline 2-[(2-Ethyl-6-methylphenyl)amino]-1-propanol & 68595 & LCM60 & $61520-53-4$ & 5 & $\mathrm{ng} / \mathrm{L}$ & RLDQC \\
\hline 2-Amino-N-isopropylbenzamide & 68503 & LCM60 & $30391-89-0$ & 4 & $\mathrm{ng} / \mathrm{L}$ & RLDQC \\
\hline 2-Aminobenzimidazole & 68502 & LCM60 & $934-32-7$ & 9 & $\mathrm{ng} / \mathrm{L}$ & RLDQC \\
\hline 2-Chloro-2',6'-diethylacetanilide & 68525 & LCM60 & $6967-29-9$ & 5 & $\mathrm{ng} / \mathrm{L}$ & RLDQC \\
\hline 2-Chloro-4,6-diamino-s-triazine $\{\mathrm{CAAT}\}$ (Didealkylatrazine) & 68547 & LCM60 & $3397-62-4$ & 24 & $\mathrm{ng} / \mathrm{L}$ & RLDQC \\
\hline 2-Chloro-4-isopropylamino-6-amino-s-triazine & 68552 & LCM60 & $6190-65-4$ & 11 & $\mathrm{ng} / \mathrm{L}$ & RLDQC \\
\hline 2-Chloro-6-ethylamino-4-amino-s-triazine $\{\mathrm{CEAT}\}$ & 68550 & LCM60 & $1007-28-9$ & 20 & $\mathrm{ng} / \mathrm{L}$ & RLDQC \\
\hline 2-Chloro-N-(2-ethyl-6-methylphenyl)acetamide & 68521 & LCM60 & $32428-71-0$ & 5 & $\mathrm{ng} / \mathrm{L}$ & RLDQC \\
\hline 2-Hydroxy-4-isopropylamino-6-amino-s-triazine & 68659 & LCM60 & 19988-24-0 & 4 & $\mathrm{ng} / \mathrm{L}$ & RLDQC \\
\hline 2-Hydroxy-4-isopropylamino-6-ethylamino-s-triazine $\{\mathrm{OIET}\}$ & 68660 & LCM60 & $2163-68-0$ & 8 & $\mathrm{ng} / \mathrm{L}$ & RLDQC \\
\hline 2-Hydroxy-6-ethylamino-4-amino-s-triazine & 68656 & LCM60 & $7313-54-4$ & 100 & $\mathrm{ng} / \mathrm{L}$ & RLDQC \\
\hline 2-Isopropyl-6-methyl-4-pyrimidinol & 68505 & LCM60 & $2814-20-2$ & 8 & $\mathrm{ng} / \mathrm{L}$ & RLDQC \\
\hline 3,4-Dichlorophenylurea & 68226 & LCM60 & $2327-02-8$ & 108 & $\mathrm{ng} / \mathrm{L}$ & RLDQC \\
\hline 3-Hydroxycarbofuran & 68508 & LCM60 & $16655-82-6$ & 16 & $\mathrm{ng} / \mathrm{L}$ & RLDQC \\
\hline 3-Phenoxybenzoic acid & 68873 & LCM60 & $3739-38-6$ & 61 & $\mathrm{ng} / \mathrm{L}$ & RLDQC \\
\hline 3-Phenoxybenzoic acid-13C6 (surrogate) & 90516 & LCM60 & - & N/A & pct & N/A \\
\hline 4-(Hydroxymethyl)pendimethalin & 68511 & LCM60 & $56750-76-6$ & 114 & $\mathrm{ng} / \mathrm{L}$ & RLDQC \\
\hline 4-Chlorobenzylmethyl sulfoxide & 68514 & LCM60 & $24176-68-9$ & 3.2 & $\mathrm{ng} / \mathrm{L}$ & RLDQC \\
\hline 4-Hydroxy molinate & 68515 & LCM60 & $66747-12-4$ & 7 & $\mathrm{ng} / \mathrm{L}$ & RLDQC \\
\hline 4-Hydroxychlorothalonil & 68336 & LCM60 & $28343-61-5$ & 98 & $\mathrm{ng} / \mathrm{L}$ & RLDQC \\
\hline 4-Hydroxyhexazinone A & 68517 & LCM60 & $72576-13-7$ & 3 & $\mathrm{ng} / \mathrm{L}$ & RLDQC \\
\hline Acephate & 68519 & LCM60 & $30560-19-1$ & 10 & $\mathrm{ng} / \mathrm{L}$ & RLDQC \\
\hline Acetochlor & 68520 & LCM60 & $34256-82-1$ & 10 & $\mathrm{ng} / \mathrm{L}$ & RLDQC \\
\hline Acetochlor oxanilic acid & 68522 & LCM60 & $194992-44-4$ & 65 & $\mathrm{ng} / \mathrm{L}$ & RLDQC \\
\hline Acetochlor sulfonic acid & 68523 & LCM60 & $187022-11-3$ & 320 & $\mathrm{ng} / \mathrm{L}$ & RLDQC \\
\hline Acetochlor sulfynilacetic acid & 68524 & LCM60 & $618113-86-3$ & 176 & $\mathrm{ng} / \mathrm{L}$ & RLDQC \\
\hline Acetochlor-d11 (surrogate) & 90517 & LCM60 & $1189897-44-6$ & $\mathrm{~N} / \mathrm{A}$ & pct & $\mathrm{N} / \mathrm{A}$ \\
\hline Alachlor & 65064 & LCM60 & $15972-60-8$ & 27 & $\mathrm{ng} / \mathrm{L}$ & RLDQC \\
\hline Alachlor oxanilic acid & 68526 & LCM60 & $171262-17-2$ & 60 & $\mathrm{ng} / \mathrm{L}$ & RLDQC \\
\hline Alachlor sulfonic acid & 68871 & LCM60 & $142363-53-9$ & 360 & $\mathrm{ng} / \mathrm{L}$ & RLDQC \\
\hline Alachlor sulfynilacetic acid & 68527 & LCM60 & $494847-39-1$ & 128 & $\mathrm{ng} / \mathrm{L}$ & RLDQC \\
\hline Alachlor-d13 (surrogate) & 90518 & LCM60 & $1015856-63-9$ & $\mathrm{~N} / \mathrm{A}$ & pct & N/A \\
\hline Aldicarb & 68528 & LCM60 & $116-06-3$ & 8 & $\mathrm{ng} / \mathrm{L}$ & RLDQC \\
\hline Aldicarb sulfone & 68529 & LCM60 & $1646-88-4$ & 20 & $\mathrm{ng} / \mathrm{L}$ & RLDQC \\
\hline
\end{tabular}


Table 2.3.1. U.S. Geological National Water Quality Laboratory Schedule 2437 for current-use pesticides in filtered water-—Continued

[Methods are described in Sandstrom and others (2016). Parameter names are as shown in the U.S. Geological Survey (USGS) National Water Information System (NWIS) database and do not include special characters or formatting. Abbreviations: CAS, Chemical Abstracts Service ${ }^{1}$; N/A, not applicable; ng/L, nanogram per liter; NWQL, USGS National Water Quality Laboratory; pct, percent; RLDQC, reporting level based on DQCALC software and procedure, as described in NWQL Technical Memorandum 15.02; - , no data]

\begin{tabular}{|c|c|c|c|c|c|c|}
\hline Parameter name & $\begin{array}{c}\text { NWIS } \\
\text { parameter } \\
\text { code }\end{array}$ & $\begin{array}{c}\text { NWIS } \\
\text { method } \\
\text { code }\end{array}$ & CAS number & $\begin{array}{l}\text { Reporting } \\
\text { level }\end{array}$ & Unit & $\begin{array}{l}\text { Reporting } \\
\text { level type }\end{array}$ \\
\hline Aldicarb sulfoxide & 68530 & LCM60 & $1646-87-3$ & 2.2 & $\mathrm{ng} / \mathrm{L}$ & RLDQC \\
\hline Ametryn & 68533 & LCM60 & $834-12-8$ & 2.6 & $\mathrm{ng} / \mathrm{L}$ & RLDQC \\
\hline Asulam & 68536 & LCM60 & $3337-71-1$ & 50 & $\mathrm{ng} / \mathrm{L}$ & RLDQC \\
\hline Atrazine & 65065 & LCM60 & $1912-24-9$ & 6.8 & $\mathrm{ng} / \mathrm{L}$ & RLDQC \\
\hline Azinphos-methyl & 65066 & LCM60 & $86-50-0$ & 8 & $\mathrm{ng} / \mathrm{L}$ & RLDQC \\
\hline Azinphos-methyl oxon & 68211 & LCM60 & $961-22-8$ & 15 & $\mathrm{ng} / \mathrm{L}$ & RLDQC \\
\hline Azoxystrobin & 66589 & LCM60 & $131860-33-8$ & 3 & $\mathrm{ng} / \mathrm{L}$ & RLDQC \\
\hline Bentazon & 68538 & LCM60 & $25057-89-0$ & 9 & $\mathrm{ng} / \mathrm{L}$ & RLDQC \\
\hline Bifenthrin & 65067 & LCM60 & $82657-04-3$ & 19 & $\mathrm{ng} / \mathrm{L}$ & RLDQC \\
\hline Bromacil & 68542 & LCM60 & $314-40-9$ & 5.6 & $\mathrm{ng} / \mathrm{L}$ & RLDQC \\
\hline Bromoxynil & 68543 & LCM60 & $1689-84-5$ & 60 & $\mathrm{ng} / \mathrm{L}$ & RLDQC \\
\hline Butachlor sulfonic acid (surrogate) & 90624 & LCM60 & $187022-12-4$ & $\mathrm{~N} / \mathrm{A}$ & pct & N/A \\
\hline Butralin & 68545 & LCM60 & $33629-47-9$ & 5 & $\mathrm{ng} / \mathrm{L}$ & RLDQC \\
\hline Butylate & 65068 & LCM60 & $2008-41-5$ & 10 & $\mathrm{ng} / \mathrm{L}$ & RLDQC \\
\hline Carbaryl & 65069 & LCM60 & $63-25-2$ & 5.6 & $\mathrm{ng} / \mathrm{L}$ & RLDQC \\
\hline Carbaryl-d7 (surrogate) & 90519 & LCM60 & $362049-56-7$ & N/A & pct & N/A \\
\hline Carbendazim & 68548 & LCM60 & $10605-21-7$ & 10 & $\mathrm{ng} / \mathrm{L}$ & RLDQC \\
\hline Carbendazim-d4 (surrogate) & 90520 & LCM60 & $291765-95-2$ & $\mathrm{~N} / \mathrm{A}$ & pct & N/A \\
\hline Carbofuran & 65070 & LCM60 & $1563-66-2$ & 5 & $\mathrm{ng} / \mathrm{L}$ & RLDQC \\
\hline Carbofuran-d3 (surrogate) & 90521 & LCM60 & $1007459-98-4$ & $\mathrm{~N} / \mathrm{A}$ & pct & $\mathrm{N} / \mathrm{A}$ \\
\hline Carboxy molinate & 68549 & LCM60 & $66747-13-5$ & 54 & $\mathrm{ng} / \mathrm{L}$ & RLDQC \\
\hline Chlorimuron-ethyl & 68872 & LCM60 & $90982-32-4$ & 8.8 & $\mathrm{ng} / \mathrm{L}$ & RLDQC \\
\hline Chlorosulfonamide acid & 68551 & LCM60 & - & 60 & $\mathrm{ng} / \mathrm{L}$ & RLDQC \\
\hline Chlorpyrifos & 65072 & LCM60 & $2921-88-2$ & 3 & $\mathrm{ng} / \mathrm{L}$ & RLDQC \\
\hline Chlorpyrifos oxon & 68216 & LCM60 & - & 4.4 & $\mathrm{ng} / \mathrm{L}$ & RLDQC \\
\hline Chlorsulfuron & 61678 & LCM60 & $64902-72-3$ & 50 & $\mathrm{ng} / \mathrm{L}$ & RLDQC \\
\hline cis-Bifenthrin acid/cis-Cyhalothrin acid/cis-Tefluthrin acid & 68553 & LCM60 & $68127-59-3$ & 105 & $\mathrm{ng} / \mathrm{L}$ & RLDQC \\
\hline cis-Permethrin & 68769 & LCM60 & $61949-76-6$ & 4.2 & $\mathrm{ng} / \mathrm{L}$ & RLDQC \\
\hline cis-Permethrin-13C6 (surrogate) & 90558 & LCM60 & - & $\mathrm{N} / \mathrm{A}$ & pct & N/A \\
\hline Cyanazine & 66592 & LCM60 & $21725-46-2$ & 50 & $\mathrm{ng} / \mathrm{L}$ & RLDQC \\
\hline Dacthal monoacid & 68560 & LCM60 & $887-54-7$ & 2700 & $\mathrm{ng} / \mathrm{L}$ & RLDQC \\
\hline Dechlorofipronil & 68561 & LCM60 & - & 3.8 & $\mathrm{ng} / \mathrm{L}$ & RLDQC \\
\hline Dechlorometolachlor & 68562 & LCM60 & $126605-22-9$ & 2 & $\mathrm{ng} / \mathrm{L}$ & RLDQC \\
\hline Deethylatrazine-d6 (surrogate) & 90522 & LCM60 & - & N/A & pct & N/A \\
\hline Deiodo flubendiamide & 68563 & LCM60 & $1016160-78-3$ & 10 & $\mathrm{ng} / \mathrm{L}$ & RLDQC \\
\hline Deisopropyl prometryn & 68564 & LCM60 & $4147-57-3$ & 2.8 & $\mathrm{ng} / \mathrm{L}$ & RLDQC \\
\hline Demethyl fluometuron & 68591 & LCM60 & $3032-40-4$ & 3.6 & $\mathrm{ng} / \mathrm{L}$ & RLDQC \\
\hline Demethyl hexazinone B & 68566 & LCM60 & $56611-54-2$ & 3 & $\mathrm{ng} / \mathrm{L}$ & RLDQC \\
\hline Demethyl norflurazon & 68567 & LCM60 & $23576-24-1$ & 4 & $\mathrm{ng} / \mathrm{L}$ & RLDQC \\
\hline
\end{tabular}


Table 2.3.1. U.S. Geological National Water Quality Laboratory Schedule 2437 for current-use pesticides in filtered water.-Continued

[Methods are described in Sandstrom and others (2016). Parameter names are as shown in the U.S. Geological Survey (USGS) National Water Information System (NWIS) database and do not include special characters or formatting. Abbreviations: CAS, Chemical Abstracts Service'; N/A, not applicable; ng/L, nanogram per liter; NWQL, USGS National Water Quality Laboratory; pct, percent; RLDQC, reporting level based on DQCALC software and procedure, as described in NWQL Technical Memorandum 15.02; - , no data]

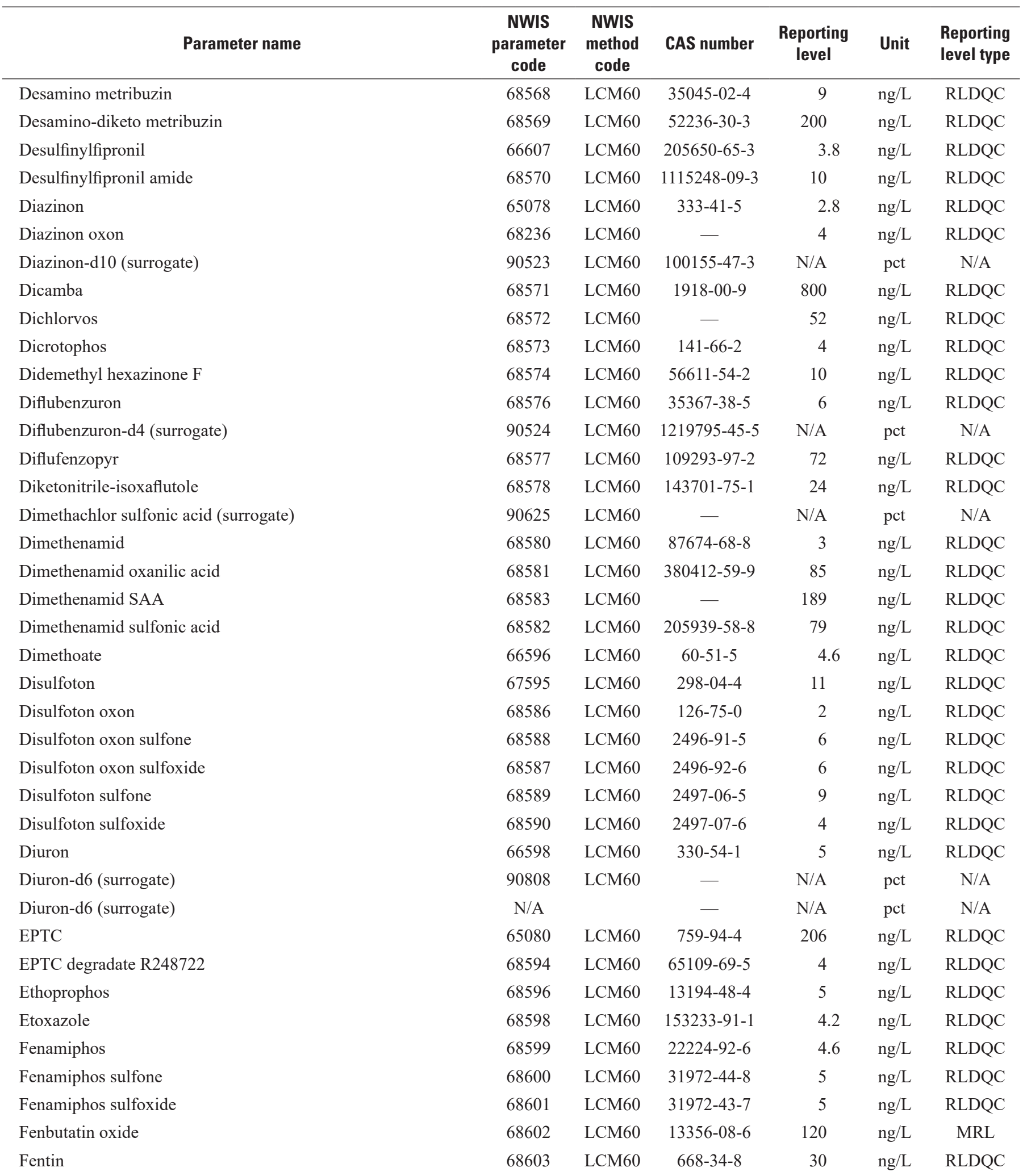


Table 2.3.1. U.S. Geological National Water Quality Laboratory Schedule 2437 for current-use pesticides in filtered water-—Continued

[Methods are described in Sandstrom and others (2016). Parameter names are as shown in the U.S. Geological Survey (USGS) National Water Information System (NWIS) database and do not include special characters or formatting. Abbreviations: CAS, Chemical Abstracts Service ${ }^{1}$; N/A, not applicable; ng/L, nanogram per liter; NWQL, USGS National Water Quality Laboratory; pct, percent; RLDQC, reporting level based on DQCALC software and procedure, as described in NWQL Technical Memorandum 15.02; - , no data]

\begin{tabular}{|c|c|c|c|c|c|c|}
\hline Parameter name & $\begin{array}{c}\text { NWIS } \\
\text { parameter } \\
\text { code }\end{array}$ & $\begin{array}{c}\text { NWIS } \\
\text { method } \\
\text { code }\end{array}$ & CAS number & $\begin{array}{c}\text { Reporting } \\
\text { level }\end{array}$ & Unit & $\begin{array}{l}\text { Reporting } \\
\text { level type }\end{array}$ \\
\hline Fipronil & 66604 & LCM60 & $120068-37-3$ & 4 & $\mathrm{ng} / \mathrm{L}$ & RLDQC \\
\hline Fipronil amide & 68604 & LCM60 & $205650-69-7$ & 9.2 & $\mathrm{ng} / \mathrm{L}$ & RLDQC \\
\hline Fipronil sulfide & 66610 & LCM60 & $120067-83-6$ & 4.2 & $\mathrm{ng} / \mathrm{L}$ & RLDQC \\
\hline Fipronil sulfonate & 68605 & LCM60 & $209248-72-6$ & 96 & $\mathrm{ng} / \mathrm{L}$ & RLDQC \\
\hline Fipronil sulfone & 66613 & LCM60 & $120068-36-2$ & 5.6 & $\mathrm{ng} / \mathrm{L}$ & RLDQC \\
\hline Flubendiamide & 68606 & LCM60 & $272451-65-7$ & 4.4 & $\mathrm{ng} / \mathrm{L}$ & RLDQC \\
\hline Flumetsulam & 61679 & LCM60 & $98967-40-9$ & 17 & $\mathrm{ng} / \mathrm{L}$ & RLDQC \\
\hline Fluometuron & 68608 & LCM60 & $2164-17-2$ & 10 & $\mathrm{ng} / \mathrm{L}$ & RLDQC \\
\hline Fonofos & 65084 & LCM60 & $944-22-9$ & 11 & $\mathrm{ng} / \mathrm{L}$ & RLDQC \\
\hline Halosulfuron-methyl & 61680 & LCM60 & $100784-20-1$ & 12 & $\mathrm{ng} / \mathrm{L}$ & RLDQC \\
\hline Hexazinone & 65085 & LCM60 & $51235-04-2$ & 3.6 & $\mathrm{ng} / \mathrm{L}$ & RLDQC \\
\hline Hexazinone Transformation Product C & 68612 & LCM60 & $72585-88-7$ & 2 & $\mathrm{ng} / \mathrm{L}$ & RLDQC \\
\hline Hexazinone Transformation Product D & 68613 & LCM60 & $30243-77-7$ & 294 & $\mathrm{ng} / \mathrm{L}$ & RLDQC \\
\hline Hexazinone Transformation Product E & 68614 & LCM60 & $72576-14-8$ & 76 & $\mathrm{ng} / \mathrm{L}$ & RLDQC \\
\hline Hexazinone Transformation Product $G$ & 68713 & LCM60 & - & 22 & $\mathrm{ng} / \mathrm{L}$ & RLDQC \\
\hline Hexazinone-d6 (surrogate) & 90527 & LCM60 & $1219804-22-4$ & N/A & pct & N/A \\
\hline Hydroxy didemethyl fluometuron & 68619 & LCM60 & - & 50 & $\mathrm{ng} / \mathrm{L}$ & RLDQC \\
\hline Hydroxy monodemethyl fluometuron & 68617 & LCM60 & - & 12 & $\mathrm{ng} / \mathrm{L}$ & RLDQC \\
\hline Hydroxyacetochlor & 68615 & LCM60 & $60090-47-3$ & 20 & $\mathrm{ng} / \mathrm{L}$ & RLDQC \\
\hline Hydroxyalachlor & 68616 & LCM60 & $56681-55-1$ & 6 & $\mathrm{ng} / \mathrm{L}$ & RLDQC \\
\hline Hydroxydiazinon & 68618 & LCM60 & $29820-16-4$ & 11 & $\mathrm{ng} / \mathrm{L}$ & RLDQC \\
\hline Hydroxyfluometuron & 68620 & LCM60 & - & 56 & $\mathrm{ng} / \mathrm{L}$ & RLDQC \\
\hline Hydroxymetolachlor & 68622 & LCM60 & $131068-72-9$ & 2.4 & $\mathrm{ng} / \mathrm{L}$ & RLDQC \\
\hline Hydroxyphthalazinone & 68623 & LCM60 & - & 28 & $\mathrm{ng} / \mathrm{L}$ & RLDQC \\
\hline Hydroxysimazine & 68624 & LCM60 & $2599-11-3$ & 120 & $\mathrm{ng} / \mathrm{L}$ & RLDQC \\
\hline Imazamox & 68625 & LCM60 & $114311-32-9$ & 30 & $\mathrm{ng} / \mathrm{L}$ & RLDQC \\
\hline Imazaquin & 61682 & LCM60 & $81335-37-7$ & 18 & $\mathrm{ng} / \mathrm{L}$ & RLDQC \\
\hline Imazethapyr & 61683 & LCM60 & $81335-77-5$ & 8 & $\mathrm{ng} / \mathrm{L}$ & RLDQC \\
\hline Imidacloprid & 68426 & LCM60 & $138261-41-3$ & 16 & $\mathrm{ng} / \mathrm{L}$ & RLDQC \\
\hline Indoxacarb & 68627 & LCM60 & $173584-44-6$ & 5.2 & $\mathrm{ng} / \mathrm{L}$ & RLDQC \\
\hline Isoxaflutole & 68632 & LCM60 & $141112-29-0$ & 18 & $\mathrm{ng} / \mathrm{L}$ & RLDQC \\
\hline Isoxaflutole acid metabolite RPA 203328 & 68633 & LCM60 & $142994-06-7$ & 9.2 & $\mathrm{ng} / \mathrm{L}$ & RLDQC \\
\hline Kresoxim-methyl & 67670 & LCM60 & $143390-89-0$ & 5 & $\mathrm{ng} / \mathrm{L}$ & RLDQC \\
\hline Lactofen & 68638 & LCM60 & $77501-63-4$ & 10 & $\mathrm{ng} / \mathrm{L}$ & RLDQC \\
\hline Linuron & 68639 & LCM60 & $330-55-2$ & 5.6 & $\mathrm{ng} / \mathrm{L}$ & RLDQC \\
\hline Linuron-d6 (dimethyl-d6) (surrogate) & 90529 & LCM60 & $1219804-76-8$ & N/A & pct & N/A \\
\hline Linuron-d6 (dimethyl-d6) (surrogate) & N/A & & $1219804-76-8$ & N/A & pct & N/A \\
\hline Malaoxon & 68240 & LCM60 & $1634-78-2$ & 2.4 & $\mathrm{ng} / \mathrm{L}$ & RLDQC \\
\hline Malathion & 65087 & LCM60 & $121-75-5$ & 5.4 & $\mathrm{ng} / \mathrm{L}$ & RLDQC \\
\hline
\end{tabular}


Table 2.3.1. U.S. Geological National Water Quality Laboratory Schedule 2437 for current-use pesticides in filtered water.-Continued

[Methods are described in Sandstrom and others (2016). Parameter names are as shown in the U.S. Geological Survey (USGS) National Water Information System (NWIS) database and do not include special characters or formatting. Abbreviations: CAS, Chemical Abstracts Service'; N/A, not applicable; ng/L, nanogram per liter; NWQL, USGS National Water Quality Laboratory; pct, percent; RLDQC, reporting level based on DQCALC software and procedure, as described in NWQL Technical Memorandum 15.02; - , no data]

\begin{tabular}{|c|c|c|c|c|c|c|}
\hline Parameter name & $\begin{array}{c}\text { NWIS } \\
\text { parameter } \\
\text { code }\end{array}$ & $\begin{array}{l}\text { NWIS } \\
\text { method } \\
\text { code }\end{array}$ & CAS number & $\begin{array}{c}\text { Reporting } \\
\text { level }\end{array}$ & Unit & $\begin{array}{l}\text { Reporting } \\
\text { level type }\end{array}$ \\
\hline Malathion-d10 (diethyl-d10) (surrogate) & 90552 & LCM60 & $347841-48-9$ & $\mathrm{~N} / \mathrm{A}$ & pct & N/A \\
\hline MCPA & 68641 & LCM60 & $94-74-6$ & 95 & $\mathrm{ng} / \mathrm{L}$ & RLDQC \\
\hline Metalaxyl & 68437 & LCM60 & $57837-19-1$ & 6 & $\mathrm{ng} / \mathrm{L}$ & RLDQC \\
\hline Methidathion & 65088 & LCM60 & $950-37-8$ & 8.4 & $\mathrm{ng} / \mathrm{L}$ & RLDQC \\
\hline Methomyl & 68645 & LCM60 & $16752-77-5$ & 3 & $\mathrm{ng} / \mathrm{L}$ & RLDQC \\
\hline Methomyl oxime & 68646 & LCM60 & $13749-94-5$ & 8,000 & $\mathrm{ng} / \mathrm{L}$ & RLDQC \\
\hline Methoxyfenozide & 68647 & LCM60 & $161050-58-4$ & 2.2 & $\mathrm{ng} / \mathrm{L}$ & RLDQC \\
\hline Metolachlor oxanilic acid & 68650 & LCM60 & $152019-73-3$ & 149 & $\mathrm{ng} / \mathrm{L}$ & RLDQC \\
\hline Metolachlor sulfonic acid & 68651 & LCM60 & $171118-09-5$ & 68 & $\mathrm{ng} / \mathrm{L}$ & RLDQC \\
\hline Metolachlor-d6 (propyl-d6) (surrogate) & 90553 & LCM60 & $1219803-97-0$ & N/A & pet & N/A \\
\hline Metribuzin & 68652 & LCM60 & $21087-64-9$ & 20 & $\mathrm{ng} / \mathrm{L}$ & RLDQC \\
\hline Metribuzin DK & 68653 & LCM60 & $56507-37-0$ & 236 & $\mathrm{ng} / \mathrm{L}$ & RLDQC \\
\hline Molinate & 65091 & LCM60 & $2212-67-1$ & 28 & $\mathrm{ng} / \mathrm{L}$ & RLDQC \\
\hline Myclobutanil & 66632 & LCM60 & $88671-89-0$ & 7 & $\mathrm{ng} / \mathrm{L}$ & RLDQC \\
\hline N-(3,4-Dichlorophenyl)-N'-methylurea & 68231 & LCM60 & $3567-62-2$ & 5 & $\mathrm{ng} / \mathrm{L}$ & RLDQC \\
\hline Naled & 68654 & LCM60 & $300-76-5$ & 56 & $\mathrm{ng} / \mathrm{L}$ & RLDQC \\
\hline O-Ethyl-S-propyl phosphorothioate & 68658 & LCM60 & $31110-62-0$ & 64 & $\mathrm{ng} / \mathrm{L}$ & RLDQC \\
\hline Omethoate (Dimethoate oxon) & 68661 & LCM60 & $1113-02-6$ & 2 & $\mathrm{ng} / \mathrm{L}$ & RLDQC \\
\hline Orthosulfamuron & 68662 & LCM60 & 213464-77-8 & 6 & $\mathrm{ng} / \mathrm{L}$ & RLDQC \\
\hline Oryzalin & 68663 & LCM60 & $19044-88-3$ & 12 & $\mathrm{ng} / \mathrm{L}$ & RLDQC \\
\hline Oxamyl & 68664 & LCM60 & $23135-22-0$ & 2 & $\mathrm{ng} / \mathrm{L}$ & RLDQC \\
\hline Oxamyl oxime & 68665 & LCM60 & $30558-43-1$ & 5 & $\mathrm{ng} / \mathrm{L}$ & RLDQC \\
\hline Oxyfluorfen & 65093 & LCM60 & $42874-03-3$ & 500 & $\mathrm{ng} / \mathrm{L}$ & RLDQC \\
\hline Paraoxon & 68666 & LCM60 & $311-45-5$ & 3.4 & $\mathrm{ng} / \mathrm{L}$ & RLDQC \\
\hline Pendimethalin & 65098 & LCM60 & $40487-42-1$ & 10 & $\mathrm{ng} / \mathrm{L}$ & RLDQC \\
\hline Phorate & 68668 & LCM60 & $298-02-2$ & 11 & $\mathrm{ng} / \mathrm{L}$ & RLDQC \\
\hline Phorate oxon & 68669 & LCM60 & $2600-69-3$ & 55 & $\mathrm{ng} / \mathrm{L}$ & RLDQC \\
\hline Phorate oxon sulfone & 68670 & LCM60 & $2588-06-9$ & 20 & $\mathrm{ng} / \mathrm{L}$ & RLDQC \\
\hline
\end{tabular}


Table 2.3.1. U.S. Geological National Water Quality Laboratory Schedule 2437 for current-use pesticides in filtered water-—Continued

[Methods are described in Sandstrom and others (2016). Parameter names are as shown in the U.S. Geological Survey (USGS) National Water Information System (NWIS) database and do not include special characters or formatting. Abbreviations: CAS, Chemical Abstracts Service'; N/A, not applicable; ng/L, nanogram per liter; NWQL, USGS National Water Quality Laboratory; pct, percent; RLDQC, reporting level based on DQCALC software and procedure, as described in NWQL Technical Memorandum 15.02; - , no data]

\begin{tabular}{|c|c|c|c|c|c|c|}
\hline Parameter name & $\begin{array}{c}\text { NWIS } \\
\text { parameter } \\
\text { code }\end{array}$ & $\begin{array}{c}\text { NWIS } \\
\text { method } \\
\text { code }\end{array}$ & CAS number & $\begin{array}{c}\text { Reporting } \\
\text { level }\end{array}$ & Unit & $\begin{array}{l}\text { Reporting } \\
\text { level type }\end{array}$ \\
\hline Phorate oxon sulfoxide & 68671 & LCM60 & $2588-05-8$ & 7 & $\mathrm{ng} / \mathrm{L}$ & RLDQC \\
\hline Phorate sulfone & 68672 & LCM60 & $2588-04-7$ & 36 & $\mathrm{ng} / \mathrm{L}$ & RLDQC \\
\hline Phorate sulfoxide & 68673 & LCM60 & $2588-03-6$ & 4.6 & $\mathrm{ng} / \mathrm{L}$ & RLDQC \\
\hline Phthalazinone & 68675 & LCM60 & $90004-07-2$ & 15 & $\mathrm{ng} / \mathrm{L}$ & RLDQC \\
\hline Piperonyl butoxide & 65102 & LCM60 & $51-03-6$ & 60 & $\mathrm{ng} / \mathrm{L}$ & RLDQC \\
\hline Profenofos & 68676 & LCM60 & 41198-08-7 & 3 & $\mathrm{ng} / \mathrm{L}$ & RLDQC \\
\hline Prometon & 67702 & LCM60 & $1610-18-0$ & 4 & $\mathrm{ng} / \mathrm{L}$ & RLDQC \\
\hline Prometryn & 65103 & LCM60 & $7287-19-6$ & 4.2 & $\mathrm{ng} / \mathrm{L}$ & RLDQC \\
\hline Propanil & 66641 & LCM60 & $709-98-8$ & 12 & $\mathrm{ng} / \mathrm{L}$ & RLDQC \\
\hline Propargite & 68677 & LCM60 & $2312-35-8$ & 2 & $\mathrm{ng} / \mathrm{L}$ & RLDQC \\
\hline Propazine & 68678 & LCM60 & $139-40-2$ & 3.2 & $\mathrm{ng} / \mathrm{L}$ & RLDQC \\
\hline Propiconazole & 66643 & LCM60 & $60207-90-1$ & 6 & $\mathrm{ng} / \mathrm{L}$ & RLDQC \\
\hline Propoxur & 68679 & LCM60 & $114-26-1$ & 3.2 & $\mathrm{ng} / \mathrm{L}$ & RLDQC \\
\hline Propyzamide & 67706 & LCM60 & $23950-58-5$ & 2.4 & $\mathrm{ng} / \mathrm{L}$ & RLDQC \\
\hline Prosulfuron & 61687 & LCM60 & $94125-34-5$ & 10 & $\mathrm{ng} / \mathrm{L}$ & RLDQC \\
\hline Pyraclostrobin & 66646 & LCM60 & $175013-18-0$ & 2.4 & $\mathrm{ng} / \mathrm{L}$ & RLDQC \\
\hline Pyridaben & 68682 & LCM60 & $96489-71-3$ & 2.4 & $\mathrm{ng} / \mathrm{L}$ & RLDQC \\
\hline Pyriproxyfen & 68683 & LCM60 & $95737-68-1$ & 3 & $\mathrm{ng} / \mathrm{L}$ & RLDQC \\
\hline sec-Acetochlor oxanilic acid & 68684 & LCM60 & $152019-74-4$ & 55 & $\mathrm{ng} / \mathrm{L}$ & RLDQC \\
\hline sec-Alachlor oxanilic acid & 68685 & LCM60 & $628324-79-8$ & 110 & $\mathrm{ng} / \mathrm{L}$ & RLDQC \\
\hline Siduron & 68686 & LCM60 & $1982-49-6$ & 5 & $\mathrm{ng} / \mathrm{L}$ & RLDQC \\
\hline Simazine & 65105 & LCM60 & $122-34-9$ & 7.2 & $\mathrm{ng} / \mathrm{L}$ & RLDQC \\
\hline Sulfentrazone & 68687 & LCM60 & $122836-35-5$ & 18 & $\mathrm{ng} / \mathrm{L}$ & RLDQC \\
\hline Sulfometuron-methyl & 68688 & LCM60 & $74222-97-2$ & 4 & $\mathrm{ng} / \mathrm{L}$ & RLDQC \\
\hline Sulfosulfuron & 68689 & LCM60 & $141776-32-1$ & 11 & $\mathrm{ng} / \mathrm{L}$ & RLDQC \\
\hline Sulfosulfuron ethyl sulfone & 68690 & LCM60 & - & 2.8 & $\mathrm{ng} / \mathrm{L}$ & RLDQC \\
\hline Tebuconazole & 66649 & LCM60 & $107534-96-3$ & 15 & $\mathrm{ng} / \mathrm{L}$ & RLDQC \\
\hline Tebuconazole-d6 (surrogate) & 90555 & LCM60 & - & $\mathrm{N} / \mathrm{A}$ & pct & $\mathrm{N} / \mathrm{A}$ \\
\hline Tebufenozide & 68692 & LCM60 & $112410-23-8$ & 2 & $\mathrm{ng} / \mathrm{L}$ & RLDQC \\
\hline Tebupirimfos oxon & 68694 & LCM60 & - & 2 & $\mathrm{ng} / \mathrm{L}$ & RLDQC \\
\hline Tebupirimphos & 68693 & LCM60 & $96182-53-5$ & 2 & $\mathrm{ng} / \mathrm{L}$ & RLDQC \\
\hline Tebuthiuron & 68695 & LCM60 & $34014-18-1$ & 3 & $\mathrm{ng} / \mathrm{L}$ & RLDQC \\
\hline Tebuthiuron TP 104 & 68575 & LCM60 & $59962-53-7$ & 5.6 & $\mathrm{ng} / \mathrm{L}$ & RLDQC \\
\hline Tebuthiuron TP 109 & 68621 & LCM60 & $59962-54-8$ & 11 & $\mathrm{ng} / \mathrm{L}$ & RLDQC \\
\hline Tebuthiuron TP $109(\mathrm{OH})$ & 68697 & LCM60 & $139888-73-6$ & 38 & $\mathrm{ng} / \mathrm{L}$ & RLDQC \\
\hline Tebuthiuron TP el108 & 68696 & LCM60 & $39222-73-6$ & 10 & $\mathrm{ng} / \mathrm{L}$ & RLDQC \\
\hline Tebuthiuron Transformation Product 106 & 68714 & LCM60 & $16279-27-9$ & 32 & $\mathrm{ng} / \mathrm{L}$ & RLDQC \\
\hline Terbacil & 68698 & LCM60 & $5902-51-2$ & 21 & $\mathrm{ng} / \mathrm{L}$ & RLDQC \\
\hline Terbufos & 68699 & LCM60 & $13071-79-9$ & 6.8 & $\mathrm{ng} / \mathrm{L}$ & RLDQC \\
\hline
\end{tabular}


Table 2.3.1. U.S. Geological National Water Quality Laboratory Schedule 2437 for current-use pesticides in filtered water.-Continued

[Methods are described in Sandstrom and others (2016). Parameter names are as shown in the U.S. Geological Survey (USGS) National Water Information System (NWIS) database and do not include special characters or formatting. Abbreviations: CAS, Chemical Abstracts Service1; N/A, not applicable; ng/L, nanogram per liter; NWQL, USGS National Water Quality Laboratory; pct, percent; RLDQC, reporting level based on DQCALC software and procedure, as described in NWQL Technical Memorandum 15.02; - , no data]

\begin{tabular}{|c|c|c|c|c|c|c|}
\hline Parameter name & $\begin{array}{c}\text { NWIS } \\
\text { parameter } \\
\text { code }\end{array}$ & $\begin{array}{c}\text { NWIS } \\
\text { method } \\
\text { code }\end{array}$ & CAS number & $\begin{array}{c}\text { Reporting } \\
\text { level }\end{array}$ & Unit & $\begin{array}{l}\text { Reporting } \\
\text { level type }\end{array}$ \\
\hline Terbufos oxon & 68700 & LCM60 & $56070-14-5$ & 4 & $\mathrm{ng} / \mathrm{L}$ & RLDQC \\
\hline Terbufos oxon sulfone & 68701 & LCM60 & $56070-15-6$ & 11 & $\mathrm{ng} / \mathrm{L}$ & RLDQC \\
\hline Terbufos oxon sulfoxide & 68702 & LCM60 & $56165-57-2$ & 4 & $\mathrm{ng} / \mathrm{L}$ & RLDQC \\
\hline Terbuthylazine & 66651 & LCM60 & $5915-41-3$ & 3.6 & $\mathrm{ng} / \mathrm{L}$ & RLDQC \\
\hline Tetraconazole & 66654 & LCM60 & $112281-77-3$ & 7 & $\mathrm{ng} / \mathrm{L}$ & RLDQC \\
\hline Thiobencarb & 65107 & LCM60 & $28249-77-6$ & 4.2 & $\mathrm{ng} / \mathrm{L}$ & RLDQC \\
\hline Thiobencarb-d10 (surrogate) & 90556 & LCM60 & $1219804-12-2$ & N/A & pct & $\mathrm{N} / \mathrm{A}$ \\
\hline Triclopyr & 68712 & LCM60 & $55335-06-3$ & 88 & $\mathrm{ng} / \mathrm{L}$ & RLDQC \\
\hline Trifloxystrobin & 66660 & LCM60 & $141517-21-7$ & 2.8 & $\mathrm{ng} / \mathrm{L}$ & RLDQC \\
\hline
\end{tabular}

${ }^{1}$ This report contains CAS Registry Numbers ${ }^{\circledR}$, which is a Registered Trademark of the American Chemical Society. CAS recommends the verification of the CAS Registry Numbers through CAS Client Services ${ }^{\mathrm{SM}}$. 
Table 2.3.2. U.S. Geological National Water Quality Laboratory custom method LCM75 for supplemental pesticides and degradates in filtered water.

[Method is equivalent to that in Sandstrom and others (2016), except that the instrument used is an Agilent model 6495 LC-MS/MS. Parameter names are as shown in the U.S. Geological Survey (USGS) National Water Information System (NWIS) database and do not include special characters or formatting. Abbrteviations: CAS, Chemical Abstracts Service1; irl, interim reporting level; N/A, not applicable; ng/L, nanogram per liter; NWQL, USGS National Water Quality Laboratory; pct, percent; RSD, relative standard deviation; - , no data]

\begin{tabular}{|c|c|c|c|c|c|c|c|c|c|}
\hline Parameter name & $\begin{array}{l}\text { NWOL } \\
\text { lab } \\
\text { code }\end{array}$ & $\begin{array}{c}\text { NWIS } \\
\text { parameter } \\
\text { code }\end{array}$ & $\begin{array}{l}\text { NWIS } \\
\text { method } \\
\text { code }\end{array}$ & CAS number & $\begin{array}{l}\text { Reporting } \\
\text { level }\end{array}$ & Unit & $\begin{array}{l}\text { Reporting } \\
\text { level type }\end{array}$ & $\begin{array}{c}\text { Mean } \\
\text { recovery } \\
\text { (percent) }\end{array}$ & $\begin{array}{c}\text { RSD } \\
\text { (percent) }\end{array}$ \\
\hline 2,6-Dichlorobenzamide & 9045 & 52656 & LCM75 & $2008-58-4$ & 3.3 & $\mathrm{ng} / \mathrm{L}$ & irl & 106.4 & 13.2 \\
\hline Acetamiprid & 9045 & 68302 & LCM75 & 135410-20-7 & 11 & $\mathrm{ng} / \mathrm{L}$ & irl & 105.5 & 10.2 \\
\hline Acetamiprid-13C6 (surrogate) & 9045 & 90453 & LCM75 & - & $\mathrm{N} / \mathrm{A}$ & pct & irl & - & - \\
\hline Aminocyclopyrachlor & 9045 & 52651 & LCM75 & 858956-08-8 & 2.2 & $\mathrm{ng} / \mathrm{L}$ & irl & 113.2 & 16.3 \\
\hline Benzovindiflupyr & 9045 & 52652 & LCM75 & 1072957-71-1 & 2.2 & $\mathrm{ng} / \mathrm{L}$ & irl & 103.2 & 10.3 \\
\hline Bicyclopyrone & 9045 & 52653 & LCM75 & $352010-68-5$ & 5.2 & $\mathrm{ng} / \mathrm{L}$ & irl & 106.6 & 10.6 \\
\hline Boscalid & 9045 & 67550 & LCM75 & $188425-85-6$ & 3.9 & $\mathrm{ng} / \mathrm{L}$ & irl & 108.2 & 10.5 \\
\hline Chlorantraniliprole & 9045 & 51856 & LCM75 & $500008-45-7$ & 3 & $\mathrm{ng} / \mathrm{L}$ & irl & 107.0 & 12.2 \\
\hline Clothianidin & 9045 & 68221 & LCM75 & $210880-92-5$ & 6 & $\mathrm{ng} / \mathrm{L}$ & irl & 105.5 & 11.0 \\
\hline $\begin{array}{l}\text { N-(2-Chlorothiazol-5-ylmethy1)- } \\
\text { N'-methylguanidine (TMG) }\end{array}$ & 9045 & 52661 & LCM75 & 635283-91-9 & 3.9 & $\mathrm{ng} / \mathrm{L}$ & irl & 118.1 & 11.1 \\
\hline $\begin{array}{l}\text { N-(2-Chloro-5-thiazolyl methyl)- } \\
\text { N'-nitroguanidine (TZNG) }\end{array}$ & 9045 & 52660 & LCM75 & $135018-15-4$ & 19 & $\mathrm{ng} / \mathrm{L}$ & irl & 102.0 & 11.3 \\
\hline Clothianidin-d3 (surrogate) & 9045 & 90452 & LCM75 & $1262776-24-8$ & $\mathrm{~N} / \mathrm{A}$ & pct & irl & - & - \\
\hline Cyantraniliprole & 9045 & 51862 & LCM75 & 736994-63-1 & 4.6 & $\mathrm{ng} / \mathrm{L}$ & irl & 83.7 & 14.8 \\
\hline $\begin{array}{l}\text { Cyantraniliprole degradate IN- } \\
\text { JSE76 }\end{array}$ & 9045 & 52662 & LCM75 & - & 7 & $\mathrm{ng} / \mathrm{L}$ & irl & 101.7 & 15.0 \\
\hline $\begin{array}{l}\text { Cyantraniliprole degradate IN- } \\
\text { K5A79 }\end{array}$ & 9045 & 52663 & LCM75 & - & 13 & $\mathrm{ng} / \mathrm{L}$ & irl & 86.7 & 18.9 \\
\hline $\begin{array}{l}\text { Cyantraniliprole degradate IN- } \\
\text { RNU71 }\end{array}$ & 9045 & 52664 & LCM75 & - & 14 & $\mathrm{ng} / \mathrm{L}$ & irl & 104.5 & 13.7 \\
\hline Cyprodinil & 9045 & 67574 & LCM75 & 121552-61-2 & 7.6 & $\mathrm{ng} / \mathrm{L}$ & irl & 105.0 & 10.3 \\
\hline Dimethomorph & 9045 & 68373 & LCM75 & $110488-70-5$ & 2.9 & $\mathrm{ng} / \mathrm{L}$ & irl & 104.4 & 10.1 \\
\hline Dinotefuran & 9045 & 68379 & LCM75 & $165252-70-0$ & 2.8 & $\mathrm{ng} / \mathrm{L}$ & irl & 106.4 & 11.3 \\
\hline Fluopicolide & 9045 & 51852 & LCM75 & $239110-15-7$ & 3 & $\mathrm{ng} / \mathrm{L}$ & irl & 106.0 & 9.6 \\
\hline Flutriafol & 9045 & 67653 & LCM75 & $76674-21-0$ & 2.2 & $\mathrm{ng} / \mathrm{L}$ & irl & 103.8 & 18.9 \\
\hline Fomesafen & 9045 & 52654 & LCM75 & 72178-02-0 & 7.5 & $\mathrm{ng} / \mathrm{L}$ & irl & 104.3 & 12.0 \\
\hline Imazosulfuron & 9045 & 52655 & LCM75 & $122548-33-8$ & 4.1 & $\mathrm{ng} / \mathrm{L}$ & irl & 105.4 & 11.4 \\
\hline Imidacloprid & 9045 & 68426 & LCM75 & 138261-41-3 & 14 & $\mathrm{ng} / \mathrm{L}$ & irl & 107.7 & 13.3 \\
\hline Imidacloprid-d4 (surrogate) & 9045 & 90537 & LCM75 & $1015855-75-0$ & N/A & pet & irl & - & - \\
\hline Imidacloprid-olefin & 9045 & 52782 & LCM75 & 115086-54-9 & 8.9 & $\mathrm{ng} / \mathrm{L}$ & irl & 115.4 & 9.5 \\
\hline Myclobutanil & 9045 & 66632 & LCM75 & $88671-89-0$ & 2.7 & $\mathrm{ng} / \mathrm{L}$ & irl & 106.8 & 10.4 \\
\hline Pyrimethanil & 9045 & 67717 & LCM75 & $53112-28-0$ & 9 & $\mathrm{ng} / \mathrm{L}$ & irl & 107.0 & 12.9 \\
\hline Sulfoxaflor & 9045 & 52657 & LCM75 & $946578-00-3$ & 3.1 & $\mathrm{ng} / \mathrm{L}$ & irl & 109.6 & 9.8 \\
\hline Tebuconazole-d6 (surrogate) & 9045 & 90555 & LCM75 & - & N/A & pct & irl & - & - \\
\hline Thiacloprid & 9045 & 68485 & LCM75 & $111988-49-9$ & 3.3 & $\mathrm{ng} / \mathrm{L}$ & irl & 106.4 & 10.4 \\
\hline Thiamethoxam & 9045 & 68245 & LCM75 & 153719-23-4 & 2.8 & $\mathrm{ng} / \mathrm{L}$ & irl & 109.0 & 13.1 \\
\hline Thiamethoxam-d3 (surrogate) & 9045 & 90451 & LCM75 & $1294048-82-0$ & & pct & irl & - & - \\
\hline Tribenuron-methyl & 9045 & 52650 & LCM75 & $101200-48-0$ & 56 & $\mathrm{ng} / \mathrm{L}$ & irl & 34.3 & 73.1 \\
\hline Zoxamide & 9045 & 67768 & LCM75 & $156052-68-5$ & 2.3 & $\mathrm{ng} / \mathrm{L}$ & irl & 102.8 & 9.4 \\
\hline
\end{tabular}

${ }^{1}$ This report contains CAS Registry Numbers ${ }^{\circledR}$, which is a Registered Trademark of the American Chemical Society. CAS recommends the verification of the CAS Registry Numbers through CAS Client Services ${ }^{S M}$. 
Table 2.4. U.S. Geological Survey National Water Quality Laboratory Schedule 2440 for pharmaceutical compounds in water.

[Methods are described in Furlong and others (2014). Parameter names are as shown in the U.S. Geological Survey (USGS) National Water Information System (NWIS) database and do not include special characters or formatting. Abbreviations: CAS, Chemical Abstracts Service ${ }^{1}$; irl, interim reporting level; mrl, minimum reporting level; N/A, not applicable; ng/L, nanogram per liter; NWQL, USGS National Water Quality Laboratory; pct, percent; - , no data]

\begin{tabular}{|c|c|c|c|c|c|c|}
\hline Parameter name & $\begin{array}{c}\text { NWIS } \\
\text { parameter } \\
\text { code }\end{array}$ & $\begin{array}{c}\text { NWIS } \\
\text { method } \\
\text { code }\end{array}$ & CAS number & $\begin{array}{c}\text { Reporting } \\
\text { level }\end{array}$ & Unit & $\begin{array}{l}\text { Reporting } \\
\text { level type }\end{array}$ \\
\hline 1,7-Dimethylxanthine (p-Xanthine) & 67446 & LCM56 & $611-59-6$ & 87 & $\mathrm{ng} / \mathrm{L}$ & $\operatorname{irl}$ \\
\hline 10-Hydroxy-amitriptyline & 67995 & LCM56 & $64520-05-4$ & 8.3 & $\mathrm{ng} / \mathrm{L}$ & $\operatorname{irl}$ \\
\hline Abacavir & 68022 & LCM56 & $136470-78-5$ & 8.2 & $\mathrm{ng} / \mathrm{L}$ & $\operatorname{irl}$ \\
\hline Acetaminophen & 67436 & LCM56 & $103-90-2$ & 7.1 & $\mathrm{ng} / \mathrm{L}$ & irl \\
\hline Acyclovir & 67484 & LCM56 & $59277-89-3$ & 22 & $\mathrm{ng} / \mathrm{L}$ & $\operatorname{irl}$ \\
\hline Albuterol & 67437 & LCM56 & 18559-94-9 & 6.7 & $\mathrm{ng} / \mathrm{L}$ & $\operatorname{irl}$ \\
\hline Albuterol-d9 (surrogate) & 91772 & LCM56 & - & $\mathrm{N} / \mathrm{A}$ & pct & $\mathrm{mrl}$ \\
\hline Alprazolam & 68250 & LCM56 & 28981-97-7 & 21 & $\mathrm{ng} / \mathrm{L}$ & $\operatorname{irl}$ \\
\hline Antipyrine & 67477 & LCM56 & $60-80-0$ & 116 & $\mathrm{ng} / \mathrm{L}$ & $\operatorname{irl}$ \\
\hline Atenolol & 67502 & LCM56 & $29122-68-7$ & 13 & $\mathrm{ng} / \mathrm{L}$ & $\operatorname{irl}$ \\
\hline Atrazine & 65065 & LCM56 & $1912-24-9$ & 19 & $\mathrm{ng} / \mathrm{L}$ & $\operatorname{irl}$ \\
\hline Benztropine & 67997 & LCM56 & $86-13-5$ & 24 & $\mathrm{ng} / \mathrm{L}$ & $\operatorname{irl}$ \\
\hline Betamethasone & 67485 & LCM56 & $378-44-9$ & 114 & $\mathrm{ng} / \mathrm{L}$ & irl \\
\hline Bupropion & 67439 & LCM56 & $34911-55-2$ & 17 & $\mathrm{ng} / \mathrm{L}$ & $\operatorname{irl}$ \\
\hline Caffeine & 67440 & LCM56 & $58-08-2$ & 90 & $\mathrm{ng} / \mathrm{L}$ & irl \\
\hline Caffeine (trimethyl-13C3) (surrogate) & 91781 & LCM56 & - & N/A & pct & $\mathrm{mrl}$ \\
\hline Carbamazepine & 67441 & LCM56 & $298-46-4$ & 11 & $\mathrm{ng} / \mathrm{L}$ & $\operatorname{irl}$ \\
\hline Codeine-d6 (surrogate) & 91786 & LCM56 & $371151-94-9$ & $\mathrm{~N} / \mathrm{A}$ & pet & $\mathrm{mrl}$ \\
\hline Cotinine & 67444 & LCM56 & $486-56-6$ & 6.3 & $\mathrm{ng} / \mathrm{L}$ & $\operatorname{irl}$ \\
\hline Cotinine-d3 (surrogate) & 91783 & LCM56 & - & N/A & pet & $\mathrm{mrl}$ \\
\hline Dehydronifedipine & 67445 & LCM56 & $67035-22-7$ & 24 & $\mathrm{ng} / \mathrm{L}$ & $\operatorname{irl}$ \\
\hline Desmethyldiltiazem & 67999 & LCM56 & - & 12 & $\mathrm{ng} / \mathrm{L}$ & $\operatorname{irl}$ \\
\hline Desvenlafaxine & 68251 & LCM56 & $93413-62-8$ & 7.4 & $\mathrm{ng} / \mathrm{L}$ & $\operatorname{irl}$ \\
\hline Dextromethorphan & 67468 & LCM56 & $125-71-3$ & 8.2 & $\mathrm{ng} / \mathrm{L}$ & $\operatorname{irl}$ \\
\hline Diazepam (valium) & 67499 & LCM56 & $439-14-5$ & 2.2 & $\mathrm{ng} / \mathrm{L}$ & irl \\
\hline Diazepam-d5 surrogate (surrogate) & 91790 & LCM56 & $65854-76-4$ & N/A & pet & $\mathrm{mrl}$ \\
\hline Diltiazem & 67519 & LCM56 & $42399-41-7$ & 10 & $\mathrm{ng} / \mathrm{L}$ & $\operatorname{irl}$ \\
\hline Diltiazem-d3 (surrogate) & 91773 & LCM56 & - & N/A & pet & $\mathrm{mrl}$ \\
\hline Diphenhydramine & 67447 & LCM56 & $147-24-0$ & 5.7 & $\mathrm{ng} / \mathrm{L}$ & $\operatorname{irl}$ \\
\hline Diphenhydramine-d3 (surrogate) & 91788 & LCM56 & - & $\mathrm{N} / \mathrm{A}$ & pct & $\mathrm{mrl}$ \\
\hline
\end{tabular}


Table 2.4. U.S. Geological Survey National Water Quality Laboratory Schedule 2440 for pharmaceutical compounds in water.Continued

[Methods are described in Furlong and others (2014). Parameter names are as shown in the U.S. Geological Survey (USGS) National Water Information System (NWIS) database and do not include special characters or formatting. Abbreviations: CAS, Chemical Abstracts Service1; irl, interim reporting level; mrl, minimum reporting level; N/A, not applicable; ng/L, nanogram per liter; NWQL, USGS National Water Quality Laboratory; pct, percent; -, no data]

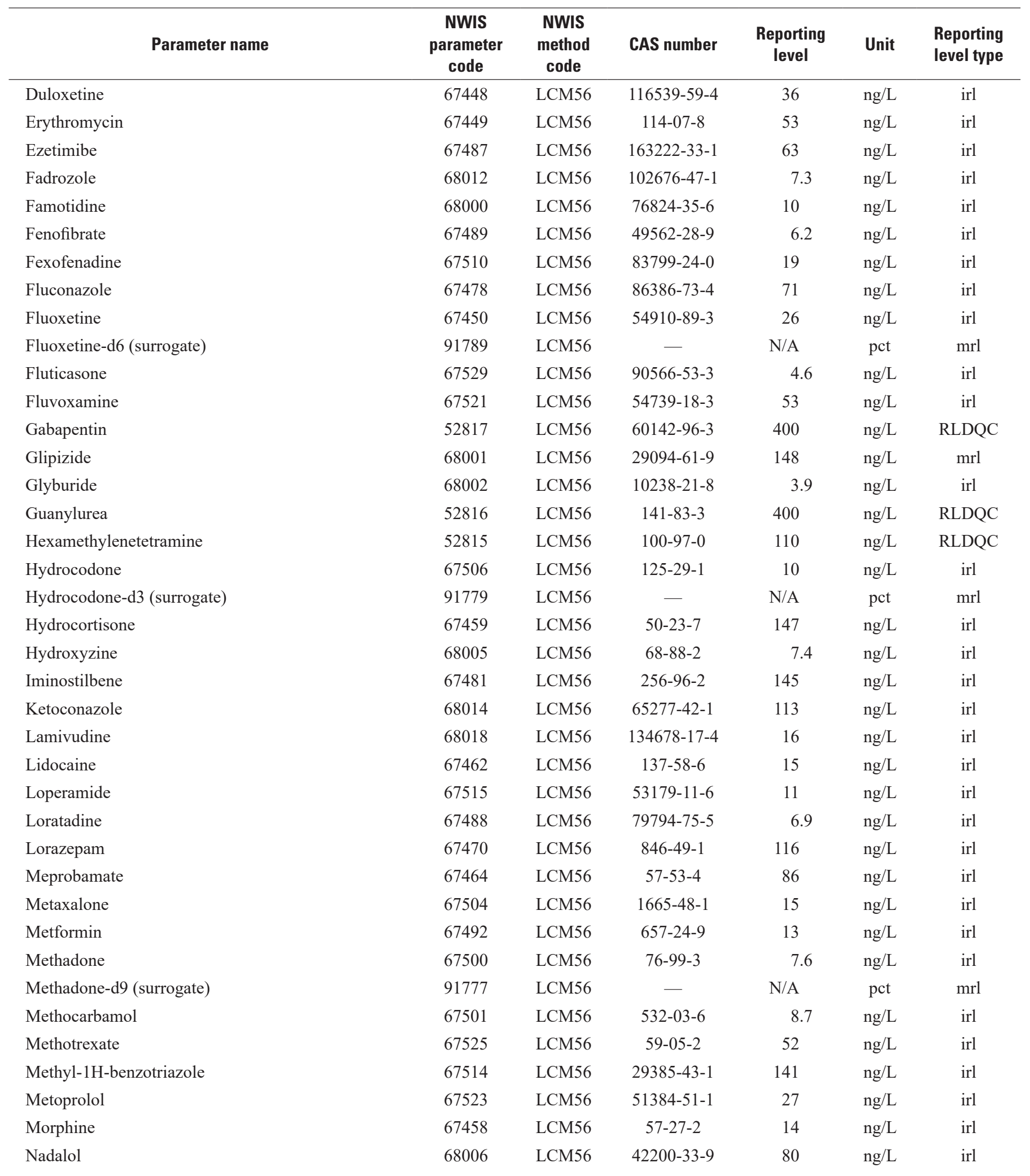


Table 2.4. U.S. Geological Survey National Water Quality Laboratory Schedule 2440 for pharmaceutical compounds in water-— Continued

[Methods are described in Furlong and others (2014). Parameter names are as shown in the U.S. Geological Survey (USGS) National Water Information System (NWIS) database and do not include special characters or formatting. Abbreviations: CAS, Chemical Abstracts Service1; irl, interim reporting level; mrl, minimum reporting level; N/A, not applicable; ng/L, nanogram per liter; NWQL, USGS National Water Quality Laboratory; pct, percent; - , no data]

\begin{tabular}{|c|c|c|c|c|c|c|}
\hline Parameter name & $\begin{array}{c}\text { NWIS } \\
\text { parameter } \\
\text { code }\end{array}$ & $\begin{array}{l}\text { NWIS } \\
\text { method } \\
\text { code }\end{array}$ & CAS number & $\begin{array}{l}\text { Reporting } \\
\text { level }\end{array}$ & Unit & $\begin{array}{l}\text { Reporting } \\
\text { level type }\end{array}$ \\
\hline Nevirapine & 68017 & LCM56 & $129618-40-2$ & 15 & $\mathrm{ng} / \mathrm{L}$ & $\operatorname{irl}$ \\
\hline Nicotine & 67493 & LCM56 & $54-11-5$ & 57 & $\mathrm{ng} / \mathrm{L}$ & $\operatorname{irl}$ \\
\hline Nizatidine & 67479 & LCM56 & $76963-41-2$ & 19 & $\mathrm{ng} / \mathrm{L}$ & $\operatorname{irl}$ \\
\hline Nordiazepam & 68252 & LCM56 & $1088-11-5$ & 41 & $\mathrm{ng} / \mathrm{L}$ & irl \\
\hline Norfluoxetine & 67451 & LCM56 & $56161-73-0$ & 199 & $\mathrm{ng} / \mathrm{L}$ & $\operatorname{irl}$ \\
\hline Norfluoxetine-d6 (surrogate) & 91776 & LCM56 & - & N/A & pct & $\mathrm{mrl}$ \\
\hline Norsertraline & 67532 & LCM56 & $87857-41-8$ & 192 & $\mathrm{ng} / \mathrm{L}$ & $\operatorname{irl}$ \\
\hline Norverapamil & 68007 & LCM56 & $67018-85-3$ & 8.5 & $\mathrm{ng} / \mathrm{L}$ & $\operatorname{irl}$ \\
\hline Oxycodone & 67495 & LCM56 & $76-42-6$ & 24 & $\mathrm{ng} / \mathrm{L}$ & irl \\
\hline Oxycodone-d3 (surrogate) & 91778 & LCM56 & - & N/A & pct & $\mathrm{mrl}$ \\
\hline Paroxetine & 67527 & LCM56 & 61869-08-7 & 20 & $\mathrm{ng} / \mathrm{L}$ & $\operatorname{irl}$ \\
\hline Penciclovir & 68021 & LCM56 & $39809-25-1$ & 40 & $\mathrm{ng} / \mathrm{L}$ & irl \\
\hline Pentoxifylline & 67480 & LCM56 & $6493-05-6$ & 9.3 & $\mathrm{ng} / \mathrm{L}$ & $\operatorname{irl}$ \\
\hline Phenazopyridine & 68008 & LCM56 & $94-78-0$ & 13 & $\mathrm{ng} / \mathrm{L}$ & $\operatorname{irl}$ \\
\hline Phendimetrazine & 67496 & LCM56 & $634-03-7$ & 31 & $\mathrm{ng} / \mathrm{L}$ & $\operatorname{irl}$ \\
\hline Phenytoin & 67466 & LCM56 & $57-41-0$ & 188 & $\mathrm{ng} / \mathrm{L}$ & $\operatorname{irl}$ \\
\hline Piperonyl butoxide & 67435 & LCM56 & $51-03-6$ & 3 & $\mathrm{ng} / \mathrm{L}$ & irl \\
\hline Pseudoephedrine-d3 surrogate (surrogate) & 91787 & LCM56 & - & N/A & pct & $\mathrm{mrl}$ \\
\hline Quinine & 68011 & LCM56 & $130-95-0$ & 79 & $\mathrm{ng} / \mathrm{L}$ & $\operatorname{irl}$ \\
\hline Ractopamine & 52814 & LCM56 & $97825-25-7$ & 20 & $\mathrm{ng} / \mathrm{L}$ & RLDQC \\
\hline Raloxifene & 67530 & LCM56 & $84449-90-1$ & 9.7 & $\mathrm{ng} / \mathrm{L}$ & irl \\
\hline Ranitidine & 67452 & LCM56 & $66357-35-5$ & 192 & $\mathrm{ng} / \mathrm{L}$ & irl \\
\hline Sertraline & 67528 & LCM56 & $79617-96-2$ & 16 & $\mathrm{ng} / \mathrm{L}$ & $\operatorname{irl}$ \\
\hline Sitagliptin & 67531 & LCM56 & $790712-60-6$ & 97 & $\mathrm{ng} / \mathrm{L}$ & $\operatorname{irl}$ \\
\hline Sulfadimethoxine & 67503 & LCM56 & $122-11-2$ & 65 & $\mathrm{ng} / \mathrm{L}$ & irl \\
\hline Sulfamethizole & 67476 & LCM56 & $144-82-1$ & 104 & $\mathrm{ng} / \mathrm{L}$ & irl \\
\hline Sulfamethoxazole & 67454 & LCM56 & $723-46-6$ & 26 & $\mathrm{ng} / \mathrm{L}$ & $\operatorname{irl}$ \\
\hline Sulfamethoxazole-(phenyl-13C6) (surrogate) & 91782 & LCM56 & - & N/A & pct & $\mathrm{mrl}$ \\
\hline Tamoxifen & 68015 & LCM56 & $10540-29-1$ & 80 & $\mathrm{ng} / \mathrm{L}$ & $\mathrm{mrl}$ \\
\hline
\end{tabular}


Table 2.4. U.S. Geological Survey National Water Quality Laboratory Schedule 2440 for pharmaceutical compounds in water.Continued

[Methods are described in Furlong and others (2014). Parameter names are as shown in the U.S. Geological Survey (USGS) National Water Information System (NWIS) database and do not include special characters or formatting. Abbreviations: CAS, Chemical Abstracts Service1; irl, interim reporting level; mrl, minimum reporting level; N/A, not applicable; ng/L, nanogram per liter; NWQL, USGS National Water Quality Laboratory; pct, percent; -, no data]

\begin{tabular}{|c|c|c|c|c|c|c|}
\hline Parameter name & $\begin{array}{c}\text { NWIS } \\
\text { parameter } \\
\text { code }\end{array}$ & $\begin{array}{c}\text { NWIS } \\
\text { method } \\
\text { code }\end{array}$ & CAS number & $\begin{array}{c}\text { Reporting } \\
\text { level }\end{array}$ & Unit & $\begin{array}{l}\text { Reporting } \\
\text { level type }\end{array}$ \\
\hline Temazepam & 67471 & LCM56 & $846-50-4$ & 18 & $\mathrm{ng} / \mathrm{L}$ & irl \\
\hline Temazepam-d5 (surrogate) & 91780 & LCM56 & - & $\mathrm{N} / \mathrm{A}$ & pet & $\mathrm{mrl}$ \\
\hline Theophylline & 67494 & LCM56 & $58-55-9$ & 41 & $\mathrm{ng} / \mathrm{L}$ & irl \\
\hline Tiotropium & 67508 & LCM56 & $186691-13-4$ & 43 & $\mathrm{ng} / \mathrm{L}$ & irl \\
\hline Tramadol & 67517 & LCM56 & $27203-92-5$ & 15 & $\mathrm{ng} / \mathrm{L}$ & irl \\
\hline Triamterene & 67475 & LCM56 & $396-01-0$ & 5.2 & $\mathrm{ng} / \mathrm{L}$ & irl \\
\hline Trimethoprim & 67456 & LCM56 & $738-70-5$ & 19 & $\mathrm{ng} / \mathrm{L}$ & irl \\
\hline Verapamil & 67472 & LCM56 & $52-53-9$ & 15 & $\mathrm{ng} / \mathrm{L}$ & irl \\
\hline Warfarin & 67457 & LCM56 & $81-81-2$ & 6 & $\mathrm{ng} / \mathrm{L}$ & Irl \\
\hline
\end{tabular}

1This report contains CAS Registry Numbers ${ }^{\circledR}$, which is a Registered Trademark of the American Chemical Society. CAS recommends the verification of the CAS Registry Numbers through CAS Client Services ${ }^{\text {SM }}$. 
Table 2.5. U.S. Geological Survey National Water Quality Laboratory Schedule 4433 for organic wastewater indicator compounds in water.

[Methods are described in Zaugg and others (2006). Parameter names are as shown in the U.S. Geological Survey (USGS) National Water Information System (NWIS) database and do not include special characters or formatting. Abbreviations: CAS, Chemical Abstracts Service'; irl, interim reporting level; mL, milliliter; mrl, minimum reporting level; N/A, not applicable; NWQL, USGS National Water Quality Laboratory; pct, percent; —, no data; $\mu \mathrm{g} / \mathrm{L}$, microgram per liter]

\begin{tabular}{|c|c|c|c|c|c|c|}
\hline Parameter name & $\begin{array}{c}\text { NWIS } \\
\text { parameter } \\
\text { code }\end{array}$ & $\begin{array}{c}\text { NWIS } \\
\text { method } \\
\text { code }\end{array}$ & CAS number & $\begin{array}{c}\text { Reporting } \\
\text { level }\end{array}$ & Unit & $\begin{array}{l}\text { Reporting } \\
\text { level type }\end{array}$ \\
\hline 1,4-Dichlorobenzene & 34571 & GCM99 & $106-46-7$ & 0.08 & $\mu \mathrm{g} / \mathrm{L}$ & irl \\
\hline 1-Methylnaphthalene & 81696 & GCM99 & $90-12-0$ & 0.04 & $\mu \mathrm{g} / \mathrm{L}$ & irl \\
\hline 2,2',4,4'-Tetrabromodiphenylether (PBDE 47) & 63147 & GCM99 & $5436-43-1$ & 0.04 & $\mu \mathrm{g} / \mathrm{L}$ & irl \\
\hline 2,6-Dimethylnaphthalene & 62805 & GCM99 & $581-42-0$ & 0.04 & $\mu \mathrm{g} / \mathrm{L}$ & irl \\
\hline 3,4-Dichlorophenyl isocyanate & 63145 & GCM99 & $102-36-3$ & 0.32 & $\mu \mathrm{g} / \mathrm{L}$ & $\operatorname{irl}$ \\
\hline 3-beta-Coprostanol & 62806 & GCM99 & $360-68-9$ & 1.6 & $\mu \mathrm{g} / \mathrm{L}$ & irl \\
\hline 3-Methyl-1(H)-indole (Skatole) & 62807 & GCM99 & $83-34-1$ & 0.04 & $\mu \mathrm{g} / \mathrm{L}$ & irl \\
\hline 3-tert-Butyl-4-hydroxy anisole (BHA) & 61702 & GCM99 & $121-00-6$ & 0.16 & $\mu \mathrm{g} / \mathrm{L}$ & irl \\
\hline 4-Nonylphenol monoethoxylate (NP1EO), all isomers & 61704 & GCM99 & $104-35-8$ & 1.6 & $\mu \mathrm{g} / \mathrm{L}$ & irl \\
\hline 4-tert-Octylphenol & 62810 & GCM99 & $140-66-9$ & 0.4 & $\mu \mathrm{g} / \mathrm{L}$ & irl \\
\hline 4-tert-Octylphenol diethoxylate, aka OP2EO & 62486 & GCM99 & 2315-61-9 & 0.2 & $\mu \mathrm{g} / \mathrm{L}$ & irl \\
\hline 4-tert-Octylphenol monoethoxylate, aka OP1EO & 62485 & GCM99 & $2315-67-5$ & 0.6 & $\mu \mathrm{g} / \mathrm{L}$ & irl \\
\hline 5-Methyl-1H-benzotriazole & 61944 & GCM99 & $136-85-6$ & 0.32 & $\mu \mathrm{g} / \mathrm{L}$ & $\operatorname{irl}$ \\
\hline Acetophenone & 62811 & GCM99 & $98-86-2$ & 0.4 & $\mu \mathrm{g} / \mathrm{L}$ & irl \\
\hline Acetyl hexamethyl tetrahydronaphthalene (AHTN) & 62812 & GCM99 & $21145-77-7$ & 0.04 & $\mu \mathrm{g} / \mathrm{L}$ & irl \\
\hline Anthracene & 34220 & GCM99 & $120-12-7$ & 0.02 & $\mu \mathrm{g} / \mathrm{L}$ & irl \\
\hline Anthraquinone & 62813 & GCM99 & $84-65-1$ & 0.04 & $\mu \mathrm{g} / \mathrm{L}$ & irl \\
\hline Bisphenol A & 62816 & GCM99 & $80-05-7$ & 0.04 & $\mu \mathrm{g} / \mathrm{L}$ & irl \\
\hline Bisphenol A-d3 (surrogate) & 62839 & GCM99 & - & N/A & pct & $\mathrm{mrl}$ \\
\hline Bromacil & 30234 & GCM99 & $314-40-9$ & 0.16 & $\mu \mathrm{g} / \mathrm{L}$ & irl \\
\hline Bromoform & 32104 & GCM99 & $75-25-2$ & 0.16 & $\mu \mathrm{g} / \mathrm{L}$ & irl \\
\hline Caffeine & 81436 & GCM99 & $58-08-2$ & 0.08 & $\mu \mathrm{g} / \mathrm{L}$ & irl \\
\hline Caffeine-C13 (surrogate) & 62840 & GCM99 & - & N/A & pet & $\mathrm{mrl}$ \\
\hline Camphor & 62817 & GCM99 & $76-22-2$ & 0.08 & $\mu \mathrm{g} / \mathrm{L}$ & irl \\
\hline Carbaryl & 39750 & GCM99 & $63-25-2$ & 0.06 & $\mu \mathrm{g} / \mathrm{L}$ & irl \\
\hline Carbazole & 77571 & GCM99 & $86-74-8$ & 0.02 & $\mu \mathrm{g} / \mathrm{L}$ & irl \\
\hline Chlorpyrifos & 38932 & GCM99 & $2921-88-2$ & 0.12 & $\mu \mathrm{g} / \mathrm{L}$ & irl \\
\hline Cholesterol & 62818 & GCM99 & $57-88-5$ & 1.6 & $\mu \mathrm{g} / \mathrm{L}$ & irl \\
\hline Cotinine & 61945 & GCM99 & $486-56-6$ & 0.08 & $\mu \mathrm{g} / \mathrm{L}$ & irl \\
\hline
\end{tabular}


Table 2.5. U.S. Geological Survey National Water Quality Laboratory Schedule 4433 for organic wastewater indicator compounds in water-Continued

[Methods are described in Zaugg and others (2006). Parameter names are as shown in the U.S. Geological Survey (USGS) National Water Information System (NWIS) database and do not include special characters or formatting. Abbreviations: CAS, Chemical Abstracts Service1; irl, interim reporting level; mL, milliliter; mrl, minimum reporting level; N/A, not applicable; NWQL, USGS National Water Quality Laboratory; pct, percent; -, no data; $\mu \mathrm{g} / \mathrm{L}$, microgram per liter]

\begin{tabular}{|c|c|c|c|c|c|c|}
\hline Parameter name & $\begin{array}{c}\text { NWIS } \\
\text { parameter } \\
\text { code }\end{array}$ & $\begin{array}{c}\text { NWIS } \\
\text { method } \\
\text { code }\end{array}$ & CAS number & $\begin{array}{l}\text { Reporting } \\
\text { level }\end{array}$ & Unit & $\begin{array}{l}\text { Reporting } \\
\text { level type }\end{array}$ \\
\hline d-Limonene & 62819 & GCM99 & $5989-27-5$ & 0.16 & $\mu \mathrm{g} / \mathrm{L}$ & $\operatorname{irl}$ \\
\hline Decafluorobiphenyl (surrogate) & 62841 & GCM99 & $434-90-2$ & $\mathrm{~N} / \mathrm{A}$ & pct & $\mathrm{mrl}$ \\
\hline Diazinon & 39570 & GCM99 & $333-41-5$ & 0.32 & $\mu \mathrm{g} / \mathrm{L}$ & $\operatorname{irl}$ \\
\hline Dichlorvos & 30218 & GCM99 & $62-73-7$ & 0.08 & $\mu \mathrm{g} / \mathrm{L}$ & $\operatorname{irl}$ \\
\hline Diethyl phthalate & 34336 & GCM99 & $84-66-2$ & 0.4 & $\mu \mathrm{g} / \mathrm{L}$ & $\operatorname{irl}$ \\
\hline Fluoranthene & 34376 & GCM99 & $206-44-0$ & 0.02 & $\mu \mathrm{g} / \mathrm{L}$ & $\operatorname{irl}$ \\
\hline Fluoranthene-d10 (surrogate) & 62842 & GCM99 & 93951-69-0 & N/A & pet & $\mathrm{mrl}$ \\
\hline Hexahydrohexamethylcyclopentabenzopyran (HHCB) & 62823 & GCM99 & $1222-05-5$ & 0.04 & $\mu \mathrm{g} / \mathrm{L}$ & $\operatorname{irl}$ \\
\hline Indole & 62824 & GCM99 & $120-72-9$ & 0.04 & $\mu \mathrm{g} / \mathrm{L}$ & $\operatorname{irl}$ \\
\hline Isoborneol & 62825 & GCM99 & $124-76-5$ & 0.09 & $\mu \mathrm{g} / \mathrm{L}$ & $\operatorname{irl}$ \\
\hline Isophorone & 34408 & GCM99 & $78-59-1$ & 0.05 & $\mu \mathrm{g} / \mathrm{L}$ & irl \\
\hline Isopropylbenzene & 77223 & GCM99 & $98-82-8$ & 0.04 & $\mu \mathrm{g} / \mathrm{L}$ & irl \\
\hline Isoquinoline & 62826 & GCM99 & $119-65-3$ & 0.8 & $\mu \mathrm{g} / \mathrm{L}$ & $\operatorname{irl}$ \\
\hline Menthol & 62827 & GCM99 & $89-78-1$ & 0.32 & $\mu \mathrm{g} / \mathrm{L}$ & $\operatorname{irl}$ \\
\hline Metalaxyl & 04254 & GCM99 & $57837-19-1$ & 0.16 & $\mu \mathrm{g} / \mathrm{L}$ & $\operatorname{irl}$ \\
\hline Methyl salicylate & 62828 & GCM99 & $119-36-8$ & 0.08 & $\mu \mathrm{g} / \mathrm{L}$ & irl \\
\hline Metolachlor & 82612 & GCM99 & $51218-45-2$ & 0.04 & $\mu \mathrm{g} / \mathrm{L}$ & $\operatorname{irl}$ \\
\hline N,N-Diethyl-meta-toluamide (DEET) & 61947 & GCM99 & $134-62-3$ & 0.04 & $\mu \mathrm{g} / \mathrm{L}$ & $\operatorname{irl}$ \\
\hline Naphthalene & 34696 & GCM99 & $91-20-3$ & 0.02 & $\mu \mathrm{g} / \mathrm{L}$ & irl \\
\hline $\mathrm{p}$-Cresol & 77146 & GCM99 & $106-44-5$ & 0.08 & $\mu \mathrm{g} / \mathrm{L}$ & irl \\
\hline para-Nonylphenol (total) (branched) & 62829 & GCM99 & $84852-15-3$ & 1.6 & $\mu \mathrm{g} / \mathrm{L}$ & irl \\
\hline Pentachlorophenol & 39032 & GCM99 & $87-86-5$ & 1.6 & $\mu \mathrm{g} / \mathrm{L}$ & $\mathrm{irl}$ \\
\hline Phenanthrene & 34461 & GCM99 & $85-01-8$ & 0.02 & $\mu \mathrm{g} / \mathrm{L}$ & $\mathrm{irl}$ \\
\hline Phenol & 34694 & GCM99 & $108-95-2$ & 0.16 & $\mu \mathrm{g} / \mathrm{L}$ & irl \\
\hline Prometon & 39056 & GCM99 & $1610-18-0$ & 0.16 & $\mu \mathrm{g} / \mathrm{L}$ & $\operatorname{irl}$ \\
\hline Pyrene & 34469 & GCM99 & $129-00-0$ & 0.02 & $\mu \mathrm{g} / \mathrm{L}$ & $\operatorname{irl}$ \\
\hline Sample volume & 99963 & GCM99 & - & $\mathrm{N} / \mathrm{A}$ & $\mathrm{mL}$ & $\operatorname{irl}$ \\
\hline Tetrachloroethylene & 34475 & GCM99 & $127-18-4$ & 0.16 & $\mu \mathrm{g} / \mathrm{L}$ & $\operatorname{irl}$ \\
\hline Tributyl phosphate & 62832 & GCM99 & $126-73-8$ & 0.064 & $\mu \mathrm{g} / \mathrm{L}$ & irl \\
\hline Triclosan & 61708 & GCM99 & $3380-34-5$ & 0.32 & $\mu \mathrm{g} / \mathrm{L}$ & $\operatorname{irl}$ \\
\hline Triethyl citrate (ethyl citrate) & 62833 & GCM99 & $77-93-0$ & 0.04 & $\mu \mathrm{g} / \mathrm{L}$ & irl \\
\hline Triphenyl phosphate & 62834 & GCM99 & $115-86-6$ & 0.08 & $\mu \mathrm{g} / \mathrm{L}$ & $\operatorname{irl}$ \\
\hline Tris(2-butoxyethyl)phosphate & 62830 & GCM99 & $78-51-3$ & 0.64 & $\mu \mathrm{g} / \mathrm{L}$ & $\operatorname{irl}$ \\
\hline Tris(2-chloroethyl)phosphate & 62831 & GCM99 & $115-96-8$ & 0.16 & $\mu \mathrm{g} / \mathrm{L}$ & irl \\
\hline Tris(dichloroisopropyl)phosphate & 61707 & GCM99 & $13674-87-8$ & 0.32 & $\mu \mathrm{g} / \mathrm{L}$ & $\operatorname{Irl}$ \\
\hline
\end{tabular}

1This report contains CAS Registry Numbers ${ }^{\circledR}$, which is a Registered Trademark of the American Chemical Society. CAS recommends the verification of the CAS Registry Numbers through CAS Client Services $\mathrm{SM}$. 
Table 2.6. U.S. Geological Survey National Water Quality Laboratory Schedule 5433 for organic wastewater indicator compounds in bed sediment.

[Methods are decribed in Burkhardt and others (2006). Parameter names are as shown in the U.S. Geological Survey (USGS) National Water Information System (NWIS) database and do not include special characters or formatting. Abbreviations: NWQL, USGS National Water Quality Laboratory;

CAS, Chemical Abstracts Servicel; N/A, not applicable; $\mu \mathrm{g} / \mathrm{kg}$, microgram per kilogram; pct, percent; g, gram; irl, interim reporting level; —, no data]

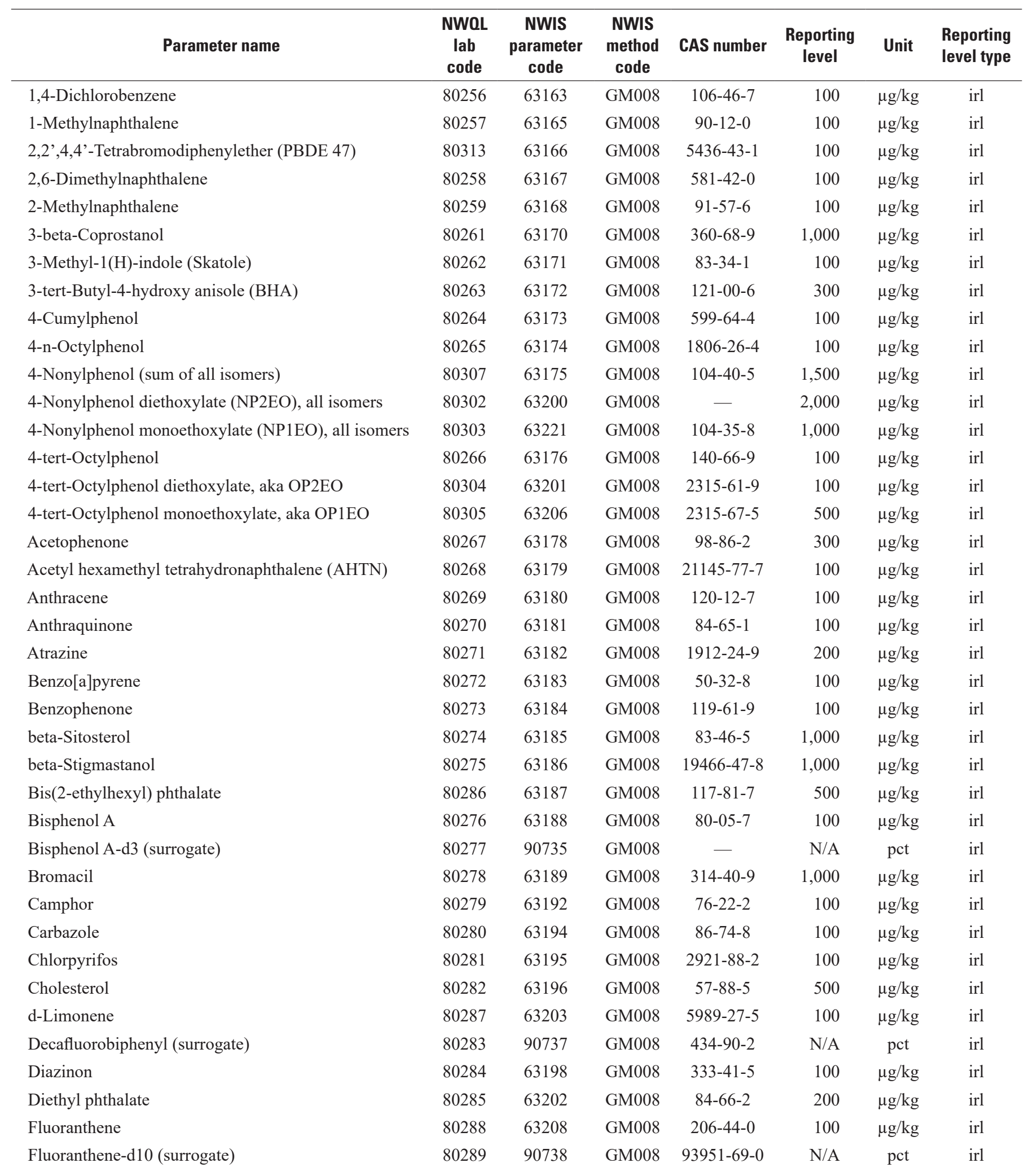


Table 2.6. U.S. Geological Survey National Water Quality Laboratory Schedule 5433 for organic wastewater indicator compounds in bed sediment.-Continued

[Methods are decribed in Burkhardt and others (2006). Parameter names are as shown in the U.S. Geological Survey (USGS) National Water Information System (NWIS) database and do not include special characters or formatting. Abbreviations: NWQL, USGS National Water Quality Laboratory;

CAS, Chemical Abstracts Service ${ }^{1}$; N/A, not applicable; $\mu \mathrm{g} / \mathrm{kg}$, microgram per kilogram; pet, percent; g, gram; irl, interim reporting level; —, no data]

\begin{tabular}{|c|c|c|c|c|c|c|c|}
\hline Parameter name & $\begin{array}{c}\text { NWQL } \\
\text { lab } \\
\text { code }\end{array}$ & $\begin{array}{c}\text { NWIS } \\
\text { parameter } \\
\text { code }\end{array}$ & $\begin{array}{l}\text { NWIS } \\
\text { method } \\
\text { code }\end{array}$ & CAS number & $\begin{array}{c}\text { Reporting } \\
\text { level }\end{array}$ & Unit & $\begin{array}{l}\text { Reporting } \\
\text { level type }\end{array}$ \\
\hline Hexahydrohexamethylcyclopentabenzopyran (HHCB) & 80290 & 63209 & GM008 & $1222-05-5$ & 100 & $\mu \mathrm{g} / \mathrm{kg}$ & irl \\
\hline Indole & 80291 & 63210 & GM008 & $120-72-9$ & 200 & $\mu \mathrm{g} / \mathrm{kg}$ & irl \\
\hline Isoborneol & 80292 & 63211 & GM008 & $124-76-5$ & 100 & $\mu \mathrm{g} / \mathrm{kg}$ & irl \\
\hline Isophorone & 80293 & 63212 & GM008 & $78-59-1$ & 100 & $\mu \mathrm{g} / \mathrm{kg}$ & $\operatorname{irl}$ \\
\hline Isoquinoline & 80295 & 63214 & GM008 & $119-65-3$ & 200 & $\mu \mathrm{g} / \mathrm{kg}$ & irl \\
\hline Menthol & 80296 & 63215 & GM008 & $89-78-1$ & 100 & $\mu \mathrm{g} / \mathrm{kg}$ & irl \\
\hline Metolachlor & 80299 & 63218 & GM008 & $51218-45-2$ & 100 & $\mu \mathrm{g} / \mathrm{kg}$ & irl \\
\hline N,N-Diethyl-meta-toluamide (DEET) & 80300 & 63219 & GM008 & $134-62-3$ & 200 & $\mu \mathrm{g} / \mathrm{kg}$ & irl \\
\hline Phenol & 80310 & 63225 & GM008 & $108-95-2$ & 100 & $\mu \mathrm{g} / \mathrm{kg}$ & irl \\
\hline Prometon & 80311 & 63226 & GM008 & $1610-18-0$ & 100 & $\mu \mathrm{g} / \mathrm{kg}$ & irl \\
\hline Pyrene & 80312 & 63227 & GM008 & $129-00-0$ & 100 & $\mu \mathrm{g} / \mathrm{kg}$ & irl \\
\hline Sample weight, grams & 80320 & 91100 & GM008 & - & $\mathrm{N} / \mathrm{A}$ & $\mathrm{g}$ & irl \\
\hline Tributyl phosphate & 80317 & 63231 & GM008 & $126-73-8$ & 100 & $\mu \mathrm{g} / \mathrm{kg}$ & irl \\
\hline Triclosan & 80318 & 63232 & GM008 & $3380-34-5$ & 100 & $\mu \mathrm{g} / \mathrm{kg}$ & irl \\
\hline Triphenyl phosphate & 80319 & 63234 & GM008 & $115-86-6$ & 100 & $\mu \mathrm{g} / \mathrm{kg}$ & irl \\
\hline Tris(2-butoxyethyl)phosphate & 80314 & 63229 & GM008 & $78-51-3$ & 300 & $\mu \mathrm{g} / \mathrm{kg}$ & irl \\
\hline Tris(2-chloroethyl)phosphate & 80315 & 63230 & GM008 & $115-96-8$ & 200 & $\mu \mathrm{g} / \mathrm{kg}$ & irl \\
\hline
\end{tabular}

${ }^{1}$ This report contains CAS Registry Numbers ${ }^{\circledR}$, which is a Registered Trademark of the American Chemical Society. CAS recommends the verification of the CAS Registry Numbers through CAS Client ServicesSM. 
Table 2.7. U.S. Environmental Protection Agency Laboratory Schedule for polycyclic aromatic hydrocarbons in bed sediment, using U.S. Environmental Protection Agency method 8270D.

[Methods are described in U.S. Environmental Protection Agency (2014). Abbreviations: $\mu$ g/kg, microgram per kilogram; pct, percent; N/A, not applicable]

\begin{tabular}{lcc}
\hline \multicolumn{1}{c}{ Parameter name } & Reporting level & Unit \\
\hline Acenaphthylene, bed sediment, recoverable, dry weight & 25 & $\mu \mathrm{g} / \mathrm{kg}$ \\
Acenaphthene, bed sediment, recoverable, dry weight & 25 & $\mu \mathrm{g} / \mathrm{kg}$ \\
Anthracene, bed sediment, recoverable, dry weight & 25 & $\mu \mathrm{g} / \mathrm{kg}$ \\
Benzo[b]fluoranthene, bed sediment, recoverable & $\mu \mathrm{g} / \mathrm{kg}$ \\
Benzo[k]fluoranthene, bed sediment, recoverable & 25 & $\mu \mathrm{g} / \mathrm{kg}$ \\
Benzo[a]pyrene, bed sediment, recoverable, dry weight & 25 & $\mu \mathrm{g} / \mathrm{kg}$ \\
Chrysene, bed sediment, recoverable, dry weight & 25 & $\mu \mathrm{g} / \mathrm{kg}$ \\
Fluoranthene, bed sediment, recoverable, dry weight & 25 & $\mu \mathrm{g} / \mathrm{kg}$ \\
9H-Fluorene, bed sediment, recoverable, dry weight & 25 & $\mu \mathrm{g} / \mathrm{kg}$ \\
Indeno[1,2,3-cd]pyrene, bed sediment, recoverable, dry weight & $25 \mathrm{~g} / \mathrm{kg}$ \\
Naphthalene, bed sediment, recoverable, dry weight & 25 & $\mu \mathrm{g} / \mathrm{kg}$ \\
Phenanthrene, bed sediment, recoverable, dry weight & 25 & $\mu \mathrm{g} / \mathrm{kg}$ \\
Pyrene, bed sediment, recoverable, dry weight & 25 & $\mu \mathrm{k} / \mathrm{kg}$ \\
Benzo[ghi]perylene, bed sediment, recoverable, dry weight & 25 & $\mu \mathrm{g} / \mathrm{kg}$ \\
Benzo[a]anthracene, bed sediment, recoverable, dry weight & 25 & $\mu \mathrm{g} / \mathrm{kg}$ \\
Dibenzo[a,h]anthracene, bed sediment, recoverable, dry weight & 25 & $\mu \mathrm{g} / \mathrm{kg}$ \\
2-Methylnaphthalene, bed sediment, recoverable, dry weight & 25 & $\mathrm{gg} / \mathrm{kg}$ \\
Moisture content, bed sediment, dry weight & 25 & $\mathrm{pct}$ \\
\hline
\end{tabular}


Table 2.8. U.S. Geological Survey National Water Quality Laboratory custom method 8093 for halogenated organic compounds in bed sediment.

[Methods are described in Mahler and others (2009), and Wagner and others (2014). Parameter names are as shown in the U.S. Geological Survey (USGS) National Water Information System (NWIS) database and do not include special characters or formatting. Abbreviations: NWQL, USGS National Water Quality Laboratory; CAS, Chemical Abstracts Service 1 ; N/A, not applicable; $\mu \mathrm{g} / \mathrm{kg}$, microgram per kilogram; pct, percent; g, gram; irl, interim reporting level; -, no data]

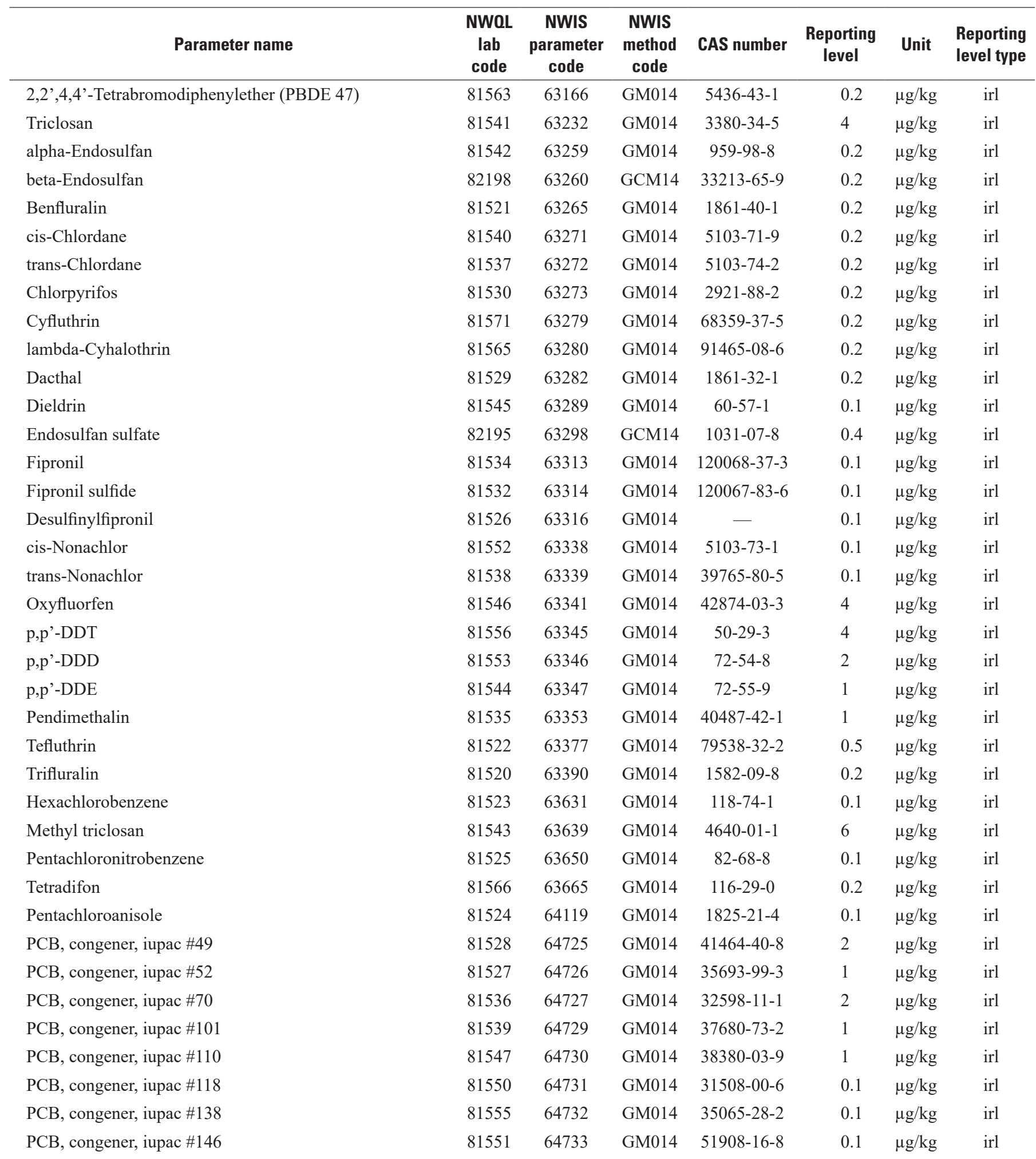


Table 2.8. U.S. Geological Survey National Water Quality Laboratory custom method 8093 for halogenated organic compounds in bed sediment.-Continued

[Methods are described in Mahler and others (2009), and Wagner and others (2014). Parameter names are as shown in the U.S. Geological Survey (USGS) National Water Information System (NWIS) database and do not include special characters or formatting. Abbreviations: NWQL, USGS National Water Quality Laboratory; CAS, Chemical Abstracts Service ${ }^{1}$; N/A, not applicable; $\mu \mathrm{g} / \mathrm{kg}$, microgram per kilogram; pct, percent; g, gram; irl, interim reporting level; -, no data]

\begin{tabular}{|c|c|c|c|c|c|c|c|}
\hline Parameter name & $\begin{array}{c}\text { NWOL } \\
\text { lab } \\
\text { code }\end{array}$ & $\begin{array}{c}\text { NWIS } \\
\text { parameter } \\
\text { code }\end{array}$ & $\begin{array}{c}\text { NWIS } \\
\text { method } \\
\text { code }\end{array}$ & CAS number & $\begin{array}{c}\text { Reporting } \\
\text { level }\end{array}$ & Unit & $\begin{array}{l}\text { Reporting } \\
\text { level type }\end{array}$ \\
\hline PCB, congener, iupac \#149 & 81549 & 64734 & GM014 & $38380-04-0$ & 1 & $\mu \mathrm{g} / \mathrm{kg}$ & irl \\
\hline PCB, congener, iupac \#151 & 81548 & 64735 & GM014 & $52663-63-5$ & 0.1 & $\mu \mathrm{g} / \mathrm{kg}$ & irl \\
\hline PCB, congener, iupac \#170 & 81564 & 64736 & GM014 & $35065-30-6$ & 0.1 & $\mu \mathrm{g} / \mathrm{kg}$ & $\operatorname{irl}$ \\
\hline PCB, congener, iupac \#180 & 81561 & 64739 & GM014 & $35065-29-3$ & 0.1 & $\mu \mathrm{g} / \mathrm{kg}$ & irl \\
\hline PCB, congener, iupac \#183 & 81558 & 64740 & GM014 & $52663-69-1$ & 0.1 & $\mu \mathrm{g} / \mathrm{kg}$ & irl \\
\hline PCB, congener, iupac \#187 & 81557 & 64741 & GM014 & $52663-68-0$ & 0.1 & $\mu \mathrm{g} / \mathrm{kg}$ & $\operatorname{irl}$ \\
\hline PCB, congener, iupac \#194 & 81568 & 64742 & GM014 & $35694-08-7$ & 0.1 & $\mu \mathrm{g} / \mathrm{kg}$ & irl \\
\hline 2,2',3,4,4'-Pentabromodiphenyl ether (PBDE 85) & 81573 & 64854 & GM014 & $182346-21-0$ & 0.1 & $\mu \mathrm{g} / \mathrm{kg}$ & $\operatorname{irl}$ \\
\hline 2,2',4,4',5-Pentabromodiphenyl ether (PBDE 99) & 81572 & 64855 & GM014 & $60348-60-9$ & 0.2 & $\mu \mathrm{g} / \mathrm{kg}$ & irl \\
\hline 2,2',4,4',6-Pentabromodiphenyl ether (PBDE 100) & 81569 & 64856 & GM014 & $189084-64-8$ & 0.1 & $\mu \mathrm{g} / \mathrm{kg}$ & irl \\
\hline 2,2',3,4,4',5'-Hexabromodiphenyl ether (PBDE138) & 81576 & 64857 & GM014 & $182677-30-1$ & 0.1 & $\mu \mathrm{g} / \mathrm{kg}$ & irl \\
\hline 2,2',4,4',5,5'-Hexabromodiphenylether (PBDE 153) & 81575 & 64858 & GM014 & $68631-49-2$ & 0.1 & $\mu \mathrm{g} / \mathrm{kg}$ & irl \\
\hline 2,2',4,4',5,6'-Hexabromodiphenyl ether (PBDE 154) & 81574 & 64859 & GM014 & $207122-15-4$ & 0.1 & $\mu \mathrm{g} / \mathrm{kg}$ & $\operatorname{irl}$ \\
\hline 2,2',3,4,4',5',6-Heptabromodiphenyl ether (PBDE 183) & 81577 & 64860 & GM014 & $207122-16-5$ & 0.1 & $\mu \mathrm{g} / \mathrm{kg}$ & $\operatorname{irl}$ \\
\hline Oxychlordane & 81533 & 64866 & GM014 & $27304-13-8$ & 1 & $\mu \mathrm{g} / \mathrm{kg}$ & $\operatorname{irl}$ \\
\hline Pentabromotoluene & 81554 & 64867 & GM014 & $87-83-2$ & 1 & $\mu \mathrm{g} / \mathrm{kg}$ & irl \\
\hline 4,4'-Dibromooctafluorobiphenyl (surrogate) & 81579 & 91785 & GM014 & $10386-84-2$ & $\mathrm{~N} / \mathrm{A}$ & pct & N/A \\
\hline p,p'-DDT-d8 (surrogate) & 81580 & 91828 & GM014 & - & N/A & pct & N/A \\
\hline
\end{tabular}

${ }^{1}$ This report contains CAS Registry Numbers ${ }^{\circledR}$, which is a Registered Trademark of the American Chemical Society. CAS recommends the verification of the CAS Registry Numbers through CAS Client Services ${ }^{\mathrm{SM}}$. 
Table 2.9. U.S. Geological Survey National Water Quality Laboratory Schedule 6434 for hormone compounds in bed sediment.

[Custom method derived from Foreman and others (2012). Parameter names are as shown in the U.S. Geological Survey (USGS) National Water Information System (NWIS) database and do not include special characters or formatting. Abbreviations: CAS, Chemical Abstracts Service1; g, gram; N/A, not applicable; irl, interim reporting level; MRL, minimum reporting level; pct, percent; $\mu \mathrm{g} / \mathrm{kg}$, microgram per kilogram; —, no data]

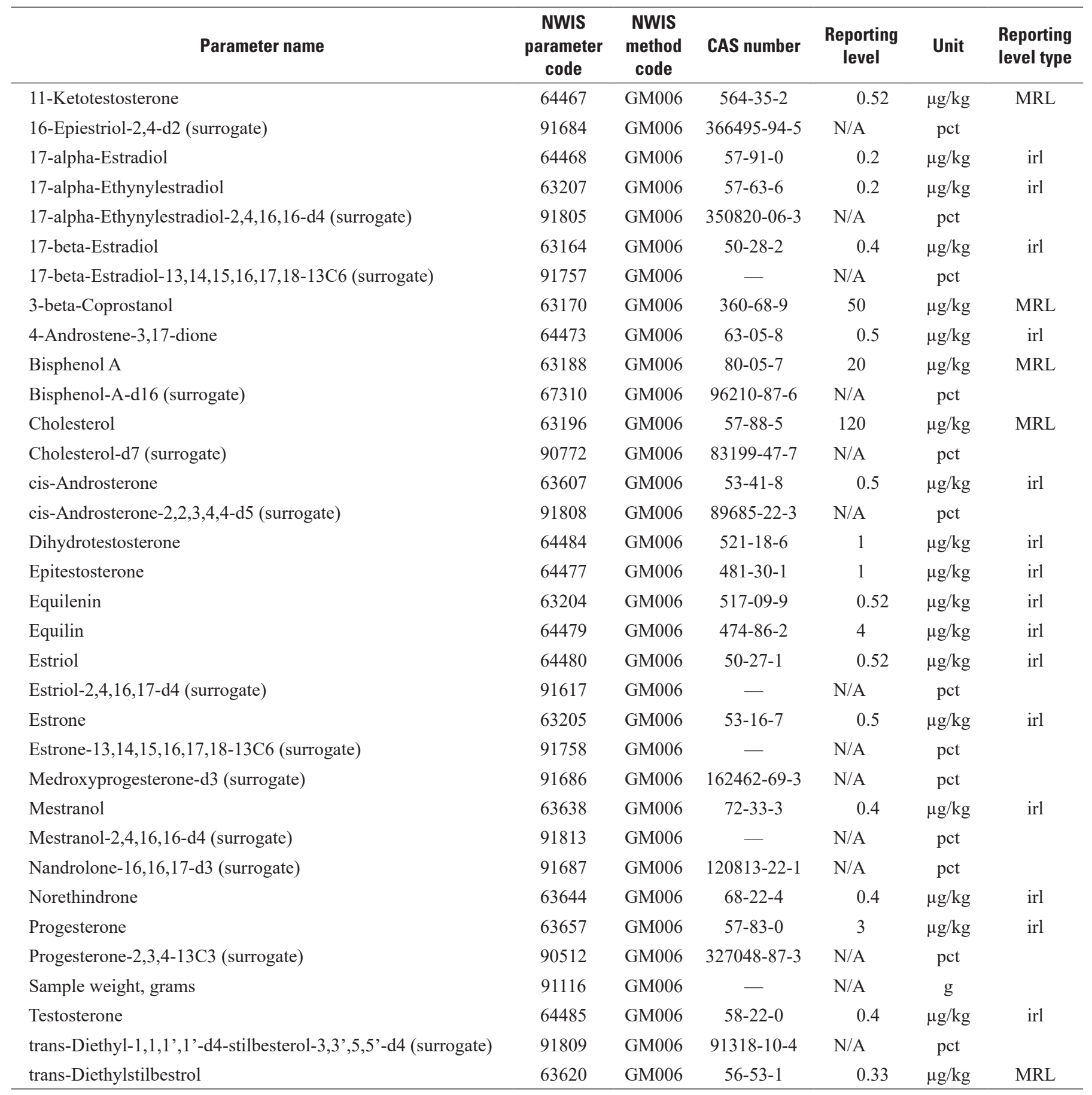

${ }^{1}$ This report contains CAS Registry Numbers ${ }^{\circledR}$, which is a Registered Trademark of the American Chemical Society. CAS recommends the verification of the CAS Registry Numbers through CAS Client ServicesSM. 
Table 2.10. U.S. Geological Survey National Water Quality Laboratory Schedule 1632 for chlorophyll a, pheophytin a, and ash-free dry mass in periphyton.

[NWQL, U.S. Geological Survey (USGS) National Water Quality Laboratory; NWIS, USGS National Water Information System; CAS, Chemical Abstracts Service ${ }^{1} ; \mathrm{g} / \mathrm{m}^{2}$, gram per square meter; $\mathrm{mg} / \mathrm{m}^{2}$, milligram per square meter; mrl, minimum reporting level; lrl, laboratory reporting level; EPA, U.S. Environmental Protection Agency; —, no data]

\begin{tabular}{|c|c|c|c|c|c|c|c|c|c|}
\hline Parameter name & $\begin{array}{c}\text { NWQL } \\
\text { lab } \\
\text { code }\end{array}$ & $\begin{array}{c}\text { NWIS } \\
\text { parameter } \\
\text { code }\end{array}$ & $\begin{array}{l}\text { NWIS } \\
\text { method } \\
\text { code }\end{array}$ & $\begin{array}{c}\text { CAS } \\
\text { number }\end{array}$ & $\begin{array}{l}\text { Reporting } \\
\text { level }\end{array}$ & Unit & $\begin{array}{l}\text { Reporting } \\
\text { level type }\end{array}$ & Method citation & Method \\
\hline Chlorophyll $a$ & 3153 & 70957 & 00050 & $479-61-8$ & 0.1 & $\mathrm{mg} / \mathrm{m}^{2}$ & lrl & $\begin{array}{l}\text { Arar and Collins, } \\
1997\end{array}$ & EPA 445.0 \\
\hline $\begin{array}{l}\text { Periphyton, bio- } \\
\text { mass, ash weight }\end{array}$ & 611 & 00572 & GRV15 & - & 0.1 & $\mathrm{~g} / \mathrm{m}^{2}$ & $\mathrm{mrl}$ & $\begin{array}{l}\text { Britton and Greeson, } \\
1987\end{array}$ & $\begin{array}{l}\text { USGS } \\
\text { Method } \\
\text { B-3520-85 }\end{array}$ \\
\hline $\begin{array}{l}\text { Periphyton, bio- } \\
\text { mass, dry weight }\end{array}$ & 603 & 00573 & GRV15 & - & 0.1 & $\mathrm{~g} / \mathrm{m}^{2}$ & $\mathrm{mrl}$ & $\begin{array}{l}\text { Britton and Greeson, } \\
1987\end{array}$ & $\begin{array}{l}\text { USGS } \\
\text { Method } \\
\text { B-3520-85 }\end{array}$ \\
\hline
\end{tabular}

${ }^{1}$ This report contains CAS Registry Numbers ${ }^{\circledR}$, which is a Registered Trademark of the American Chemical Society. CAS recommends the verification of the CAS Registry Numbers through CAS Client ServicesSM. 
Table 2.11. U.S. Geological Survey Organic Chemistry Research Laboratory Schedule for pesticides in sediment and biofilms.

[Method is described in Hladik and McWayne (2012). Abbreviations: NWIS, USGS National Water Information System; CAS, Chemical Abstracts Service1; g, gram; MDL, method detection level; $\mu \mathrm{g} / \mathrm{kg}$, microgram per kilogram; —, no data; na, not applicable]

\begin{tabular}{|c|c|c|c|c|}
\hline Compound & CAS number & $\begin{array}{l}\text { NWIS parameter code } \\
\text { for bed sediment }\end{array}$ & $\begin{array}{l}\text { MDL in sediment } \\
(\mu \mathrm{g} / \mathrm{kg})\end{array}$ & $\begin{array}{l}\text { MDL in biofilms } \\
(\mu \mathrm{g} / \mathrm{kg}) \text { for } 5 \mathrm{~g} \\
\text { sample }\end{array}$ \\
\hline $\begin{array}{l}\text { 2-Chloro-2,6-Diethylacetanilide } \\
\text { (CDEPA) }\end{array}$ & $6967-29-9$ & 68876 & 1.3 & 1.3 \\
\hline 3,4-Dichloroaniline & $95-76-1$ & 66585 & 1.3 & 1.3 \\
\hline 3,5-Dichloroaniline & $626-43-7$ & 67538 & 1.5 & 1.5 \\
\hline Allethrin & $584-79-2$ & 66588 & 1.7 & 1.7 \\
\hline Atrazine & $1912-24-9$ & 39631 & 1.5 & 1.5 \\
\hline Azinphos-methyl & $86-50-0$ & 64150 & 1.7 & 1.7 \\
\hline Azoxystrobin & $131860-33-8$ & 66591 & 0.9 & 0.9 \\
\hline Benfluralin (Benefin) & $1861-40-1$ & 68878 & 1.7 & 1.7 \\
\hline Butylate & $2008-41-5$ & 64152 & 1.3 & 1.3 \\
\hline Captan & $133-06-2$ & 68324 & 3.1 & na \\
\hline Carbaryl & $63-25-2$ & 64153 & 1.2 & 1.2 \\
\hline Carbofuran & $1563-66-2$ & 64154 & 1.2 & 1.2 \\
\hline Chlorothalonil & $1897-45-6$ & 62904 & 1.1 & na \\
\hline Chlorpyrifos & $2921-88-2$ & 81404 & 0.9 & 0.9 \\
\hline Clomazone & 81777-89-1 & 67564 & 2.0 & 2.0 \\
\hline Coumaphos & $56-72-4$ & 68882 & 1.2 & 1.2 \\
\hline Cycloate & $1134-23-2$ & 64155 & 0.8 & 0.8 \\
\hline DCPA (Dacthal) & $1861-32-1$ & 62905 & 1.7 & 1.7 \\
\hline Deltamethrin & $52918-63-5$ & 65110 & 1.3 & 1.3 \\
\hline Diazinon & $333-41-5$ & 39571 & 1.6 & 1.6 \\
\hline Difenoconazole & $119446-68-3$ & 67584 & 1.0 & na \\
\hline Dimethomorph & $110488-70-5$ & 68375 & 1.5 & na \\
\hline Dithiopyr & $97886-45-8$ & 68886 & 1.3 & 1.3 \\
\hline EPTC & $759-94-4$ & 64158 & 0.8 & 0.8 \\
\hline Esfenvalerate & $66230-04-4$ & 64159 & 1.0 & 1.0 \\
\hline Ethalfluralin & $55283-68-6$ & 64160 & 1.2 & 1.2 \\
\hline Etofenprox & 80844-07-1 & 67606 & 1.0 & 1.0 \\
\hline Famoxadone & $131807-57-3$ & 67611 & 1.7 & na \\
\hline Fenarimol & $60168-88-9$ & 67615 & 1.4 & na \\
\hline Fenbuconazole & $114369-43-6$ & 67620 & 1.8 & na \\
\hline Fenhexamide & $126833-17-8$ & 67624 & 2.5 & na \\
\hline Fenpropathrin & $39515-41-8$ & 65111 & 1.0 & 1.0 \\
\hline
\end{tabular}


Table 2.11. U.S. Geological Survey Organic Chemistry Research Laboratory Schedule for pesticides in sediment and biofilms.Continued

[Method is described in Hladik and McWayne (2012). Abbreviations: NWIS, USGS National Water Information System; CAS, Chemical Abstracts Service ${ }^{1}$; $\mathrm{g}$, gram; MDL, method detection level; $\mu \mathrm{g} / \mathrm{kg}$, microgram per kilogram; -, no data; na, not applicable]

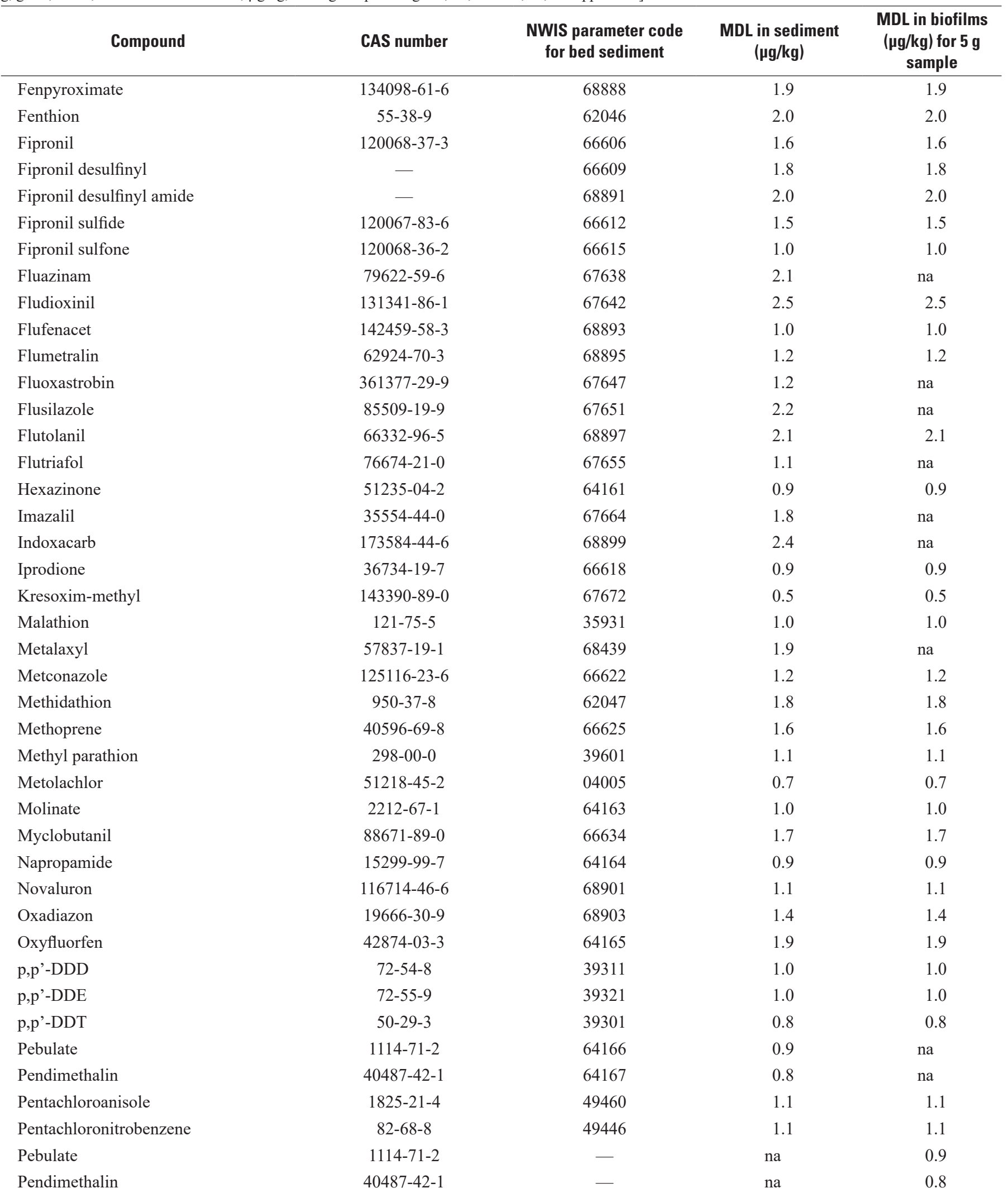


Table 2.11. U.S. Geological Survey Organic Chemistry Research Laboratory Schedule for pesticides in sediment and biofilms.Continued

[Method is described in Hladik and McWayne (2012). Abbreviations: NWIS, USGS National Water Information System; CAS, Chemical Abstracts Service'; g, gram; MDL, method detection level; $\mu \mathrm{g} / \mathrm{kg}$, microgram per kilogram; —, no data; na, not applicable]

\begin{tabular}{|c|c|c|c|c|}
\hline Compound & CAS number & $\begin{array}{l}\text { NWIS parameter code } \\
\text { for bed sediment }\end{array}$ & $\begin{array}{c}\text { MDL in sediment } \\
(\mu \mathrm{g} / \mathrm{kg})\end{array}$ & $\begin{array}{c}\text { MDL in biofilms } \\
\text { ( } \mu \mathrm{g} / \mathrm{kg} \text { ) for } 5 \mathrm{~g} \\
\text { sample }\end{array}$ \\
\hline Permethrin & $52645-53-1$ & 64168 & 0.9 & 0.9 \\
\hline Phenothrin & $26002-80-2$ & 65112 & 0.9 & 0.9 \\
\hline Phosmet & $732-11-6$ & 64169 & 0.9 & 0.9 \\
\hline Piperonyl butoxide & $51-03-6$ & 64170 & 1.2 & 1.2 \\
\hline Prometon & $1610-18-0$ & 82402 & 2.7 & 2.7 \\
\hline Prometryn & $7287-19-6$ & 78688 & 1.3 & 1.3 \\
\hline Propanil & $709-98-8$ & 66642 & 2.2 & 2.2 \\
\hline Propargite & $2312-35-8$ & 68907 & 2.2 & 2.2 \\
\hline Propiconazole & $60207-90-1$ & 66645 & 1.1 & 1.1 \\
\hline Pyrimethanil & $53112-28-0$ & 67719 & 1.1 & na \\
\hline Resmethrin & $10453-86-8$ & 65113 & 1.3 & 1.3 \\
\hline Simazine & $122-34-9$ & 39046 & 1.3 & 1.3 \\
\hline tau-fluvalinate & $69409-94-5$ & 65114 & 1.2 & 1.2 \\
\hline Tebuconazole & $107534-96-3$ & 66650 & 1.2 & na \\
\hline Tebupirimfos & - & 68911 & 1.5 & 1.5 \\
\hline Tebupirimfos oxon & $96182-53-5$ & 68913 & 2.0 & na \\
\hline Tefluthrin & $79538-32-2$ & 67733 & 0.7 & 0.7 \\
\hline Tetraconazole & $112281-77-3$ & 66656 & 1.1 & 1.1 \\
\hline Triallate & $2303-17-5$ & 68919 & 1.4 & 1.4 \\
\hline Tribuphos & $78-48-8$ & 39050 & 2.2 & 2.2 \\
\hline Trifloxystrobin & $141517-21-7$ & 66662 & 1.0 & 1.0 \\
\hline Triflumizole & 68694-11-1 & 67755 & 1.1 & na \\
\hline Trifluralin & $1582-09-8$ & 62902 & 0.9 & 0.9 \\
\hline Triticonazole & $131983-72-7$ & 67760 & 1.8 & na \\
\hline Vinclozolin & $50471-44-8$ & 67765 & 1.2 & 1.2 \\
\hline Zoxamide & $156052-68-5$ & 67770 & 1.1 & 1.1 \\
\hline
\end{tabular}

1This report contains CAS Registry Numbers ${ }^{\circledR}$, which is a Registered Trademark of the American Chemical Society. CAS recommends the verification of the CAS Registry Numbers through CAS Client ServicesSM. 
Table 2.12. U.S. Environmental Protection Agency Laboratory Schedule for pesticides in filtered water from small-volume pesticide autosamplers.

[EPA, U.S. Environmental Protection Agency; CAS, Chemical Abstracts Service ${ }^{1}$; ESI+, positive electrospray ionization mode; ESI-, negative electrospray ionization mode; MDL, method detection level; ng/L, nanograms per liter; - , no data]

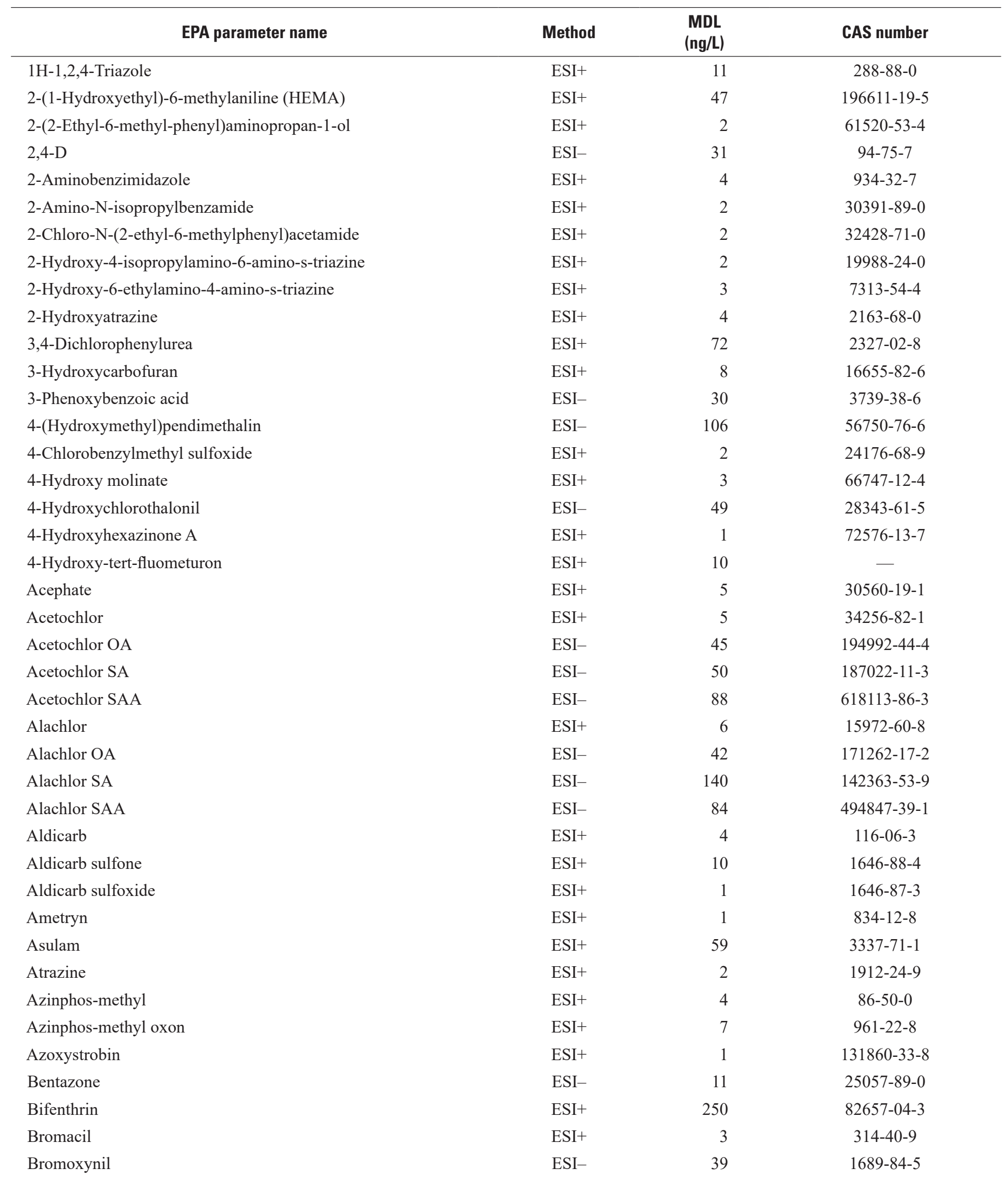


Table 2.12. U.S. Environmental Protection Agency Laboratory Schedule for pesticides in filtered water from small-volume pesticide autosamplers.-Continued

[EPA, U.S. Environmental Protection Agency; CAS, Chemical Abstracts Service1; ESI+, positive electrospray ionization mode; ESI-, negative electrospray ionization mode; MDL, method detection level; ng/L, nanograms per liter; —, no data]

\begin{tabular}{|c|c|c|c|}
\hline EPA parameter name & Method & $\begin{array}{c}\text { MDL } \\
\text { (ng/L) }\end{array}$ & CAS number \\
\hline Butralin & ESI+ & 4 & $33629-47-9$ \\
\hline Carbaryl & ESI+ & 3 & $63-25-2$ \\
\hline Carbendazim & ESI+ & 1 & $10605-21-7$ \\
\hline Chlorimuron-ethyl & ESI+ & 4 & $90982-32-4$ \\
\hline Chlorosulfonamide acid & ESI- & 37 & $130-45-0$ \\
\hline Chlorpyrifos & ESI+ & 25 & $2921-88-2$ \\
\hline Chlorpyrifos oxon & ESI+ & 2 & $5598-15-2$ \\
\hline Dacthal monoacid & ESI- & 250 & $887-54-7$ \\
\hline Dechlorofipronil & ESI- & 3 & - \\
\hline Dechlorometolachlor & ESI+ & 10,000 & $126605-22-9$ \\
\hline Deethylatrazine & ESI+ & 5 & $6190-65-4$ \\
\hline Deiodo flubendiamide & ESI+ & 4 & $1016160-78-3$ \\
\hline Deisopropyl prometryn & $\mathrm{ESI}+$ & 1 & $4147-57-3$ \\
\hline Deisopropylatrazine & ESI+ & 10 & $1007-28-9$ \\
\hline Demethyl hexazinone B & ESI+ & 1 & $56611-54-2$ \\
\hline Demethyl norflurazon & ESI+ & 2 & $23576-24-1$ \\
\hline Dichlorvos & ESI+ & 26 & $62-73-7$ \\
\hline Dicrotophos & ESI+ & 2 & $141-66-2$ \\
\hline Didealkylatrazine & ESI+ & 25 & $3397-62-4$ \\
\hline Diflubenzuron & ESI+ & 5 & $35367-38-5$ \\
\hline Diflufenzopyr & ESI- & 44 & 109293-97-2 \\
\hline Diketonitrile isoxaflutole & ESI- & 31 & $143701-75-1$ \\
\hline Dimethachlor SA & ESI- & 10 & \\
\hline Dimethenamid & ESI+ & 1 & $87674-68-8$ \\
\hline Dimethenamid OA & ESI- & 42 & $380412-59-9$ \\
\hline Dimethenamid SA & ESI- & 39 & $205939-58-8$ \\
\hline Dimethenamid SAA & ESI- & 94 & - \\
\hline Dimethoate & ESI+ & 1 & $60-51-5$ \\
\hline Dimethoate oxon & ESI+ & 1 & $1113-02-6$ \\
\hline Disulfoton & ESI+ & 12 & 298-04-4 \\
\hline
\end{tabular}


Table 2.12. U.S. Environmental Protection Agency Laboratory Schedule for pesticides in filtered water from small-volume pesticide autosamplers.-Continued

[EPA, U.S. Environmental Protection Agency; CAS, Chemical Abstracts Service1; ESI+, positive electrospray ionization mode; ESI-, negative electrospray ionization mode; MDL, method detection level; ng/L, nanograms per liter; - , no data]

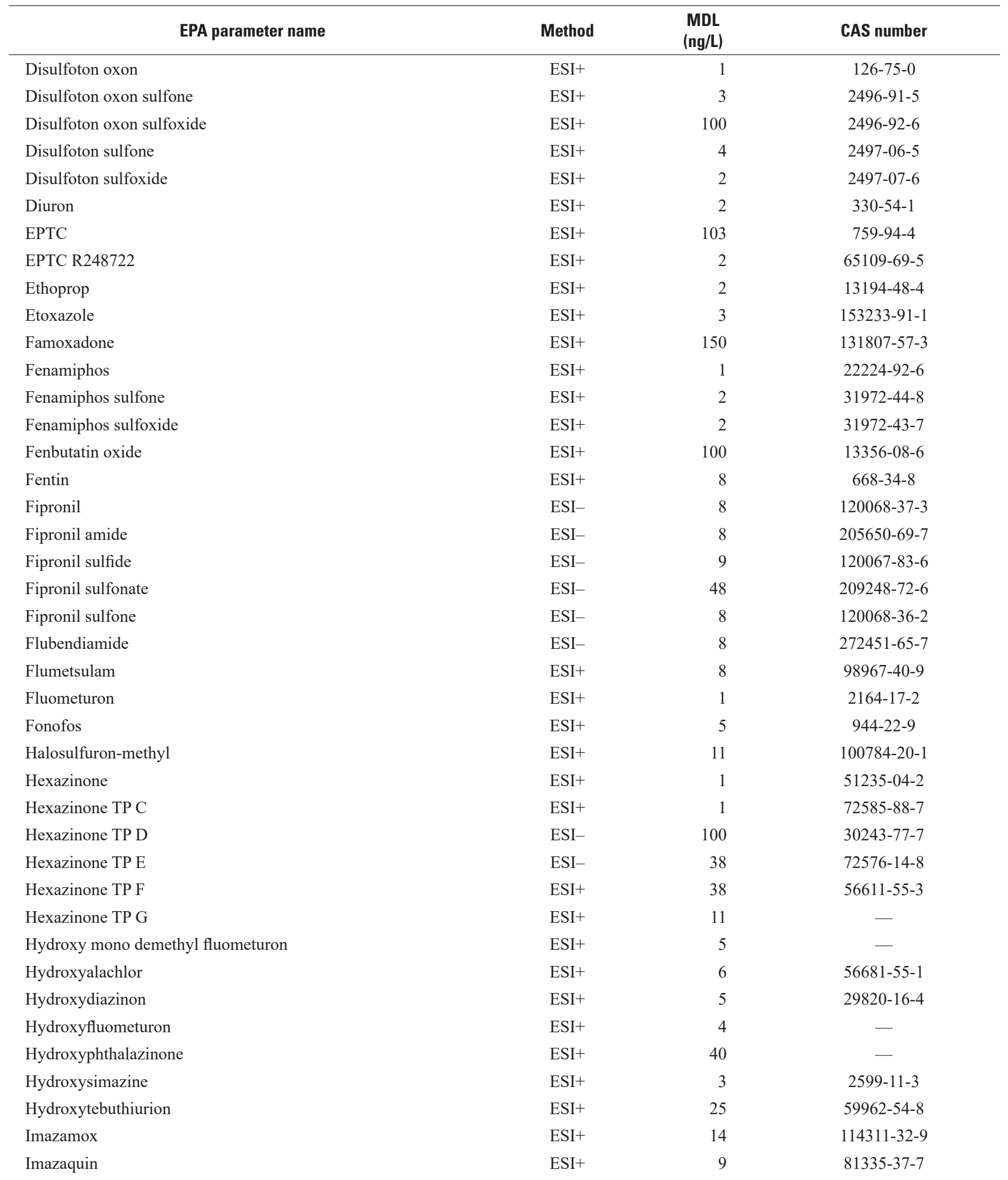


Table 2.12. U.S. Environmental Protection Agency Laboratory Schedule for pesticides in filtered water from small-volume pesticide autosamplers.-Continued

[EPA, U.S. Environmental Protection Agency; CAS, Chemical Abstracts Service1; ESI+, positive electrospray ionization mode; ESI-, negative electrospray ionization mode; MDL, method detection level; ng/L, nanograms per liter; —, no data]

\begin{tabular}{|c|c|c|c|}
\hline EPA parameter name & Method & $\begin{array}{c}\text { MDL } \\
\text { (ng/L) }\end{array}$ & CAS number \\
\hline Imazethapyr & ESI+ & 10 & $81335-77-5$ \\
\hline Indoxacarb & ESI+ & 12 & $173584-44-6$ \\
\hline Isoxaflutole & ESI+ & 15 & $141112-29-0$ \\
\hline Lactofen & ESI+ & 10 & $77501-63-4$ \\
\hline Linuron & ESI+ & 3 & $330-55-2$ \\
\hline Malaoxon & ESI+ & 1 & $1634-78-2$ \\
\hline Malathion & ESI+ & 2 & $121-75-5$ \\
\hline Methamidophos & ESI+ & 3 & $10265-92-6$ \\
\hline Methidathion & ESI+ & 5 & $950-37-8$ \\
\hline Methomyl & ESI+ & 2 & $16752-77-5$ \\
\hline Methomyl oxime & ESI+ & 250 & $13749-94-5$ \\
\hline Methoxyfenozide & ESI+ & 1 & $161050-58-4$ \\
\hline Metolachlor & ESI+ & 4 & $51218-45-2$ \\
\hline Metolachlor hydroxy morpholinone & ESI+ & 14 & $61520-54-5$ \\
\hline Metolachlor OA & ESI- & 74 & $152019-73-3$ \\
\hline Metolachlor SA & ESI- & 40 & 171118-09-5 \\
\hline Myclobutanil & ESI+ & 5 & $88671-89-0$ \\
\hline N-(3,4-Dichlorophenyl)-N-methylurea & ESI+ & 5 & $3567-62-2$ \\
\hline Naled & ESI+ & 15 & $300-76-5$ \\
\hline Nicosulfuron & ESI+ & 7 & 111991-09-4 \\
\hline Norflurazon & ESI+ & 3 & $27314-13-2$ \\
\hline Novaluron & ESI+ & 50 & $116714-46-6$ \\
\hline O-Ethyl-O-methyl-S-propyl phosphorothioate & ESI+ & 2 & $76960-87-7$ \\
\hline Orthosulfamuron & ESI+ & 3 & $213464-77-8$ \\
\hline Oryzalin & ESI- & 5 & $19044-88-3$ \\
\hline Oxamyl & ESI+ & 1 & $23135-22-0$ \\
\hline Oxamyl oxime & ESI+ & 3 & $30558-43-1$ \\
\hline Oxyfluorfen & ESI+ & 500 & $42874-03-3$ \\
\hline Paraoxon & ESI+ & 2 & $311-45-5$ \\
\hline Paraoxon-methyl & ESI+ & 12 & $950-35-6$ \\
\hline
\end{tabular}


Table 2.12. U.S. Environmental Protection Agency Laboratory Schedule for pesticides in filtered water from small-volume pesticide autosamplers.-Continued

[EPA, U.S. Environmental Protection Agency; CAS, Chemical Abstracts Service1; ESI+, positive electrospray ionization mode; ESI-, negative electrospray ionization mode; MDL, method detection level; ng/L, nanograms per liter; - , no data]

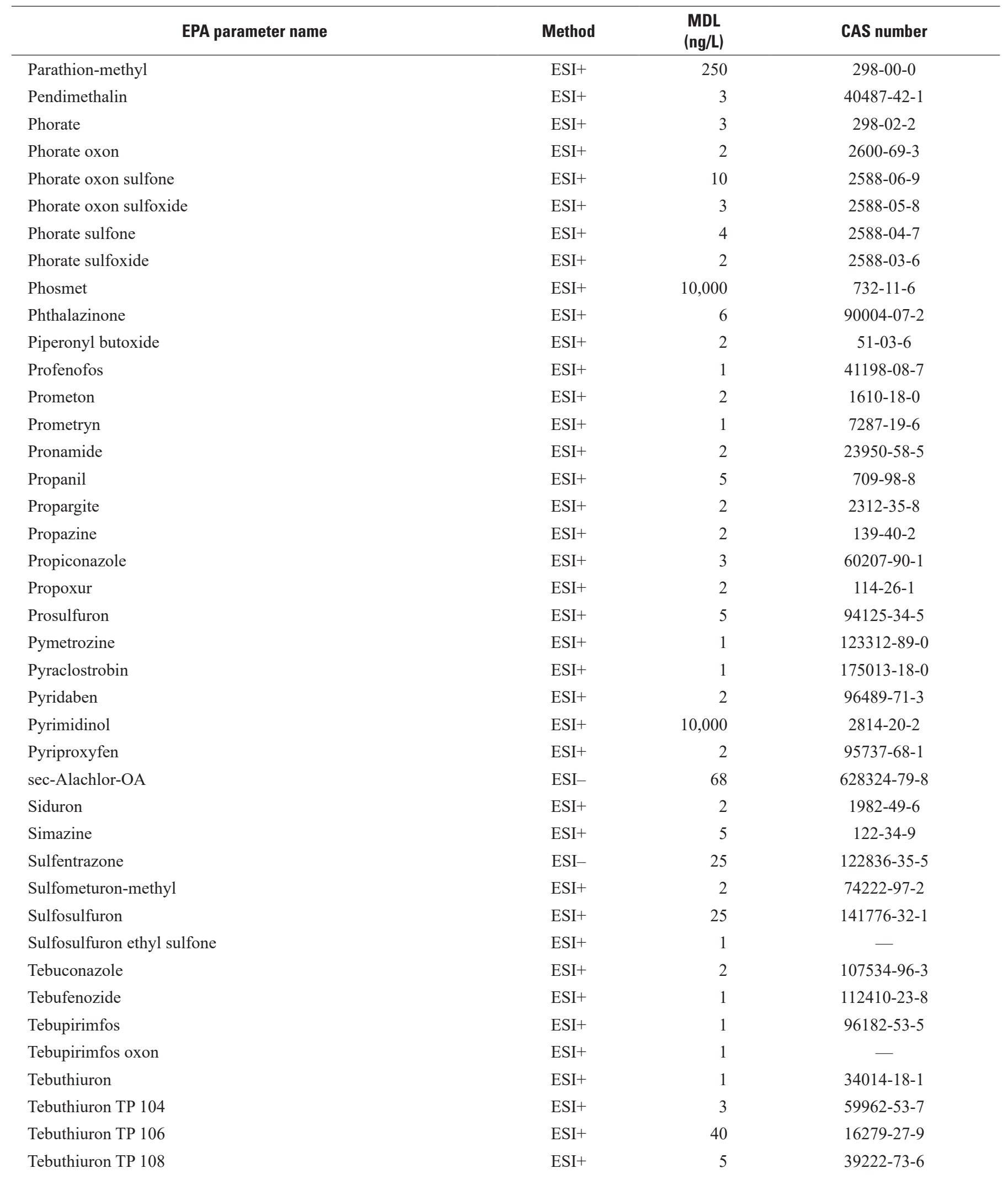


Table 2.12. U.S. Environmental Protection Agency Laboratory Schedule for pesticides in filtered water from small-volume pesticide autosamplers.-Continued

[EPA, U.S. Environmental Protection Agency; CAS, Chemical Abstracts Service1; ESI+, positive electrospray ionization mode; ESI-, negative electrospray ionization mode; MDL, method detection level; ng/L, nanograms per liter; - , no data]

\begin{tabular}{lccc}
\hline \multicolumn{1}{c}{ EPA parameter name } & Method & $\begin{array}{c}\text { MDL } \\
\text { (ng/L) }\end{array}$ & CAS number \\
\hline Tebuthiuron TP 109 (OH) & ESI+ & 13 & $139888-73-6$ \\
Terbacil & ESI+ & 15 & $5902-51-2$ \\
Terbufos & ESI + & 4 & $13071-79-9$ \\
Terbufos oxon & ESI + & 2 & $56070-14-5$ \\
Terbufos oxon sulfone & ESI+ & 5 & $56070-15-6$ \\
Terbufos oxon sulfoxide & ESI+ & 2 & $56165-57-2$ \\
Terbufos sulfone & ESI+ & 5 & $56070-16-7$ \\
Terbufos sulfoxide & ESI+ & 1 & $10548-10-4$ \\
Terbuthylazine & ESI+ & 1 & $5915-41-3$ \\
Tetraconazole & ESI+ & 5 & $112281-77-3$ \\
Thiobencarb & ESI+ & 2 & $28249-77-6$ \\
trans-Permethrin & ESI+ & 100 & $61949-77-7$ \\
Triallate & ESI+ & 10 & $2303-17-5$ \\
Triallate SA & ESI- & 27 & $65600-62-6$ \\
Tribuphos & ESI+ & 5 & $78-48-8$ \\
Triclopyr & ESI- & 44 & $55335-06-3$ \\
Trifloxystrobin & ESI+ & 1 & $141517-21-7$ \\
\hline
\end{tabular}

${ }^{1}$ This report contains CAS Registry Numbers ${ }^{\circledR}$, which is a Registered Trademark of the American Chemical Society. CAS recommends the verification of the CAS Registry Numbers through CAS Client ServicesSM.

\section{References Cited}

Arar, E.J., and Collins, G.B., 1997, Method 445.0-In vitro determination of chlorophyll $a$ and pheophytin $a$ in marine and freshwater algae by fluorescence (rev. 1.2, September 1997): Cincinnati, Ohio, U.S. Environmental Protection Agency, National Exposure Research Laboratory, Office of Research and Development, 22 p., https://cfpub.epa.gov/si/si_public_record_report. $\mathrm{cfm} ? \mathrm{Lab}=$ NERL \&dirEntryId=309417.

Britton, L.J., and Greeson, P.E., eds., 1989, Gravimetric method for biomass, in Methods for collection and analysis of aquatic biological and microbiological samples: U.S. Geological Survey Techniques of Water-Resources Investigations, book 5, chap. A4, p. 139-140, https://doi.org/10.3133/twri05A4.

Burkhardt, M.R., Zaugg, S.D., Smith, S.G., and ReVello, R.C., 2006, Determination of wastewater compounds in sediment and soil by pressurized solvent extraction, solid-phase extraction, and capillary-column gas chromatography/mass spectrometry: U.S. Geological Survey Techniques and Methods, book 5, chap. B2, 34 p., https://doi.org/10.3133/tm5B2.
Fishman, M.J., ed., 1993, Methods of analysis by the U.S. Geological Survey National Water Quality Laboratory-Determination of inorganic and organic constituents in water and fluvial sediments, U.S. Geological Survey Open-File Report 93-125, 217 p., https://doi.org/10.3133/ofr93125.

Fishman, M.J., and Friedman, L.C., 1989, Methods for determination of inorganic substances in water and fluvial sediments: U.S. Geological Survey Techniques of Water-Resources Investigations, book 5, chap. A1, 545 p., https://doi.org/10.3133/twri05A1.

Foreman, W.T., Gray, J.L., ReVello, R.C., Lindley, C.E., Losche, S.A., and Barber, L.B., 2012, Determination of steroid hormones and related compounds in filtered and unfiltered water by solid-phase extraction, derivatization, and gas chromatography with tandem mass spectrometry: U.S. Geological Survey Techniques and Methods, book 5, chap. B9, 118 p., https://doi.org/10.3133/tm5B9. 
Furlong, E.T., Noriega, M.C., Kanagy, C.J., Kanagy, L.K., Coffey, L.J., and Burkhardt, M.R., 2014, Determination of human-use pharmaceuticals in filtered water by direct aqueous injection-high-performance liquid chromatography/tandem mass spectrometry: U.S. Geological Survey Techniques and Methods, book 5, chap. B10, 49 p., https://doi.org/10.3133/tm5B10.

Gellis, A.C., Fuller, C.C., and Van Metre, P., 2017, Sources and ages of fine-grained sediment to streams using fallout radionuclides in the midwestern United States: Journal of Environmental Management, v. 194, p. 73-85, https://doi.org/10.1016/j.jenvman.2016.06.018.

Hladik, M.L., and McWayne, M.M., 2012, Methods of analysis - Determination of pesticides in sediment using gas chromatography/mass spectrometry: U.S. Geological Survey Techniques and Methods, book 5, chap. C3, 18 p., https://doi.org/10.3133/tm5C3.

Mahler, B.J., Van Metre, P.C., Wilson, J.T., Musgrove, M., Zaugg, S.D., and Burkhardt, M.R., 2009, Fipronil and its degradates in indoor and outdoor dust: Environmental Science \& Technology, v. 43, no. 15, p. 5665-5670, https://doi.org/10.1021/es901292a.

O’Dell, J.W., 1993, Method 365.1, Revision 2.0Determination of phosphorus by semi-automated colorimetry: Cincinnati, Ohio, U.S. Environmental Protection Agency, Office of Research and Development, 17 p., https://www.epa.gov/sites/production/files/2015-08/ documents/method_365-1_1993.pdf.

Patton, C.J., and Kryskalla, J.R., 2003, Methods of analysis by the U.S. Geological Survey National Water Quality Laboratory_Evaluation of alkaline persulfate digestion as an alternative to Kjeldahl digestion for determination of total and dissolved nitrogen and phosphorus in water: U.S. Geological Survey Water-Resources Investigations Report 2003-4174, 33 p., https://doi.org/10.3133/wri034174.
Patton, C.J., and Kryskalla, J.R., 2011, Colorimetric determination of nitrate plus nitrite in water by enzymatic reduction, automated discrete analyzer methods: U.S. Geological Survey Techniques and Methods, book 5, chap. B8, 34 p., https://doi.org/10.3133/tm5B8.

Sandstrom, M.W., Kanagy, L.K., Anderson, C.A., and Kanagy, C.J., 2016, Determination of pesticides and pesticide degradates in filtered water by direct aqueous-injection liquid chromatography-tandem mass spectrometry: U.S. Geological Survey Techniques and Methods, book 5, chap B11, 54 p., accessed December 8, 2015, at https://doi.org/10.3133/tm5B11.

U.S. Environmental Protection Agency, 2014, Method 8270D — Semivolatile organic compounds by gas chromatography/mass spectrometry: U.S. Environmental Protection Agency, SW-846 Update V, 71 p., https://19january2017snapshot.epa.gov/sites/production/ files/2015-12/documents/8270d.pdf.

Wagner, R.J., Moran, P.W., Zaugg, S.D., Sevigny, J.M., and Pope, J.M., 2014, Contaminants of emerging concern in the lower Stillaguamish River Basin, Washington, 2008-11 (ver. 2.0, June 2016): U.S. Geological Survey Open-File Report 2014-1028, 14 p., accessed August 1, 2016, at https://doi.org/10.3133/ofr20141028.

Zaugg, S.D., Smith, S.G., and Schroeder, M.P., 2006, Determination of wastewater compounds in whole water by continuous liquid-liquid extraction and capillary-column gas chromatography/mass spectrometry: U.S. Geological Survey Techniques and Methods, book 5, chap. B4, 30 p., https://doi.org/10.3133/tm5B4. 
For more information concerning the research in this report, contact the Director, California Water Science Center

U.S. Geological Survey

6000 J Street, Placer Hall

Sacramento, California 95819

https://ca.water.usgs.gov

Publishing support provided by the U.S. Geological Survey Science Publishing Network, Sacramento Publishing Service Center 

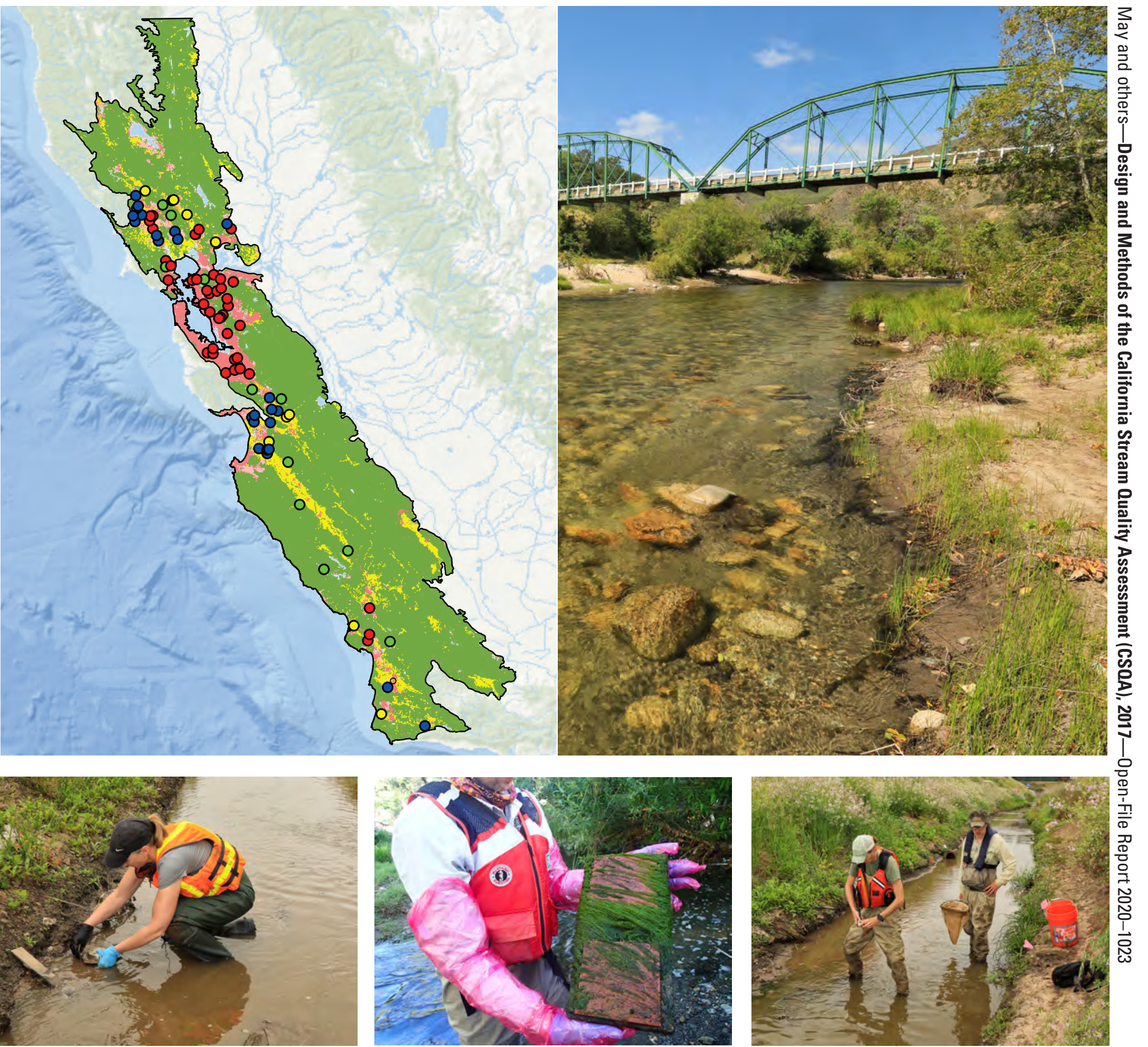